\title{
Entwicklung eines quantitativen Verfahrens zur Streufeld- und Magnetisierungsbestimmung magnetischer Strukturen
}

\author{
Dissertation \\ zur Erlangung des Doktorgrades \\ der Mathematisch-Naturwissenschaftlichen Fakultäten \\ der Georg-August-Universität zu Göttingen
}

vorgelegt von

Sebastian Dreyer

aus Hannover

Göttingen, Mai 2007 
Referent:

Korreferent:

Tag der mündlichen Prüfung:
Priv.Doz. Dr. Christian Jooß

Prof. Dr. Markus Münzenberg

03.07.2007 
Für Stephanie 



\section{Inhaltsverzeichnis}

1. Einleitung 1

2. Theoretische Grundlagen 5

2.1. Grundlagen der Magnetostatik . . . . . . . . . . . . . . . 5

2.2. Ansatz zur Inversion von Streufeldmessungen 2-dim. Strukturen . . . . . . 9

3. Experimentelle Methoden 13

3.1. Herstellung hartmagnetischer Filme . . . . . . . . . . . . . . . . . 13

3.2. Probenstrukturierung mittels Elektronenstrahllithographie (EBL) . . . . . 14

3.3. Röntgendiffraktometrie (XRD) . . . . . . . . . . . . . . . . 15

3.4. Rasterelektronenmikroskopie (REM) . . . . . . . . . . . . . . 16

3.5. Raster- und magnetische Kraftmikroskopie . . . . . . . . . . . . . . . . . . 17

3.6. SQUID-Magnetometrie . . . . . . . . . . . . . . . . 18

4. Charakterisierung hartmagnetischer Filme und Strukturen 21

4.1. Eigenschaften und Wachstum von FePt-Filmen . . . . . . . . . . . . . . . 21

4.2. Eigenschaften und Wachstum von CoPt-Filmen . . . . . . . . . . . . . 24

4.3. Magnetische Charakterisierung der FePt- und CoPt-Filme . . . . . . . . 26

4.4. Wachstum und Eigenschaften von $\mathrm{PrCo}_{5}$-Filmen . . . . . . . . . . . . . 29

4.5. Strukturierung hartmagnetischer Schichten . . . . . . . . . . . . . . . 31

4.6. Hartmagnetische Strukturen als Modellsysteme . . . . . . . . . . . . . . 32

5. Magneto-optische Messmethode zur lokalen magnetischen Abbildung 35

5.1. Messprinzip zur Abbildung von Streufeldern magnetischer Strukturen . . . 35

5.2. Experimenteller Messaufbau . . . . . . . . . . . . . . . . . . . 39

5.3. Verbesserung des magnetischen Kontrastes mittels Differenzbildtechnik . . 42

5.4. Optimierung des magnetischen Auflösungsvermögens . . . . . . . . . . . . 47

5.5. Simulation von Streufeldern bei rein senkrechter Magnetisierung . . . . . . 50

5.6. Simulation von Streufeldern bei reiner inplane Magnetisierung . . . . . . . 55

6. Rekonstruktion von Magnetisierungsverteilungen aus Streufeldmessungen $\mathbf{5 7}$

6.1. Rekonstruktion senkrechter Magnetisierungsverteilungen . . . . . . . . . . 57

6.2. Rekonstruktion in der Ebene ausgerichteter Magnetisierungsverteilungen . 72

7. Anfangsstadien der Ummagnetisierung hartmagnetischer CoPt-Strukturen 81

7.1. Ummagnetisierung uniaxialer Hartmagnete . . . . . . . . . . . . . . . . . 82

7.2. Experimentelle Ergebnisse zum Ummagnetisierungsverhalten von CoPt-

Strukturen unterschiedlicher Größe und Form . . . . . . . . . . . . . 83

7.3. Diskussion des Ummagnetisierungsverhaltens der CoPt-Strukturen . . . . . 91 
8. Ansatz zur Kalibrierung eines magnetischen Kraftmikroskops mittels quantitativer MOIF-Messungen

$\begin{array}{ll}\text { 9. Zusammenfassung } & 105\end{array}$

$\begin{array}{ll}\text { A. Mathematischer Anhang } & 109\end{array}$

Literaturverzeichnis 


\section{Einleitung}

Die Charakterisierung magnetischer Nanostrukturen stellt heutzutage ein Gebiet intensiver Forschung dar. Aufgrund der enormen technologischen Relevanz nanoskaliger magnetischer Strukturen, wie sie z.B. in nicht-flüchtigen Speichermedien (Patterned Media $[1,2,3,4,5]$ und $M R A M[6,7])$ und hochintegrierten, magnetoelektronischen Bauelementen $[8,9]$ zum Einsatz kommen, ist auch der Bedarf an magnetischen Abbildungsmethoden gestiegen [10, 11]. Zur Analyse ausgedehnter, zweidimensionaler Gitter magnetischer Elemente (Datenspeicher) gibt es eine besondere Notwendigkeit der Kombination von Methoden mit einem großen Messausschnitt und hoher Ortsauflösung. Da sich theoretische Vorhersagen auf Basis mikromagnetischer Rechnungen in der Beschreibung realer Materialien häufig als zu komplex oder mangels vertrauenswürdiger Startparameter als wenig aussagekräftig erweisen, gibt es neben dem Ziel einer ständigen Verbesserung der experimentellen Auflösung, ein starkes Interesse an quantitativen magnetischen Messverfahren.

Der Reduktion der Strukturdimensionen in modernen elektronischen Bauteilen und deren Einfluss auf das magnetische Verhalten einzelner Strukturen, bzw. des ganzen Kollektivs, kommt eine Schlüsselrolle zu. Eine zentrale Größe für das Verständis dieses Verhaltens sind die verschiedenen Anisotropieenergien, die sich aus Volumen- (magnetokristalline Anisotropie) und Oberflächenbeiträgen (Formanisotropie Oberflächenbeiträge zur Kristallanisotropie) zusammensetzen. Mit Reduktion der Elementgrösse vergrößert sich das Verhältnis aus Oberfläche und Volumen, so dass die durch Geometrie und Oberflächen induzierten Beiträge an Bedeutung zunehmen [12].

In Anordnungen von magnetischen Elementen sind aber nicht nur die Strukturdimensionen alleine für das magnetische Verhalten verantwortlich, sondern es kann mit sinkendem Strukturabstand zu einer zusätzlichen Konfigurationsanisotropie kommen. Der Grund dafür liegt in einer magnetostatischen Wechselwirkung benachbarter Strukturen über ihre Streufelder. Gerade in künstlich erzeugten, regelmäßigen Anordnungen ist es möglich, einen Übergang von einem individuellen Verhalten einzelner Elemente zu einem kollektiven des gesamten Ensembles zu induzieren [13, 14, 15, 16, 17, 18]. Bei der Datenspeicherung in einzelnen Elementen ist es beispielsweise wichtig, dass es nicht zu einer gegenseitigen Beeinflussung kommen darf. Die Rolle der magnetostatischen Wechselwirkung in Konkurrenz bzw. im Zusammenwirken mit anderen Wechselwirkungen ist bis heute noch wenig verstanden. In solchen Systemen kann die Quantifizierung der Wechselwirkungspotentiale durch Bestimmung der Magnetisierung bzw. der Streufelder der Strukturen einen wesentlichen Beitrag zum Verständnis des kollektiven Verhaltens liefern.

Prinzipiell bietet die quantitative Messung von Streufeldern einen Zugang zur Berechnung von Magnetisierungsverteilungen von Strukturen mittels inverser mathematischer Formalismen. Da das Streufeld einer Probe von dem gesamten magnetischen Volumen erzeugt 
wird, ist eine entsprechende Messung nicht nur von Oberflächenbeiträgen abhängig, wie z.B. bei Standardverfahren wie Kerr- oder Elektronenmikroskopie mit Polarisationsanalyse, die stark von der Morphologie und damit auch vom Strukturierungsprozess selber beeinflusst werden können. Die Kenntnis der lokalen Magnetisierungsverteilung erlaubt desweiteren die Berechnung vieler magnetischer Kenngrössen eines Systems, so dass deren quantitative Bestimmung die fundamentale Grundlage eines Verständnisses der verschiedensten Wechselwirkungsbeiträge und magnetischen Charakteristika darstellt.

Die in dieser Arbeit verwendete Magneto-Optische Indikatorfilm-Methode (MOIF) ist in der Lage genau solche quantitativen Informationen auf einer Längenskala von $1 \mathrm{~cm}$ bis zu $1 \mu \mathrm{m}$ zu liefern. Basierend auf dem ausgeprägten magneto-optischen FaradayEffekt (MOFE) in speziellen Sensorschichten, zeigt sich die MOIF sensibel auf die normal zur Messebene stehende Komponente des Streufeldes der zu untersuchenden Strukturen [19, 20]. Das Messsignal kann einfach durch externe Felder kalibriert werden, so dass ein Zugang zur quantitativen Bestimmung der entsprechenden Feldverteilung besteht. Die Vorteile liegen in der Verwendbarkeit bei externen magnetischen Feldern in einem breiten Temperatur- und Druckbereich (von UHV bis normalem Luftdruck), sowie der zerstörungsfreien Messung beliebiger Probenmaterialien bei geringem Präparationsaufwand. Während die MOIF-Methode im Bereich der Untersuchung von Supraleitern inzwischen eine Standardcharakterisierungsmethode darstellt (siehe z.B. [21, 22, 23, 24, 25]), wird sie für die Analyse ferromagnetischer Strukturen eher selten eingesetzt [26, 27, 28, 29, 30, 31], da die technisch interessanten Strukturgrößen und die damit verbundenen Streufelder im Vergleich zu den Supraleitern um bis zu einer Größenordnung kleiner sein können. In der Regel wird dafür der bekanntere, dem MOFE verwandte, magneto-optische Kerr-Effekt (MOKE) verwendet [32, 33, 34], welcher die Oberflächenmagnetisierung eines Ferromagneten direkt abbildet. Im Gegensatz zur MOIF-Methode ist die Kerrmikroskopie jedoch quantitativ nur schwer durchführbar und erfordert sehr hohe Oberflächenqualitäten der Proben [35, 36]. Die Herausforderung in der Anwendung der MOIF-Methode für quantitative Streufelduntersuchungen liegt wiederum in der Optimierung bezüglich des Auflösungsvermögens und der Sensitivität. In dieser Arbeit kommt den beiden genannten Faktoren deshalb eine grundlegende Bedeutung zu, die in Kapitel 5 beschrieben werden.

Die Berechnung der lokalen Magnetisierungsverteilung von Strukturen nach Messung des Streufeldes erfordert desweiteren die Entwicklung fortgeschrittener inverser mathematischer Methoden. Die Basis dieser bilden sogenannte Transferfunktionen, die einen sehr allgemeingültigen und umfassenden Zugang zur Rekonstruktion der Magnetisierung gewährleisten, da sie die Möglichkeit der Berücksichtigung sämtlicher messspezifischer Limitierungen, neben der prinzipiellen Abhängigkeit der Streufeldverteilung von den Probendimensionen, bieten [37, 38]. Die Herleitung einer sogenannten Abbildungstransferfunktion für die MOIF-Methode, die sowohl die begrenzte optische Auflösung als auch die endliche Sensordicke bei der Magnetisierungsbestimmung mitberücksichtigt, stellt einen wesentlichen Beitrag dieser Arbeit dar. Die theoretischen Grundlagen dazu werden in Kapitel 2 hergeleitet und beschrieben.

Die Herstellung und magnetische Abbildung von Modellsystemen legt im Weiteren den Grundstein der Entwicklung der quantitativen Rekonstruktion von Magnetisierungsverteilungen auf Basis der Streufeldmessungen durch der MOIF-Methode. Das Ziel komplexere Verteilungen quantitativ erfassen zu können, setzt die Möglichkeit einer Charakterisierung 
zunächst einfacher, definierter Magnetisierungszustände voraus, indem an wohldefinierten Strukturen die Etablierung der Beziehung von $\vec{M}$ und $\vec{H}$ vollzogen werden kann. Die magnetischen Konfigurationen solcher Testproben sollten vor allem durch die entsprechenden Abbildungsmethoden nur minimal beeinflusst werden und große, wohl definierte Streufelder erzeugen. Die Auswahl der verwendeten Materialsysteme in dieser Arbeit gliedert sich nach der Möglichkeit die Magnetisierung entlang ausgezeichneter kristallographischer Orientierungen ausrichten zu können. Die Systeme FePt und CoPt dienen dabei als Testsysteme für rein senkrechte Magnetisierungsverteilungen und PrCo für rein in der Ebene ausgezeichnete. Durch die hohe magnetokristalline Anisotropie der ausgewählten Materialkombinationen können remanente, homogen aufmagnetisierte Strukturen definierter Größe und Form präpariert werden [39, 40, 41, 42, 43, 44], die ideal zur Kalibrierung geeignet sind, da sie alle oben erwähnten Anforderungen erfüllen. Eine gute strukturelle und magnetische Charakterisierung ist dabei essentiell, deren Ergebnisse in Kapitel 4 dargestellt sind.

Um einerseits die Sensorkalibrierung zu verifizieren als auch die Aussagekraft von theoretisch berechneten Feldverteilungen zu untermauern, werden in der Arbeit die experimentell bestimmten Streufelder der Strukturen mit Simulationen verglichen. Die Berechnungen dienen später vor allem dazu, die Grenzen der Magnetisierungsrekonstruktion mittels MOIF zu quantifizieren. Der eindomänige Zustand der Eichproben erlaubt dabei die magnetostatische Berechnung der $H_{z}$-Verteilungen, wodurch die Verwendung mikromagnetischer Simulationen vermieden werden kann.

Eine entscheidende Stärke der MOIF-Methode liegt in der Möglichkeit große Anordnungen von Strukturen unterschiedlicher Form und Größe in einer Aufnahme quantitativ analysieren zu können. Dies erlaubt die Untersuchung von Mechanismen bei der Ummagnetisierung solcher Anordnungen. Im Vordergrund von Kapitel 7 steht deshalb die Analyse des größen- und formabhängigen Ummagnetisierungsprozesses von hartmagnetischen Elemente in solchen Anordnungen. Die Verteilung lokaler Inhomogenitäten, die das Ummagnetisierungsverhalten der magnetischen Elemente in Abhängigkeit ihrer Größe wesentlich beeinflussen, ist von entscheidender Bedeutung. Die Charakterisierung der magnetischen Substruktur jenseits der Auflösungsgrenze der Magneto-Optik erfolgt durch magnetische Kraftmikroskopiemessungen (MFM). Es zeigt sich, dass die Verbindung beider Methoden dazu genutzt werden kann, ein große Längenskalen umfassendes quantitatives Bild der Magnetisierungszustände und Streufeldverteilungen hartmagnetischer Elemente zu geben $[45,46]$.

Im letzten Kapitel dieser Arbeit wird die quantitative Ankopplung der beiden streufeldsensitiven Methoden im Sinne einer Kalibrierung eines magnetischen Kraftmikroskopes beschrieben. Ziel ist die Untersuchung einer möglichen Eichung anhand von FePt Eichstandards unter Verwendung der quantitativen Streufeldinformationen aus den MOIFMessungen bzw. der rekonstruierten Magnetisierungsverteilungen. 


\section{Theoretische Grundlagen}

In diesem Kapitel sollen kurz die theoretischen Grundlagen vorgestellt werden, auf denen die Rekonstruktion einer Magnetisierungsverteilung aus Streufeldmessungen basiert. Über dieses Kapitel hinausgehende Details zur Magnetostatik sind in verschiedenen Lehrbüchern nachlesbar (z.B. [47, 48]).

\subsection{Grundlagen der Magnetostatik}

Ausgangspunkt für die folgenden Betrachtungen sind die beiden grundlegenden Differentialgleichungen der Magnetostatik in dichter Materie (makroskopische Maxwellgleichungen)

$$
\begin{aligned}
\vec{\nabla} \cdot \vec{B}(\vec{r}) & =0 \\
\vec{\nabla} \times \vec{H}(\vec{r}) & =\vec{\jmath}(\vec{r}) .
\end{aligned}
$$

Das magnetische Feld $\vec{H}(\vec{r})$ ergibt sich dabei aus der sogenannten Materialgleichung

$$
\vec{H}(\vec{r})=\frac{1}{\mu_{0}} \vec{B}(\vec{r})-\vec{M}(\vec{r})
$$

und berücksichtigt im Mittel die Beiträge der atomaren Ströme in Form einer mittleren magnetischen Momentdichte bzw. makroskopischen Magnetisierung $\vec{M}(\vec{r})$. Im Folgenden wird die Stromdichte freier Ladungsträger $\vec{\jmath}(\vec{r})$ gleich Null gesetzt $(\vec{\nabla} \times \vec{H}(\vec{r})=0)$, d.h. es liegen keine elektrischen Felder an. Die experimentell untersuchten Materialien, die in Kapitel 4 vorgestellt werden, sind aufgrund ihrer hohen Anisotropien ideale Kandidaten für Permanentmagnete. Deren Magnetisierung kann bei nicht zu großen Feldern näherungsweise als unabhängig vom äußeren Feld betrachtet werden, so dass man $\vec{M}(\vec{H})$ als rein ortsabhhängig $\vec{M}(\vec{r})$ annehmen kann. Diese Voraussetzung resultiert nun in zwei komplentären Beschreibungsweisen, welche sich aus den Lösungen der beiden obigen Differentialgleichungen ergeben.

Die Quellenfreiheit der magnetischen Flussdichte gemäss Gleichung 2.1 erlaubt die Berechnung von $\vec{B}(\vec{r})$ aus der Rotation eines Vektorfeldes, dem sogenannten magnetischen Vektorpotential $\vec{A}_{M}(\vec{r})$, gemäß

$$
\vec{B}(\vec{r})=\vec{\nabla} \times \vec{A}_{M}(\vec{r})
$$

Aus den Gl. 2.2 und 2.3 folgt für die einzelnen Komponenten des Vektorpotentials ( $\mathrm{i}=\mathrm{x}$, y, z) unmittelbar eine der Poissongleichung äquivalente Darstellung 


$$
\Delta A_{M, i}(\vec{r})=-\mu_{0} \jmath_{M, i}(\vec{r})
$$

mit der effektiven Magnetisierungsstromdichte $\vec{\jmath}_{M}(\vec{r})=\vec{\nabla} \times \vec{M}(\vec{r})$, die den solenoiden Anteil der Magnetisierung repräsentiert. In dieser Form liegt der Gleichung eine Eichtransformation des Vektorpotentials zugrunde, da dieses nur von der Rotation, also dem Wirbelanteil der magnetischen Flussdichte, festgelegt wird. Da statische Ströme stets quellenfrei sind (Erhaltungssatz), ist eine Eichung des Vektorpotentials auf jeden Fall möglich.

Nach Lösen der Poissongleichung 2.5 mit Hife des Greenschen Formalismus (detaillierte Darstellung siehe z.B. [49]) folgt im unendlichen Raum, d.h. ohne Randbedingungen im Endlichen, für das Vektorpotential

$$
\vec{A}(\vec{r})=\frac{\mu_{0}}{4 \pi} \int_{-\infty}^{\infty} \frac{\vec{\jmath}_{M}\left(\vec{r}^{\prime}\right)}{\left|\vec{r}-\vec{r}^{\prime}\right|} d^{3} r^{\prime}
$$

Damit ergibt sich die magnetische Flussdichte zu

$$
\vec{B}(\vec{r})=\frac{\mu_{0}}{4 \pi} \int_{-\infty}^{\infty} \vec{\jmath}_{M}\left(\vec{r}^{\prime}\right) \times \frac{\vec{r}-\vec{r}^{\prime}}{\left|\vec{r}-\vec{r}^{\prime}\right|^{3}} d^{3} r^{\prime}
$$

Mit der Greenschen Funktion $G\left(\vec{r}, \vec{r}^{\prime}\right)=\left|\vec{r}-\vec{r}^{\prime}\right|^{-1}$, die den asymptotischen Abfall der Flussdichte auf Null im Unendlichen gewährleistet, ist die Lösung eindeutig. Gleichung 2.7, in ähnlicher Form auch als Biot-Savart'sches Gesetz bekannt, wird in den Kapiteln 5.5 und 5.6 für die Vorwärtsrechnungen der magnetischen Streufelder von kleinen magnetischen Strukturen verwendet.

Die Wirbelfreiheit des magnetischen Feldes bei Verschwinden der makroskopischen Stromdichte impliziert direkt die Darstellung von $\vec{H}$ durch ein skalares, magnetisches Potential

$$
\vec{H}(\vec{r})=-\vec{\nabla} \phi_{M}(\vec{r})
$$

das wiederum auf eine Poissongleichung

$$
\Delta \phi_{M}(\vec{r})=-\rho_{M}(\vec{r})
$$

und die Einführung einer effektiven magnetischen Volumenladungsdichteverteilung gemäß $\rho_{M}(\vec{r})=\vec{\nabla} \cdot \vec{M}(\vec{r})$ führt. $\rho_{M}$ repräsentiert in diesem Fall den Potentialanteil der Magnetisierung. Gleichung 2.9 stellt das Äquivalent zu der aus der Elektrostatik bekannten Poissongleichung für den magnetostatischen Fall dar. Die magnetische Ladungsdichteverteilung ist als eine effektive Größe zu verstehen, da es keine magnetischen Monopole im eigentlichen Sinne gibt. Zu jeder magnetischen Ladung, die durch die Divergenz der Magnetisierung erzeugt wird, gehört auch die Ladung entgegengesetzten Vorzeichens. Die 
von der Magnetisierung erzeugte Ladungsdichteverteilung stellt wiederum die Quelle für ein der Magnetisierung entgegengesetztes magnetisches Feld $\vec{H}_{i}(\vec{r})$ im Innern des Körpers sowie für ein äusseres Feld dar.

Die Lösung der Poissongleichung kann analog zur der des Vektorpotentials unter gleichen Voraussetzungen an die Eindeutigkeit der Lösungen berechnet werden. Das skalare magnetische Potential ergibt sich danach zu

$$
\phi_{M}(\vec{r})=\frac{1}{4 \pi} \int_{-\infty}^{\infty} \frac{\rho_{M}\left(\vec{r}^{\prime}\right)}{\left|\vec{r}-\vec{r}^{\prime}\right|} d^{3} r^{\prime}
$$

und für das magnetische Feld

$$
\vec{H}(\vec{r})=\frac{1}{4 \pi} \int_{-\infty}^{\infty} \vec{\rho}_{M}\left(\vec{r}^{\prime}\right) \cdot \frac{\vec{r}-\vec{r}^{\prime}}{\left|\vec{r}-\vec{r}^{\prime}\right|^{3}} d^{3} r^{\prime}
$$

$\phi_{M}(\vec{r})$ ist also an der Stelle $\vec{r}$ durch die nach ihrem Abstand $\left|\vec{r}-\vec{r}^{\prime}\right|^{-1}$ gewichteten Beiträge der Quellen an den Orten $\vec{r}^{\prime}$ gegeben.

Die beiden dargestellten komplementären Lösungswege über den Wirbel- bzw. den Potentialanteil der Magnetisierung (Gl. 2.1, bzw. 2.2) spiegeln letztendlich den Helmholtzschen Hauptsatz der Vektoranalysis wieder. Ganz allgemein gilt: Ein über einem einfach zusammenhängenden Gebiet mit stückweise glatter Randfläche definiertes Vektorfeld $\vec{F}(\vec{r})$ lässt sich stets additiv zerlegen in einen wirbelfreien $\vec{F}_{\phi}(\vec{r})=-\vec{\nabla} \phi(\vec{r})$ und einen quellenfreien Anteil $\vec{F}_{j}(\vec{r})=-\vec{\nabla} \times \vec{A}(\vec{r})$. Diese Zerlegung ist eindeutig, wenn für die beiden einzelnen Summanden Randwerte vorgegeben werden. Im unendlichen Raum ist dies z.B. durch die oben dargestellte Greensche Funktion gewährleistet [49]. Solch eine Aufspaltung ist somit auch gültig für die Vektorfelder $\vec{B}(\vec{r}), \vec{H}(\vec{r})$ und $\vec{M}(\vec{r})$.

Für die spätere Rekonstruktion der Magnetisierung aus dem Streufeld nach Gl. 2.11 oder aus der Flussdichte mittels Gl. 2.7 sind besonders die Ränder des magnetischen Körpers von entscheidender Bedeutung. Aus den Maxwellgleichungen ergeben sich auf diesen besondere Stetigkeitsbedingungen bzw. Sprungrelationen für die Vektorfelder $\vec{B}(\vec{r}), \vec{H}(\vec{r})$ und $\vec{M}(\vec{r})$.

Für die Normalkomponenten der magnetischen Flussdichte und des magnetischen Streufeldes folgt

$$
\begin{aligned}
& \left(\vec{H}_{a}-\vec{H}_{i}\right) \cdot \vec{n}=\vec{M} \cdot \vec{n}=\sigma_{M} \\
& \left(\vec{B}_{a}-\vec{B}_{i}\right) \cdot \vec{n}=0
\end{aligned}
$$

mit der magnetischen Oberflächenladungsdichte $\sigma_{M}$. Die Indizes $a$ und $i$ stehen dabei jeweils für die Bereiche außerhalb bzw. innerhalb des magnetischen Gebietes. Aus Gleichung 2.12 folgt unter Betrachtung des Potentialanteils der Magnetisierung die Stetigkeit der Normalkomponente der magnetischen Flussdichte, während die des magnetischen Feldes 
einen Sprung um den Betrag von $\sigma_{M}$ macht. Die Stetigkeitsbedingungen der Tangentialkomponenten der Felder ergeben sich zu

$$
\begin{aligned}
\frac{1}{\mu_{0}}\left(\vec{B}_{a}-\vec{B}_{i}\right) \times \vec{n} & =\vec{M} \times \vec{n}=\vec{\jmath}_{M} \\
\left(\vec{H}_{a}-\vec{H}_{i}\right) \times \vec{n} & =0 .
\end{aligned}
$$

Der Ausdruck $\vec{\jmath}_{M}^{s}$ entspricht in diesem Fall einer magnetischen Oberflächenstromdichte. Unter Verwendung des solenoiden Anteils der Magnetisierung ergibt sich nach Gl. 2.14 und 2.15 also für die Tangentialkomponente von $\vec{B}$ ein Sprung um $\left|\vec{\jmath}_{M}^{s}\right|$ in Richtung des Kreuzproduktes, während in diesem Fall die des magnetischen Feldes stetig ist.

Implizit in diesen Bedingungen enthalten ist die Beschreibung der Magnetisierung als eine unstetige Größe. Dies gilt im Gegensatz zu den Feldern $\vec{H}$ und $\vec{B}$ bezüglich seiner Normal- und Tangentialkomponente (2.12 und 2.14). Das Auftreten einer magnetischen Oberflächenladungsdichte bzw. der Oberflächenmagnetisierungsstromdichte ist gerade die Konsequenz aus dieser Unstetigkeit.

Ein Sonderfall der Darstellung tritt ein, wenn in einem endlichen Gebiet die Magnetisierung konstant ist. Die oben genannten Hartmagnetika sind z.B. aufgrund ihrer hohen magnetokristallinen Anisotropie im Bereich kleiner Strukturen und dünner Filme in der Lage genau solche Magnetisierungszustände einzunehmen [50]. In diesem Fall liegt eine Überbestimmtheit des Randwertproblems vor und es reicht die Kenntnis bzw. Vorgabe einer der beiden magnetischen Oberflächendichten aus (äquivalent zur Vorgabe der Tangentialoder Normalkomponente), um die Magnetisierung vollständig zu beschreiben [49]. $\vec{M}$ enthält damit auf dem Rand sowohl einen Rotations- als auch gleichzeitig einen potentiellen Anteil. Die Verwendung von Zusatzgleichungen wie $\vec{\nabla} \times \vec{M}=0$, bzw. $\vec{\nabla} \cdot \vec{M}=0$, zur Eliminierung einer der beiden Anteile führt in diesen Spezialfällen zu falschen Ergebnissen auf dem Rand. In der Literatur kommt es diesbezüglich immer wieder zu unkorrekten Aussagen. Beispielsweise stimmt es nicht, dass der solenoide Anteil prinzipiell kein Streufeld produziert, wie z.B. in $[51,52]$ behauptet. Das Biot-Savart'sche Gesetz zeigt eindeutig, dass senkrecht zur Stromdichte sehr wohl ein Streufeld generiert wird. Desweiteren ist hier eine klare Trennung von Volumen und Rand der Probe vorzunehmen.

Für die Rekonstruktion der Magnetisierung ist diese Randwertproblematik besonders wichtig, da man sich später die Eigenschaft zu Nutze macht, mittels Bestimmung einer der beiden Größen $\sigma_{M}$ bzw. $\vec{\jmath}_{M}^{s}$ auf dem Rand nach Messung des äußeren Streufeldes, die konstante Magnetisierung innerhalb des Gebietes vollständig zu bestimmen.

Abbildung 2.1 verdeutlicht schematisch noch einmal die beiden dargestellten Beschreibungsweisen für den Fall homogen magnetisierter Körper unter Verwendung einer effektiven magnetischen Oberflächenstromdichte $\vec{\jmath}_{M}^{s}(\vec{r})=\vec{M}(\vec{r}) \times \vec{n}$, bzw. magnetischen Oberflächenladungsdichte $\sigma_{M}(\vec{r})=\vec{M}(\vec{r}) \cdot \vec{n}$, die sich aus der Unstetigkeit von $\vec{M}$ auf den Rändern des magnetischen Körpers ergeben. 
(a)
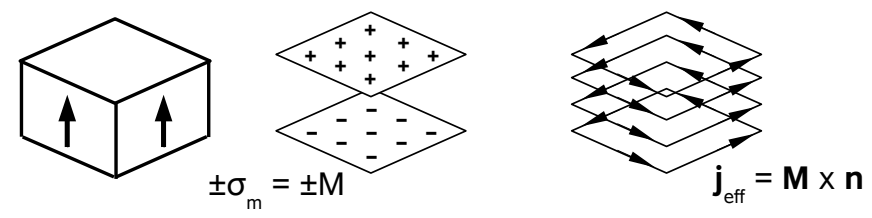

(b)
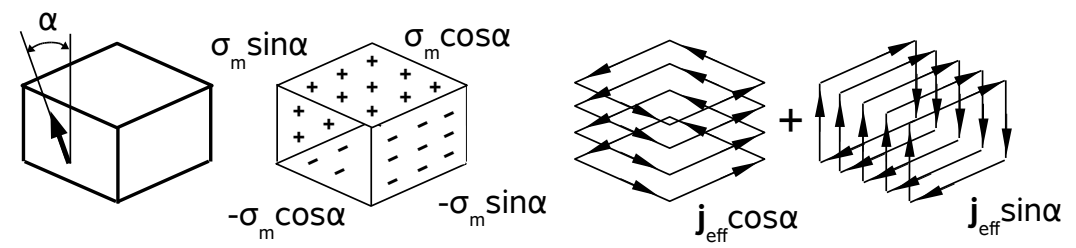

Abbildung 2.1: Darstellung der homogenen Magnetisierung eines prismatischen Körpers mittels magnetischer Oberflächenladungen (Mitte) und magnetischer Oberflächenströme (rechts). (a) Magnetisierung entlang einer Körperachse, (b) beliebig orientierte, schiefwinklige Magnetisierung.

\subsection{Ansatz zur Inversion von Streufeldmessungen 2-dim. Strukturen}

Die Rekonstruktion der Magnetisierung aus Messungen des Streufeldes erfordert die Inversion von Integralgleichungen der Art 2.11 bzw. 2.7. Diese geben die allgemeine Beziehung zwischen der Magnetisierung und dem magnetischen Streufeld bzw. der magnetischen Flussdichte an und stellen eine Klasse von Gleichungen dar, die als Fredholm Integralgleichungen 1. Art bezeichnet werden. Die Inversion solcher Gleichungen ist charakterisiert durch eine intrinsische Instabilität bezüglich nicht-exakter (verrauschter) Daten, da kleine Änderungen im Messsignal verstärkt werden und damit einen großen Einfluß auf die Güte der Lösung haben (siehe Kap. 6.1). In der Literatur wird dies als schlecht-gestelltes Problem bezeichnet, welches in vielen physikalischen Fragestellungen auftaucht $[53,54,55,56]$. Inzwischen existieren eine ganze Reihe von Möglichkeiten der Rauschunterdrückung, um diese Effekte auf ein Minimum zu reduzieren.

Zusätzlich erweisen sich die Integralgleichungen für das volle 3-dim. Problem als uneindeutig. Nach wie vor ungeklärt ist die Frage, wieviel Information über die Streufeldverteilung $\vec{H}(\vec{r})$ tatsächlich bekannt sein muss, um die vollständige Rekonstruktion der Magnetisierungsverteilung $\vec{M}(\vec{r})$ zu gewährleisten.

Alle in der Arbeit verwendeten Abbildungsmethoden sind sensitiv auf die z-Komponente des Streufeldes, so dass die Inversion nur mit Hilfe gewisser Einschränkungen durchzuführen ist. Im Weiteren werden nur dünne magnetische Strukturen betrachtet, die in der sogenannten Dünnschichtnäherung Magnetisierungsverteilungen der Form $\vec{M}(x, y)$ besitzen, also als unabhängig von der z-Koordinate parallel zur Filmnormalen angesehen werden können. Die Messgeometrie, die auch als 2- dim. inverses magnetisches Problem (vgl. Abb. 2.2) bekannt ist [53] und bei der das Messsignal in einer festen Höhe z über der zu untersuchenden Struktur bestimmt wird, reduziert das inverse magnetische Problem auf den Zusammenhang zwischen $H_{z}(x, y, z=$ const $)$ und $\vec{M}(x, y)$ für bekannte Schichtdicken $d$.

Um die Magnetisierung quantitativ bestimmen zu können, muss der Messausschnitt deutlich größer sein als die Strukturgröße des magnetischen Körpers. Damit ist nicht nur 


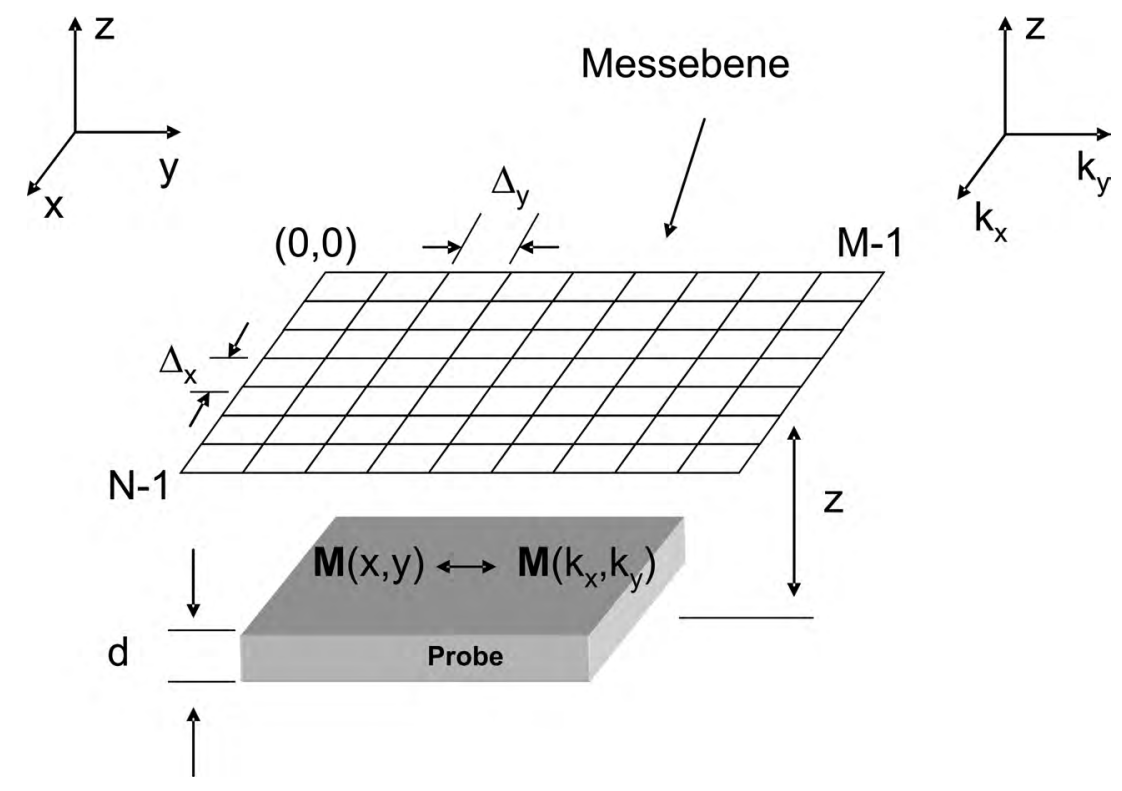

Abbildung 2.2: Schematische Darstellung des inversen magnetischen Problems. Messung von $H_{z}(x, y)$ auf einem rechteckigen Netz mit $N \times M$ Datenpunkten in einer festen Höhe $z$ über der Probenoberfläche. Der Maschenabstand beträgt $\Delta_{x}$, bzw. $\Delta_{y}$ und die Messebene ist parallel zur $(\mathrm{x}, \mathrm{y})$-Ebene. Die Probe ist als dünne Schicht mit der homogenen Dicke $d$ angenähert und besitzt eine von der z-Koordinate unabhängige Magnetisierung $\vec{M}(x, y)$. Die entsprechende Nomenklatur im Fourierraum ist ebenfalls dargestellt.

gewährleistet, dass die Ränder, an denen der Sprung der Magnetisierung die Ladungsdichteverteilung erzeugt, im Bild mit enthalten sind, sondern auch, dass der unmagnetische Außenraum mit $H_{z}=0$ für $\vec{r} \longrightarrow \infty$, der für die Kalibrierung des Messbildes entscheidend ist, mitgemessen wird.

Die Rekonstruktion, so wie sie in dieser Arbeit umgesetzt wird, basiert auf der Verwendung des im vorangegangenen Abschnitts dargestellten Ladungsdichtebildes aus Gründen der besseren Vergleichbarkeit mit einem von Hug et al. [37] erstmals auf magnetische Kraftmikroskopiemessungen angewendeten Inversionsformalismus. Hug verwendet dabei einen Fouriertransformationsansatz, der mittels sogenannter Transferfunktionen die Dekonvolution der senkrechten Komponente der Magnetisierung $M_{z}(x, y)$ und der $H_{z}(x, y)$-Verteilung ausführt. Die Inversion bezüglich der inplane-Komponenten $M_{x}(x, y)$ und $M_{y}(x, y)$ aus $H_{z}(x, y)$ bleiben aber sowohl Hug als auch andere Mitarbeiter der Arbeitsgruppe [38], die diesen Ansatz zur Quantifizierung von MFM-Messungen verwenden, schuldig. Nach meinem besten Wissen gibt es in der Literatur keine experimentell durchgeführte quantitative Berechnung von $M_{x}(x, y)$ - und $M_{y}(x, y)$-Verteilungen aus Messungen der z-Komponente des Streufeldes, was sehr wahrscheinlich auf die Topologie der Messanordnung zurückzuführen ist (Details folgen später). Lediglich die Rekonstruktion der Richtungseigenschaften von $\vec{M}$ wurde z.B. von [57] umgesetzt.

Das Lösen der entsprechenden Integralgleichungen 2.10 bzw. 2.11 erfordert eine Berechnung von Faltungsintegralen im Realraum, was im Allgemeinen einen erheblichen Rechenaufwand zur Folge hat. Aus diesem Grund ist der Weg über den Fourierraum deutlich effektiver (siehe Anhang Gl. A.6). Nach der 2D-Fouriertransformation ergibt sich für das 
skalare magnetische Potential (Nomenklatur analog Abb. 2.2)

$$
\phi_{M}(\vec{k}, z>d / 2)=\int_{-\infty}^{\infty} \rho_{M}\left(\vec{k}, z^{\prime}\right) \frac{e^{-k\left(z-z^{\prime}\right)}}{2 k} d z^{\prime}
$$

mit $k=\sqrt{k_{x}^{2}+k_{y}^{2}}$, der Dicke $d$ der magnetischen Struktur und $z>z^{\prime}$, um der Messanordnung Rechnung zu tragen (Herleitung siehe Anhang). Die Faltung muss nun nur noch in z-Richtung ausgeführt werden.

Aus den Abbildungen 2.1 und 2.2 erkennt man, dass die Messtopologie bei der Streufeldbestimmung spezielle Symmetrieeigenschaften nach sich zieht. Die von der senkrechten Magnetisierungskomponente $M_{z}$ generierten Ladungsdichteverteilungen treten auf Ebenen parallel zur Messebene auf, die von den inplane-Komponenten $M_{x}$ und $M_{y}$ auf Ebenen entsprechend senkrecht dazu. Ausgehend davon, dass $\vec{\nabla} \cdot \vec{M}=\rho_{M}$ grundsätzlich additiv zerlegbar ist, erfordert die unterschiedliche Messtopologie bei der Magnetisierungsrekonstruktion eine Separation der Volumenladungsdichte in einen reinen senkrechten und einen reinen inplane-Anteil. Für $\rho_{M}(\vec{k}, z)$ folgt danach

$$
\begin{aligned}
\rho_{M}(\vec{k}, z) & =\rho_{\perp}(\vec{k}, z)+\rho_{\|}(\vec{k}, z) \\
& =\sigma_{\perp}(\vec{k})\left[\delta\left(z^{\prime}-\frac{d}{2}\right)-\delta\left(z^{\prime}+\frac{d}{2}\right)\right]+\rho_{\|}(\vec{k})
\end{aligned}
$$

mit $\sigma_{\perp}(\vec{k})=\vec{M}(\vec{k}) \cdot \vec{n}$ als der magnetischen Oberflächenladungsdichte, erzeugt von der $M_{z}$-Komponente des Magnetisierungsvektors und $\vec{\nabla} \cdot \vec{M}_{\|}(k)=\rho_{\|}(k)$ von den Komponenten $M_{x}$ und $M_{y}$. Unter der Voraussetzung auftretender Magnetisierungsverteilungen der Art $\vec{M}(x, y, z=$ const $)$ reduziert sich der senkrechte Anteil $\rho_{\perp}$ auf eine Oberflächenladungsdichte, die nur an der Ober- und Unterseite der magnetischen Struktur auftritt und damit durch ein rein im Ortsraum auftretendes Deltafunktional ausgedrückt werden kann. Für die verbleibende parallele Komponente $\rho_{\|}$folgt innerhalb der Schichtdicke $d$ die Unabhängigkeit von $z$. Die Volumenladungsdichte bleibt aber zunächst erhalten.

Setzt man die Ladungsdichteverteilung 2.17 in das Potential 2.16 ein und führt die $z^{\prime}$ Integration aus, so ergibt sich

$$
\phi_{M}(\vec{k}, z)=\alpha(k, d, z)\left[\sigma_{\perp}(\vec{k})+\frac{\rho_{\|}(\vec{k})}{k}\right]
$$

mit

$$
\alpha(k, d, z)=\frac{e^{-k z}}{2 k}\left[e^{\frac{k d}{2}}-e^{-\frac{k d}{2}}\right] .
$$

$\rho_{\|}(k) / k$ stellt nun im eigentlichen Sinne eine inplane-Oberflächenladungsdichte dar, die im Ortsraum auf einem Netz mit diskreten Datenpunkten durch $\sigma_{\|}(x, y)=\rho_{\|}(x, y) \cdot p$ 
gegeben ist, mit $p=\Delta_{x}, \Delta_{y}$ (vgl. Abb. 2.2) als der lateralen Auflösungsgrenze bzw. der Maschenweite der diskreten Datenpunkte.

Das Streufeld über der Probe lässt sich aus dem Gradienten des magnetischen Potentials bestimmen (Gl. 2.8). Für die z-Komponente erhält man nach entsprechender Ableitung von Gleichung 2.18

$$
\begin{aligned}
H_{z}(\vec{k}, z) & =-\frac{\partial}{\partial z} \phi_{M}(\vec{k}, z) \\
& =k \cdot \alpha(k, d, z)\left[\sigma_{\perp}(\vec{k})+\frac{\rho_{\|}(\vec{k})}{k}\right] .
\end{aligned}
$$

Man hat damit im Frequenzraum die Beziehung zwischen den Fourierkoeffizienten des magnetischen Feldes und denen der Ladungsdichten hergeleitet. Dieser mathematische Formalismus über eine Fourierdarstellung von Streufeldern ist in der Literatur durchaus gängig [37, 38, 52, 58, 59, 60, 61, 62], wobei zumeist mit dem senkrechten Fall nur ein Teil des Problems betrachtet wird [37, 52]. Gleichung 2.20 zeigt damit, dass die Messung von $H_{z}$ die Berechnung der Ladungsdichten ermöglicht. Nach Konstruktion der Normalenvektoren kann dann mittels der Sprungrelation $\vec{M} \cdot \vec{n}=\sigma_{M}$ aus den Ladungsdichten eine Rekonstruktion von $\vec{M}(\vec{r})$ erfolgen.

Die Funktion $\alpha$ in Gleichung 2.19 stellt für den dargelegten Formalismus im Frequenzraum eine allgemeine, sogenannte Feldtransferfunktion, dar. In Kapitel 6 soll die explizite Rekonstruktion der Magnetisierung genau auf Grundlage eines solchen, allgemeinen Transferfunktionenformalismus aufgebaut werden. Die Stärke liegt dabei in der Anpassbarkeit an die jeweilige Messmethode. Diese Vorgehensweise erlaubt später eine Kombination der magneto-optischen Messmethode mit der magnetischen Kraftmikroskopie, im Sinne einer möglichen Kalibrierung eines MFM (Umsetzung Kap. 8).

Abschließend sollen kurz die Konsequenzen aus der unterschiedlichen Topologie der Streufeldbestimmung diskutiert werden. Die Separation der Ladungsdichten führt bei der Betrachtung der beiden Grenzfälle rein senkrechter und rein in der Ebene ausgerichteter Magnetisierungsvektoren dazu, dass das Problem aus einer Messgröße zwei unbekannte Anteile bestimmen zu müssen, wegfällt. Daraus ergibt sich auch die Motivation für eine separate Behandlung dieser beiden Spezialfälle in der gesamten Arbeit und bestimmt sogar die Materialauswahl in Kapitel 4 im Hinblick auf die Präparation von Teststrukturen. Für die Rekonstruktion beliebiger Magnetisierungsverteilungen stellt die unterschiedliche Messtopologie jedoch ein Problem dar. Es existiert keine zweite anwendbare Gleichung, um die beiden unbekannten Anteile $\rho_{\perp}$ und $\rho_{\|}$aus der nach Rekonstruktion bekannten Größe $\rho_{M}$ analytisch zu bestimmen. Einzige Möglichkeit erscheint hier die unterschiedlichen Symmetrieeigenschaften der beiden Ladungsdichteanteile auszunutzen. Dies wurde im Verlauf der Arbeit aber nicht mehr umgesetzt. 


\section{Experimentelle Methoden}

Die Rekonstruktion von Magnetisierungsverteilungen aus dem Streufeld der zu untersuchenden Proben erfordert die Verwendung von Strukturen wohldefinierter Form und mit gut bekannten Materialeigenschaften. Diese stellen die grundlegende Basis für die Entwicklung der Inversionsformalismen und damit einer voll quantitativen, lokalen Untersuchung solcher Elemente dar. Als Materialsysteme, auf denen die Umsetzung der Rekonstruktion der Magnetisierung basiert, dienen das FePt-, CoPt- und das PrCo-System. Die präparierten Strukturformen sind im Wesentlichen quaderförmig bzw. rechteckig.

Dieses Kapitel umfasst die kurze Erläuterung aller im Rahmen der Arbeit verwendeten Methoden zur Herstellung und Charakterisierung dieser Teststrukturen. Ausnahme bildet hier nur die magneto-optische Untersuchungsmethode selber, die aufgrund ihrer grundlegenden Bedeutung für diese Arbeit in Kapitel 5 separat behandelt wird.

\subsection{Herstellung hartmagnetischer Filme}

\section{Magnetron-Sputtern von FePt- und CoPt-Filmen}

Die Herstellung der untersuchten $\mathrm{Fe}_{50} \mathrm{Pt}_{50^{-}}$und $\mathrm{Co}_{50} \mathrm{Pt}_{50}$-Filme erfolgte mittels Kodeposition der beiden reinen Elemente in einer Trioden-Magnetron-Sputteranlage im Ultrahochvakuum (UHV) bei einem Restgasdruck von etwa $1 \cdot 10^{-7}$ mbar. Die zur Deposition eingesetzten Trioden-Magnetron-Quellen werden bei einem Argonbasisdruck von $7 \cdot 10^{-3}$ mbar betrieben, so dass die mittlere freie Weglänge nur wenige Zentimeter beträgt. Der Abstand der Quellen gegenüber den Substraten beträgt ca. $23 \mathrm{~cm}$, was eine Thermalisierung der vom Target herausgelösten Atome am Ort des Substrats zur Folge hat. Dadurch besitzen die auftreffenden Atome im Vergleich zu anderen physikalischen Depositionsmethoden (Ionenstrahlsputtern, Laserablation) relativ geringe kinetische Energien (0,5-10 $\mathrm{eV}$ ). Die untersuchten Filme wurden mit einer Rate von $1,6 \AA / \mathrm{s}$ hergestellt, wobei die Schichtdicke typischerweise zwischen $50 \mathrm{~nm}$ und $200 \mathrm{~nm}$ betrug. Details zur Depositionsanlage können [63] entnommen werden.

Die für die magnetischen Untersuchungen gewünschten ausgeprägten Texturen ließen sich durch die Verwendung von epitaxiefähigen, einkristallinen Substraten erreichen. Dazu dienten $10 \mathrm{~mm} \times 10 \mathrm{~mm} \times 0,5 \mathrm{~mm}$ grosse, (001)-orientierte MgO-Substrate. Desweiteren sind hohe Depositionstemperaturen nötig, die zwischen $450{ }^{\circ} \mathrm{C}$ und $730{ }^{\circ} \mathrm{C}$ variierten. Optimale Bedingungen wurden bei Temperaturen zwischen $550{ }^{\circ} \mathrm{C}$ und $650{ }^{\circ} \mathrm{C}$ gefunden. 


\section{Laserablation von $\mathrm{PrCo}_{5}$-Schichten}

Die Herstellung der $\mathrm{PrCo}_{5}$ - Filme mittels gepulster Laserdeposition erfolgte am Institut für Werkstoffforschung (IfW) Dresden in Zusammenarbeit mit der Arbeitsgruppe um Dr. Volker Neu. Die Schichten werden durch alternierenden Abtrag von Elementtargets mit einem KrF-Excimer Laser (Wellenlänge $\lambda=248 \mathrm{~nm}$, Pulsdauer $25 \mathrm{~ns}$, Energiedichte am Target $5 \mathrm{~J} / \mathrm{cm}^{2}$ ) in einer UHV-Kammer hergestellt. Der Basisdruck betrug dabei $10^{-9}$ mbar. Die PrCo5-Schichten wurden bei einer Repetitionsrate von $5 \mathrm{~Hz}$ deponiert, während die zum gewünschten Epitaxieübertrag vom MgO-Substrat auf die dünnen Filme notwendigen Cr-Pufferschichten bei einer Rate von $9 \mathrm{~Hz}$ hergestellt wurden.

Typische verwendete Schichtdicken betragen für die PrCo5-Filme 80 - $100 \mathrm{~nm}$ mit einer zusätzlichen $15 \mathrm{~nm}$ dicken Cr-Puffer- und ebenso dicken Cr-Schutzschicht. Die Depositionstemperatur der Filme liegt während der Herstellung bei $T_{D}^{F i l m}=550{ }^{\circ} \mathrm{C}$ und die der Pufferschichten bei $T_{D}^{\text {Puffer }}=400{ }^{\circ} \mathrm{C}$. Details zur gepulsten Laserdeposition (von $\mathrm{PrCo}_{5}$ - Filmen) finden sich in $[44,64,65,66]$.

\subsection{Probenstrukturierung mittels Elektronenstrahllithographie (EBL)}

Die Strukturierung der hartmagnetischen Filme mittels Elektronenstrahllithographie erfolgte in Zusammenarbeit mit der Physikalisch-Technischen Bundesanstalt in Braun schweig (PTB) und wurde von Herrn Dr. Weimann durchgeführt. Dieser Prozess stellt den entscheidenden Schritt für die Präparation von Strukturen mit wohldefinierter Größe und Form dar.

Die Strukturierung der Filme erfolgte auf zwei unterschiedlichen Wegen. Für die Strukturierung der FePt- und CoPt-Filme wurde ein Positivverfahren (LiftOff-Verfahren) angewendet. Die Verwendung von PolyMethylMethAcrylat (PMMA) als Lithographielack ist dabei Standard (Lösung aus 4\% PMMA (950k) der Markenbezeichnung ARP 671,4 der Firma Allresist). Der Lack wird bei 2000 Umdrehungen/Minute auf die Filme aufgeschleudert, so dass sich eine Schichtdicke von $430 \mathrm{~nm}$ einstellt. Danach wird er für 10 Minuten bei $160{ }^{\circ} \mathrm{C}$ auf einer Heizplatte ausgebacken. Für die Strukturierung werden die Bereiche, die später beim Ionenstrahlätzen geschützt werden sollen, belichtet und mittels einer Lösung aus Isopropylalkohol (IPA): Methylisobutylketone (MIBK) $(3: 1)+1 \%$ Methylethylketone (MEK) herausgelöst. Die Belichtung erfolgte in einem Rasterelektronenmikroskop der Firma Leica EBPG 5000+ bei einer Beschleunigungsspannung von $50 \mathrm{kV}$. In die freigelegten Bereiche wird dann eine $100 \mathrm{~nm}$ dicke Aluminiumätzmaske aufgedampft und der übrige Lack sowie das daraufbefindlichen Alumium, in Aceton anschließend abgelöst. Das Ionenstrahlätzen der Strukturen erfolgte in Göttingen unter $700 \mathrm{eV}$ Ar-Ionen bei einer Rate von 15 nm/Minute. Das restliche Aluminium wurde am Ende durch Ätzen in stark verdünnter Natronlauge entfernt.

Da sich das Pr ebenfalls in Natronlauge löst, muss zur Strukturierung der PrCo5-Filme das PMMA direkt als Ätzmaske dienen. Dieses Negativverfahren erfolgte unter denselben Parametern wie oben geschildert, nur dass die Bereiche, die mit dem Ionenstrahl geätzt werden, diesmal direkt belichtet und entwickelt werden. Details zum Lithographieprozess kann vielen Literaturstellen entnommen werden, beispielhaft sei hier [67] angegeben. 


\subsection{Röntgendiffraktometrie (XRD)}

Die Röntgenbeugung stellt das Standardverfahren zur Analyse des Wachstums und der Texturgüte der hergestellten Filme dar und wurde an zwei verschiedenen Diffraktometern durchgeführt. An einem 4-Kreis-Diffraktometer vom Typ X'Pert der Firma Philips erfolgten die Struktur-, Textur- und Phasenanalysen der FePt-Filme. Dieses Gerät ist mit einer Kobalt-Röntgenröhre $\left(\lambda_{\mathrm{Co}-\mathrm{K}_{\alpha}}=0,17902 \mathrm{~nm}\right)$ ausgestattet und besitzt eine EulerWiege. Die Standardmessungen erfolgten in Bragg-Brentano-Geometrie (sogenannte $\theta$ $2 \theta$ - Geometrie). Im Weiteren ist es möglich, bei fest eingestellter $\theta-2 \theta$ - Reflexbedingung vollständige Polfiguren aufnehmen zu können. In dieser Arbeit wurden lediglich Schnitte durch diese Polfiguren, sogenannte Phi-Messungen, durchgeführt, um eine eventuell in der Filmebene vorliegende Textur zu bestimmen. Dabei wurde bei allen Messungen die $\theta$ $2 \theta$ - Bedingung des (111)-Reflexes eingestellt und die Probe um den $\psi$-Winkel zwischen der (111)- und der (002)-Richtung in einer tetragonalen Einheitszelle verkippt. In dieser Anordnung erwartet man für eine (001)-orientierte Probe vier (111)-Reflexe. Liegen die Reflexe an denselben Winkelpositionen wie die des Substrats bei gleicher Messanordnung, so spricht man bezüglich des epitaktischen Wachstums von einer Würfeltextur (engl.: cube-on-cube).

Desweiteren wurden an den Proben sogenannte Rockingmessungen durchgeführt. Dabei wird die Probe bei festeingestellter $\theta$ - $2 \theta$ - Reflexbedingung um den Winkel $\Omega$ aus seiner idealen Position wenige Grad herausgedreht. Die Halbwertsbreite dieser Rockingkurve ist dann ein Maß für die Verkippung der Körner bezüglich der Substratnormalen und erfasst damit alle (001)-orientierten Körner. Weiterführende Beschreibungen zum verwendeten Texturgoniometer und zu Grundlagen der Texturanalyse kann [68, 69, 70] entnommen werden. Für die Strukturanalyse der CoPt-Filme erwies sich die Kobalt-Röntgenröhre des $\mathrm{X}^{\prime}$ Perts als ungeeignet. Hier war aufgrund von Fluoreszenzanregungen des Kobalts in den Filmen durch die verwendete Co-Strahlung, nahezu kein Streukontrast erkennbar. Deshalb erfolgten alle $\theta-2 \theta$ - Messungen an einem 2-Kreis-Diffraktometer des Typs D500 der Firma Siemens, das mittels einer Kupfer-Röntgenröhre eine Wellenlänge von $\lambda_{\mathrm{Cu}-\mathrm{K}_{\alpha}}=0,15418$ nm erzeugt.

\section{Quantitative Bestimmung des Fernordnungsgrades S}

Die magnetischen Eigenschaften der verwendeten Filme sind maßgeblich von der chemischer Ordnung abhängig, die durch den Fernordnungsgrad S quantifiziert werden kann. Wie in Kapitel 4 dargelegt, besitzen FePt und CoPt im ungeordneten Zustand eine kubisch flächenzentrierte (fcc) Struktur und unterliegen damit den bekannten Auswahlregeln [68]. Liegen die Systeme jedoch in der geordneten $\mathrm{L} 1_{0}$-Struktur vor, dann kommt es für bestimmte verbotene Reflexe nicht zur vollständigen Auslöschung. Abhängig von der Differenz der Strukturamplituden bzw. Strukturfaktoren von Eisen (Kobalt) und Platin bilden sich sogenannte Überstrukturreflexe aus. Da die Ordnungsumwandlung in den beiden Systemen ebenfalls mit einer Gitterverzerrung hin zu einer tetragonal flächenzentrierten (fct) Struktur einhergeht, sind in der fct-Phase sehr viele Reflexe erlaubt. Im Rahmen dieser Arbeit wurden lediglich epitaktisch gewachsene, c-Achsen orientierte Filme hergestellt. Insofern beschränkt sich die weitere Analyse auf die möglichen (001)-Reflexe (mit l=1, 
$2,3)$.

Der Fernordnungsgrad S der geordneten Struktur ist über die Beziehung

$$
S \equiv \frac{\left(r_{F e}-x_{F e}\right)}{y_{P t}}=\frac{\left(r_{P t}-x_{P t}\right)}{y_{F e}}
$$

definiert, wobei $x_{F e(P t)}$ der jeweilige Bruchteil an Eisen(Platin) in der Probe, $y_{F e(P t)}$ der Anteil an Eisen(Platin)-Plätzen und $r_{F e(P t)}$ der Bruchteil an korrekt besetzten Plätzen der beiden Atomsorten ist. Röntgenographisch lässt sich S aus den integrierten Intensitäten der Überstruktur- und der Fundamentalreflexe berechnen. Bei der Bestimmung von relativen Intensitäten müssen aber winkel- und materialabhängige Korrekturterme, wie die Strukturamplitude F, der Lorentz-Polarisationsfaktor LP, der DebyeWaller-Temperaturfaktor D, der Absorptionsfaktor A und das durchstrahlte Volumen $V^{\text {irr }}$, mitberücksichtigt werden. Der Fernordnungsgrad ergibt sich dann gemäß Gleichung 3.2 (Indizes s für Überstruktur- und f für Fundamental-Reflexe)

$$
\begin{aligned}
S^{2} & =\frac{I_{s}}{I_{f}} \cdot \frac{F_{f}\left(\theta_{f}, Z\right) L P_{f}\left(\theta_{f}\right) A_{f}\left(\theta_{f}, \rho\right) D_{f}\left(\theta_{f}, \Theta_{D}, \mathrm{~m}_{\text {atomar }}\right) V_{f}^{\mathrm{irr}}\left(\theta_{f}\right)}{F_{s}\left(\theta_{s}, Z\right) L P_{s}\left(\theta_{s}\right) A_{s}\left(\theta_{s}, \rho\right) D_{s}\left(\theta_{s}, \Theta_{D}, \mathrm{~m}_{\text {atomar }}\right) V_{s}^{\operatorname{irr}}\left(\theta_{s}\right)} \\
& =\frac{I_{s}}{I_{f}} \cdot \Gamma^{F e(C o) P t}\left(\theta_{f}, \theta_{s}\right) .
\end{aligned}
$$

Dabei stellt $\mathrm{Z}$ die chemische Ordnungszahl, $\Theta_{D}$ die Debye-Temperatur, $\mathrm{m}_{\text {atomar }}$ die relative Atommasse und $\rho$ die Dichte der einzelnen Elemente dar, wobei die entsprechenden Größen für die Legierung, also die 50:50 Zusammensetzung, bestimmt werden müssen. Weiterführende Erklärungen zur quantitativen Röntgenbeugung und den einzelnen Korrekturtermen, sowie tabellierte Daten dazu, finden sich in [71, 72]. Bei FePt ergibt sich danach für den (002)-Fundamental- und (001)- bzw. (003)-Überstrukturpeak unter Verwendung von $\mathrm{Co}-\mathrm{K}_{\alpha^{-}}$Strahlung

$$
S=\sqrt{\Gamma_{002 / 001}^{F e P t} \cdot \frac{I_{s}}{I_{f}}}=0,70 \cdot \sqrt{\frac{I_{s}}{I_{f}}} \quad, \text { bzw. } S=\sqrt{\Gamma_{002 / 003}^{F e P t} \cdot \frac{I_{s}}{I_{f}}}=3,18 \cdot \sqrt{\frac{I_{s}}{I_{f}}}
$$

Zur Bestimmung von $\mathrm{S}$ wurden die integrierten Intensitäten aus den $\theta-2 \theta$ - Messungen der zugehörigen Reflexe bestimmt.

\subsection{Rasterelektronenmikroskopie (REM)}

Die Charakterisierung der Oberflächenmorphologie der Filme und daraus präparierten Strukturen mittels Rasterelektronenmikroskopie erfolgten sowohl an einem Stereoscan 360 der Firma Cambridge Instruments als auch an einem LEO Supra 35 Gemini FEG mit Feldemissions-Kathode. Typischer Bereich für die Beschleunigungsspannung an dem 
Sterescan ist 20-40 kV, mit dem man Aufösungen <100 nm erreicht. Sämtliche energiedispersiven Röntgenfluoreszenzuntersuchungen (EDX) bezüglich der chemischen Zusammensetzung der Filme wurden ebenfalls an diesem Gerät durchgeführt. Die Untersuchung der lithographisch präparierten Strukturen erfolgte im zweitgenannten Gerät. Die freistehenden Strukturen sind nach dem Ätzprozess nicht mehr elektrisch leitend miteinander verbunden und befinden sich auf einem isolierenden Substrat $(\mathrm{MgO})$, so dass es bei hohen Beschleunigungsspannungen zu massiven Aufladungserscheinungen kommt. Mit dem Leo Supra ist man in der Lage die Beschleunigungsspannung auf bis zu $1 \mathrm{kV}$ zu reduzieren. Die Auflösung beträgt dann immer noch etwa 2,5 nm (bei $20 \mathrm{kV}$ sind es 1,5 nm). Hiermit konnten bei schräger Betrachtung der Strukturen gute Bildqualitäten ohne gravierende Verzerrungen durch Aufladungserscheinungen erzielt werden.

\subsection{Raster- und magnetische Kraftmikroskopie}

Die Rasterkraft- und die magnetischen Kraftmikroskopieaufnahmen an den FePt- und CoPt-Strukturen wurden in einer Zusammenarbeit mit der Physikalisch-Technischen Bundesanstalt Braunschweig (PTB) von Frau Dr. Sibylle Sievers durchgeführt. Dabei kamen zwei Geräte zur Anwendung. Die Standardanalysen erfolgten an einem Veeco Nanoscope IIIa bei konstantem Abstand. Für die magnetische Abbildung wurden Spitzen der Firma Asylum verwendet, die mit einer $100 \mathrm{~nm}$ dicken CoCr-Schicht versehen sind. Der Krümmungsradius ist nach Spezifikation mit $<10 \mathrm{~nm}$ angegeben und die Koerzivität des magnetischen Materials liegt zwischen 50 - 65 mT. Die Analyse der Oberflächentopographie mit dem Nanoscope erfolgte im kontaktfreien bzw. kontaktarmen Modus, dem sogenannten TappingMode, während für die magnetische Abbildung im kombinierten TappingLiftMode gearbeitet wurde. Die Höheninformation kann dabei verwendet werden, um in einem festem Abstand die Phasenverschiebung der Cantileverschwingung zu messen. Das zusätzlich verwendete Gerät der Firma Surface Imaging Systems (SIS) des Typs Nanostation II erlaubt die Messung der magnetischen Information in einer festen Messhöhe, unabhängig von der Topographie der zu untersuchenden Oberfläche. Dies bietet den Vorteil einer besseren Vergleichsmöglichkeit mit Simulationsrechnungen in einer definierten Höhe über der Probe. Weiterführende Details zur Funktions- und Arbeitsweise sowie dem Aufbau von Kraftmikroskopen kann z.B. einem Übersichtsartikel [73] entnommen werden.

Das Abbildungsprinzip eines magnetischen Kraftmikroskops basiert auf der Wechselwirkung der magnetischen Spitze mit dem Streufeld der Probe, die zu einer zusätzlichen Phasenverschiebung der Cantileverschwingung gegenüber der Anregung führt [74, 75]. Die Kraft F auf die Spitze ist dabei ein Maß für den Gradienten der Wechselwirkungsenergie aus Spitzenmagnetisierung und Probenstreufeld

$$
\vec{F}=-\vec{\nabla} \int_{V_{\text {Spitze }}} \vec{M}_{\text {Spitze }} \cdot \vec{H}_{\text {Probe }} d V
$$

wobei das gesamte magnetische Spitzenvolumen mitberücksichtigt werden muss. Die Phasenverschiebung der in die z-Richtung schwingenden Spitze ist proportional zum Gradienten der Kraft in z-Richtung $\Delta \phi \propto \partial F_{z} / \partial z$. Die Schwierigkeit eine quantitative Information 
gemäß Gleichung 3.3 zu bekommen, liegt daran, dass i. A. die Magnetisierung der Spitze $\left(\vec{M}_{\text {Spitze }}\right)$ nicht bekannt ist und die Wechselwirkung mit dem Probenstreufeld von der zu untersuchenden Strukturgröße abhängt. In der Literatur werden eine Reihe von Methoden und Näherungen diskutiert, um diese Wechselwirkung in einfacher Form zu beschreiben und so die zumeist qualitativen Streufelduntersuchungen quantitativ erfassen zu können. Die sogenannte Monopolnäherung beschreibt die Magnetisierungsverteilung der Spitze durch einen magnetischen Monopol, die Dipolnäherung entsprechend durch einem magnetischen Dipol [76]. Innerhalb dieser stark vereinfachenden Beschreibungen lässt sich die Wechselwirkung mit den magnetischen Spitzen durch definierte Probenstreufelder, die z.B. von stromtragenden Ringen oder Linien [77, 78, 79], sowie durch strukturierte ferromagnetische Filme erzeugt werden [80], quantifizieren. Der Nachteil dieser Art von Kalibrierung liegt darin, dass sie nur für sehr spezielle Feldgeometrien gültig sind und stark von dem Abstand zwischen Probe und Spitze abhängen (siehe auch Kap. 8). Eine fortgeschrittenere Darstellung der Spitzenmagnetisierung verwendet effektive Größen zur Beschreibung [81], also ein effektives Monopol- $q_{\text {Spitze }}^{\text {eff }}$ und Dipolmoment $\vec{m}_{\text {Spitze }}^{\text {eff }}$ gemäß

$$
\vec{F}=\left(q_{\text {Spitze }}^{\text {eff }}-\vec{m}_{\text {Spitze }}^{\text {eff }} \vec{\nabla}\right) \cdot \vec{H}_{\text {Probe }}
$$

Dies führt dazu, dass sowohl die erste Ableitung als auch das Streufeld selbst mitberücksichtigt werden müssen. Problematisch bleibt trotzdem, dass die Größen $q^{\text {eff }}$ und $m^{\text {eff }}$ in effektiven Höhen $z_{q}$ und $z_{m}$ angesetzt werden müssen, wobei die vier Werte stark abhängig von den räumlichen Frequenzen der Probenmagnetisierung, die das externe Streufeld produziert, sind. Es gibt Möglichkeiten die tatsächliche Magnetisierungsverteilung von Spitzen experimentell durch Elektronen-Holographie zu analysieren [82]. Bei beliebigen Probengeometrien bleibt die Problematik der a priori nicht bekannten, komplexen Wechselwirkung (Gl. 3.3) jedoch bestehen.

Eine sehr umfassende Methode zur quantitativen Analyse basiert auf dem Ansatz von Hug et al. über einen allgemeinen Transferfunktionenformalismus, bei der Probleme der oben dargestellten Art auf ein Minimum reduziert werden (vgl. Kapitel 8).

\subsection{SQUID-Magnetometrie}

Im Hinblick auf die magnetische Charakterisierung der FePt-, CoPt- und $\mathrm{PrCo}_{5}$-Filme und deren Verwendung als Modellsysteme für lokale Messmethoden ist eine quantitative Bestimmung der Filmmagnetisierung sehr wichtig. Zu diesem Zweck wurden die präparierten FePt- und CoPt-Filme in einem SQUID- (engl.: Superconducting Quantum Interference Device) Magnetometer analysiert. Diese Methode ermöglicht die sehr präzise, quantitative Messung des magnetischen Moments einer Probe, wobei es sich um eine integrale Messmethode handelt, die gemittelte Werte für die gesamte Probe wiedergibt. Die gewonnenen Daten dienen als Referenzwerte für die lokalen quantitativen Magnetisierungsbestimmungen mittels magneto-optischer als auch magnetischer Kraftmikroskopie.

Die SQUID-Messungen an den FePt- und CoPt-Filmen erfolgten in Zusammenarbeit mit Dr. Sibylle Sievers an der PTB Braunschweig an einem AC-RF-SQUID mit MPMS Controller der Firma Quantum Design durchgeführt. Die Messungen an den $\mathrm{PrCo}_{5}$-Filmen 
wurden am IFW Dresden von Herrn Patra an einem PPMS Vibrationsmagnetometer (VSM) der Firma Quantum Design durchgeführt. Die detaillierten Funktionsweisen eines SQUID- und Vibrationsmagnetometers können z.B. in $[83,84]$ nachgelesen werden. 


\section{Charakterisierung hartmagnetischer Filme und Strukturen}

Dieses Kapitel stellt die Ergebnisse der Untersuchungen der mikrostrukturellen als auch den daraus resultierenden magnetischen Eigenschaften der verwendeten Materialsysteme FePt, CoPt und PrCo dar. Der Fokus liegt dabei auf dem Ziel Strukturen definierter Größe und Form sowie eindeutig ausgezeichneter Magnetisierungsverteilungen zu erhalten, an denen die Rekonstruktion der Magnetisierung aus Streufeldmessungen demonstriert werden soll. Das Kapitel gliedert sich nach den Einsatzmöglichkeiten der verwendeten Materialsysteme die Magnetisierung entlang ausgezeichneter kristallographischer Orientierungen ausrichten zu können. FePt und CoPt dienen als Testsysteme für rein senkrechte Magnetisierungsverteilungen und $\mathrm{PrCo}_{5}$ für rein in der Ebene ausgezeichnete.

\subsection{Eigenschaften und Wachstum von FePt-Filmen}

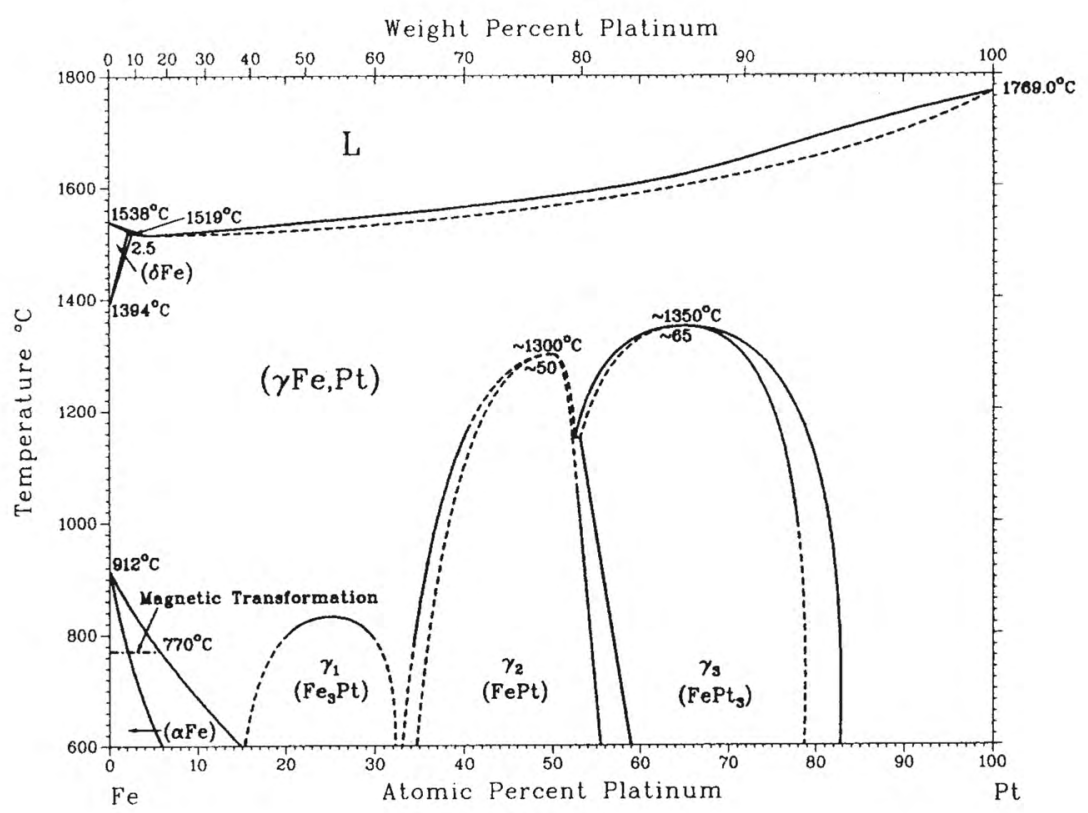

Abbildung 4.1: Phasendiagramm von Fe und Pt [85]

Die Abbildung 4.1 zeigt das Phasendiagramm von $\mathrm{Fe}$ und $\mathrm{Pt}$, das von Co und Pt ist qualitativ sehr ähnlich. Man erkennt über einen großen Bereich das Auftreten von stabilen geordneten Phasen, wobei sich im Folgenden die Analyse auf die $\mathrm{Fe}_{50} \mathrm{Pt}_{50}$-Phase 
beschränkt. Diese besitzt als Gleichgewichtsstruktur bis zu einer Entordnungstemperatur von $1300{ }^{\circ} \mathrm{C}$ die sogenannte $\mathrm{L1}_{0}$-Struktur (Abbildung 4.2). In der c-Richtung ordnen sich dabei reine Fe- und Pt-Atomlagen alternierend an.

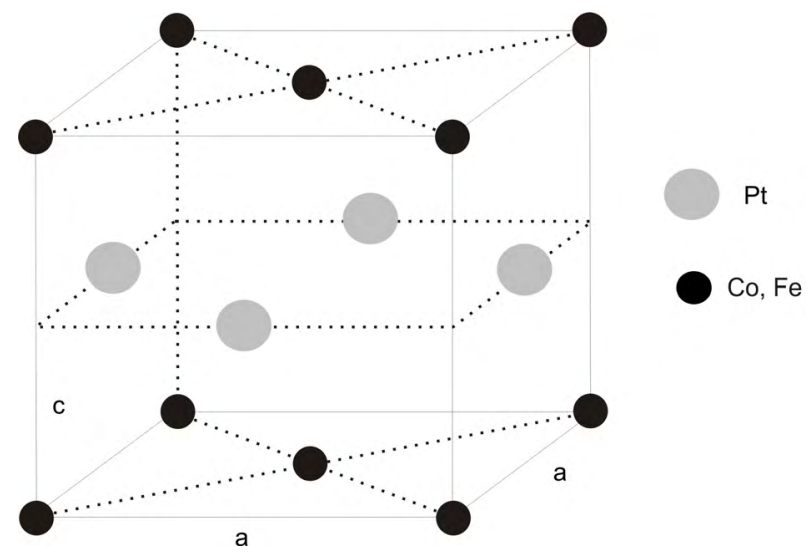

Abbildung 4.2: Geordnete $\mathrm{L}_{1}$-Gitterstruktur für FePt und CoPt

Im ungeordneten Zustand liegt eine kubisch flächenzentrierte (fcc) Einheitszelle vor, die beim Ordnungsübergang in eine tetragonal flächenzentrierte (fct) Zelle mit gestauchter c-Achse und gedehnter a-Achse übergeht. Der Ordnungsübergang ist demnach an eine strukturelle Verzerrung der Einheitszelle gekoppelt(siehe Tabelle 4.1).

\begin{tabular}{|c||c||c|c|}
\hline & $\mathrm{a}_{\mathbf{f c c}}[\mathrm{nm}]$ & $\mathrm{a}_{\mathbf{f c t}}[\mathrm{nm}]$ & $\mathrm{c}_{\text {fct }}[\mathrm{nm}]$ \\
\hline \hline $\mathrm{FePt}$ & 0,380 & 0,385 & 0,371 \\
\hline
\end{tabular}

Tabelle 4.1: Gitterkonstanten von FePt für die fcc- und fct-Phase [86]

Die $\mathrm{L1}_{0}$-Phase besitzt eine sehr hohe uniaxiale magnetische Anisotropie entlang der cAchse. Bei hohem Ordnungsgrad der Filme können dabei Werte für die uniaxiale Anisotropiekonstante $\mathrm{K}_{u}$ von $\approx 7 \cdot 10^{7} \mathrm{erg} / \mathrm{cm}^{3}\left(10^{6} \mathrm{~J} / \mathrm{m}^{3}\right)$ erreicht werden $[42,87,88]$. Das Anisotropiefeld beträgt in hochgeordneten Schichten theoretisch bis zu $\mu_{0} H_{a}=11,6 \mathrm{~T}$.

Diese Eigenschaften können genutzt werden, um Filme und Strukturen zu präparieren, die eine definierte leichte Magnetisierungsachse besitzen indem die tetragonale Achse mittels epitaktischen Wachstums einheitlich ausgerichtet wird [89, 90, 91]. Die Stärke der magnetokristallinen Anisotropieenergie in Filmen zeigt sich im Wesentlichen abhängig von Parametern wie Schichtdicke und Ordnungsgrad der $\mathrm{L} 1_{0}$-Phase und dominiert gegenüber allen anderen magnetischen Energiebeiträgen wie z.B. der magnetischen Formanisotropieenergie oder der Streufeldenergie. Mit sinkendem $\mathrm{S}$ fällt $\mathrm{K}_{u} \mathrm{um}$ mehr als eine Grössenordnung ab [92]. Dies führt trotz c-Achsen orientierten Wachstums solcher Filme zu einem durch die Formanisotropie induzierten Umschlag der Magnetisierung in die Ebene.

Abbildung 4.3 zeigt ein typisches Röntgenbeugungsdiagramm eines bei $650{ }^{\circ} \mathrm{C}$ hergestellten $60 \mathrm{~nm}$ dicken FePt-Films, dessen Zusammensetzung mittels EDX-Analyse im Rahmen der Fehlergrenzen $( \pm 2 \%)$ zu 50 : 50 bestätigt werden konnte. Man erkennt deutlich, dass der Film ein c-Achsen orientiertes Wachstum aufweist. Der bei kleinen Winkeln sichtbare, gut ausgeprägte (001)-Überstrukturpeak lässt auf eine sehr gute Ordnung in dem 


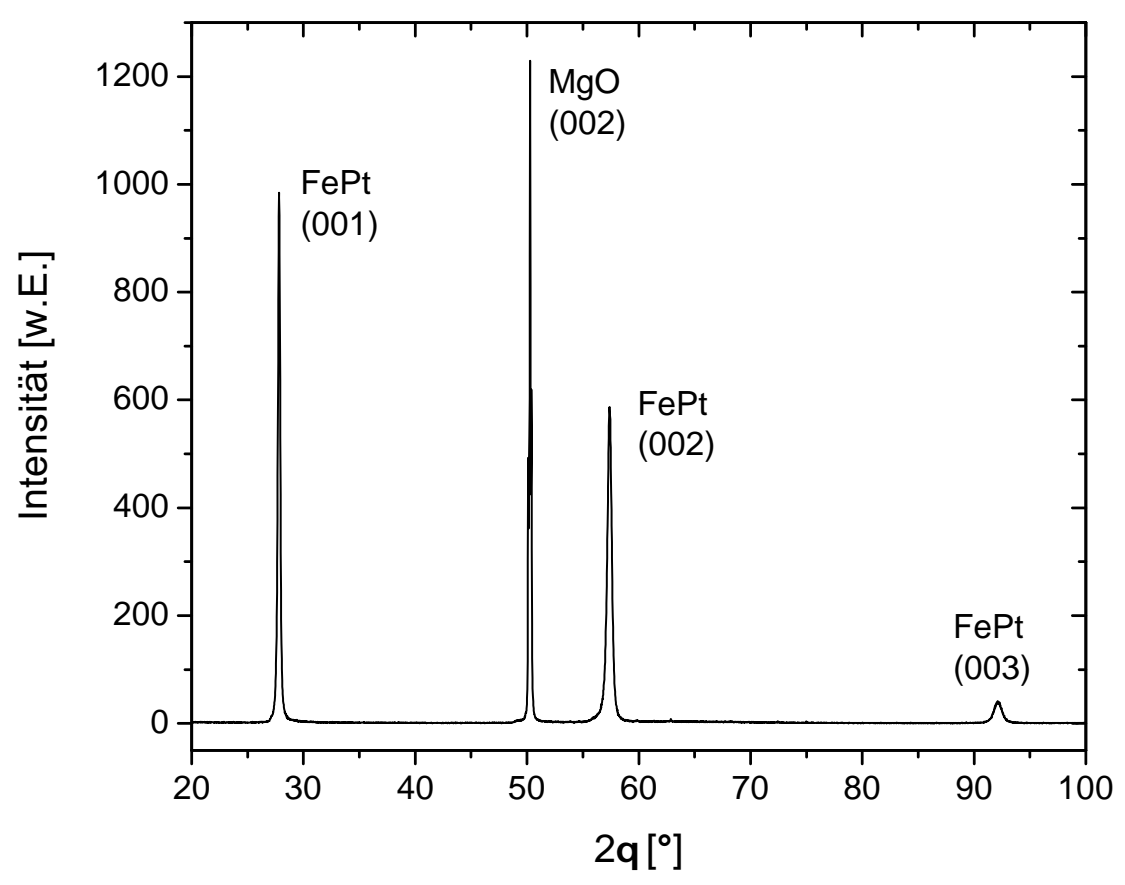

Abbildung 4.3: Röntgendiffraktogramm eines $60 \mathrm{~nm}$ dicken FePt-Films. Die geordnete $\mathrm{L} 1_{0^{-}}$ Struktur mit der senkrecht zur Filmebene liegenden tetragonalen Achse ist an den (001)- und (003)-Überstrukturreflexen eindeutig erkennbar.

Film schließen. Der Fernordnungsgrad S der Schichten kann gemäß Formel 3.2 aus den integralen Intensitäten des (002)-Fundamental- und (001)- bzw. (003)-Überstrukturpeaks berechnet werden. Für den in Abbildung 4.3 dargestellten Film ergibt sich $\mathrm{S}=0,86( \pm 0,2)$. Der recht große Fehler resultiert aus den voneinander abweichenden relativen Intensitäten der beiden Überstrukturreflexe gegenüber dem Fundamentalreflex. Da die (001)- und (003)-Reflexe deutlich unterschiedliche Winkelpositionen haben, können sich hier minimale Fehler beim Probeneinbau in das Röntgendiffraktometer deutlich in den integralen Intensitäten auswirken.

Die Gitterkonstante der tetragonalen Achse ergibt sich nach Auswertung aller (001)Reflexe identisch zu c $=0,3728 \mathrm{~nm}$, woraus beim Vergleich mit den Literaturwerten (siehe Tabelle 4.1) ersichtlich wird, dass hier nur eine sehr geringe Abweichung (0,4\%) vorliegt. Diese kann sowohl auf epitaxievermittelte oder depositionsbedingte Spannungen als auch auf minimale Abweichungen von der äquiatomaren Zusammensetzung zurückgeführt werden. Im ersten Fall würde eine Dehnung des Gitters in Richtung der Schichtnormalen und in der Filmebene eine Querkontraktion vorliegen.

Abbildung 4.4 zeigt eine (111)- $\phi$-Messung des FePt-Films mit vier gut ausgeprägten Reflexen. Eine separate Bestimmung der entsprechenden (111)-Reflexe in $\phi$-Messungen des MgO-Substrates, die bei den gleichen Winkeln auftreten, bestätigen eine Würfeltextur im FePt und die Epitaxierelation ergibt sich zu FePt(001)[100] \| MgO(001)[100]. Die mittlere Halbwertsbreite der vier (111)-Reflexe in Abb. 4.4 ist mit FWHM $_{\text {inplane }}=1^{\circ}$ sehr klein. Ebenfalls durchgeführte Rockingmessungen an den Überstrukturreflexen sowie dem Fundamentalreflex zur Analyse des Verkippungsgrades der tetragonalen Achse 
in dem oben dargestellten FePt-Film ergeben Halbwertsbreiten von $\mathrm{FWHM}_{001}=0,94^{\circ}$, $\mathrm{FWHM}_{002}=0,88^{\circ}$ und $\mathrm{FWHM}_{003}=1,03^{\circ}$ und bestätigen damit die hohe Texturgüte und das sehr gute epitaktische Wachstum der Schichten.

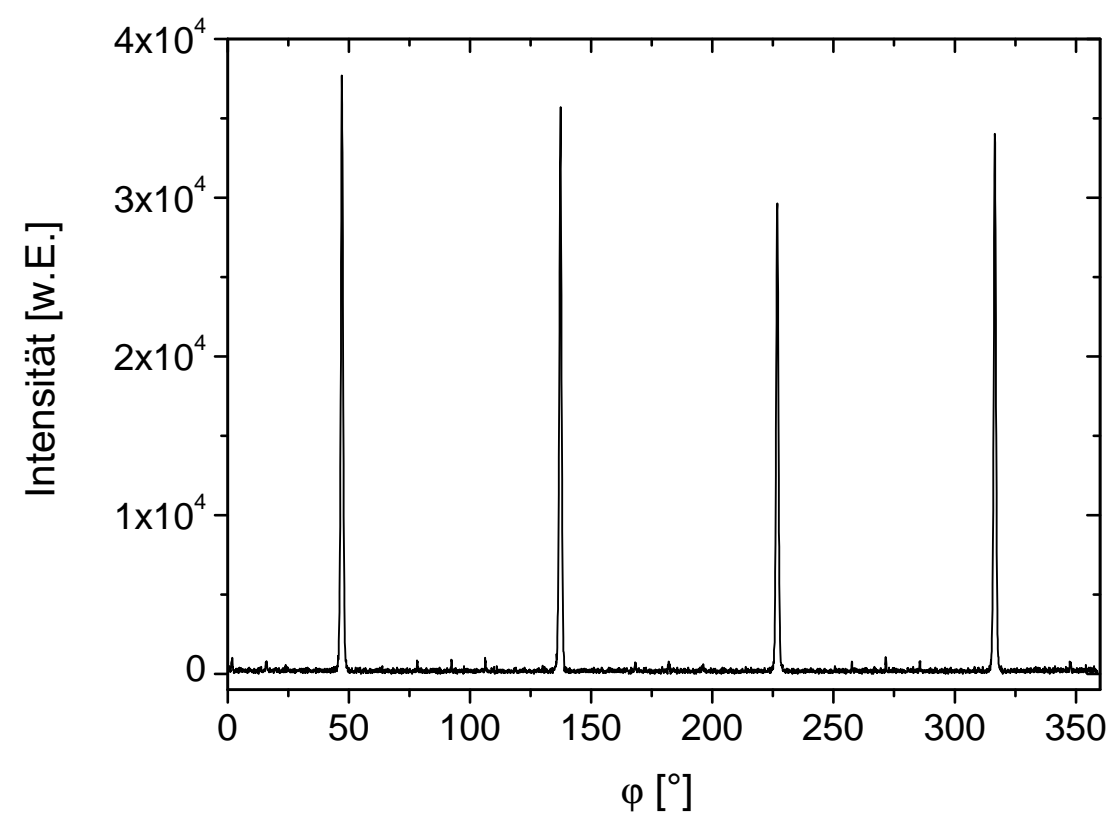

Abbildung 4.4: $\phi$-Messung des (111)-Reflexes eines FePt-Filmes, der bei $650{ }^{\circ} \mathrm{C}$ auf einem (100)-orientierten MgO-Substrat hergestellt wurde.

Ähnliche Ergebnisse an magnetron-gesputterten oder mittels Molekularstrahlepitaxie hergestellten Filmen finden sich in der Literatur [42, 93]. Es existieren allerdings keine Hinweise auf die Ausbildung eines Koinzidenzgitters beim epitaktischen Wachstum der Legierung auf $\mathrm{MgO}$, so dass der Abbau der großen Gitterfehlpassung von $\approx 9 \%$ vermutlich über Versetzungen vermittelt wird. Zhang et al. und Zhao et al. [94, 95] konnten an magnetron-gesputterten FePt-Filmen auf $\mathrm{MgO}(100)$ in transmissionselektronenmikroskopischen Untersuchungen zeigen, dass Versetzungen an der Grenzfläche bzw. in den ersten Lagen der Schicht eingebaut werden. Diese Form der Spannungsrelaxation führt zu einer semikohärenten Grenzfläche, die sich durch einen geringeren Grad an Verspannung auszeichnet [96]. Die Tatsache, dass die a-Achse des FePt knapp vier Prozent länger ist als die c-Achse und damit eine geringere Fehlpassung zum Substrat aufweist, wird als Grund für die Ausrichtung dieser Orientierung in der Ebene angesehen.

\subsection{Eigenschaften und Wachstum von CoPt-Filmen}

Wie bereits erwähnt, besitzt das CoPt-System ein dem FePt sehr ähnliches Phasendiagramm [97]. Allerdings beträgt die Entordnungstemperatur für die $\mathrm{L}_{0}$-Phase lediglich $825^{\circ} \mathrm{C}$. Tabelle 4.2 zeigt die Literaturdaten der Gitterzellen des CoPt.

Die niedrigere Entordnungstemperatur und die leicht abweichenden Gitterparameter zwischen FePt und CoPt erweisen sich im Weiteren als die entscheidenden Faktoren bei der 


\begin{tabular}{|c||c||c|c|}
\hline & $\mathrm{a}_{\text {fcc }}[\mathrm{nm}]$ & $\mathrm{a}_{\text {fct }}[\mathrm{nm}]$ & $\mathrm{c}_{\text {fct }}[\mathrm{nm}]$ \\
\hline \hline $\mathrm{CoPt}$ & 0,375 & 0,381 & 0,370 \\
\hline
\end{tabular}

Tabelle 4.2: Gitterkonstanten von CoPt für die fcc- und fct-Phase [98]

Ausbildung des Gefüges und der Textur in den CoPt-Filmen. Die Tatsache, dass sich die Ordnung beim CoPt bei einer um ca. $475{ }^{\circ} \mathrm{C}$ niedrigeren Temperatur einstellt als beim FePt, zeigt, dass die geordnete $\mathrm{L}_{0}$-Struktur im Fe-Pt-System sehr viel stabiler ist als im Co-Pt [99]. Man kann daher annehmen, dass die mit Defekten assoziierten Energien von Stapelfehlern, Antisite-Defekten, Antiphasen- und Korngrenzen aufgrund des lokalen Bruchs der Ordnung im FePt größer sind als im CoPt. Desweiteren ist das c/aAchsenverhältnis bei FePt mit 0,964 kleiner als im CoPt mit 0,971. Das CoPt muss damit eine geringere Verzerrungsenergie an den Korngrenzen zwischen c- und a-Achsen orientierten Körnern aufbringen. Da die a-Achse im geordneten CoPt gegenüber dem FePt um $0,04 \mathrm{~nm}$ kürzer ist, ist sie auch weniger stark ausgezeichnet und der Misfit zum MgO um $1 \%$ geringer.

All diese Faktoren begünstigen im FePt ein c-orientiertes Wachstum im Vergleich zu CoPt. Daher beobachtet man im CoPt häufiger ein bimodales Wachstum von a- und c-orientierten Anteilen [100] wie exemplarisch an dem Röntgenbeugungdiagramm einer $100 \mathrm{~nm}$ dicken CoPt-Schicht in Abbildung 4.5 an dem aufgespaltenen Fundamentalreflex erkennbar ist. Diese Aufspaltung resultiert aus den unterschiedlichen Gitterkonstanten entlang der tetragonalen Achse und senkrecht dazu (siehe Tabelle 4.1). Das c/a-Verhältnis ergibt sich $\mathrm{zu} \approx 0,98$ und ist im Vergleich mit den Literaturwerten um 0,01 erhöht. Die c- und die a-Orientierung werden gleichberechtigter. Dies bestätigt die Tendenz, dass es für das CoPt leichter erscheint, den Gittermisfit zum Substrat durch eine bimodale Ausrichtung des Kornwachstums mit einer strukturellen Verzerrung hin zum Kubischen abzubauen [101].

Die Volumenanteile der beiden Orientierungen können aus den integralen Intensitäten abgeschätzt werden. Man findet etwa $60 \%$ c-orientierte Körner. Dieses Verhältnis wiesen nahezu alle Filme praktisch unabhängig von der Depositionstemperatur auf. Trotz der Existenz zweier Orientierungen weisen die CoPt-Filme einen hohen Ordnungsgrad auf. Dieser liegt bei typischen Depositionstemperaturen zwischen $600{ }^{\circ} \mathrm{C}$ und $630{ }^{\circ} \mathrm{C}$ in den Schichten bei $S \approx 0,8$.

Eine Möglichkeit, ein epitaktisches Wachstum in [001]-Richtung zu induzieren, liegt in der Verwendung von Pufferschichten. Hierzu wurden zunächst 2-3 nm dicke Platinschichten bei $700{ }^{\circ} \mathrm{C}$ und darauf bei $650{ }^{\circ} \mathrm{C}$ die CoPt-Schichten deponiert. Bei derart dünnen Pufferschichten konnte lediglich bei zwei Filmen ein [001]-orientiertes Wachstum erzwungen werden, mit einem Volumenanteil an a-orientierten Körnern $<5 \%$. Die Verwendung von dickeren Pufferschichten hat zur Folge, dass das Pt in das CoPt hineindiffundiert und somit die äquiatomare Zusammensetzung in den unteren Lagen zerstört, was wiederum zu einer inhomogenen Magnetisierungsverteilung in z-Richtung führt. Da a-orientiertes Wachstum zu einer Magnetisierungskomponente in der Filmebene führt, werden im Folgenden nur solche CoPt-Filme berücksichtigt, die durch die Verwendung der Pt-Pufferschicht eine nahezu reine [001]-Orientierung zeigen. 


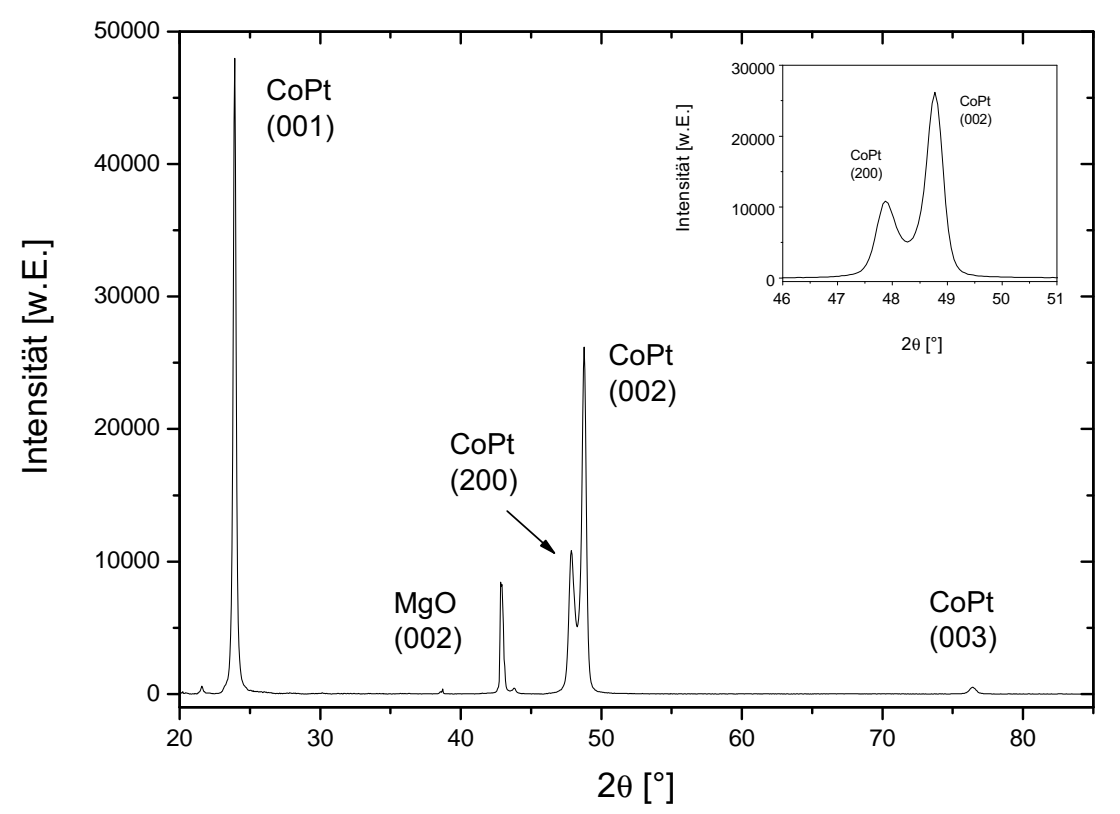

Abbildung 4.5: Röntgendiffraktogramm eines $60 \mathrm{~nm}$ dicken CoPt-Films. Die $\mathrm{L} 1_{0}$-Struktur ist an dem (001)-Überstrukturpeak klar zu identifizieren, allerdings zeigt das CoPt im Vergleich zu FePt ein bimodales c-Achsen und a-Achsen orientiertes Wachstum, erkennbar an dem aufgespaltenen (200)/(002)-Fundamentalreflex, der im Inset noch einmal vergrößerten dargestellt ist.

\subsection{Magnetische Charakterisierung der FePt- und CoPt-Filme}

Zunächst sollen die magnetischen Eigenschaften der FePt-Filme dargestellt werden. Die Tatsache, dass diese Filme ihre leichte Magnetisierungsrichtung durch das epitaktische Wachstum senkrecht zur Filmebene besitzen, wird in Abbildung 4.6 deutlich. Dargestellt ist die SQUID-Messung an einem $60 \mathrm{~nm}$ dicken und bei einer Depositionstemperatur von $\mathrm{T}_{D}=550^{\circ} \mathrm{C}$ hergestellten FePt-Film, der eine zu dem oben beschriebenen Film vergleichbare Texturgüte und einen ähnlichen Ordnungsgrad besitzt. Aufgetragen ist die magnetische Polarisation $P=\mu_{0} M$ gegen das äußere externe Feld. Die Kurven sind um den diamagnetischen Beitrag des MgO-Substrats korrigiert, die unterschiedlichen Entmagnetisierungsfaktoren senkrecht und in der Ebene wurden nicht berücksichtigt.

In der out-of-plane Messung ist der offene, fast rechteckige Verlauf deutlich erkennbar, der typisch für die Messung in Richtung der leichten Magnetisierungsachse bei Hartmagnetika ist. Die Remanenzpolarisation liegt in diesem Film bei $P_{r}=1,31 \mathrm{~T}( \pm 0,04 \mathrm{~T})$ und entspricht bei dem Vergleich mit der Sättigungspolarisation von $P_{s}=1,42 \mathrm{~T}( \pm 0,04 \mathrm{~T})$ einem relativen Wert von $0,93 \mathrm{P}_{r} / \mathrm{P}_{s}$. Dieser im Englischen als squareness einer Hysteresekurve bezeichnete Wert zeigt, dass der aufmagnetisierte Zustand in Remanenz in sehr hohem Maße erhalten werden kann. Der Fehler berechnet sich dabei aus der Annahme einer Schichtdickenungenauigkeit von $2 \mathrm{~nm}$. Der Ummagnetisierungsverlauf ist beispielhaft für einen magnetischen Film mit hoher magnetokristalliner Anisotropie, der durch die Nukleation und das Wachstum von Domänen geprägt ist [69]. Bei kleinen negativen externen 


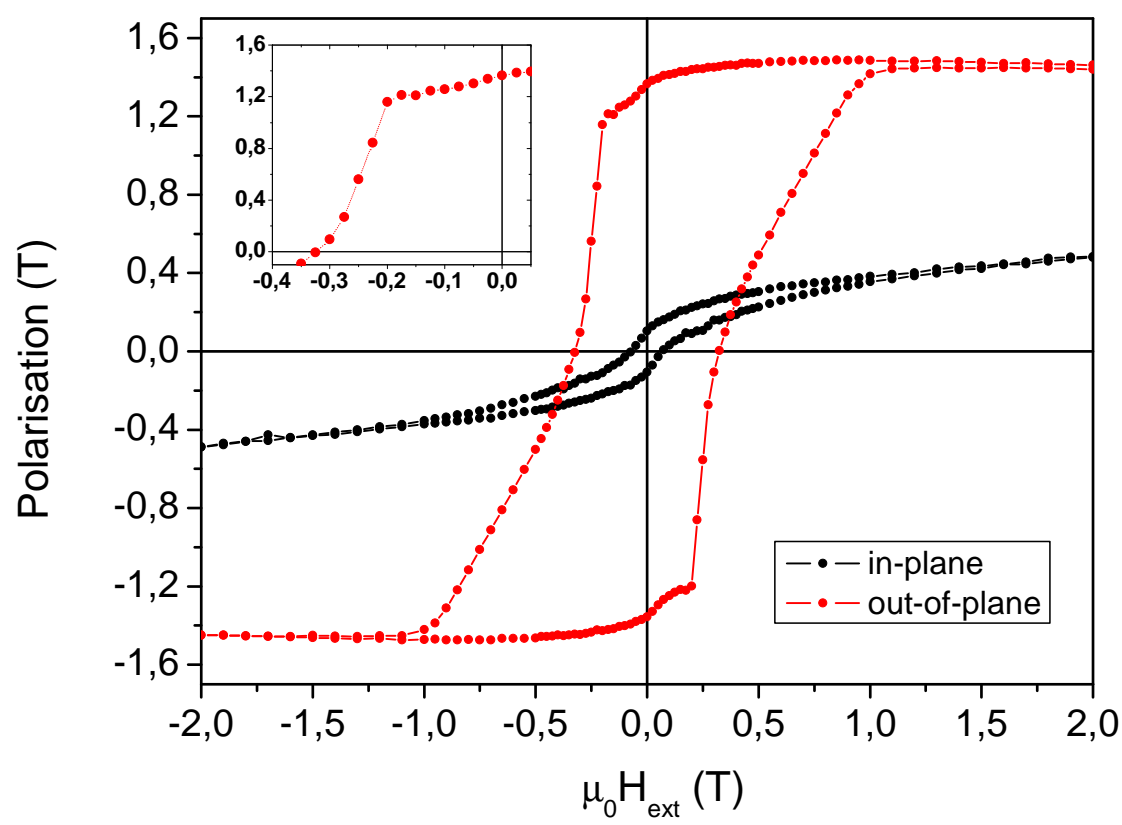

Abbildung 4.6: SQUID-Messung entlang tetragonaler c-Achse (rote Kurve) und a-Richtung (schwarze Kurve) an einem $60 \mathrm{~nm}$ dicken FePt-Film. Der Inset zeigt eine Ausschnittsvergrösserung der out-of-plane Messung im 2. Quadranten.

Feldern erkennt man zunächst einen schwachen Abfall der Polarisation, der auf geringe ungeordnete, weichmagnetische Anteile hindeutet. Diesem folgt ein sprunghafter Abfall, der durch das Nukleieren von Domänen mit entgegengesetzter Magnetisierung ausgezeichnet ist. Dieser tritt bei dem Nukleationsfeld von $\mu_{0} H_{n}=200 \mathrm{mT}$ auf. Nach Überschreiten der Koerzitivfeldstärke, die bei senkrechter Messung $\mu_{0} H_{k}=325 \mathrm{mT}$ beträgt, knickt der Verlauf ab und die Suszeptibilität $\chi=d M / d H$ wird geringer. Dieser Bereich ist besonders durch Domänenwandverschiebung gekennzeichnet und die Änderung der Magnetisierung ist abhängig von der Beweglichkeit dieser Domänenwände, die durch Fehlstellen behindert (engl.: pinning) werden können.

Die Messung in der Filmebene weist eine sehr kleine Hysterese auf, die wieder durch die ungeordneten Anteile erzeugt wird. Generell ist die Zunahme der Polarisation im äußeren Feld, wie für die harte Magnetisierungsrichtung zu erwarten, nur sehr schwach. Ein Maß für die sogenannte magnetische Textur ist das Verhältnis der Remanenzpolarisationen der harten zur leichten Magnetisierungsachse $P_{r}^{\text {hart }} / P_{r}^{\text {leicht }}$. Dieses Verhältnis ergibt sich bei dem FePt-Film zu 0,08. Das Anisotropiefeld kann durch Extrapolation der Messung in der magnetisch harten Richtung ermittelt werden indem der Schnittpunkt mit der Messkurve in die leichte Magnetisierungsrichtung, die bereits für sehr viel kleinere Felder in Sättigung ist, bestimmt wird. Dadurch kann bei dem obigen Film ein Wert von $\mu_{0} H_{a}=9,5 \pm 0,3$ $\mathrm{T}$ abgeschätzt werden. Mittels der Gleichung $K_{u}=0,5 M_{s} \cdot H_{a}$ [102] kann daraus die uniaxiale Anisotropiekonstante berechnet werden, die sich in dem dargestellten Film zu $K_{u}=5,4 \cdot 10^{7} \mathrm{erg} / \mathrm{cm}^{3}$ ergibt.

Die dargelegten Werte sind, analog der strukturellen Messungen im vorherigen Abschnitt, in guter Übereinstimmung mit Literaturdaten [103]. Desweiteren zeigt sich, dass bei gesputterten FePt-Filmen die Remanenz und das Koerzitivfeld nicht nur stark vom Ord- 
4. Charakterisierung hartmagnetischer Filme und Strukturen

nungsgrad und der entsprechend gewählten Depositionstemperatur abhängen [93], sondern bei gleichen Herstellungsbedingungen auch von der Dicke der Filme [104, 105]. Für sehr dünne Filme im Bereich $\leq 40 \mathrm{~nm}$ ergeben sich sehr hohe Energieprodukte. Dies liegt daran, dass die bei geringen Schichtdicken vorliegenden FePt-Inseln noch nicht koalesziert sind und es sich im Wesentlichen um magnetisch entkoppelte, nahezu eindomänige FePtPartikel handelt. Bei Dicken im Bereich $\leq 20-25 \mathrm{~nm}$ vollzieht sich die Ummagnetisierung solcher Teilchen durch kollektive, kohärente Spinrotation während bei Teilchen mit $\mathrm{d} \approx$ 25-40 nm schon inkohärente Rotationsprozesse auftreten. Nach Überschreiten der Perkolationsschwelle $(\approx 45-50 \mathrm{~nm})$ ändert sich der Ummagnetisierungsprozess drastisch und vollzieht sich hauptsächlich durch Nukleation von Domänen und Domänenwandverschiebung.

In dieser Arbeit wurden nur Schichtdicken über der Perkolationsschwelle hergestellt, um bei magneto-optischen Messungen ein möglichst hohes Streufeld zu erzeugen. Damit der Einfluss der Schichtdicke und unterschiedlicher Depositionstemperaturen besser abgeschätzt werden kann, wurden verschiedene Herstellungsserien präpariert. Die Auswertung zu den magnetischen Eigenschaften soll im folgenden dargestellt werden.

Abbildung 4.7 fasst die charakteristischen magnetischen Größen, die sich aus den Hysteresekurven an den FePt-Filmen ergeben zusammen. Abb. 4.7(a) zeigt an der linken Ordinate das Koerzitivfeld und an der rechten das Verhältnis der Remanenz- zur Sättigungspolarisation für die drei verwendeten Depositionstemperaturen bei fester Schichtdicke von $d=60 \mathrm{~nm}$. Man erkennt, dass sowohl $\mu_{0} H_{k}$ als auch die Remanenz bei der niedrigen Depositionstemperatur am geringsten sind. $\mathrm{Ab} T_{D}=550{ }^{\circ} \mathrm{C}$ zeigt sich in den Filmen bereits ein sehr hoher Wert für die Remanenz von $>90 \%$. Die Koerzitivfeldstärke fällt jedoch zu den hohen Temperaturen wieder ab. Depositionstemperaturen zwischen $550{ }^{\circ} \mathrm{C}$ und 650 ${ }^{\circ} \mathrm{C}$ erweisen sich als optimaler Herstellungsbereich. Abb. 4.7(b) gibt die beiden gezeigten Größen bei fester $T_{D}$ als Funktion der Schichtdicke wieder. Deutlich erkennbar ist, dass $\mu_{0} H_{k}$ mit zunehmender Schichtdicke fällt. Für die Remanenz zeigt sich der rechteckige Verlauf der Hysterese nur bei geringen Schichtdicken. Dies erklärt sich aus den bereits oben erwähnten Gründen.

a.)

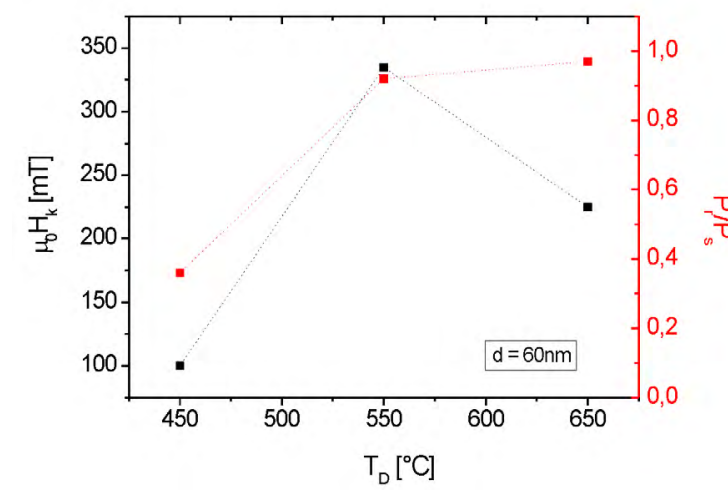

b.)

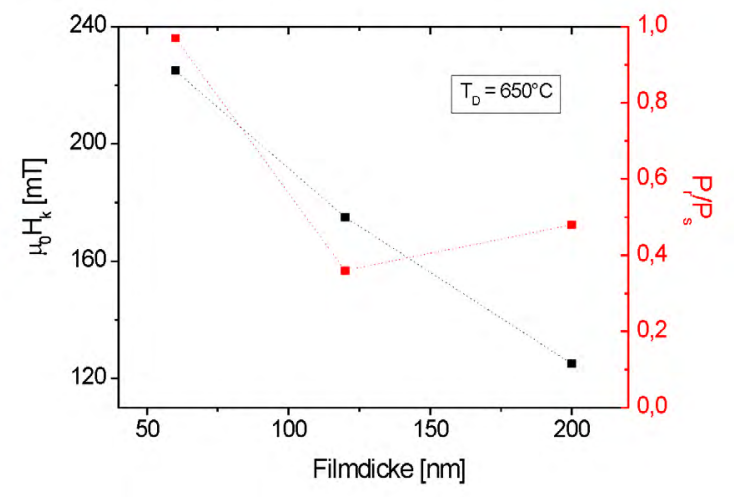

Abbildung 4.7: (a) Linke Ordinate: Koerzitivfeld bei fester Schichtdicke von $60 \mathrm{~nm}$ als Funktion der Depositionstemperatur. Rechte Ordinate: Squareness gegen Depositionstemperatur. (b) Linke Ordinate: Koerzitivfeld gegen Schichtdicke bei fester Depositionstemperatur. Rechte Ordinate: Squareness als Funktion der Schichtdicke. 
Die Anisotropiefelder der Filme für $T_{D}>550^{\circ} \mathrm{C}$ liegen bei sämtlichen Schichtdicken zwischen $9 \mathrm{~T}<\mu_{0} H_{a}<11 \mathrm{~T}$, woraus sich für die uniaxialen Anisotropiekonstanten ein Bereich von $5-6,5 \cdot 10^{7} \mathrm{erg} / \mathrm{cm}^{3}$ ergibt. Dies bestätigt die sehr guten magnetischen Charakteristika der hergestellten FePt-Filme.

Demgegenüber zeigen die SQUID-Messungen an den CoPt-Filmen mit a-orientierten Anteilen rechteckige Hysteresekurven für beide Feldorientierungen. Die magnetisch leichte Richtung kann entsprechend $[001]_{\mathrm{CoPt}} \|[100]_{\mathrm{MgO}},[010]_{\mathrm{MgO}},[001]_{\mathrm{MgO}}$ in allen drei Raumrichtungen liegen. Eine über große Volumina mittelnde Messmethode wie das SQUID führt in solchen Fällen zu einer magnetischen Textur nahe 1. Weitere Details zu den Hysteresekurven und dem Ummagnetisierungsverhalten der CoPt-Filme mit Pt-Pufferschicht sind in Kapitel 7.3 im Vergleich zu strukturierten Filmen dargestellt.

\subsection{Wachstum und Eigenschaften von $\mathrm{PrCo}_{5}$-Filmen}

Dieses Kapitel stellt die wichtigsten Grundlagen zu dem Pr-Co-System dar und gibt einen zusammenfassenden Überblick über die durchgeführten strukturellen und magnetischen Untersuchungen zu den wie hergestellten Filmen (siehe Kap. 3.1).

In der Zusammensetzung 1:5 bildet das Pr-Co-System eine geordnete Kristallstruktur aus, dessen Prototyp die hexagonale $\mathrm{CaCu}_{5}$-Struktur ist. Die Einheitszelle für $\mathrm{PrCo}_{5}$ weist nach [106] Gitterkonstanten von $\mathrm{a}=0,5012 \mathrm{~nm}$ und $\mathrm{c}=0,3984 \mathrm{~nm}$ auf. Dieser große Unterschied in den Längen der hexagonalen c-und der a-Achse ist verbunden mit einer starken uniaxialen magnetokristallinen Anisotropie in Richtung der kristallographischen c-Orientierung mit einer Anisotropiekonstante im Bereich von $K_{u} \approx 8 \cdot 10^{7} \mathrm{erg} / \mathrm{cm}^{3}$ und einer Sättigungsmagnetisierung von $\mu_{0} M_{s}=1,24 \mathrm{~T}$ [44]. Zwingt man nun das System mit seiner leichten Magnetisierungsachse durch ein geeignetes epitaktisches Wachstum in die Filmebene, so erhält man magnetisch hochanisotrope Filme [107], deren Koerzitivfeldstärken im Bereich von bis zu $\mu_{0} H_{k} \approx 2,5 \mathrm{~T}$ liegen und theoretische Anisotropiefelder von $\mu_{0} H_{a}=14,5 \mathrm{~T}$ haben.

Das epitaktische Wachstum kann mittels einer Cr-Pufferschicht auf $\mathrm{MgO}(100)$ induziert werden, wobei das Cr gleichzeitig als Diffusionsbarriere für das Praseodym dient, das zur Oxidation mit dem Substratsauerstoff neigt [108]. Die optimalen Depositionstemperaturen für die Cr-Pufferschicht liegen bei $400{ }^{\circ} \mathrm{C}$ und für den $\mathrm{PrCo}_{5}$-Film bei $500-550{ }^{\circ} \mathrm{C}$ [44]. Das Röntgendiffraktogramm eines $100 \mathrm{~nm}$ dicken unter diesen Bedingungen hergestellten Films ist in Abbildung 4.8 gezeigt.

Man erkennt ein (110)-orientiertes Wachstum des Pr-Co auf der 15 nm dicken (200)orientierten Cr-Pufferschicht, die wiederum epitaktisch auf dem $\mathrm{MgO}(100)$ aufgewachsen ist. Texturmessungen bestätigen sowohl im $\mathrm{Cr}$ als auch im $\mathrm{PrCo}_{5}$-Film eine Ausrichtung der Körner in der Ebene. Die Pufferschicht wächst in einer $45^{\circ}$-Rotationsepitaxie auf dem $\mathrm{MgO}$ auf. Das Pr-Co wächst mit der hexagonalen c-Achse in der Ebene gegenüber der Cr-Unterlage erneut um $45^{\circ}$ gedreht auf. Aufgrund der vierzähligen Symmetrie der [100]-Orientierung des MgO-Substrats und auch der Cr-Pufferschicht, liegt die c-Achse des $\mathrm{PrCo}_{5}$ damit in den zwei zueinander orthogonalen aber gleichberechtigten $\mathrm{MgO}[100]-$ und 


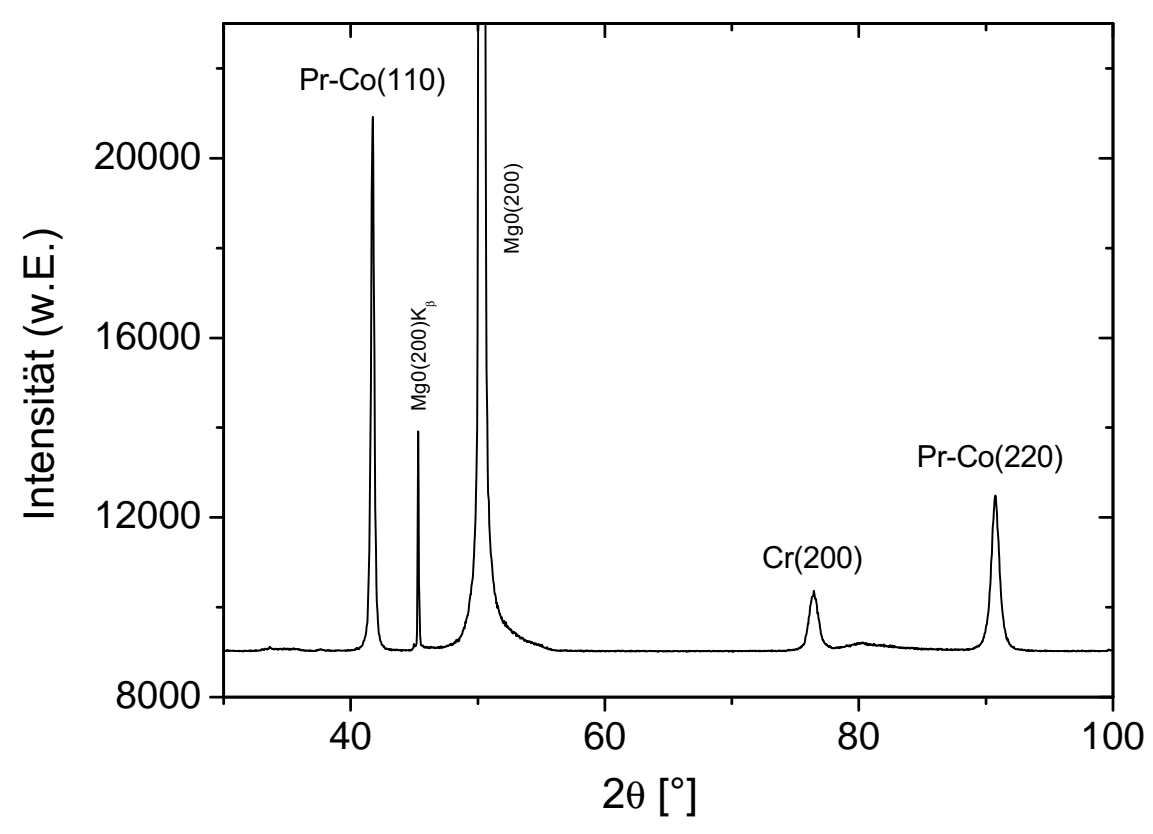

Abbildung 4.8: Röntgendiffraktogramm eines 100 nm dicken $\mathrm{PrCo}_{5}$-Filmes

[010]-Richtungen, also parallel zu den Kanten des Substrats. Die komplette Epitaxierelation beträgt für diesen Schichtaufbau Pr-Co(110)[001]\| $\mathrm{Cr}(100)[011] \| \mathrm{MgO}(100)[100]$ und $\operatorname{Pr}-\mathrm{Co}(110)[001]\|\mathrm{Cr}(100)[01 \overline{1}]\| \mathrm{MgO}(100)[010]$.

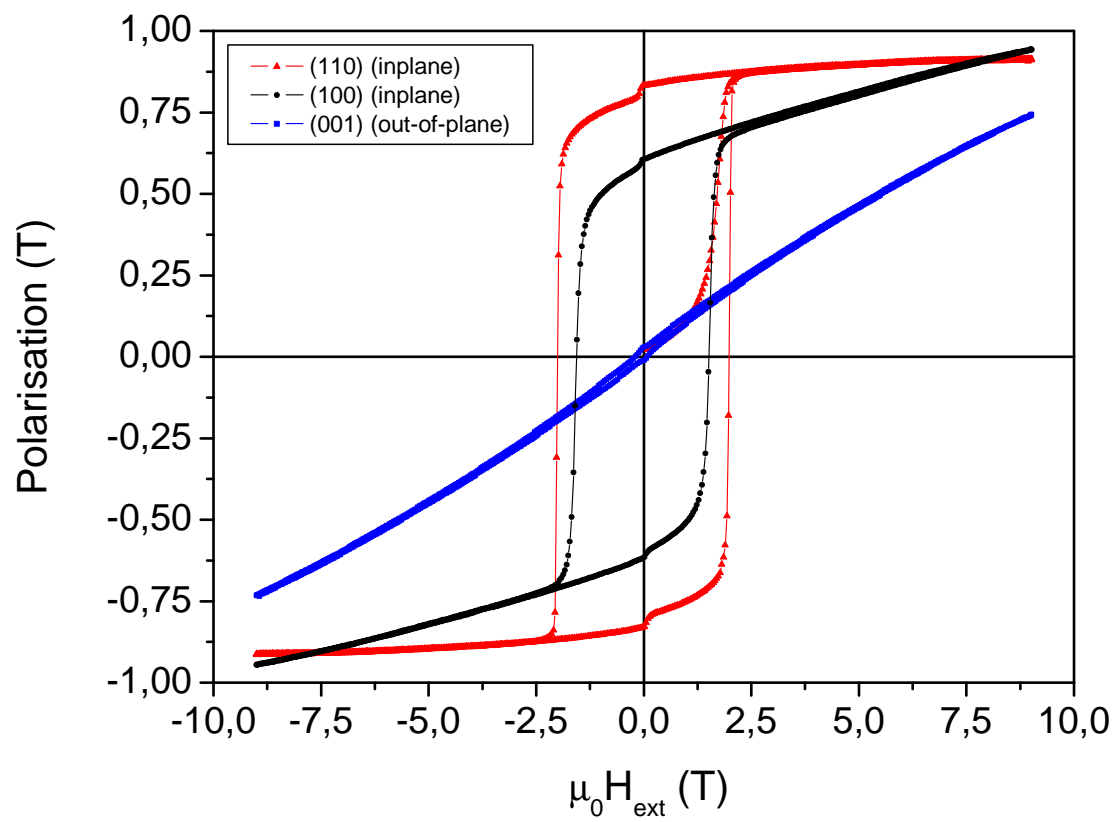

Abbildung 4.9: Hysteresekurven des $\mathrm{PrCo}_{5}$-Filmes entlang den drei angegebenen kristallographischen Richtungen.

Für die magnetischen Eigenschaften hat die Ausrichtung der leichten Magnetisierungs- 
achse in der Ebene charakteristische Auswirkungen. Durch das Ausrichten der c-Achse entlang beider Kanten des MgO-Substrats erhält man eine biaxiale Verteilung der leichten Magnetisierungsrichtung innerhalb der Filmebene. Abbildung 4.9 zeigt die Polarisationsmessung des oben dargestellten $\mathrm{PrCO}_{5}$-Filmes. Bei senkrechter Orientierung des äußeren Feldes gegenüber den leichten Magnetisierungsachsen bildet sich ähnlich wie beim FePt der hartmagnetische Verlauf mit einer sehr schmalen und flachen Hysterese aus. Aufgrund der beiden senkrecht zueinander stehenden, leichten Magnetisierungsachsen in der Filmebene, erhält man einen gemischten Magnetisierungsverlauf bei Messung der Polarisation entlang einer dieser Kanten. Die Kurve in [100]-Richtung weist sowohl eine rechteckige Hysterese mit einer Remanenzpolarisation von $P_{r}=0,61 \mathrm{~T}$ und einer Koerzitivfeldstärke $\mu_{0} H_{k}=1,3 \mathrm{~T}$ auf. Zu höheren externen Feldern überlagert sich der hartmagnetische Beitrag der dazu senkrechten leichten Richtung und führt zu dem weiteren nahezu linearen Anstieg der Polarisation. Bei Messung in [110]-Richtung tritt ein Winkel von $45^{\circ} \mathrm{zu}$ beiden leichten Achsen auf. Bei Ausrichtung der Spins entlang der Kanten des Substrats folgt von beiden Richtungen als Messbeitrag die entsprechende Projektion auf die Diagonale. Die Remanenzpolarisation $P_{r}=0,83 \mathrm{~T}$ und die Koerzitivfeldstärke von $\mu_{0} H_{k}=2 \mathrm{~T}$ erweisen sich auch deutlich höher als bei der Messung entlang der [100]-Richtung.

Die Streufeldverteilung von $\mathrm{PrCO}_{5}$-Quadraten ist je nach Orientierung auf dem Substrat und relativ zum externem Feld durch die ausgezeichneten leichten Magnetisierungsachsen verschieden. Für das Verständnis der in den folgenden Kapiteln gezeigten experimentellen Streufeldverteilungen sind die unterschiedlichen Möglichkeiten in einer schematischen Darstellung in Abbildung 4.10 gezeigt. Abb. 4.10(a) und (b) stellen die einfachen Fälle reiner $M_{x^{-}}$und reiner $M_{y}$-Komponenten dar. Das Streufeld zeigt dabei direkt in x- und y-Richtung und die Kanten des Quadrates sind parallel zu den Substraträndern. Abb. 4.10(c) zeigt die Ladungsverteilung an den Rändern des Quadrates bei einem externen Feld, das in die Strukturdiagonale weist. Hierbei treten beide Magnetisierungskomponenten und damit an allen Rändern Ladungen auf. Die SQUID-Messung in [110]-Richtung liefert eine Remanenz von $P_{r}=0,83 \mathrm{~T}$. Dies ergibt sich aus der Summe der Projektionen von $M_{x}$ und $M_{y}$ auf die Diagonale. Abbildung 4.10(d) zeigt den analogen Fall zu (c) mit dem Unterschied, dass die Strukturränder gegenüber den Substratkanten um $45^{\circ}$ gedreht präpariert wurden. Bei diagonal angelegtem Feld bekommt man nun ein überlagertes Streufeld. An den beiden senkrecht zum Feld zeigenden Kanten erhält man eine destruktive Überlagerung der Ladungen und kein Nettostreufeld.

\subsection{Strukturierung hartmagnetischer Schichten}

Für die Ziele dieser Arbeit ist die Strukturierung der Filme zu kleinen Einheiten mit wohl definierten Dimensionen und Abständen von besonderer Bedeutung. Das Augenmerk liegt dabei auf der Kantenqualität, da diese auch einen merklichen Einfluss auf die Streufelder haben.

Abbildung 4.11 zeigt rasterelektronenmikroskopische Aufnahmen von CoPt-Strukturen, wie sie nach der Elektronenstrahllithographie und dem Ionenstrahlätzprozess vorliegen. Die Aufnahmen (a) und (b) zeigen jeweils eine Struktur mit den Kantenlängen $5 \mu \mathrm{m}$ und $1 \mu \mathrm{m}$. Die beiden Quadrate weisen eine gute Qualität der Kanten auf. Die leicht abgerundeten Ecken haben einen Krümmungsradius im Bereich von etwa $30 \mathrm{~nm}$ und die Seiten 
a.)

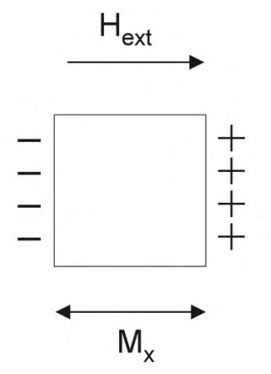

c.)

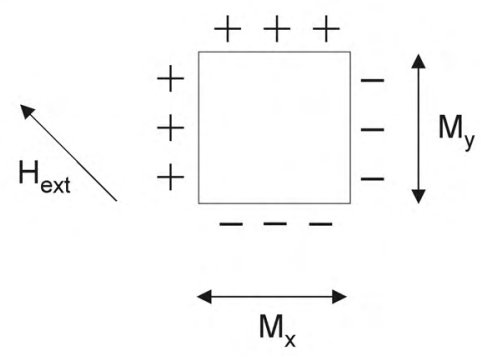

b.)

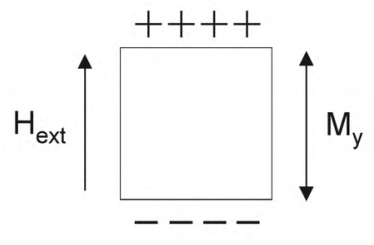

d.)

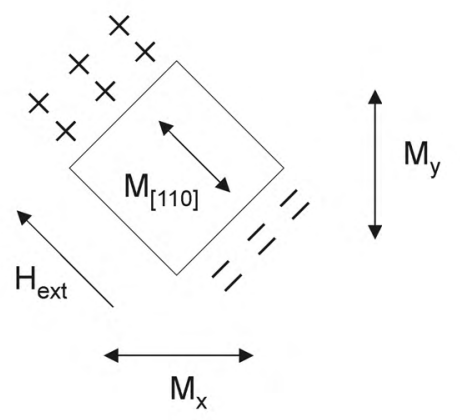

Abbildung 4.10: Resultierende Ladungsverteilungen für $\mathrm{PrC}_{5}$-Strukturen je nach Orientierung ihrer Kanten zum Substrat bei unterschiedlichen externen Feldrichtungen.

besitzen eine minimale Wölbung. Auch erkennbar ist eine schwache Kantenrauigkeit. Diese Effekte können im Aspektverhältnis zu den Kantenlängen aber vernachlässigt werden, da sie deutlich kleiner als das Aufösungsvermögen der magneto-optischen Abbildungsmethode sind. Zudem zeigen die in Kap. 5.5 vorgestellten Streufeldsimulationen keinen signifikanten Einfluss. Abb. 4.11(c) zeigt ein Übersichtsbild einer strukturierten Anordnung von Quadraten mit $1 \mu m$ Kantenlänge und Abstand. Das Feld besteht aus wohlseparierten Einheiten ohne Lackreste oder nur teilweise abgetragenen CoPt-Bereichen.

\subsection{Hartmagnetische Strukturen als Modellsysteme}

Die Auswahl der zur weiteren magnetischen Untersuchung mittels der MOIF-Methode verwendeten Filme ordnet sich bestimmten Voraussetzungen unter. Es geht dabei zum einen um die Herstellung von Filmen mit sehr hohem Energieprodukt, also einer möglichst hohen Remanenzmagnetisierung bei gleichzeitig sehr hohem Koerzitivfeld. Diese Filme sind wichtig, da sie sich aufgrund der definierten Magnetisierungskonfiguration zur Verwendung als Modellsysteme eignen. Zum anderen muss gewährleistet sein, dass die Konfiguration von den verwendeten Abbildungsmethoden nur minimal beeinflusst werden und nicht zu große bzw. zu kleine wohldefinierte Streufelder erzeugen. Für diesen Zweck stellen die FePt-Filme als Repräsentant für eine rein senkrechte Magnetisierungkonfiguration bis zu einer Schichtdicke von $60 \mathrm{~nm}$ und Depositionstemperaturen von $550^{\circ} \mathrm{C}<T_{D}<650^{\circ} \mathrm{C}$ die optimalen Teststrukturen dar. Ihre sehr hohe Texturgüte, gekoppelt an einen hohen Ordnungsgrad resultieren in geeigneten magnetischen Eigenschaften mit einer ausgeprägten senkrechten Vorzugsachse der Magnetisierung und sehr hohen Remanenzen bei nicht zu 
a.)

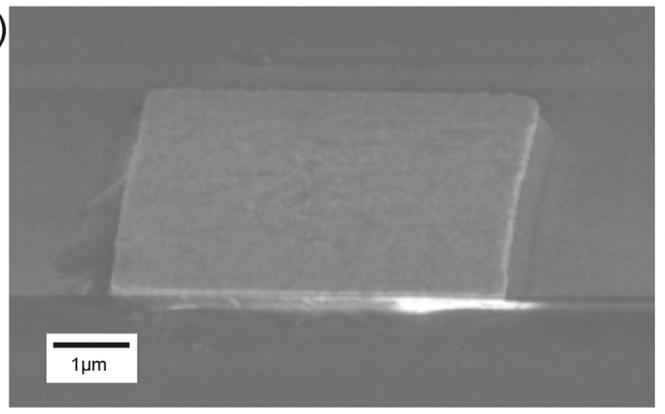

c.)

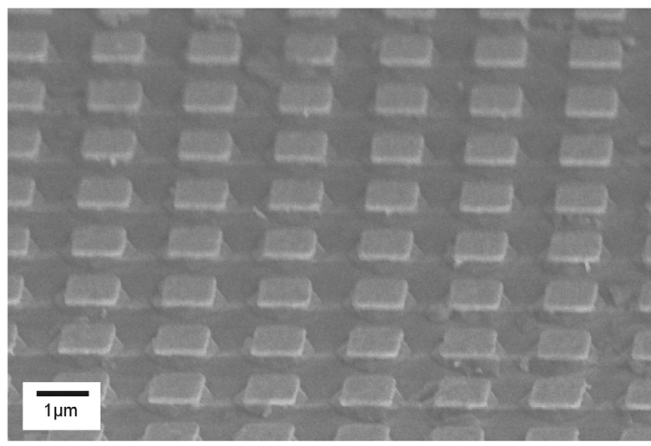

b.)

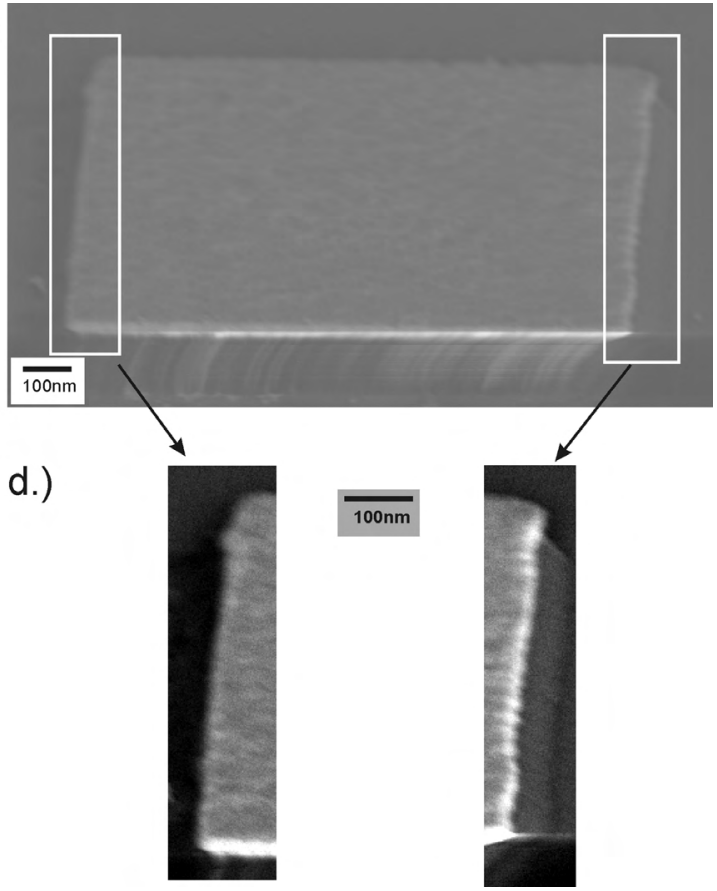

Abbildung 4.11: Rasterelektronenmikroskopische-Aufnahmen von einzelnen CoPt-Quadraten mit Kantenlänge (a) $5 \mu \mathrm{m}$ und (b) $1 \mu \mathrm{m}$ und (c) eines Strukturfeldes mit $1 \times 1 \mu \mathrm{m}^{2}$-Quadraten mit $1 \mu \mathrm{m}$ Abstand. Die Bilder sind unter einem Winkel von etwa $50^{\circ}$ aufgenommen worden, um Aufladungserscheinungen und Überstrahleffekte (wie in (a) und (b) schwach erkennbar) an dem isolierenden MgO-Substrat zu minimieren. (d) Vergrößerung der Kanten des $1 \mu \mathrm{m}$ Quadrates aus (b).

geringen Schichtdicken. CoPt erweist sich als wenig geeignet, da das bimodale Wachstum zu einer wenig definierten Magnetisierungskonfiguration führt. Auch die Remanenz der Filme mit Pufferschicht ist nicht so hoch wie beim FePt, es treten damit mehr Gegendomänen auf. Die Grundlage der Kalibrierung für den senkrechten Fall stellen somit die FePt-Strukturen dar.

Das $\mathrm{PrCo}_{5}$-System erweist sich aufgrund der hartmagnetischen Eigenschaften ebenfalls als geeignet für eine Untersuchung der Beziehung des Streufeldes und der zugrundeliegenden Magnetisierung und dessen Rekonstruktion bei vollständiger Ausrichtung in der Ebene. Die geschilderte Flexibilität bei der Ausrichtung der leichten Achse der Magnetisierung in unterschiedlichen kristallographischen Orientierungen in diesem Materialsystem kann zusätzlich zur Realisation komplexerer Magnetisierungsverteilungen genutzt werden.

Die Strukturierung der Proben in definierte Einheiten mit guter Kantenqualität, sowohl in deutlich voneinander separierte Einzelstrukturen als auch in Strukturfelder mit kleinem Abstand, stellt die Grundvoraussetzung für die Eichung auf verschiedenen Längenskalen dar. Hier zeigt sich eine, bezüglich der experimentellen Auflösung der Abbildungsmethode, absolut ausreichende Qualität.

Die Charakterisierung der hartmagnetschen Filme, an denen Ummagnetisierungsmechanismen studiert werden sollen, ordnet sich einer zusätzlichen Limitierung durch die Messapparatur unter. Die externen magnetischen Felder, die in dem Messaufbau realisiert werden können, sind auf $300 \mathrm{mT}$ begrenzt (siehe Kap. 5.2). Um die Proben in-situ ummagnetisieren zu können, sollten die Koerzitivfelder der zu untersuchenden Strukturen 
möglichst kleiner sein als dieser Wert. Die verwendeten $\mathrm{PrCo}_{5}$-Strukturen erweisen sich aufgrund der sehr hohen Koerzitivfelder aber dafür nicht mehr geeignet. Der Fokus bei der Anwendung der Messmethode auf Strukturen mit wohldefinierten Streufeldern und Geometrien liegt daher bei den FePt- und bei den CoPt-Stukturen. 


\section{Magneto-optische Messmethode zur lokalen magnetischen Abbildung}

Dieses Kapitel soll die applikativen Grundlagen der MOIF-Methode erläutern, die sich den ausgeprägten magneto-optischen Faraday-Effekt (MOFE) in besonderen Materialklassen zu Nutze macht. Eine Optimierung der MOIF-Methode bezüglich Auflösungsvermögen und Sensitivität ist dabei grundlegend für die Abbildung kleiner magnetischer Strukturen, so dass diesen beiden Punkten im Folgenden eine besondere Bedeutung zukommt.

\subsection{Messprinzip zur Abbildung von Streufeldern magnetischer Strukturen}

\section{Magneto-optischer Faraday-Effekt}

Der MOFE beschreibt die Drehung der Polarisationsebene von linear polarisiertem Licht beim Durchgang durch ein optisch aktives Medium, wenn die Lichtausbreitungsrichtung parallel zu einem, das Medium durchsetzenden, externen Magnetfeld oder einer im Medium vorhandenen Magnetisierung ist. Im ersten Fall resultiert diese Aufspaltung aus einer ausgeprägten Zeeman- Aufspaltung der Energieniveaus, im zweiten aus einer unterschiedlichen Besetzung der Spin-Bänder. Ohne detaillierter auf den quantenmechanischen Ursprung des MOFE einzugehen (Details z.B. in [25]), lässt er sich phänomenologisch aus der Phasenverschiebung zwischen links- und rechtszirkular polarisierten Teilwellen des linear polarisierten Lichts infolge unterschiedlicher Dispersionsrelationen erklären. Die daraus resultierenden ungleichen Brechungsindizes $n_{+}$und $n_{-}$für die beiden Teilwellen haben verschiedene Ausbreitungsgeschwindigkeiten $c / n_{+}$bzw. $c / n_{-}$zur Folge, die sich in einer Drehung um den Faraday-Rotationswinkel $\alpha$ der Polarisationsebene des Lichts beim Durchgang durch das aktive Medium manifestieren

$$
\alpha(\omega, l)=\frac{\omega}{2 c}\left[n_{l}(\omega)-n_{r}(\omega)\right] \cdot \Delta z .
$$

$\omega$ stellt die Kreisfrequenz des eingestrahlten Lichts, $c$ die Lichtgeschwindigkeit und $\Delta z$ den zurückgelegten Weg in der Materie dar. Für Gleichung 5.1 wurde ein homogenes Magnetfeld bzw. eine homogene Magnetisierungsausrichtung im Medium vorausgesetzt, so dass die Differenz der Ausbreitungsgeschwindigkeiten über die gesamte Dicke als konstant angesetzt werden kann. Im allgemeinen Fall inhomogener Verteilungen variieren die Brechungsindizes $n_{r, l}(M(z), H(z))$ als Funktion der ortsabhängigen Größen und zur Bestimmung des Gesamtdrehwinkels muss z-abhängig aufintegriert $\alpha_{\text {ges }}=\int d \alpha(M(z))$ werden. 
5. Magneto-optische Messmethode zur lokalen magnetischen Abbildung

Der funktionale Zusammenhang zwischen gemessener Intensität als Funktion des Faradaywinkels wird später dargestellt. An dieser Stelle wichtig ist die Konsequenz, dass die Dicke der aktiven Schicht zum einen für die Gesamtrotation und damit für das gemessene Signal entscheidend ist, aber andererseits auch limitierend wirkt, da lediglich Mittelwerte der Messgröße zugänglich werden.

\section{Magneto-optisches Messprinzip}

Im Wesentlichen gibt es zwei Materialklassen, die einen großen Faraday-Effekt zeigen und sich deshalb zur Streufelddetektion eignen. Das sind zum einen die sogenannten paramagnetischen Salze (Seltenerd-Chalkogenide oder Seltenerd-Halogenide), wie z.B. das paramagnetische EuSe, bei denen, wie oben beschrieben, eine starke optische Anisotropie aufgrund der Zeeman-Aufspaltung der Energieniveaus in einem statischen, externen Magnetfeld auftritt [109]. Diese Klasse der magneto-optischen Sensoren wurde in dieser Arbeit nicht verwendet.

Die weitaus gebräuchlicheren Sensormaterialien sind sogenannte ferrimagnetisch dotierte Eisengranate der Formel $\left\{\mathrm{Me}^{3+}\right\}_{3}\left[\mathrm{Fe}^{3+}\right]_{2}\left(\mathrm{Fe}^{2+}\right)_{3} \mathrm{O}_{12}^{2-}$, die eine spontane Magnetisierung $M_{s}$ aufweisen, dessen Richtung aufgrund verschiedener Anisotropieenergiebeiträge (z.B. magnetokristalline Anisotropie, Wachstumsanisotropie) in der Filmebene liegt [110, 111].

Das grundlegende Messprinzip basiert auf der Ausrichtung dieser Magnetisierung in Richtung der Lichtausbreitung und ist wesentlich abhängig von den spezifischen Sensoreigenschaften, die im nächsten Abschnitt dargestellt werden. Abbildung 5.1(a) zeigt das Messprinzip in einer schematischen Darstellung. Zur Streufelddetektion wird der Sensor mit einer Reflexionsschicht auf die zu untersuchenden Strukturen aufgelegt bzw. vorsichtig angepresst. Die Beleuchtung des Indikator-Ferromagnet-Systems mit polarisiertem Licht erfolgt in senkrechter Inzidenz. Das Licht durchläuft nach Durchgang des Substrats die aktive Sesnorschicht, wird an einer Al-Spiegelschicht reflektiert und passiert die aktive Schicht ein zweites Mal bis es auf den Analysator trifft. Da das Vorzeichen des Winkels $\alpha$ direkt vom Vorzeichen der Magnetisierung im Sensor abhängt, führt dies zu einer Verdopplung des Rotationswinkels und somit zu einer Signal- und Kontrastverstärkung. Da der longitudinale Faraday-Effekt nur auftritt, wenn die Ausbreitungsrichtung parallel zur Sensormagnetisierung ist, tritt eine Drehung der Polarisationsebene bei $M_{z} \neq 0$ auf. Liegt die spontane Magnetisierung, wie in den verwendeten Indikatoren, in der Filmebene, muss sie durch ein externes Feld $\vec{H}_{\text {ext }}=H_{z}$ aus der Ebene herausgedreht werden. Da die z-Komponente der Magnetisierung eine eindeutige Funktion der z-Komponente des äußeren magnetischen Feldes ist, mit $M_{z}=\chi H_{z}$ und $\chi$ der magnetischen Suszeptibilität der aktiven Schicht, resultiert in dieser Messgeometrie eine Sensitivität auf externe $H_{z}$.

Die Drehung des in der Ebene liegenden Magnetisierungsvektors $\vec{M}$ bei einem von außen anliegenden magnetischen Feld $H_{z}$ kann durch den Verkippwinkel $\phi$ beschrieben werden

$$
\phi=\arctan \left(\frac{H_{z}}{H_{a}}\right)
$$

woraus sich $M_{z}=M_{s} \sin \phi$ ergibt. $H_{a}$ steht für das Anisotropiefeld der aktiven Schicht. Aus $M_{z}$ folgt eine dazu proportionale Faradaydrehung $\alpha$ 
(a)

(b)

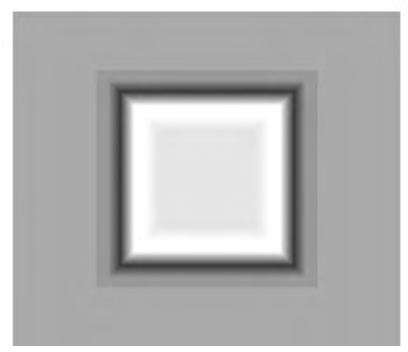

(c)

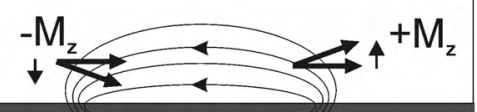

FM $2 \geq$

\section{Substrat}

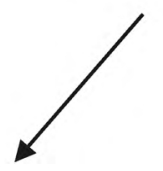


hernd gekreuzte Stellung) beträgt, detektiert man somit eine lokale Intensitätsänderung an Stellen, an denen $H_{z}$ den Sensor durchsetzt, ansonsten erhält man nahezu vollständige Auslöschung. Der entstehende Hell-Dunkel-Kontrast kann von einer CCD-Kamera aufgenommen und die zweidimensionale Intensitätsverteilung $I(x, y)$ z.B. in einer Grauwertdarstellung ausgeben werden. Abbildung 5.1(b) und (c) zeigen schematisch den magnetischen Kontrast, wie er von den Streufeldverteilungen der beiden Strukturen in Abb. 5.1(a) erzeugt wird. Dabei wurde nur der qualitative Verlauf von $H_{z, \text { ext }}$ zugrundegelegt und stellt keine Rechnung oder Messung dar. In Abbildung 5.2 ist zum Vergleich die Messung einer senkrecht aufmagnetisierten FePt-Struktur der Fläche $10 \times 10 \mu m^{2}$ in der typischen Grauwertdarstellung gezeigt.

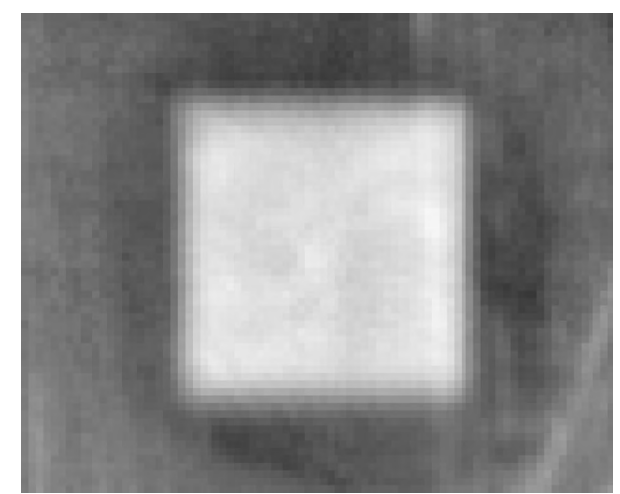

Abbildung 5.2: Magneto-optische Intensitätsverteilung als Grauwertbild von einem $10 \times 10 \mu \mathrm{m}^{2}$ großen, senkrecht aufmagnetisierten FePt-Quadrat.

Quantitativ lässt sich nun die detektierte Intensität nach Durchgang durch den Analysator mit Hilfe des Gesetzes von Malus berechnen

$$
I=I_{1}+I_{0}^{\prime} e^{-2 \gamma d} \sin ^{2}(\alpha \pm \delta)=I_{1}+I_{0} \sin ^{2}(\alpha \pm \delta)
$$

In dieser Form liegt der Gleichung 5.4 bereits die gekreuzte Stellung von Polarisator und Analysator zugrunde. $I_{0}^{\prime}$ entspricht der Ausgangsintensität nach Passieren des Polarisators und vor Eintritt in den Indikator und $\gamma$ dem Absorptionskoeffizienten der magneto-optisch aktiven Schicht der Dicke d. Der Winkel $\delta$ beschreibt die Abweichung der PolarisatorAnalysator- Stellung von der gekreuzten Ausrichtung und hat einen wesentlichen Einfluss auf die Kontrastverbesserung der Abbildungsmethode. Zudem kann so das Vorzeichen von $\alpha$ bzw. $H_{z}$ ermittelt werden. Die additiven Konstante $I_{1}$ berücksichtigt Imperfektionen der optischen Komponenten, wie z.B. den endlichen Polarisationsgrad der Filter, Depolarisationseffekte und von außen eingetragenes Streulicht.

Wie in Abschnitt 5.3 erläutert wird, bilden die Gleichungen 5.3 und 5.4 die Grundlage für eine quantitative Streufeldbestimmung.

\section{Eisengranate als magneto-optische Sensoren}

Eisengranate der Form $\left\{\mathrm{Me}^{3+}\right\}_{3}\left[\mathrm{Fe}^{3+}\right]_{2}\left(\mathrm{Fe}^{2+}\right)_{3} \mathrm{O}_{12}^{2-}$, wobei $\mathrm{Me}^{3+}$ für ein dreiwertiges Metallion steht, zeichnen sich durch ihr ferrimagnetisches Verhalten aus, das aus den un- 
terschiedlich magnetisierten $\left[\mathrm{Fe}^{3+}\right]_{2}$ - und $\left(\mathrm{Fe}^{2+}\right)_{3}$-Untergittern resultiert. Die hohe mikrostrukturelle Qualität der Sensoren ist dabei ebenso wichtig wie eine auf die Verwendung optimierte chemische Zusammensetzung.

Das optisch aktive Material wird mittels Flüssigphasenepitaxie auf einem $500 \mu m$ dicken, transparenten Gadoliniumgalliumgranat-Substrat $\left(\mathrm{Gd}_{3} \mathrm{Ga}_{5} \mathrm{O}_{12}\right)$ abgeschieden [112]. Die entsprechend gewählten Substrate sind gitterangepasst und gewährleisten damit eine bestmögliche Epitaxie. Sie zeigen zusätzlich eine minimale Faraday-Aktivität [110]. Die ebenfalls transparente, aktive Schicht, deren Dicke d im Bereich von typisch $1 \mu \mathrm{m}$ bis $5 \mu \mathrm{m}$ liegt, wird an der Oberseite mit einer 20 - $40 \mathrm{~nm}$ dicken Spiegelschicht aus Aluminium beschichtet. Dieses System aus Substrat, magneto-optisch aktiver Schicht und Spiegel wird im weiteren als sogenannter Sensor oder auch Indikator bezeichnet. $\mathrm{Me}^{3+}$ steht für ein dreiwertiges Seltenerd-Metallion, wobei hier durch das Einbringen verschiedener Substituenten sehr vielfältige Dotierungsmöglichkeiten bestehen. Bringt man diamagnetische, dreiwertige Ionen wie $\mathrm{Y}^{3+}$ oder $\mathrm{Lu}^{3+}$ ein, so verhalten sich die Eisengranate wie beschrieben ferrimagnetisch [113]. Der aus den gegeneinander antiparallel ausgerichteten Untergittermagnetisierungsvektoren verschiedener Größe resultierende Nettomagnetisierungsvektor kann seine Vorzugsorientierung in der Filmebene wie auch senkrecht dazu haben. Die Abbildungscharakteristika von inplane magnetisierten Sensorfilmen sind aber gegenüber den senkrechten stark verbessert.

Tabelle 5.1 zeigt beispielhaft die Zusammensetzung eines in dieser Arbeit verwendten Sensors.

\begin{tabular}{|c||c|c|c|c|c|c|}
\hline Element & $\mathrm{Bi}$ & $\mathrm{Ga}$ & $\mathrm{Lu}$ & $\mathrm{Tm}$ & $\mathrm{Fe}$ & $\mathrm{O}$ \\
\hline Atomverhältnis & 0,9 & 0,56 & 0,42 & 4,41 & 3,4 & 12 \\
\hline
\end{tabular}

Tabelle 5.1: Beispiel für die chemische Zusammensetzung einer in der Arbeit verwendeten aktiven Sensorschicht.

Man erkennt, dass hier eine ganze Reihe von weiteren Seltenerdelementen enthalten sind. Die Erhöhung der Sensitivität der Sensoren durch Herabsetzen der Sättigungsmagnetisierung kann beispielsweise über eine Dotierung mit diamagnetischen Materialien wie Gallium erreicht werden [110]. Das Zulegieren von Bismut steigert die spezifische Faradayrotation [114]. Die Verdet-Konstanten typischer (Bi, Lu, Ga)-Eisengranate liegen zwischen $0,008^{\circ}-0,085^{\circ} \mathrm{mT}^{-1} \mu m^{-1}$ und man erreicht mit solchen Indikatoren eine magnetische Sensitivität bis zu $10 \mu \mathrm{T}[20]$.

\subsection{Experimenteller Messaufbau}

In diesem Kapitel wird der experimentelle Messaufbau beschrieben, der in Abb. 5.3 schematisch dargestellt ist. Die verwendeten optischen Komponenten des Mikroskops sind auf eine möglichst hohe Ausleuchtungsintensität der Probe ausgelegt, sowie auf geringe Depolarisations- und andere Störeffekte. Der Messaufbau ist vor allem in Bezug auf einen optimalen magnetischen Kontrast und eine für diese Methode gute räumliche Auflösung konfiguriert. 


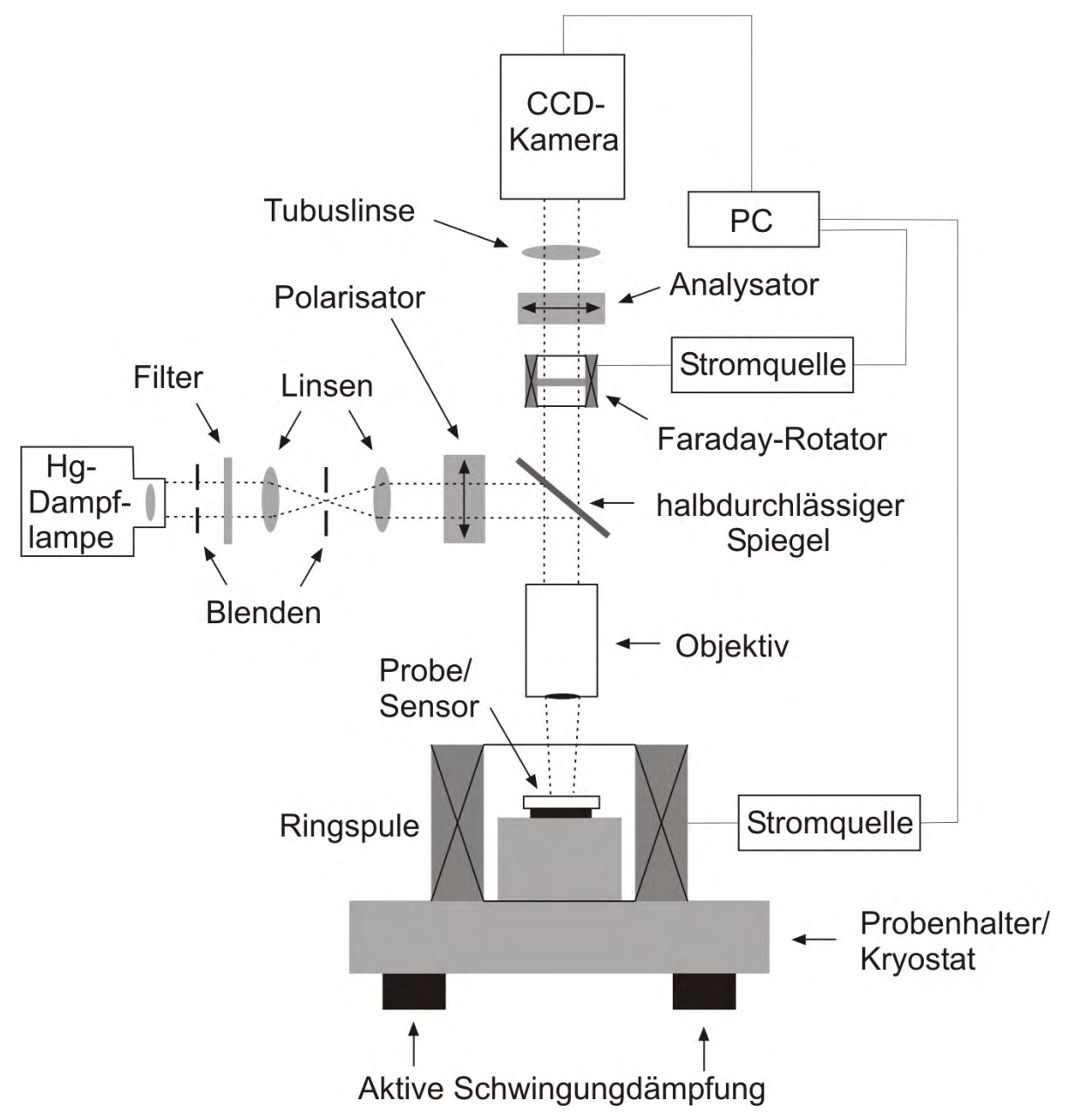

Abbildung 5.3: Messaufbau des magneto-optischen Polarisationslichtmikroskops

Die Probe wird auf dem Probenhalter fixiert, der Sensor mit seiner verspiegelten Seite darauf gelegt und vorsichtig mit in Kleber eingebetteten Edelstahlfüßchen angepresst. Das Anlegen äußerer Magnetfelder geschieht entweder über eine Ringspule, die ein senkrechtes Feld bis zu $330 \mathrm{mT}$ erzeugt oder durch ein Helmholtzspulenpaar für inplane-Felder bis zu $70 \mathrm{mT}$.

Zur Beleuchtung wird eine 200 Watt Quecksilberhochdrucklampe mit integriertem Kollimator verwendet, die bis zu etwa $1 \mathrm{~ms}$ leistungsstabilisiert ist. Somit können Änderungen der detektierten Intensität in der CCD-Kamera aufgrund einer variierenden Ausgangsintensität $I_{0}^{\prime}$ auf langen Zeitskalen vernachlässigt werden. Desweiteren besteht die Beleuchtungseinheit aus zwei Doppellinsen, einer Leuchtfeldblende direkt vor der $\mathrm{Hg}$ Lampe und einer Aperturblende, die gemäß dem Köhlerschen Beleuchtungsprinzip aufgebaut sind. Dieser gewährleistet eine möglichst homogene Ausleuchtung der Probe und die Wahl der Größe des Beleuchtungsfeldes. Die aus Suprasil gefertigten Linsen sind antireflex-beschichtet ( $\lambda / 4$-Beschichtung) um störende Interferenzen und eine Verringerung der Lichtausbeute zu verhindern und zeigen im verwendeten ultravioletten Wellenlängenbereich eine hohe Transmittivität. Ein Wellenlängenfilter begrenzt das Spektrum auf einen Bereich kleiner als $536 \mathrm{~nm}$, da die zur Abbildung verwendeten Sensorfilme bei den entsprechenden Wellenlängen die größte spezifische Faraday-Rotation zeigen. 
Bei Polarisator und Analysator handelt es sich um Glan-Taylor-Prismen, die einen guten Polarisationsgrad bei hoher Transmittivität besitzen $\left(Q=T_{\|} / T_{\perp} \approx 10^{5}\right)$. Die Linsen und die beiden Prismen, sind spannungsarm und zeigen nur eine sehr geringe FaradayAktivität, so dass von der Ringspule erzeugte Magnetfelder am Ort der Komponenten keine störenden Effekte hervorrufen. Der halbdurchlässige Spiegel dient als Strahlteiler und ist zur Vermeidung von Doppelbildern rückseitig ebenfalls entspiegelt. Insgesamt führen die beschriebenen Eigenschaften der optischen Komponenten zu einer starken Reduktion von Depolarisationseffekten bei hoher Ausleuchtungsintensität.

Das Herzstück der im nächsten Kapitel erläuterten Differenztechnik ist der sogenannte Faraday-Rotator. Dieser besteht aus einem Indikator ohne Verspiegelung im Zentrum einer kleinen Kupferringspule durch den, abhängig von der Stärke des äußeren senkrechten Magnetfeldes, ein definierter Drehwinkel $\delta$ eingestellt werden kann. Die Spule des Rotators ist an eine Stromquelle angeschlossen. Die Kennlinie, d.h. die Änderung des Drehwinkels mit der Spannung der Spule, ist in Abbildung 5.4 dargestellt. Ab einem Wert von etwa $3 \mathrm{~V}$ ist der Indikator in Sättigung. Der Maximalwert von $\delta$ liegt bei etwa $1,2^{\circ}$. Dies entspricht auch dem Arbeitswert.

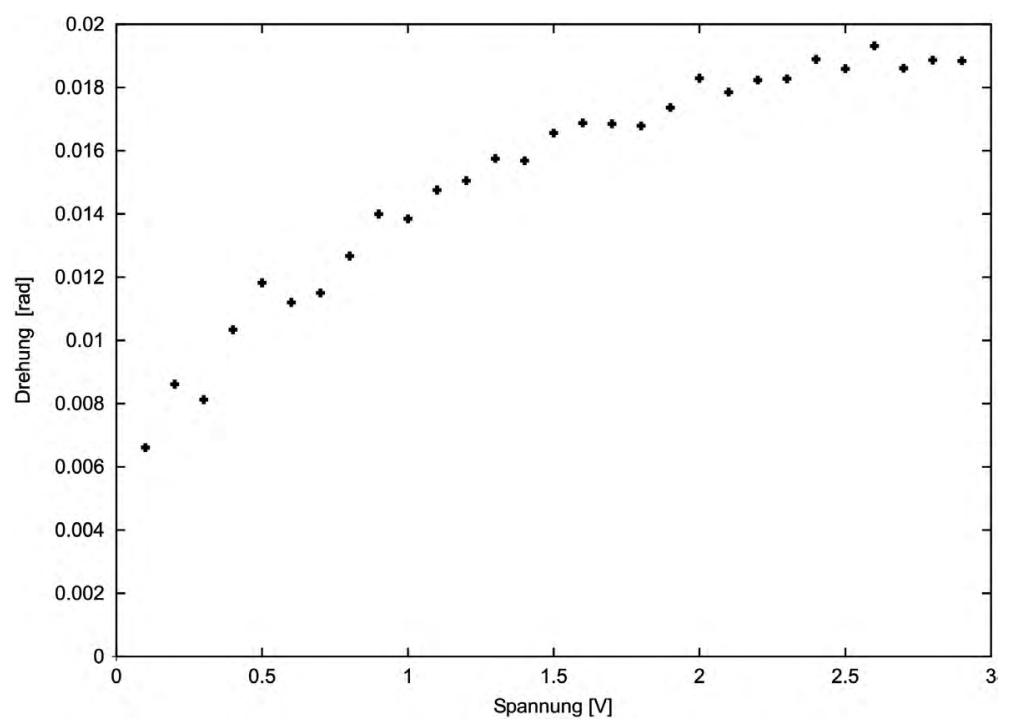

Abbildung 5.4: Faradaydrehung $\delta$ des Rotatorsensors in Abhängigkeit der äusseren Spannung der felderzeugenden Spule. $\delta$ beschreibt in diesem Fall den Verkippwinkel aus der gekreuzten stellung von Polarisator und Analysator heraus.

Die Differenzbildtechnik basiert auf der Subtraktion von zu unterschiedlichen Zeitpunkten aufgenommenen Bildern. Um dies zu gewährleisten, müssen von außen in das System eingetragene, störende Schwingungen gedämpft werden. Dazu ist die gesamte Messapparatur auf einem aktiv schwingungsgedämpften Tisch installiert.

Die Bildaufnahme geschieht über eine CCD-Kamera (LaVision Imager 3), dessen CCDChip eine Auflösung von $1280 \times 1024$ Bildpunkten bei einer Grauwerttiefe von 12 bit besitzt. Zur Reduktion des Dunkelstroms $(<0.1$ Elektronen pro Pixel und Sekunde) wird der Chip durch ein internes Peltier-Element und eine externe Wasserkühlung auf eine Arbeitstemperatur von $-11{ }^{\circ} \mathrm{C}$ gekühlt.

Der Messaufbau erlaubt durch den Einsatz eines Messrechners eine voll automatisierte Datenaufnahme (Software DaVis von LaVision). Insbesondere können die CCD-Kamera und 
5. Magneto-optische Messmethode zur lokalen magnetischen Abbildung

die Stromquellen für die Ringspulen mit definierten Verzögerungszeiten synchron geregelt werden und ermöglicht so die Aufnahme von langen Bildsequenzen mit alternierender Rotatoreinstellung $\pm \delta$.

\subsection{Verbesserung des magnetischen Kontrastes mittels Differenzbildtechnik}

\section{Magneto-optische Differenzbildmethode}

Abbildungsfehler, wie z.B. eine inhomogene Ausleuchtung der Probe oder Fehler im Indikator, lassen sich durch eine einfache Differenzbildtechnik beseitigen, wenn die Probe ohne externes Feld kein eigenes Streufeld produziert (wie z.B. Supraleiter [19]). Bei der Untersuchung von ferromagnetischen Materialien hingegen, die durch ihre spontane Magnetisierung stets ein Streufeld über der zu untersuchenden Struktur produzieren, ist diese Technik nicht mehr so einfach anwendbar.

Ein Differenzbildverfahren, dass auch in solchen Fällen einsetzbar ist, wurde erstmals von Wijngaarden et al. entwickelt [115]. Es basiert auf der gezielten Modulation des Winkels $\delta$ zwischen Polarisator und Analysator aus der gekreuzten Stellung und wird in Anlehnung an die Methode von Wijngaarden auch in dieser Arbeit im Folgenden als sogenannte magneto-optische Lock-In (MOIL)-Technik bezeichnet.

Praktisch wird dieses Verfahren durch den in Kapitel 5.2 beschriebenen Faraday-Rotator realisiert, mit dem man elektronisch definierte Drehwinkel mit unterschiedlichen Vorzeichen $\pm \delta$ einstellen kann. Die Gesamtintensität ergibt sich nach Subtraktion zweier Bilder zu diesen Einstellungen und unter der Näherung, dass sich für kleine Argumente der Sinus linear verhält, gemäß

$$
\begin{aligned}
I_{ \pm} & =I_{+}-I_{-}=\left[I_{1}+I_{0} \sin ^{2}(\alpha+\delta)\right]-\left[I_{1}+I_{0} \sin ^{2}(\alpha-\delta)\right] \\
& =I_{0}\left[(\alpha+\delta)^{2}-(\alpha-\delta)^{2}\right] \\
& =4 I_{0} \alpha \delta .
\end{aligned}
$$

Da $\alpha_{\max } \approx 5-10^{\circ}$ und $\delta=1,2^{\circ}$ ist, ergibt sich aus der Näherung ein Fehler von maximal $1 \%$. Gleichung 5.5 zeigt, dass das Differenzsignal jetzt direkt proportional zum FaradayRotationswinkel und die Proportionalitätskonstante nur noch durch die experimentellen Vorgaben $I_{0}$ und $\delta$ gegeben ist. Für die Sensitivität gegenüber dem Standardverfahren bedeutet dies gerade bei kleinen $\alpha$ eine Verbesserung, da bei der konventionellen Technik $d I / d \alpha$ noch eine Funktion des Drehwinkels selber ist und der nichtlineare Verlauf einen flachen Anfangsbereich zeigt.

Die elektronische Regelung von $\delta$ ist noch aus weiteren Gründen sinnvoll. Bei manueller Einstellung besteht die Gefahr einer Abweichung um $\varepsilon$, also $(-\delta,+\delta+\varepsilon)$. Der Fehler, der aus einer leicht asymmetrischen Modulation der Polarisator-Analysator-Stellung bei der 
Berechnung des Faradaywinkels $\alpha$ aus der experimentell detektierten Gesamtintensität $I_{ \pm}(\varepsilon)$ resultiert, kann mittels Gleichung (5.6) abgeschätzt werden

$$
\begin{aligned}
I_{ \pm}(\varepsilon) & =I_{0}\left[\sin ^{2}(\alpha+(\delta+\varepsilon))-\sin ^{2}(\alpha-\delta)\right] \\
\Rightarrow \quad \alpha(\varepsilon)-\alpha & =\frac{\frac{\varepsilon}{2}+\frac{\varepsilon^{2}}{4 \delta}}{\left(1+\frac{\varepsilon}{2 \delta}\right)} \quad \text { mit } \quad \alpha(\varepsilon)=\frac{I_{ \pm}(\varepsilon)}{4 I_{0} \delta} .
\end{aligned}
$$

Typische manuell eingestellte $\delta=5^{\circ}-10^{\circ}$ und eine Winkeljustagegenauigkeit von $\varepsilon \approx$ $0,5^{\circ}$ resultieren damit in einer Messungenauigkeit für $\alpha_{\max } \approx 5^{\circ}-10^{\circ}$ von $2,5-5 \%$. Bei kleineren Faradaywinkeln kann der Fehler aber deutlich größer ausfallen. Bei elektronischer Regelung ergibt sich aus Abb. 5.4 ein Fehlerintervall für die Einstellgenauigkeit des Rotators von $0,06^{\circ}$, d.h. der Fehler von $\delta$ beträgt maximal $5 \%$. Unter Vernachlässigung des quadratischen Terms in Gleichung 5.6 ergibt sich daraus eine Abweichung in $\alpha$ von $\varepsilon / 2 \approx 0,03^{\circ}$. Dies führt nur bei sehr kleinen Faradaywinkeln $\alpha \leq 1^{\circ}$ zu einem relativen Fehler von $\geq 3 \%$. Zu größeren Drehwinkeln reduziert sich dieser aber auf deutlich kleinere Werte.

\section{Einfluß des Rauschens}

Ein Nachteil dieser Methode besteht in einer Erhöhung des Rauschens, das verschiedene Ursachen haben kann. Die auftretenden Rauschquellen, wie Detektorrauschen (thermisches Dunkelrauschen), die Variation der Ausgangsleistung der Beleuchtungsquelle und das sogenannte Schrotrauschen des Lichts, sind in der Regel statistischer Natur und verstärken sich durch Differenzbildung. Für den Kontrast (Gl. 5.7) hat das bei einem hohen Rauschanteil $\left(I_{ \pm}^{\alpha=0}>>0\right)$ eine deutliche Abweichung vom Maximalwert 1 zur Folge

$$
C \equiv \frac{I_{\max }-I_{\min }}{I_{\max }+I_{\min }}=\frac{I_{ \pm}^{\alpha_{\max }}-I_{ \pm}^{\alpha=0}}{I_{ \pm}^{\alpha_{\max }}+I_{ \pm}^{\alpha=0}}
$$

Wie in Kapitel 5.2 beschrieben, liegt der Dunkelstrom des CCD-Chips nach Kühlung bei $<0,1$ Elektronen pro Sekunde und Pixel. Im Verhältnis zur Gesamtintensität ist dieser Wert im Bereich von 0,1 -1 Promille und verglichen mit den beiden anderen erwähnten Rauschquellen damit vernachlässigbar klein. Hauptursache für die Verstärkung der beiden anderen Rauscharten ist der Einbau des Faraday-Rotators. Der zur Modulation verwendete Rotatorsensor schwächt die am Messindikator auftreffende Intensität ab. Dies führt bei gleichen Belichtungszeiten zu einer schwächeren Gesamtintensität am Detektor. Um diesen Effekt auszugleichen, muss die Belichtungszeit für eine Bildaufnahme auf $\approx 40-90$ ms je nach Messung heraufgesetzt werden.

Das Schrotrauschen ist auf die Quantennatur des Lichts zurückzuführen. Photonen der Energie $E=h \nu$ treffen in zufällig verteilten Zeitabständen auf den CCD-Chip. Dieser stochastische Prozess besitzt eine Poissonverteilung als wirksame Zufallsverteilung, d.h. die in der Kamera detektierten Photonen der Anzahl N können nur noch mit dem Fehler $\delta N=\sqrt{N}$ vorherbestimmt werden. Das Schrotrauschen dominiert vor allem bei sehr kleinen Signalen $(N \ll 1000)$ [116]. Damit wird diese Rauschquelle dominierend gegenüber 
allen anderen. Nach dem Gaußschen Fehlerfortpflanzungsgesetz erhöht sich die durch das Schrotrauschen hervorgerufene absolute Rauschamplitude um den Faktor $\sqrt{2}$. Für das Signal-Rausch-Verhältnis (engl.: signal-to-noise ratio), das den Quotienten aus der absoluten Helligkeitsänderung und der Standardabweichung aller zufälligen Helligkeitsänderungen angibt [117], folgt mit $\delta I_{+}=\sqrt{I_{+}}$bzw. $\delta I_{-}=\sqrt{I_{-}}$(für kleine Winkel)

$$
\mathrm{SNR}=\frac{I_{ \pm}}{\delta I_{ \pm}}=\frac{I_{+}-I_{-}}{\sqrt{\left(\delta I_{+}\right)^{2}+\left(\delta I_{-}\right)^{2}}}=\frac{I_{+}-I_{-}}{\sqrt{I_{+}+I_{-}}}=\frac{2 \alpha \delta}{\frac{I_{1}}{I_{0}}+\left(\alpha^{2}+\delta^{2}\right)}
$$

Man erkennt, dass für ein hohes SNR $I_{1}<<I_{0}$ realisiert werden muss, die Verwendung optischer Komponenten hoher Qualität also sehr wichtig bleibt. Eine Optimierung des Signal-Rausch-Verhältnisses ist gerade bei der optischen Lock-In Technik neben der des Auflösungsvermögens die Grundlage zur Detektion kleiner Messsignale. Dazu muss die Photonenstatistik verbessert werden, was prinzipiell durch eine Erhöhung der Belichtungszeit $(\mu)$ des CCD-Chips der Kamera $(I \propto \mu)$ und/oder einer Aufsummation der Intensitätswerte mehrerer Aufnahmen mit anschließender Signalmittelung realisiert wird. Beide Möglichkeiten sind bei dem verwendeten Messaufbau umsetzbar und sollen im weiteren analysiert werden.

$$
\mathrm{SNR}=\frac{n \cdot I_{ \pm}}{\sqrt{n \cdot I_{ \pm}}}=\sqrt{n} \cdot \frac{I_{ \pm}}{\delta I_{ \pm}} \propto \sqrt{\mu}
$$

Durch Addition von n Aufnahmen mit anschließender Mittelung verbessert sich das theoretische SNR mit $\sqrt{n}$, ebenso wie mit der Wurzel aus der Belichtungszeit.

Abbildung 5.5 zeigt das Signal-Rausch-Verhältnis aufgetragen gegen $\sqrt{n}$ für ein Testobjekt. Dazu wurde die Intensitätsverteilung zweier unterschiedlich ausgerichteter Domänen in einem Indikator, die einen Intensitätssprung von der einen in die andere Domäne zeigen, in Abhängigkeit verschiedener Belichtungszeiten der Einzelbilder ausgewertet. Es wurde sowohl der Sprung, der von den Domänen erzeugten Intensitätsmittelwerte bestimmt, so wie auch deren mittlere quadratische Abweichung (rms-Wert). Das Verhältnis der beiden bestimmten Größen ist als SNR in Abb. 5.5 verwendet worden. Am Beispiel eines Objektives mit 10× nomineller Vergrößerung zeigt sich für alle drei Belichtungszeiten ein nahezu linearer Anstieg des SNR mit $\sqrt{n}$. Weiterhin ist beim Vergleich der Absolutwerte zu den verschiedenen $\mu$ ebenfalls ein Anstieg des $\mathrm{SNR} \approx \sqrt{\mu}$ erkennbar.

Wie erwartet, ergibt sich für einen optimales SNR der Bilder eine möglichst große Anzahl an aufsummierten Differenzbildern als auch hohe Einzelbildintensitäten. Ein wichtiger Punkt, der beachtet werden muss, liegt in der Sättigung der CCD-Kamera. Diese kann aufgrund ihrer 12-bit Datentiefe nur Absolutintensitäten bis zu 4096 cts detektieren. Das maximale Signal in den Einzelbildern darf diesen Wert nicht übersteigen.

\section{Experimentelle Kalibrierung der gemessenen Streufelder}

In den vorhergehenden Abschnitten wurde bereits ausführlich erläutert, wie die aufgenommene Intensitätsverteilung abhängig von dem äußeren Streufeld quantifiziert werden 


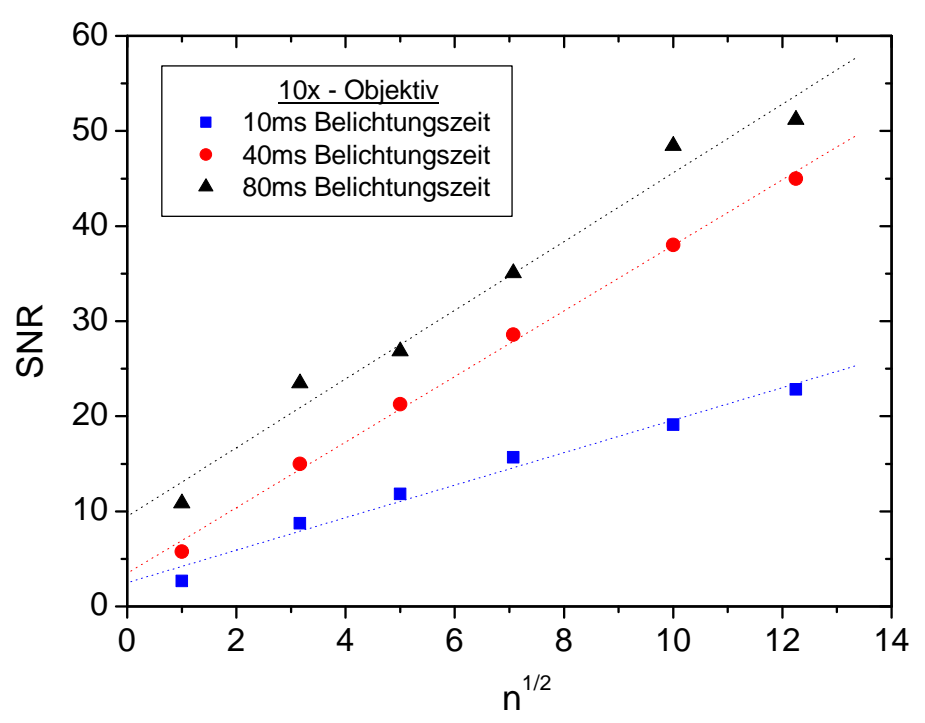

Abbildung 5.5: Signal-Rausch-Verhältnis $(S N R)$ eines Differenzbildes nach Aufsummation und Mittelung über $n$ Differenzbilder aufgetragen gegen $\sqrt{n}$ für verschiedene Belichtungszeiten.

kann. Die Messgröße Intensität soll im Weiteren in das Streufeld selber umgerechnet werden. Der funktionale Zusammenhang zwischen $I_{ \pm}$und $H_{z}$ ergibt sich nach Einsetzen von Gl. 5.3 in Gl. $5.5 \mathrm{zu}$

$$
I_{ \pm}=4 I_{0} \delta c M_{s} \sin \left[\arctan \left(\frac{H_{z}}{H_{k}}\right)\right] .
$$

Zur ortsaufgelösten Bestimmung der senkrechten Komponente des Streufeldes kann man diese Gleichung nun nach $\mathrm{H}_{z}$ umstellen

$$
H_{z}(x, y, I)=H_{k} \tan \left[\arcsin \left(\frac{I_{ \pm}(x, y)}{4 I_{0}(x, y) \delta c M_{s}}\right)\right] .
$$

Die Kalibrierung des Indikators geschieht über eine Ringspule, die ein definiertes, konstantes Magnetfeld senkrecht zur Probe erzeugt. Durch Messung der Intensität in Abhängigkeit dieses Feldes an einer Stelle des Sensors, die nicht mehr von dem Streufeld der zu untersuchenden Proben beeinflusst wird (Außenfeld), kann der Indikator nun über Anpassen der Gleichung 5.11 an die Messdaten $\left(H_{z}, I\right)$ geeicht werden. Mit den ermittelten Parametern ist man in der Lage, jedem Intensitätswert im aufgenommenen Bild einen mittleren $\bar{H}_{z}$-Wert zuzuordnen. In Abbildung 5.6 ist solch eine Prozedur an einem Sensor beispielhaft dargestellt.

Es zeigt sich, dass der Sensor mit einem Sättigungsfeld von $\mu_{0} H_{\text {ext }}=46 \mathrm{mT}$ sehr sensitiv ist und nahezu keine Hysterese aufweist. Dadurch ist der Fehler der Kennlinie bzw. Eichkurve sehr klein. Andere Sensoren können aber auch sehr schwach hysteretisches Verhalten zeigen. Abhängig von dem gewählten Indikator, besonders seiner Sensitivität und der Durchführung der jeweiligen Messungen kann man feststellen, dass der Gesamtfehler nach der Kalibrierung unter Berücksichtigung der oben bereits dargestellten Limitierungen, zwischen etwa 0,5-1 mT liegt. 


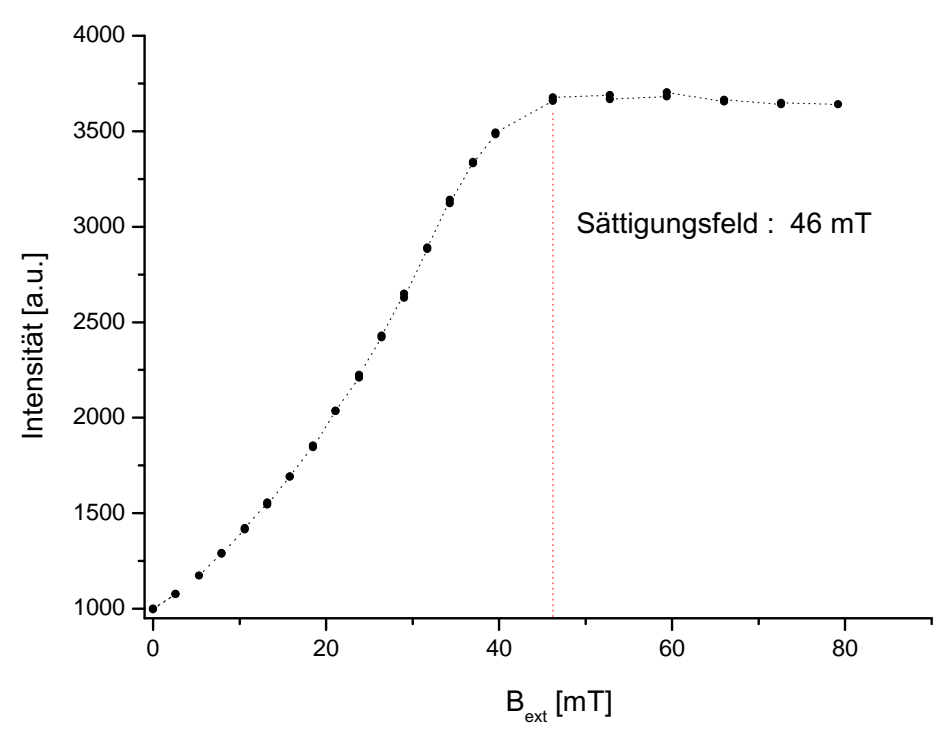

Abbildung 5.6: Kalibrierung des lokalen Streufeldes durch Anpassung der Eichfunktion $H_{z}(x, y, I)$ an die Messdaten.

Diese Kalibrierung liefert nun die Basis für eine voll quantitative, lokale Bestimmung der mittleren senkrechten Komponente des Streufeldes. Die gemessene Intensität ist prinzipiell eine Funktion des, über die Höhe der aktiven Schicht, aufintegrierten FaradayRotationswinkels $I^{\text {Mess }}\left(\int_{\Delta z} d \alpha\left(H_{z}(z)\right)\right)$. Da die Kalibrierung mittels eines konstanten, externen Feldes und damit über einen, innerhalb der aktiven Sensordicke, konstanten Faradaywinkel erfolgt, lassen sich durch die Eichung der Aufnahmen gemäß den Gleichungen 5.10, bzw. 5.11 nur Aussagen über gemittelte Streufeldwerte $\left(\bar{H}_{z}(x, y, \bar{I})\right)$ erzielen

$$
I^{K a l}\left(H_{z, e x t}\right) \stackrel{!}{=} I^{M e s s}\left(\int d \alpha\left(H_{z}(z)\right)\right) \longrightarrow I^{\text {Mess. }}(\bar{\alpha}(z))=I^{\text {Mess. }}\left(\bar{H}_{z}(z)\right)
$$

Zu ergänzen ist, dass bei der Untersuchung von magnetischen Strukturen selbstverständlich beide Magnetfeldrichtungen $\left(+M_{z},-M_{z}\right.$, bzw. $\left.+H_{z},-H_{z}\right)$ auftreten können, so dass der Indikator in beide Feldrichtungen korrekt geeicht werden muss. Hierzu ist es erforderlich auch negative Felder, die eine Reduktion der Gesamtintensität bewirken, von außen anzulegen, um die Eichfunktion auch in diesem Streufeldwertebereich möglichst genau zu bestimmen.

Abbildung 5.7 zeigt eine kalibrierte $H_{z}(x, y)$-Verteilung am Beispiel von FePt-Strukturen verschiedener Kantenlängen $(10 \mu \mathrm{m}, 5 \mu \mathrm{m}, 2 \mu \mathrm{m}$ und $1 \mu \mathrm{m})$, die nach dem oben dargestellten Kalibrierungsverfahren bestimmt wurde. 


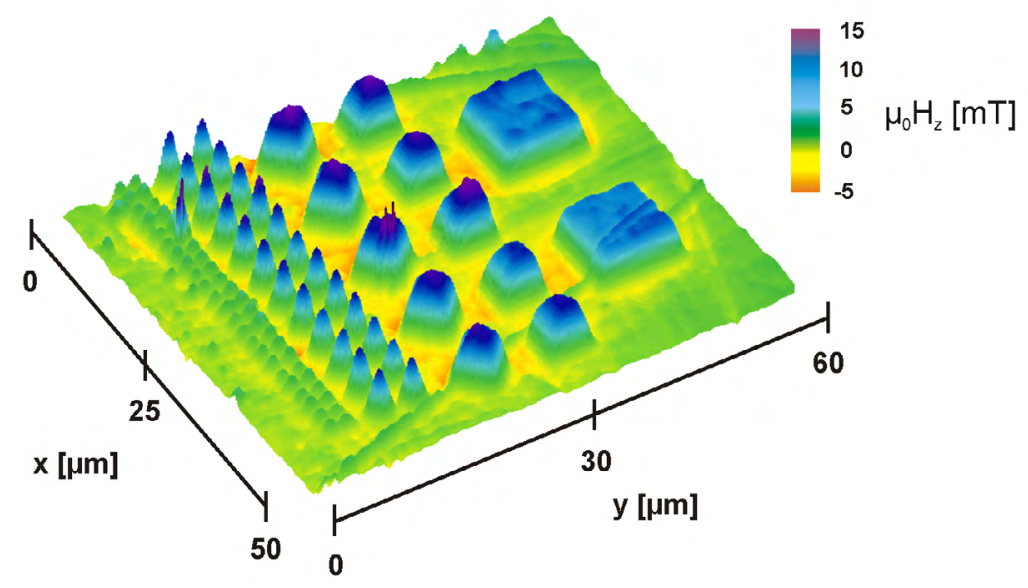

Abbildung 5.7: Kalibrierte Streufeldverteilung von FePt-Strukturen in einer 3D-farbkodierten Darstellung.

\subsection{Optimierung des magnetischen Auflösungsvermögens}

\section{Theoretisches Auflösungsvermögen der MOIF-Methode}

Das theoretische Auflösungsvermögen der MOIF-Methode setzt sich aus den Beiträgen des optischen Abbildungssystems und denen des Sensors zusammen. Nach der klassischen Optik gilt für das Aufösungsvermögen das sogenannte Rayleigh-Kriterium (auch Abbe'sches Beugungslimit) [118]

$$
\Delta x=0,61 \cdot \frac{\lambda}{\mathrm{NA}},
$$

mit $\Delta x$ als der Abstand zwischen zwei benachbarten Objekten, $\lambda$ der Wellenlänge des zur Abbildung verwendeten Lichts und NA der numerischen Apertur des Abbildungssystems. Danach ergeben sich für die verwendeten Objektive die in Tabelle 5.2 zusammengefassten Werte.

\begin{tabular}{|c||c|c|c|}
\hline nominelle Vergrößerung & $10 \times$ & $20 \times$ & $50 \times$ \\
\hline numerische Apertur NA & 0,30 & 0,50 & 0,55 \\
\hline optische Auflösung $\Delta x[\mathrm{~nm}]$ & 1080 & 650 & 590 \\
\hline
\end{tabular}

Tabelle 5.2: Parameter zur Berechnung der optischen Auflösung aus dem Rayleigh-Kriterium für die in dieser Arbeit verwendeten Objektive angegebener nomineller Vergrößerung. Zur Berechnung wurde eine Wellenlänge von $\lambda=530 \mathrm{~nm}$ angesetzt.

Eine prinzipielle untere Grenze für den Arbeitsabstand ist durch die Dicke der Indikatoren gegeben. Aus diesem Grund besitzt das 50×-Objektiv einen größeren Arbeitsabstand als die beiden mit kleinerer nomineller Vergrößerung, was zu der nur unproportional geringen Zunahme der numerischen Apertur führt. 
5. Magneto-optische Messmethode zur lokalen magnetischen Abbildung

Das Auflösungsvermögen der Sensoren ist abhängig von den charakteristischen, magnetischen Eigenschaften der verwendeten Sensoren und im Wesentlichen von der minimalen Domänenwandbreite $\mathrm{d}_{D W}$, die damit auch ein Maß für die Kopplungsgrößen im Sensor ist. Nach [110] liegt diese bei den dort hergestellten Sensoren für in der Ebene magnetisierte Schichten typischerweise bei $\mathrm{d} \approx 200 \mathrm{~nm}$. Zur Berechnung der theoretischen räumlichen Auflösung für die Streufelddetektion muss man beide dargestellten Beiträge berücksichtigen. Beschreibt man die beiden Auflösungsfunktionen mittels normierter Gaußkurven mit den entsprechenden Varianzen, so ergibt sich für die Auflösung mit $\Delta x_{10 \times}=1110$ $\mathrm{nm}, \Delta x_{20 \times}=670 \mathrm{~nm}$ und $\Delta x_{50 \times}=605 \mathrm{~nm}$ nur eine schwache Verschlechterung des Aufösungsvermögens.

\section{Experimentelles Auflösungsvermögen und Einfluss der aktiven Sensorschichtdicke}

Für das experimentell tatsächlich erreichbare Aufösungsvermögen müssen verschiedene zusätzliche Faktoren berücksichtigt werden. Die Messhöhe spielt dabei eine maßgebliche Rolle, da die Streufelder kleiner magnetischer Strukturen über der Oberfläche sehr stark abfallen. Die untere Grenze für die Messhöhe stellt die Dicke der Aluminium-Spiegelschicht und eventuell vorhandene Rauigkeiten der beteiligten Grenzflächen zwischen Sensor und zu untersuchender Struktur dar. Die Dicke der Spiegelschicht beträgt $d_{\text {Spiegel }}=40 \mathrm{~nm}$ und gewährleistet damit eine gute Reflektivität bei möglichst geringer Dicke. Zum Schutz dieser Schicht ist auf den Sensoren in der Regel ein 10-20 nm dicker, transparenter $\mathrm{TiO}_{2}{ }^{-}$ Film zusätzlich aufgebracht. Wie AFM-Messungen zeigen, beträgt die rms-Rauigkeit der Sensoroberfläche nur $\sigma \approx 1,4 \mathrm{~nm}$ und es gibt keine Anzeichen für eine langwellige Rauigkeitsmodulation. Bei Abwesenheit grober Verschmutzungen, die durch eine Reinigung mit Waschbenzin verhindert werden kann, sollte die Mindesthöhe bei etwa $d_{\text {Spiegel }}=60$ nm liegen.

Da der Faraday-Rotationswinkel mit der Länge des optischen Weges im Sensor zunimmt, zeigt sich eine starke Abhängigkeit des Auflösungsvermögens mit der aktiven Sensordicke. Nach [119] ist das Rayleigh-Kriterium äquivalent zu der Forderung, dass zwei Objekte noch als gerade voneinander trennbar gelten, wenn die Intensität in der zentralen Einsenkung zwischen den Beugungsfiguren 0,735× der Intensität der benachbarten Maxima ist. So formuliert, bietet das Kriterium die Möglichkeit, das Aufösungsvermögen experimentell in Abhängigkeit von der aktiven Schichtdicke abzuschätzen. Dazu wurde das von quadratischen CoPt-Strukturen unterschiedlicher Kantenlänge und Abstand erzeugte Streufeld mit einem Sensor abgebildet, dessen aktive Schichtdicke sukzessiv durch Ionenstrahlsputtern reduziert wurde. Die Anfangsdicke betrug ca. $4 \mu \mathrm{m}$.

Abb. 5.8 zeigt, wie mit abnehmendem Abtrag sich immer feinere Strukturen und kleinere Abstände auflösen lassen (Vergleich weiße Kästchen). Die resultierende, zunehmende Verbesserung des Auflösungsvermögens lässt sich im Folgenden anhand der Intensitätsprofile (vgl. Abb. 5.8 untere Bildreihe) abschätzen. Dazu wurden bei der jeweiligen Dicke der optischen Schicht solche Strukturgrößen ausgewählt, die in der Abbildung klar voneinander separiert erscheinen und die zugeordneten Profile zweier benachbarter Strukturen so verschoben, dass sie dem Rayleigh-Kriterium $\left(I=0,735 \cot I_{\max }\right)$ genügen. 


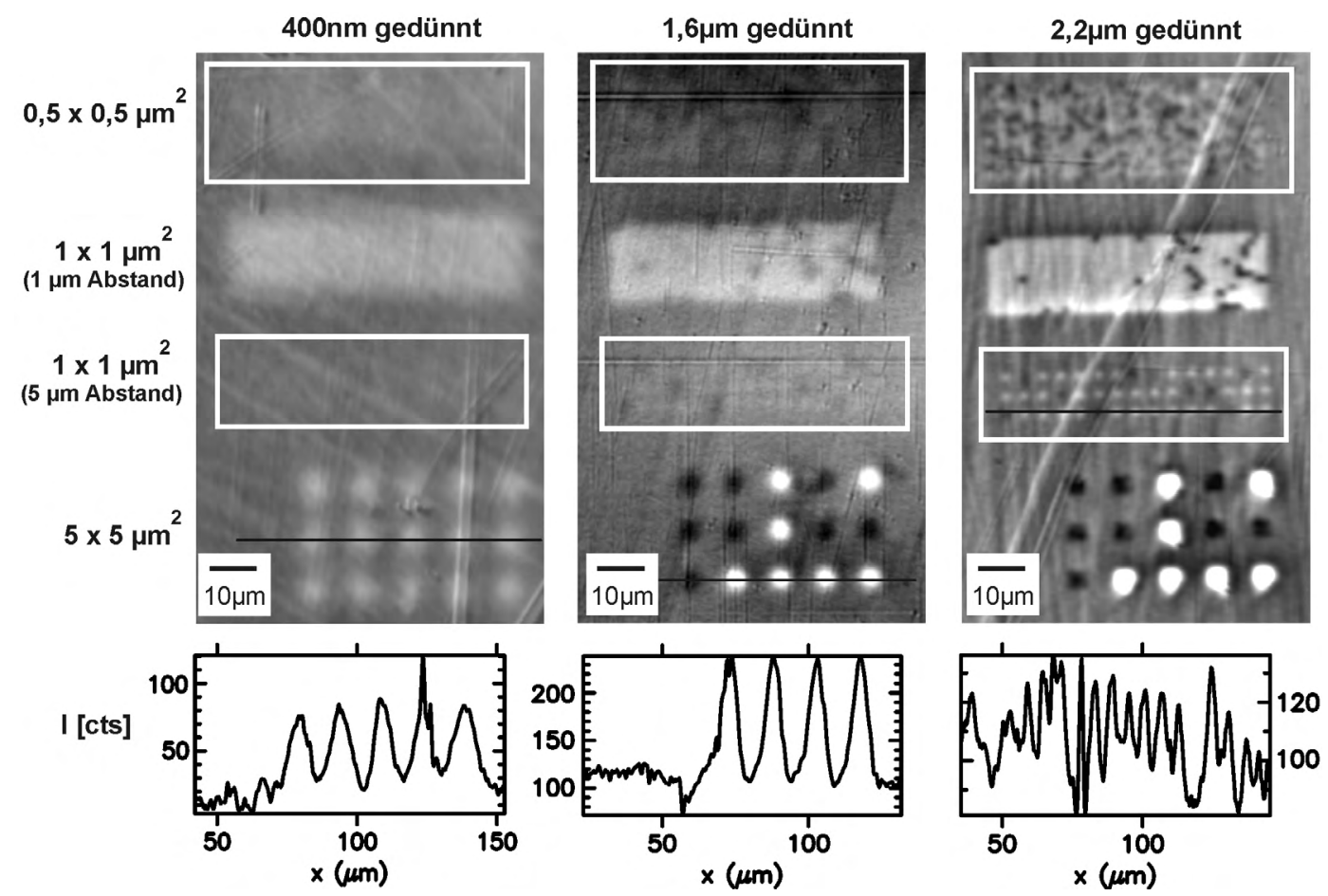

Abbildung 5.8: Intensitätsverteilungen von CoPt-Strukturen bei unterschiedlicher aktiver Sensorschichtdicke. Die Auflösungsverbesserung wurde durch Reduktion der über den Bildern angegebenen Werte für die Schichtdicke erreicht. Die Strukturgrößen und Abstände sind am linken Rand angegeben. Die Intensitätsprofile zeigen den linearen Schnitt durch ausgewählte Strukturen. In Bild 2 und 3 wurden die Strukturen einmal einem Feld von $H_{z, e x t}=300 \mathrm{mT}$ ausgesetzt, nachdem sie in Bild 1 bei $H_{z, e x t}=-1,5 \mathrm{~T}$ aufmagnetisiert worden waren.

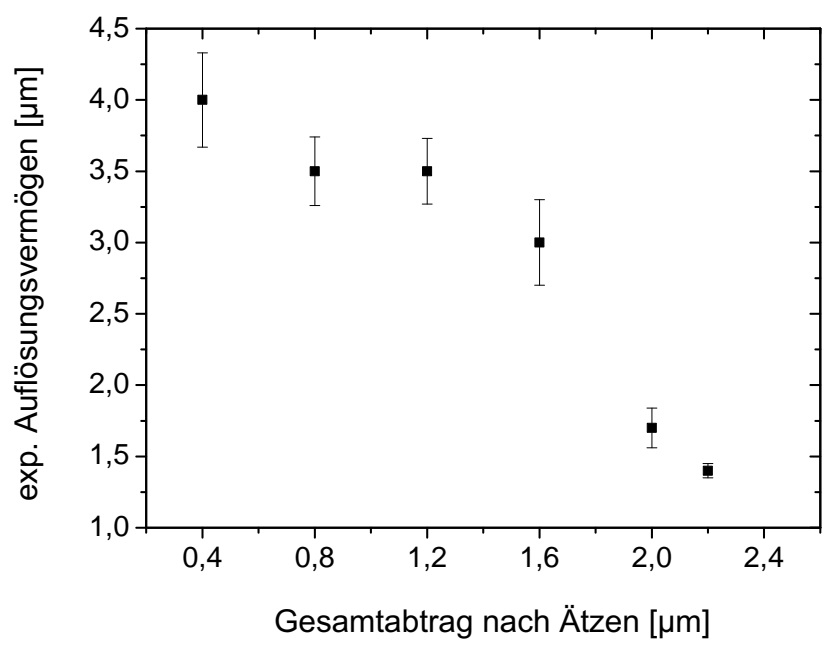

Abbildung 5.9: Mittels Rayleigh-Kriterium abgeschätztes experimentelles, Auflösungsvermögen für den gedünnten Indikator. Als x-Achse ist der Gesamtabtrag dargestellt, da die Ausgangsdicke der aktiven Schicht nicht exakt bekannt ist.

Abbildung 5.9 zeigt die so abgeschätzten Auflösungsvermögen in Abhängigkeit vom Sensorabtrag. Eine magnetische Auflösung im Bereich von $1 \mu \mathrm{m}$ erfordert demnach sehr dünne 
5. Magneto-optische Messmethode zur lokalen magnetischen Abbildung

optisch aktive Schichten. Besonders deutlich wird dies in Abbildung 5.10. Hier wurde ein Sensor, der nur eine aktive Schichtdicke von $d=600 \mathrm{~nm}$ hat, verwendet. Hier ergibt sich die experimentelle Auflösung mit $800( \pm 50) \mathrm{nm}$ sehr nahe am theoretischen Limit der abbildenden Optik.
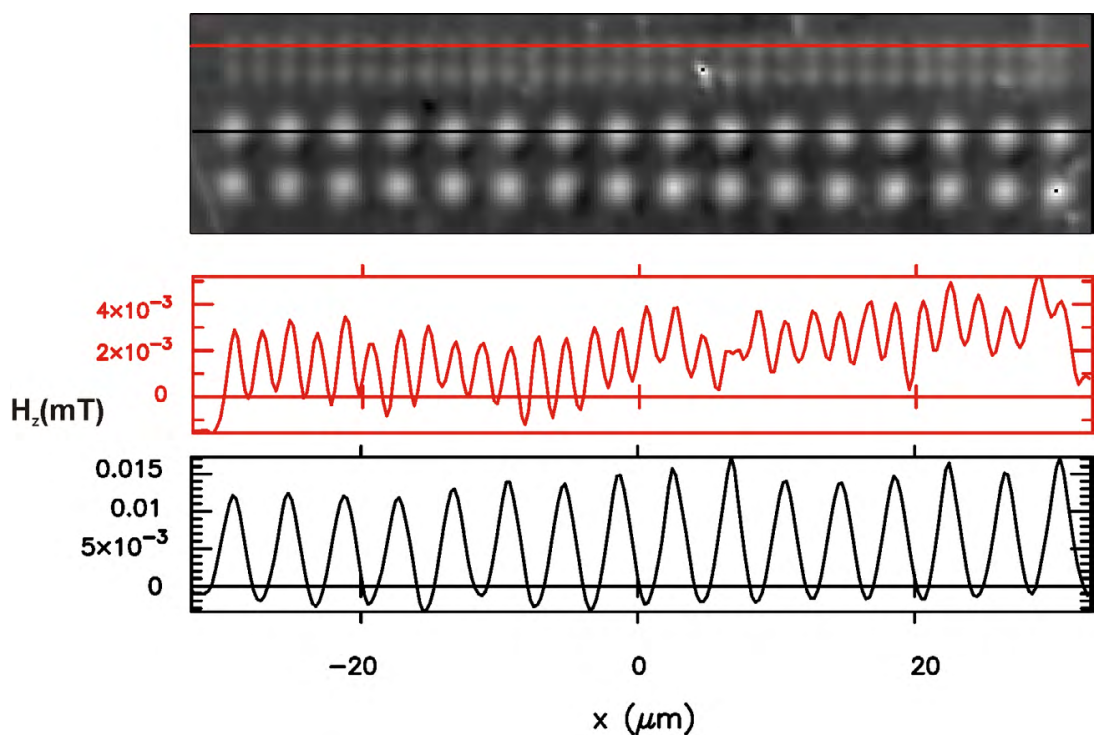

Abbildung 5.10: Hochaufgelöste magneto-optische Aufnahme der kalibrierten Streufeldverteilung von FePt-Strukturen mit einem $600 \mathrm{~nm}$ dünnen Sensor. Abgebildet sind Strukturen mit $1 \mu \mathrm{m}$ Kantenlänge und $1 \mu \mathrm{m}$ Abstand (oberen beiden Reihen) und $2 \mu \mathrm{m}$ Kantenlänge mit $2 \mu \mathrm{m}$ Abstand (unteren beiden Reihen). Die experimentelle Auflösung ergibt sich aus den Profilen zu etwa $800 \mathrm{~nm}$.

\subsection{Simulation von Streufeldern bei rein senkrechter Magnetisierung}

Nach der experimentellen Quantifizierung der $\mathrm{H}_{z}$-Komponente des magnetischen Streufeldes in Kapitel 5.3 soll in diesem Abschnitt ein Vergleich mit berechneten Streufeldverläufen rechteckiger Proben dargestellt werden. Ziel ist es, den Einfluss der intrinsischen Messbedingungen, wie endliche Sensordicke und begrenztes magnetisches Auflösungsvermögen, auf die Streufeldbestimmung detaillierter zu verstehen.

Das Biot-Savart'sche-Gesetz (Gl. 2.7) bildet den Ausgangspunkt für die Berechnung der Streufelder einer zunächst beliebig dimensionierten, rechteckigen Probe mit homogener, senkrechter Magnetisierung

$$
\vec{B}(\vec{r})=\frac{\mu_{0}}{4 \pi} \int_{V} \vec{\jmath}_{M}\left(\vec{r}^{\prime}\right) \times \frac{\vec{r}-\vec{r}^{\prime}}{\left|\vec{r}-\vec{r}^{\prime}\right|^{3}} d^{3} r^{\prime}
$$

Abbildung 5.11(a) verdeutlicht schematisch die Modellierung der Magnetisierung mittels einer magnetischen Oberflächenstromdichte $\vec{\jmath}_{M}$ für eine quaderförmige Probe und 5.11(b) die im Weiteren gewählte Nomenklatur. 
a.)

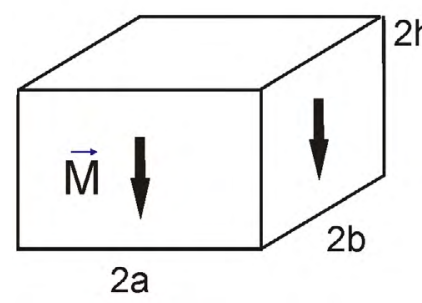

b.)

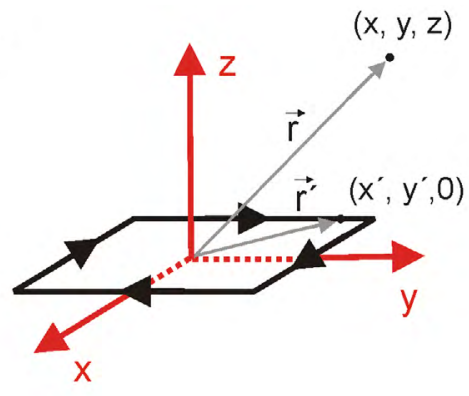

Abbildung 5.11: (a) Schematische Darstellung der homogenen, senkrechten Magnetisierung eines Körpers über idealisierte Oberflächenströme als Grundlage der Berechnung seines Streufeldes mittels Biot-Savart'schen Gesetzes. (b) Skizze zur Festlegung der Nomenklatur.

Die Lösung des Integrals ergibt sich mit der Remanenzpolarisation $P_{r}$ zu (Herleitung und Darstellung der zurücksubstituierten Gleichung siehe Anhang)

$$
B_{z}(\vec{r})=-\frac{P_{r}}{4 \pi}\left[\left[\left[\arctan \left(\frac{\gamma \sqrt{\alpha^{2}+\beta^{2}+\gamma^{2}}}{\alpha \beta}\right)\right]_{\alpha_{1}}^{\alpha_{2}}\right]_{\beta_{1}}^{\beta_{2}}\right]_{\gamma_{1}}^{\gamma_{2}} .
$$

Abbildung 5.12 zeigt nun den Vergleich zwischen der so simulierten z-Komponente des Streufeldes (a) für eine $10 \mu m \times 10 \mu m$ große FePt-Struktur mit der MOIF-Messung (b) an einem solchen Quadrat. Die Magnetisierung zeigt dabei aus der Bildebene heraus. Für den Simulationsparameter remanente Polarisation $P_{r}$ wurde hierbei der durch SQUIDMessungen experimentell bestimmte Wert der Sättigungspolarisation $P_{s}=1,42 \mathrm{~T}$ der FePt-Schicht (siehe Abb. 4.6) verwendet. Da die Remanenz des unstrukturierten Films nur einen Wert von $P_{r}=1,31 \mathrm{~T}$ zeigt, ist mittels magnetischer Kraftmikroskopie die Magnetisierungskonfiguration dieser FePt-Struktur abgebildet worden. Abb. 5.12(c) bestätigt den eindomänigen Zustand, so dass die Simulation mit dem Sättigungswert der Polarisation durchgeführt werden kann. Das simulierte Feld wurde in einem Abstand von $z=140$ $\mathrm{nm}$ von der Oberfläche bestimmt, also in einem Abstand der vergleichbar der Mindesthöhe einer magneto-optischen Abbildung ist. Die Simulation ergibt eine Verteilung, die charakteristisch für das Streufeld einer Oberflächenstromdichte für senkrecht aufmagnetisierte Strukturen ist. Der physikalische Rand der Struktur fällt mit den Nulldurchgängen des Streufeldes zusammen. Im Innenraum, sehr nahe des Randes, erkennt man gut die starken Felderhöhungen, die ihren Ursprung in dem an den Rändern lokalisierten, effektiven Magnetisierungsstrom haben (Vergleich Abb. 5.11). Zum Mittelpunkt der Struktur hin fällt das $H_{z}$ schnell ab und bleibt dann nahezu konstant. Da die Feldlinien geschlossen sind, kehrt sich die Feldrichtung im Außenraum um.

Der Vergleich mit der experimentell gemessenen Struktur zeigt deutlich, dass sich die beiden Feldverläufe unterscheiden. Besonders die berechneten Feldwerte in der Nähe des Randes sind viel zu hoch. In der Mitte der Struktur ergibt sich dagegen schon eine durchaus gute Übereinstimmung. Der Unterschied in den Verteilungen lässt sich physikalisch recht einfach verstehen, da in der Simulation lediglich eine feste Messhöhe zur Berechnung des Streufeldes herangezogen wurde. Die starken Abweichungen sind ein klares Indiz dafür, 
(a)
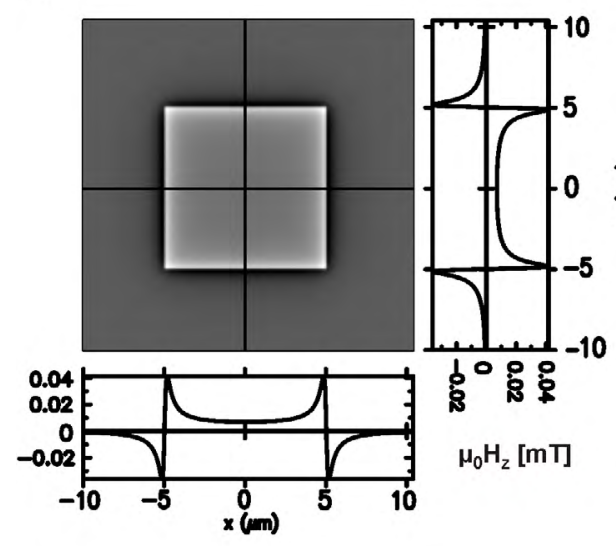

(b)

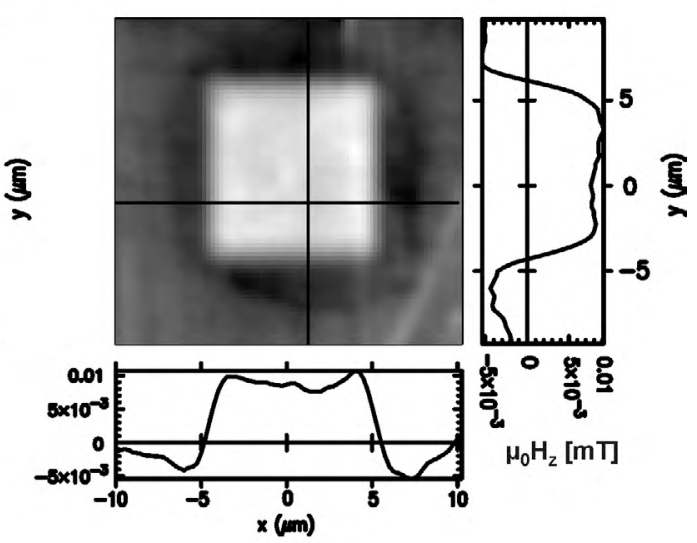

(c)

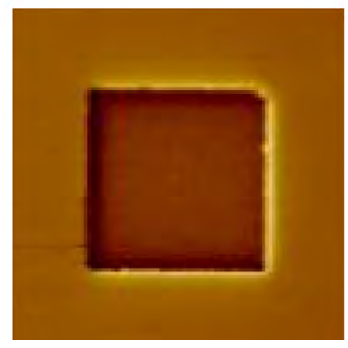

Abbildung 5.12: (a) Simulation der z-Komponente des Streufeldes im Aussenraum eines $10 \mu \mathrm{m} \times 10 \mu \mathrm{m}$ Quadrates mit senkrechter Magnetisierung $P_{s}=\mu_{0} M_{z}=1,41 \mathrm{~T}$ bei fester Messhöhe $z=140 \mathrm{~nm}$. (b) Experimentell bestimmte Streufeldverteilung mittels MOIF-Methode eines FePt-Quadrates gleicher Größe und (c) MFM-Messung zur Charakterisierung der zugrundeliegenden Magnetisierungskonfiguration. Die leicht asymmetrische Intensitätsverteilung in Bezug auf die linke Kante kommt durch eine geringe Neigung der MFM-Spitze um $10^{\circ}$ zustande, um bei der Cantileverschwingung die Spitze nicht auf die Probenoberfläche aufzusetzen.

dass die endliche Sensorschichtdicke bei der magnetischen Abbildung zu einer Mittelung über diese endliche Messhöhe führt, die in der Simulation berücksichtigt werden muss. Ein weiterer wichtiger Punkt, der vor allem bei der quantitativen Abbildung von kleinen Strukturen $(<5 \mu m)$ stark an Gewicht erhält, ist das begrenzte Auflösungsvermögen der Messmethode (siehe vorheriger Abschnitt).

Die beiden genannten Korrekturen sind in Abbildung 5.13 in die Simulation eingearbeitet worden. Die endliche Sensorschichtdicke von $\mathrm{d}_{M O L}=600 \mathrm{~nm}$ ist durch numerische Integration von $\mathrm{H}_{z}(z)$ und Mittelung gemäß

$$
\bar{H}_{z}(x, y)=\frac{1}{d_{M O L}} \int_{d_{0}}^{d_{0}+d_{M O L}} H_{z}(x, y, z) d z
$$

berücksichtigt, wobei für Abb. 5.13 von $\mathrm{d}_{0}=100 \mathrm{~nm}$ bis $\mathrm{d}_{0}+\mathrm{d}_{\text {Sensor }}=700 \mathrm{~nm}$ integriert wurde. In dieser Mittelung wurde vorausgesetzt, dass die Magnetisierung des Indikators unmittelbar dem äußeren Feld folgt. Der sich daraus ergebende messhöhengemittelte Verlauf wurde danach noch mit einer Gaußverteilung (Gl. 5.16) der Varianz $\sigma=800 \mathrm{~nm}$ 


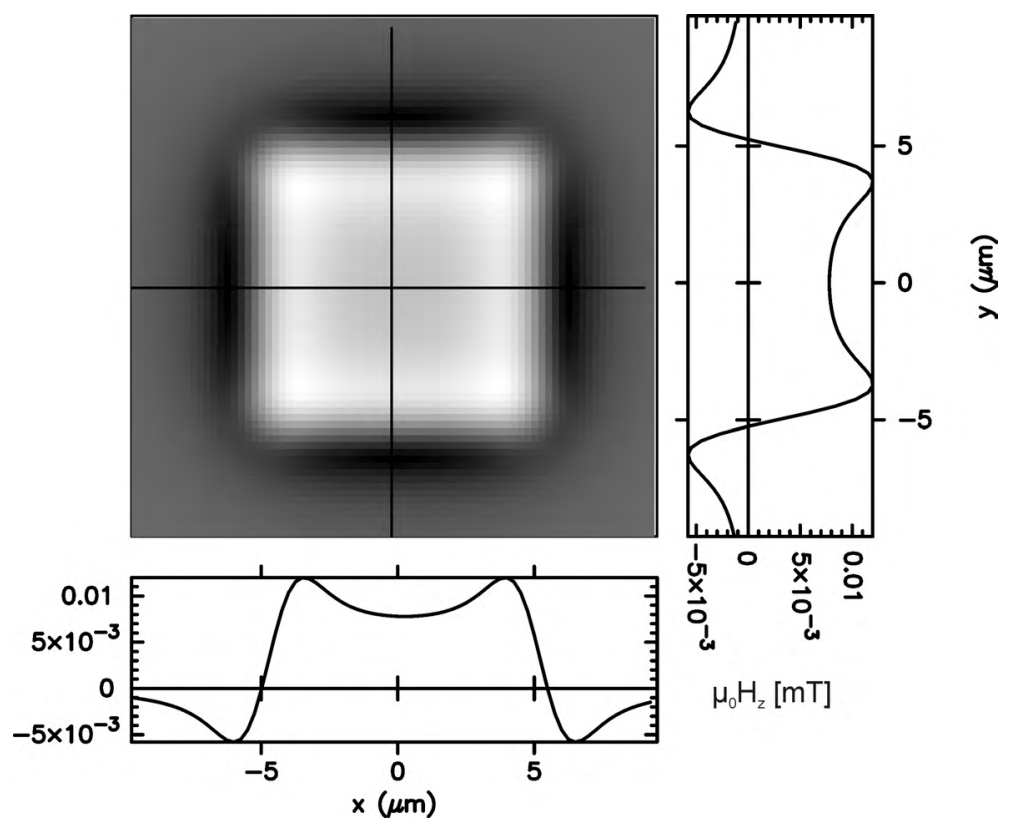

Abbildung 5.13: Theoretische Berechnung des Streufeldes einer $10 \mu m \times 10 \mu m$ - Struktur unter Einbeziehung der endlichen Sensordicke, sowie der experimentell begrenzten Auflösung.

gefaltet, um der begrenzten Aufösung Rechnung zu tragen (vgl. Kap. 5.4). Die numerischen Integrationsroutinen zur Anpassung der letztgenannten Korrekturen wurden von Herrn Norpoth programmiert [43, 39].

$$
G_{\sigma}(x, y)=\frac{1}{2 \pi \sigma^{2}} e^{-\frac{x^{2}+y^{2}}{2 \sigma^{2}}}
$$

Die Berücksichtigung der realen Messbedingungen bei den Berechnungen führt zu einer sehr guten Übereinstimmung bezüglich des charakteristischen Feldverlaufs als auch der quantitativen Feldwerte. Vor allem die Felderhöhungen an den Rändern der Struktur sind nun deutlich reduziert und stellen ein zufriedenstellendes Abbild der experimentellen Daten dar.

Die Tatsache, dass der theoretisch berechnete Streufeldverlauf für eine $10 \mu \mathrm{m} \times 10 \mu \mathrm{m}$ große Struktur sehr gut mit den experimentellen Daten übereinstimmt, bedingt allerdings noch keine wirkliche Vorhersagekraft auch für andere Strukturgrößen. Vor allem für kleinere Abmessungen muss überprüft werden, wie allgemeingültig die Simulationsparameter Auflösungsververmögen und Dickenabhängigkeit sind und ob die Kalibrierung des Sensors zu kleinen Strukturen nicht versagt.

In Abbildung 5.14 ist der Vergleich zwischen den Messungen an kleineren Strukturen mit Simulationen der gleichen Parameter wie oben (rechte Spalte) zu sehen. Um den Einfluss von sich überlagernden Streufeldern bei begrenzter magnetischer Auflösung mit zu berücksichtigen, wurden ebenfalls Anordnungen von Strukturen berechnet. Selbst bei der kleinsten betrachteten Strukturgröße von $1 \mu \mathrm{m}$ ist die Übereinstimmung noch sehr gut, man erkennt lediglich bei den kleinsten Quadraten, dass die Strukturen in der Simulation nicht so klar voneinander separiert sind wie im Experiment. Dies deutet darauf hin, dass die Varianz von $\sigma=800 \mathrm{~nm}$ eher noch zu groß abgeschätzt wurde. Verringert man diesen 
a)
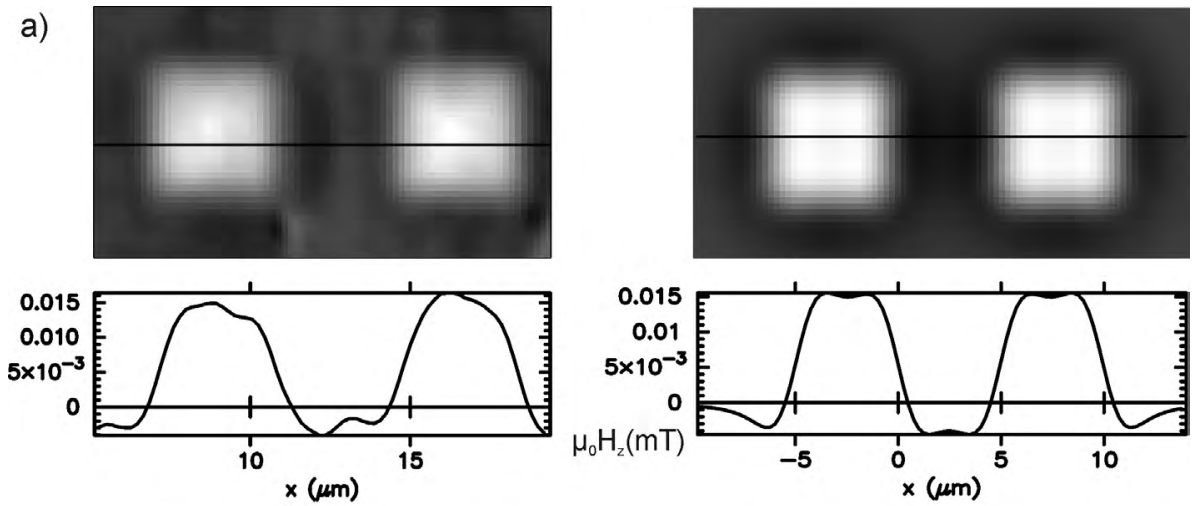

b)
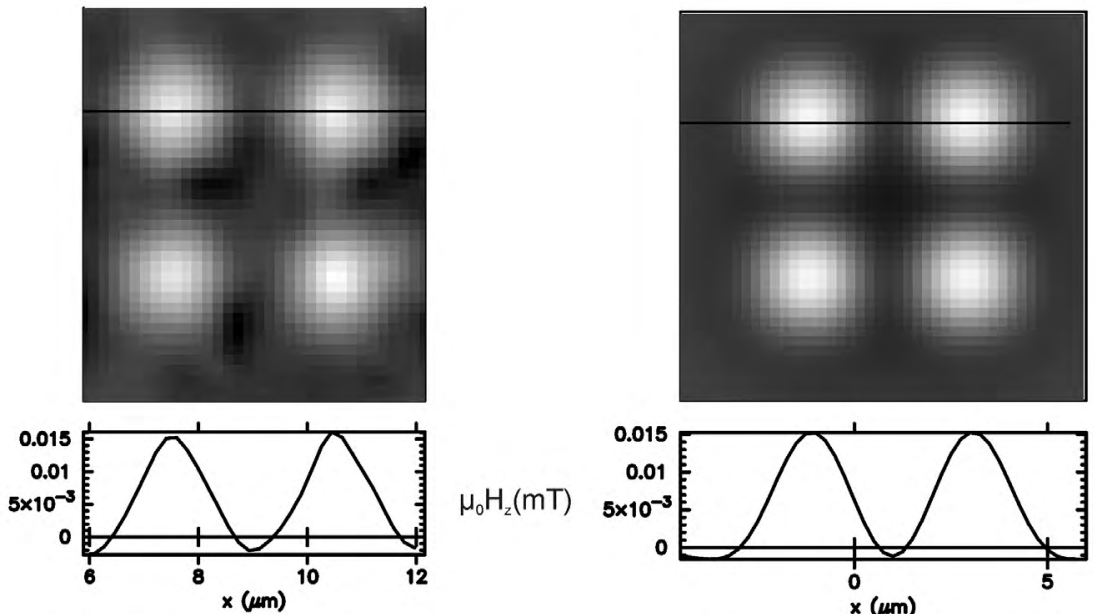

$\mu_{0} H_{2}(m T)$

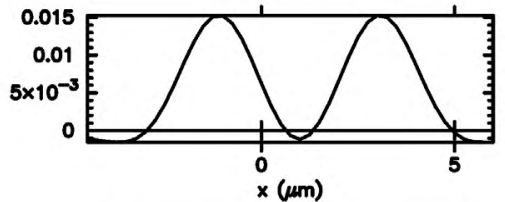

c)
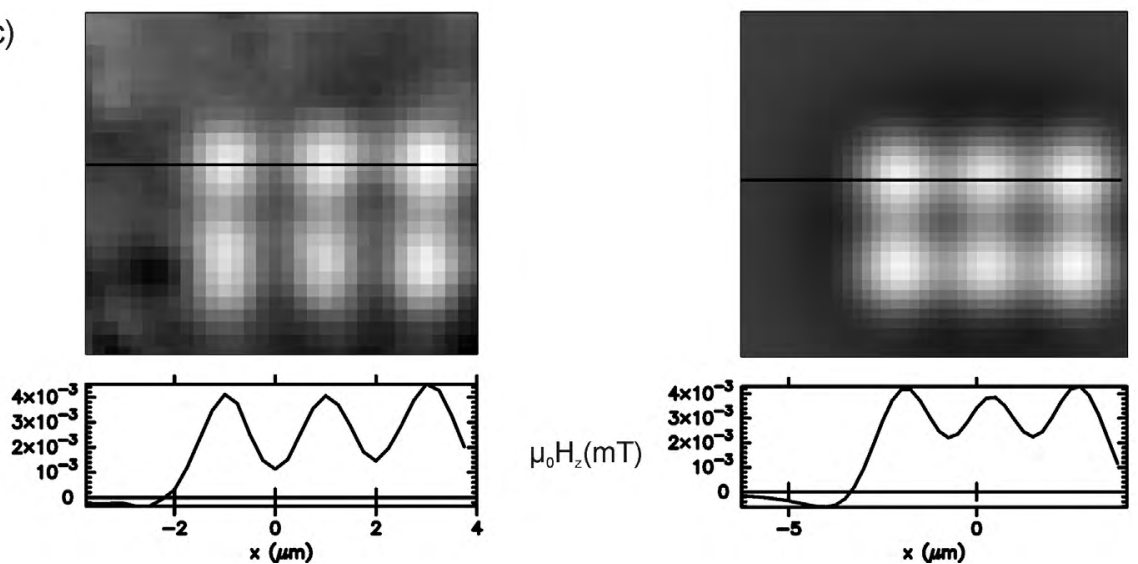

$\mu_{0} H_{z}(m T)$

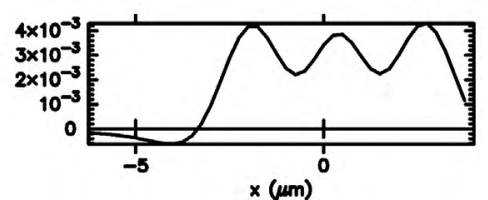

Abbildung 5.14: Vergleich von experimentellen (linke Spalte) und theoretisch berechneten Streufeldverteilungen (rechte Spalte) für Quadrate mit unterschiedlichen Kantenlängen und einer Anordnung in Feldern. Die Simulationen wurden analog Abb. 5.13 unter Einbeziehung der experimentellen Messbedingungen durchgeführt. Die Kantenlängen der Einzelquadrate betragen in (a) $5 \mu m$, (b) $2 \mu m$ und (c) $1 \mu m$.

Wert, so werden jedoch die Werte der Streufelder wieder größer. Da sich Abweichungen bei der exakten Strukturgröße und dem Strukturabstand von 50 - $100 \mathrm{~nm}$ bereits deutlich in den Feldverteilungen auswirken und außerdem ein Fehler von $\approx 1 \mathrm{mT}$ aus der Kalibrierung resultiert, ist diese geringe Abweichung von Experiment und Simulation nicht verwunderlich. 
Der Vergleich zeigt also hier deutlich, dass selbst bei kleinen Strukturgrößen nahe der Auflösungsgrenze das Streufeld quantitativ bestimmt werden kann, wenn man die apparative Auflösung und die endliche aktive Sensordicke berücksichtigt. Letzteres impliziert, dass die Sensormagnetisierung dem äußeren Feld unmittelbar folgt, d.h. in erster Näherung gilt

$$
\bar{H}_{z}(x, y)=\frac{1}{d_{M O L}} \int_{d_{0}}^{d_{0}+d_{M O L}} H_{z}(x, y, z) d z=H_{k} \tan \left[\arcsin \left(\frac{\bar{I}_{ \pm}(x, y)}{4 I_{0}(x, y) \delta c M_{s}}\right)\right]
$$

\subsection{Simulation von Streufeldern bei reiner inplane Magnetisierung}

Die Feldberechnung für rein in der Ebene magnetisierte Strukturen erfolgt analog zum senkrechten Fall. Abbildung 5.15 zeigt die schematische Darstellung des Körpers durch Oberflächenmagnetisierungsströme und die gewählte Nomenklatur.

a.)

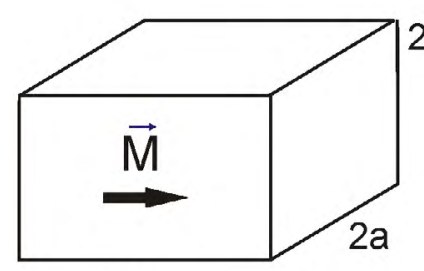

$2 b$ b.)

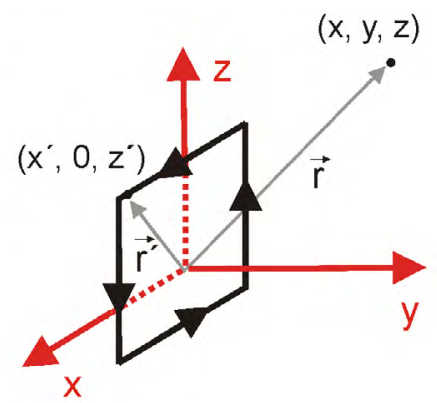

Abbildung 5.15: (a) Schematische Darstellung einer homogenen, rein in der Ebene ausgerichteten Magnetisierung eines Körpers über Oberflächenströme. (b) Skizze zur Festlegung der Nomenklatur.

Als Lösung für die magnetische Induktion in z-Richtung folgt in diesem Fall mit der Remanenzpolarisation $P_{r}$ :

$$
B_{z}(\vec{r})=-\frac{P_{r}}{4 \pi}\left[\left[\left[\ln \left(\sqrt{\alpha^{2}+\beta^{2}+\gamma^{2}}-\alpha\right)-\left(\sqrt{\alpha^{2}+\beta^{2}+\gamma^{2}}+\alpha\right)\right]_{\gamma_{1}}^{\gamma_{2}}\right]_{\alpha_{1}}^{\alpha_{2}}\right]_{\beta_{1}}^{\beta_{2}}
$$

Die Herleitung dieser Formel und die Darstellung der Lösung nach Rücksubstitution findet sich wiederum im Anhang.

Abbildung 5.16 zeigt den Vergleich eines (a) experimentellen Streufeldverlaufes für zwei $10 \mu m \times 10 \mu m$ grosse $\mathrm{PrCo}_{5}-$ Strukturen mit dem eines (b) berechneten Verlaufes. Da die leichten Richtungen der Magnetisierung unter $45^{\circ}$ zur [110] - Richtung stehen (siehe 
a.)

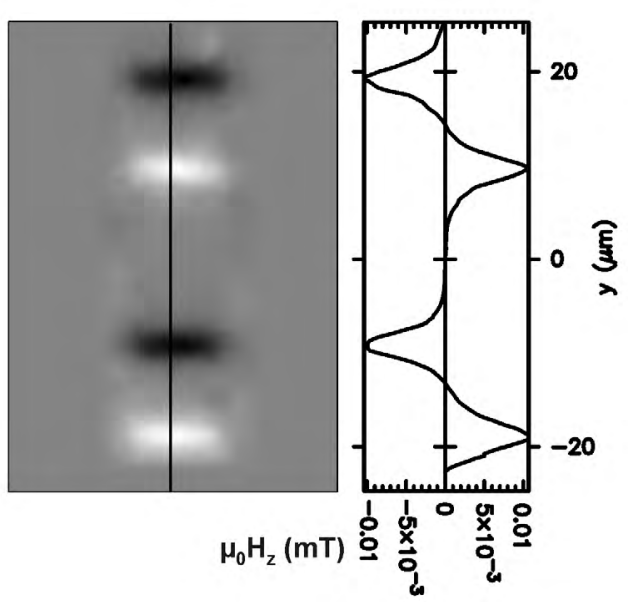

b.)

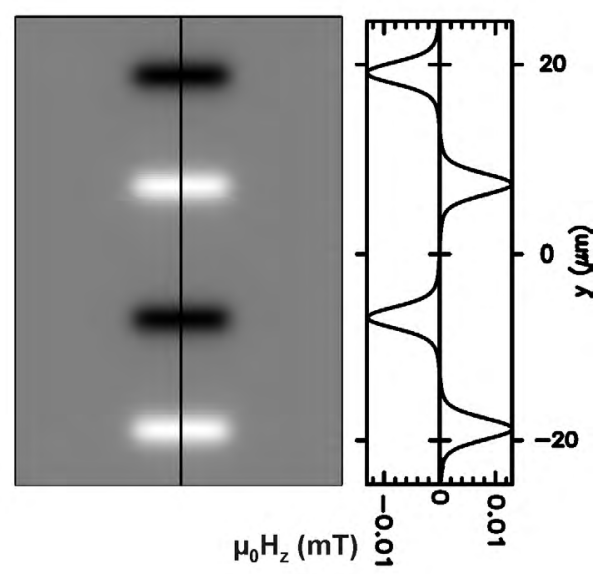

Abbildung 5.16: (a) Experimentelle Streufeldverteilung für zwei $10 \mu \times 10 \mu \mathrm{m}$ große $\mathrm{PrCo}_{5}$ Quadrate. Das Streufeld tritt nur an gegenüberliegenden Kanten auf. Die Probe wurde in einem äußeren Feld von $\mu_{0} H_{\text {ext }}=7 \mathrm{~T}$ in [110]-Richtung aufmagnetisiert. In diese Richtung zeigt ebenfalls die y-Achse des zugrundegelegten Koordinatensystems. (b) Theoretisch berechnete Streufeldverteilung zweier inplane magnetisierter Quadrate mit an die Experimente angepassten Parametern.

Abb. 4.10, Kap. 4.4), bekommt man ein resultierendes Signal aus den $M_{x}$ - $\underline{\text { und }} M_{y}$ Komponenten. Nach der SQUID - Messung an diesem Film (Abb. 4.9) zeigt die Polarisation in dieser Richtung einen Remanenzwert von 0,86 T. Dieser Wert und eine gemessene Filmdicke von $100 \mathrm{~nm}$ wurden für die Simulation des Streufeldverlaufes angesetzt. Die im vorangegangenen Abschnitt erläuterten Korrekturen sind hierbei ebenfalls miteinbezogen worden. Als Parameter dienten wieder Sensordicke $d_{M O L}=600 \mathrm{~nm}$ und Gaußvarianz $\sigma=800 \mathrm{~nm}$. Im Unterschied zu den rein senkrecht aufmagnetisierten Proben tritt in diesem Fall das Streufeld lediglich an den Kanten der Struktur aus. Dies liegt an den senkrecht zur Messebene auftretenden Oberflächenladungen. Da sich die Feldlinien schließen, dreht die $\mathrm{H}_{z}$-Komponente bei reinen inplane Magnetisierungen über bzw. unter der Struktur ihr Vorzeichen um. Der Vergleich zeigt trotz unterschiedlicher Messtopologie gegenüber rein senkrechter Magnetisierung erneut eine ausgezeichnete Übereinstimmung in den Streufeldverläufen, so dass auch hier das im vorherigen Abschnitt entwickelte Bild über den Abbildungsmechanismus und auch die ermittelten Korrekturparameter bestätigt werden. 


\section{Rekonstruktion von Magnetisierungsverteilungen aus Streufeldmessungen}

Die bisher gewonnenen Erkenntnisse zum Abbildungsmechanismus und seine Limitierungen, die in Vorwärtsrechnungen durch entsprechende Korrekturen mathematisch erfasst werden konnten, stellen die Basis für die weitere Magnetisierungsrekonstruktion dar. Ausgangspunkt der folgenden Betrachtungen ist Gleichung 2.20

$$
\begin{aligned}
H_{z}(\vec{k}, z) & =-\frac{\partial}{\partial z} \phi_{M}(\vec{k}, z) \\
& =k \cdot \alpha\left(k, d_{F i l m}, z\right)\left[\sigma_{\perp}(\vec{k})+\frac{\rho_{\|}(\vec{k})}{k}\right],
\end{aligned}
$$

wobei die so definierte Funktion $\alpha\left(k, d_{\text {Film }}, z\right)$ als Feldtransferfunktion bezeichnet wird.

\subsection{Rekonstruktion senkrechter Magnetisierungsverteilungen}

Der Fall für rein senkrechte Magnetisierungsverteilungen lässt sich aus der obigen Gleichung mit $\sigma_{\|}(\vec{k})=0$ auf recht einfache Weise konstruieren. Die Sprungrelation für den Potentialanteil der Magnetisierung lautet im Fourierraum analog zum Realraum

$$
\sigma_{\perp}(\vec{k})=\vec{n} \cdot \vec{M}(\vec{k})=M_{z}(\vec{k})
$$

Der Normalenvektor $\vec{n}$ zeigt dabei auf der oberen (unteren) begrenzenden Fläche in die positive (negative) z-Richtung. Die Ladungsdichteverteilung gibt damit direkt die zKomponente des Magnetisierungsvektors wieder. Dies erlaubt die Möglichkeit, aus der Messung der $H_{z}(\vec{k}, z)$-Komponente die $M_{z}(\vec{k})$-Verteilung mittels

$$
M_{z}(\vec{k})=\frac{H_{z}(\vec{k}, z)}{k \cdot \alpha\left(k, d_{F i l m}, z\right)}=\frac{H_{z}(\vec{k}, z)}{\alpha_{s}\left(k, d_{F i l m}, z\right)}
$$

mit der senkrechten Feldtransferfunktion 


$$
\alpha_{s}(k, d, z)=\frac{e^{-k z}}{2}\left[e^{\frac{k d_{F i l m}}{2}}-e^{-\frac{k d_{F i l m}}{2}}\right]
$$

zu bestimmen. Die Lösungsdarstellung in der von Hug et al. [37] verwendeten Form mittels Transferfunktionen wurde bereits in Kap. 2 angesprochen. Sie sind allgemein durch das Verhältnis der Fourierkomponenten von Streufeld und erzeugender Magnetisierung gegeben und hängen von der räumlichen Frequenz $k$, der Messhöhe $z$ und der Filmdicke $d_{\text {Film }}$ ab. Abbildung 6.1 zeigt die Frequenzabhängigkeit von $\alpha_{s}$ für unterschiedliche Messhöhen bei einer festen Filmdicke von $60 \mathrm{~nm}$. Aus den Transferfunktionen lassen sich einige allgemeine Eigenschaften des Streufeldes ableiten, wenn man eine konstante Magnetisierung im Frequenzraum voraussetzt.

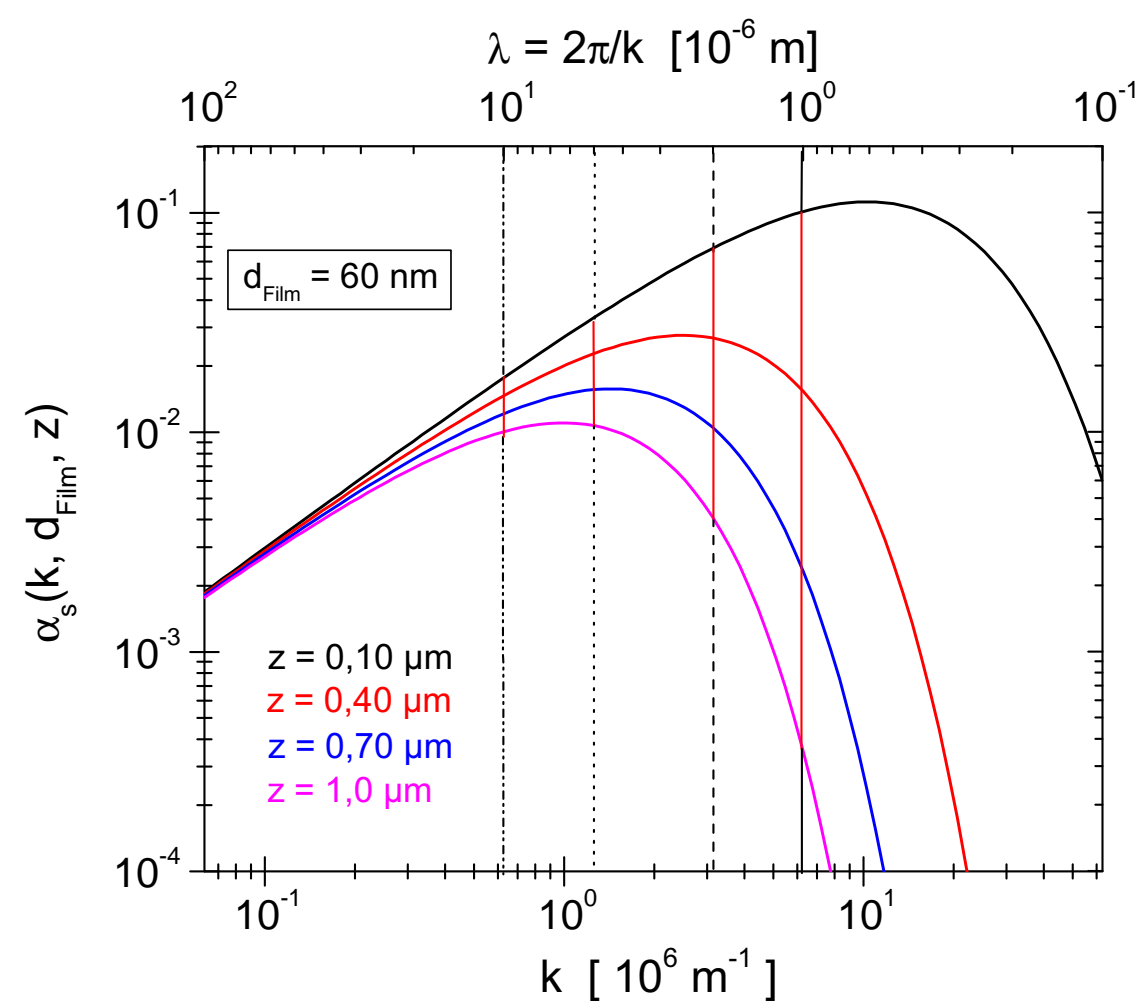

Abbildung 6.1: Senkrechte Feldtransferfunktion eines $60 \mathrm{~nm}$ dicken magnetischen Körpers in Abhängigkeit der räumlichen Frequenz, bzw. Wellenlänge, für verschiedene Messhöhen. Die roten Striche bei ausgewählten Frequenzen markieren den Wertebereich, der durch einen Sensor mit endlicher Dicke von $d_{\text {Sensor }}=900 \mathrm{~nm}$ abgedeckt wird.

Zunächst ist ersichtlich, dass die Feldtransferfunktionen für unterschiedliche Messhöhen vor allem zu großen räumlichen Frequenzen hin einen deutlich voneinander abweichenden Verlauf zeigen. Bei großen Wellenlängen $(>50 \mu \mathrm{m})$ reduziert sich die Messhöhenabhängigkeit und im Grenzwert $k \longrightarrow 0$ strebt $\alpha_{s}$ gegen Null. Die Streufelder über der Mitte einer großen Domäne oder Struktur sind also klein und fallen dafür aber mit der Messhöhe kaum merklich ab. Am Verlauf der $H_{z}(\vec{r})$-Komponente aus Abbildung 5.12 (Kapitel 5.5) kann dies noch einmal gut nachvollzogen werden. Für zunehmende räumliche Frequenzen steigt auch die Transferfunktion an und erreicht ihr Maximum $\left(\partial \alpha_{s} / \partial k=0\right)$ für 
Periodizitätslängen von

$$
\lambda_{\text {max }}=2 \pi d_{\text {Film }} \cdot\left[\ln \frac{z+\frac{d_{\text {Film }}}{2}}{z-\frac{d_{\text {Film }}}{2}}\right]^{-1} .
$$

Daraus folgt, dass kleine Strukturen oder auch schärfere Magnetisierungsänderungen, wie sie an Domänengrenzen auftreten, ein erhöhtes Streufeld generieren und so zu einem erhöhten Kontrast in lokalen streufeldsensitiven Messungen führen. Bei niedrigen Messhöhen $(z=100 \mathrm{~nm})$ ergibt sich ein um bis zu einer Größenordnung stärkeres maximales Feld als bei einer Messhöhe von $1 \mu \mathrm{m}$. Bei kleinen Wellenlängen sieht man, dass die Transferfunktion deutlich abnimmt. Durch Reduktion des Messabstandes kann man die Empfindlichkeit des Verfahrens bei der Streufeldetektion aber etwas erhöhen.

Neben der Höhen- und Frequenzabhängigkeit zeigen sich die Transferfunktion und $\lambda_{\max }$ auch abhängig von der Filmdicke. Die Strukturdicke wurde im Rahmen der Arbeit aber nicht systematisch variiert, so dass diese in den weiteren Charakterisierungen der Transferfunktionen konstant bleibt und an das Beispiel der FePt-Strukturen mit $d_{\text {Film }}=60 \mathrm{~nm}$ angepasst wurde. Es lässt sich jedoch feststellen, dass der Verlauf der Transferfunktion mit zunehmender Dicke $d_{\text {Film }}$ zu größeren Werten verschoben wird und damit auch die Streufelder der entsprechenden Strukturen. $\lambda_{\max }$ reduziert sich dagegen mit wachsendem $d_{\text {Film }}$, so dass das Streufeldmaximum bei kleineren Strukturgrössen erreicht wird.

\section{Rauschunterdrückung und reduziertes Auflösungsvermögen}

Eine inverse Berechnung der Magnetisierung mittels Gl. 6.2 hat eine wesentliche Konsequenz auf die experimentelle Aufösung bei der Rekonstruktion von $\vec{M}(x, y)$-Verteilungen. In Kapitel 5.4 wurde das Aufösungsvermögen bei der Streufeldmessung mit der magnetooptischen Methode bereits ausführlich behandelt und ergab in Abhängigkeit der aktiven Sensorschichtdicke typische Werte von $800 \mathrm{~nm}$ bis etwa 1,3 $\mu \mathrm{m}$. Da bei der Inversion durch die Transferfunktion dividiert wird, kann es aufgrund eines Verstärkens des Rauschanteils in den Messdaten zu einer weiteren Verschlechterung des Auflösungsvermögens kommen. Diese Einschränkung ist in der Literatur bei der Lösung inverser Probleme bereits gut bekannt und wurde in Kap. 2 schon angesprochen. Neben einer experimentellen Reduktion des Rauschanteils, die essentiell für Bilder hoher Qualität und guter Auflösung ist, kann der hochfrequente Anteil elektronisch durch die Multiplikation mit einem Fourierfilter im Frequenzraum unterdrückt werden. Im Ortsraum entspricht dies natürlich wieder einer Faltung mit der Filterfunktion. Ein für die Inversion von MOIF-Daten in vorhergehenden Arbeiten [20, 21, 120, 121] bereits etablierter Filter stellt das sogenannte Hanning-Fenster der Form

$$
w(k)=0,5 \cdot\left[1+\cos \left(\frac{\pi k}{k_{\max }}\right)\right]
$$

dar. Dieses schneidet ab einer maximalen Wellenzahl $k_{\max }$ alle Wellenzahlen $k>k_{\max }$ ab und schwächt auftretende Kanteneffekte durch die Verwendung der Kosinusfunktion ab. Die Einführung der Abschneide-Wellenzahl resultiert in einer Verschlechterung der Ortsauflösung infolge der reduzierten Pixelanzahl in den Bildern. Bei der praktischen Umsetzung der Inversionsrechnungen wird die Grenzfrequenz so lange verändert, bis sich eine 
6. Rekonstruktion von Magnetisierungsverteilungen aus Streufeldmessungen

optimale Güte des Bildes ohne überlagerte Artefakte ergibt. Wie im Folgenden beschrieben, ist die Festlegung von $k_{\max }$ abhängig von der verwendeten Transferfunktion, so dass diese Problematik besonders bei der Berücksichtigung der experimentellen Limitierungen an Bedeutung stark zunimmt.

\section{Endliche Sensordicke und Berechnung der Sensortransferfunktion}

Die eben beschriebenen Feldtransferfunktionen ergeben sich direkt aus den magnetostatischen Grundlagen für das entsprechende Messproblem und können für $H_{z}$-sensitive Abbildungsmethoden als universell angesehen werden. Diese Funktionen berücksichtigen für die Magnetisierungsrekonstruktion die messspezifischen Abbildungsbedingungen jedoch noch nicht. Bei der MOIF-Methode hatte sich in den Vorwärtssimulationen gezeigt, dass die endliche Sensordicke einen erheblichen Einfluss auf die Messdaten hat. Dies ist in den Feldtransferfunktionsverläufen in Abb. 6.1 ebenfalls gut nachvollziehbar. Gerade bei kleineren Wellenlängen stellt das experimentell bestimmte Streufeld eine Mittelung über eine stark messhöhenabhängige Transferfunktion dar. Um dies nochmals zu verdeutlichen, sind in Abb. 6.1 die typischen Strukturgrössen von $10 \mu m, 5 \mu m, 2 \mu m$ und $1 \mu m$ eingezeichnet. Die Messhöhen von $100 \mathrm{~nm}$ bis $1000 \mathrm{~nm}$ repräsentieren hier einen Sensor mit der Dicke $900 \mathrm{~nm}$. Folglich wird bei der MOIF-Messung der $1 \mu \mathrm{m}$ - Quadrate über stärker variierende Streufelder gemittelt als z.B. im Innern einer größeren Struktur. Aber auch hier ist es wichtig zu bedenken, dass die Kanten von großen Strukturen bei der Fouriertransformation auch durch Komponenten mit hohen Wellenzahlen dargestellt werden. Höhere Wellenzahlen haben zwar, wie oben bereits beschrieben, einen höheren Wert der Feldtransferfunktion zur Folge, fallen aber mit zunehmenden Messabstand auch stärker ab. Dies führt zu einem räumlich inhomogenen Abfall des Streufeldes innerhalb der aktiven Sensordicke. Dies ist an dem Beispiel von Strukturen gleicher Kantenlänge mit einer homogenen Magnetisierung $\mu_{0} M_{z}(x, y)=1,4 \mathrm{~T}$ in Abbildung 6.2 noch einmal dargestellt. Die Berechnung des Streufeldes erfolgte in diesem Fall mittels Vorwärtssimulationen gemäß den Formeln in Kapitel 5.5 und ist als Funktion der Messhöhe abgebildet.

Tabelle 6.1 fasst die Werte, um die das Streufeld in der Höhe von $z=100 \mathrm{~nm}$ bis $1 \mu \mathrm{m}$ abfällt noch einmal zusammen. Man sieht, wie deutlich sich die Felddifferenz zwischen dem $1 \mu m$ - Quadrat gegenüber einem $5 \mu m$ - oder dem $10 \mu m$ - Quadrat verändert. Der Inset von Abb. 6.2 zeigt dazu den Streufeldverlauf eines $10 \mu m$ - Quadrates an einer Position $100 \mathrm{~nm}$ in $\mathrm{x}$ - und y-Richtung von einer Ecke entfernt. Die Felddifferenz über die $900 \mathrm{~nm}$ beträgt hier sogar über $150 \mathrm{mT}$.

\begin{tabular}{|c||c|c|c|c|}
\hline Kantenlänge $[\mu m]$ & 1 & 2 & 5 & 10 \\
\hline$\Delta H_{z}[\mathrm{mT}]$ & 57,1 & 20,6 & 2,3 & 0,68 \\
\hline
\end{tabular}

Tabelle 6.1: Gesamtabnahme des Streufeldes über eine Höhendistanz von $z=100 \mathrm{~nm}$ bis $1 \mu \mathrm{m}$ über der Mitte einer Struktur bei verschiedenen Kantenlängen.

Damit bei der Magnetisierungsrekonstruktion aus MOIF-Daten der Effekt der Mittelung mitberücksichtigt wird, ist es sinnvoll eine erweiterte Transferfunktion zu definieren, die darauf basiert, dass wie in Kap. 5 gezeigt, die Magnetisierung der aktiven Sensorschicht 


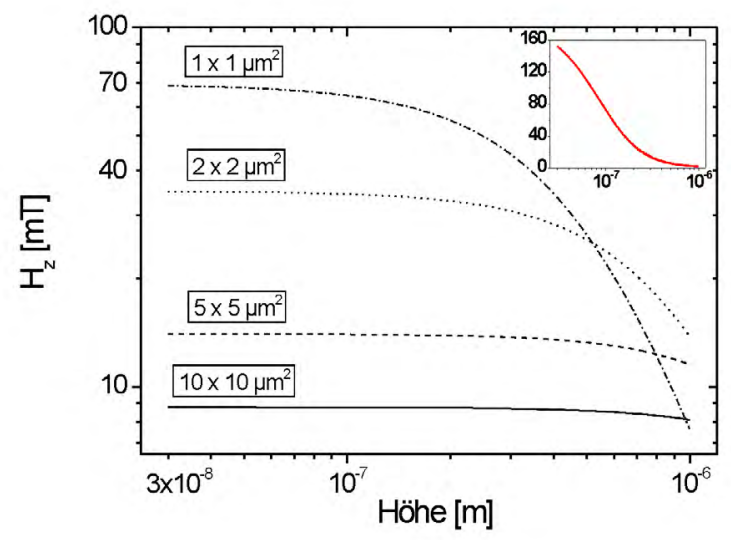

Abbildung 6.2: Theoretische Berechnung der z-Komponente des magnetischen Streufeldes für verschiedene Strukturgrößen als Funktion der Messhöhe. Das Feld wurde direkt über der Mitte der Struktur bestimmt. Der Inset zeigt den höhenabhängigen Verlauf von $H_{z}$ nahe der Ecke einer Struktur (vgl. Schemaskizze neben der Grafik).

in guter Näherung dem lokalen äußeren Feld folgt. Es sei an dieser Stelle noch einmal hervorgehoben, dass gerade in dieser Anpassbarkeit der Transferfunktion an die intrinsichen Messbedingungen die Stärke dieses allgemeinen Formalismus liegt. Aus

$$
\bar{H}_{z}(k, z)=\frac{1}{d_{M O L}} \int_{d_{0}}^{d_{0}+d_{M O L}} H_{z}(k, z) d z
$$

folgt mit Gleichung 6.2

$$
\bar{H}_{z}(k, z)=\frac{1}{d_{M O L}} \int_{d_{0}}^{d_{0}+d_{M O L}} \alpha_{s}\left(k, d_{F i l m}, z\right) \cdot M_{z}(\vec{k}) d z
$$

und nach Ausführung der Integration

$$
\begin{aligned}
\bar{H}_{z}(k) & =\frac{1}{2 d_{M O L}} \frac{e^{\frac{k d_{F i l m}}{2}}-e^{-\frac{k d_{F i l m}}{2}}}{k} e^{-k d_{0}}\left(1-e^{-k d_{M O L}}\right) M_{z}(\vec{k}) \\
& =\beta_{s}(k) \cdot M_{z}(\vec{k}) .
\end{aligned}
$$

$d_{0}-d_{\text {Film }} / 2$ ist die Dicke des Spiegels (30 -40 nm), $d_{0}$ die Höhe, in der die aktive Schicht der Dicke $d_{M O L}$ des Indikators beginnt. Der Verlauf der Transferfunktion $\beta_{s}(k)$, die im Folgenden Sensortransferfunktion genannt wird, ist in Abbildung 6.3 dargestellt.

Zu kleinen Wellenzahlen hin ist der Kurvenverlauf von $\beta_{s}$ analog dem der Feldtransferfunktion, da hier das Streufeld in der relevanten Höhe kaum abfällt. Zu großen Wellenzahlen 


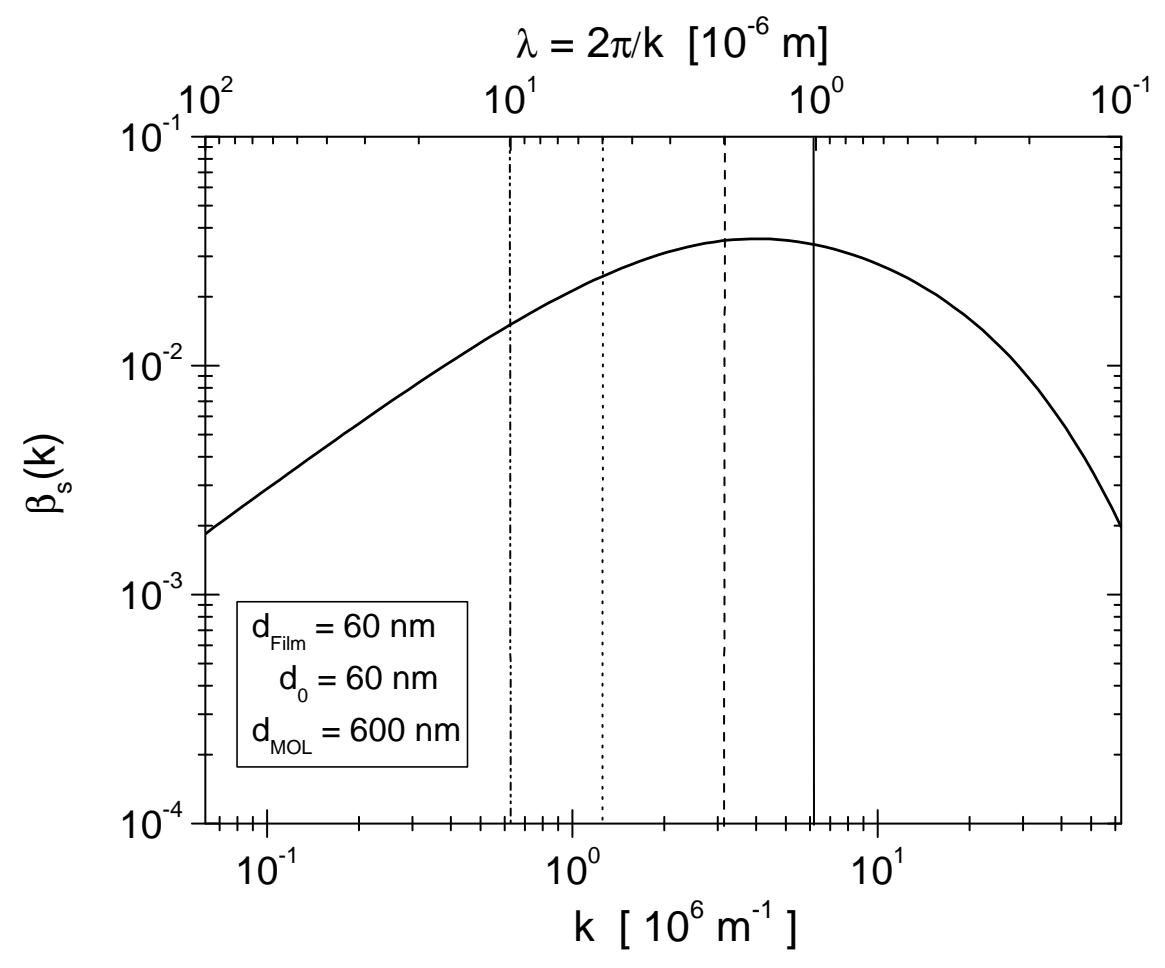

Abbildung 6.3: Senkrechte Sensortransferfunktion eines $60 \mathrm{~nm}$ dicken magnetischen Körpers in Abhängigkeit der räumlichen Frequenz, bzw. Wellenlänge. In die Berechnung gehen gegenüber der Feldtransferfunktion noch die Integrationsparameter $d_{0}=60 \mathrm{~nm}$ als auch die Höhe der ersten Monolage der aktiven Schicht und die Dicke der Sensorschicht $d_{M O L}=600 \mathrm{~nm}$ ein.

hin nehmen die höhengemittelten Streufelder aber nicht mehr so stark ab wie $\alpha_{s}$. Dies führt z.B. dazu, dass die Verstärkung der hochfrequenten Anteile lediglich mäßig ausfällt, da in Gl. 6.7 zur Magnetisierungsberechnung nur durch relativ große Werte dividiert wird. Auf der Sensortransferfunktion beruhende Rekonstruktionen bedürfen daher kaum einer Reduktion der Wellenzahlen und besitzen einen der Originalauflösung äquivalenten Wert. Die vergleichsweise schwache Abhängigkeit von $\beta_{s}$ von der räumlichen Frequenz führt bei der Messung von größeren Strukturen dazu, dass die starken Feldänderungen an den Kanten deutlich abgeschwächt werden. Invertiert man nun das Streufeld von Strukturen unterschiedlicher Kantenlänge

$$
M_{z}(\vec{k})=\frac{\bar{H}_{z}(k, z)}{\beta_{s}(k)},
$$

so erhält man als Ergebnis, am Beispiel von FePt-Quadraten, die in Abbildung 6.4 gezeigte rekonstruierte Magnetisierungsverteilung.

Vergleicht man die experimentell bestimmte Sättigungsmagnetisierung des unstrukturierten Filmes von $\mu_{0} M_{s}=1,4 \mathrm{~T}$ mit den Ergebnissen zu den Remanenzmagnetisierungen aus Rekonstruktion der kalibrierten magneto-optischen Bilder, so zeigt sich bei den größeren Kantenlängen eine hervorragende Übereinstimmung mit den theoretisch erwarteten Werten. Bemerkenswert ist hierbei, dass keine weitere Eichung sowie Anpassung an die experimentellen Daten getätigt wurde. Lediglich die Dimensionen des Sensors, d.h. Spie- 


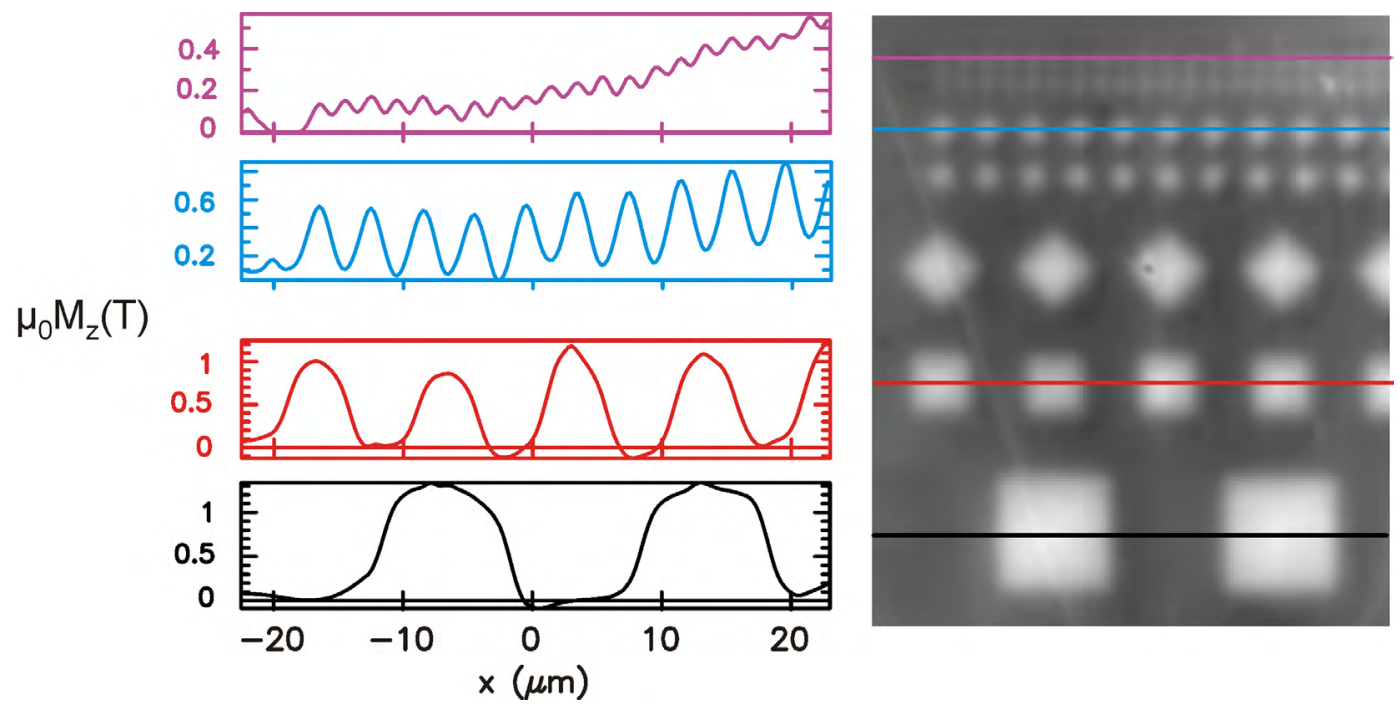

Abbildung 6.4: Darstellung der Remanenzmagnetisierung von FePt-Strukturen verschiedener Kantenlänge (10 $\mu m$ (schwarzes Profil), $5 \mu m$ (rot), $2 \mu m$ (blau) und $1 \mu m$ (magentafarben)) nach Inversion mit der Sensortransferfunktion. SQUID-Messungen an dem Film hatten eine Sättigungsmagnetisierung von etwa 1,4 T gezeigt (Abb. 4.6).

geldicke und aktive Sensordicke, sowie die Filmdicke müssen bekannt sein. Die kleineren Strukturen weisen dagegen eine zu kürzeren Kantenlängen deutlich zunehmende Abweichung von den Referenzwerten auf. Die $2 \mu m$-Quadrate besitzen nach Maßgabe der Inversionsrechnung nur noch eine Remanenz von etwa 0,5 - 0,6 T und die $1 \mu m$-Quadrate gar eine von 0,1 - 0,2 T. Hier ist eindeutig erkennbar, dass die Rekonstruktion mittels Sensortransferfunktion nicht alle realen Messbedingungen beinhaltet und besonders für kleine Wellenlängen die Berücksichtigung der begrenzten lateralen Ortsauflösung erforderlich ist.

\section{Experimentelle Auflösung und Berechnung der Abbildungstransferfunktion}

Wie die simulierten Streufeldberechnungen bereits gezeigt hatten, nimmt der Einfluss des begrenzten magnetischen Aufösungsvermögens gerade für die kleinen Strukturen deutlich zu und resultieren neben der endlichen Sensordicke in einer weiteren Reduktion des Messsignals. Die Verwendung einer Gaußverteilung zur Simulation dieses Effektes mit einer, diesem Auflösungsvermögen angepassten, Varianz hatte sich dabei als physikalisch sinnvoll erwiesen. Um zunächst die Auswirkungen auf die Sensortransferfunktion und damit auf die Magnetisierungsbestimmung zu simulieren, kann man nun die im Ortsraum normierte Gaußfunktion (vgl. Gl. 5.16) auch in den Fourierraum transformieren, wobei die Faltung vom Messsignal mit der Auflösungsfunktion in eine Multiplikation übergeht. Die Gaußfunktion besitzt im Frequenzraum eine dem Realraum ähnliche Darstellung,

$$
G_{\sigma}\left(k_{x}, k_{y}\right)=e^{-\frac{k^{2} \sigma^{2}}{2}}
$$

mit $k^{2}=\left(k_{x}^{2}+k_{y}^{2}\right)$ und der Varianz $\sigma^{-1}$. Die resultierende Abbildungstransferfunktion ergibt sich somit zu 


$$
\gamma_{s}(k, \sigma)=\beta_{s}(k) e^{-\frac{k^{2} \sigma^{2}}{2}}
$$

Abbildung 6.5 zeigt $\gamma_{s}(k, \sigma)$ für die bereits oben verwendeten Parameter und bei fester Varianz für die Gaußfunktion von $\sigma=800 \mathrm{~nm}$.

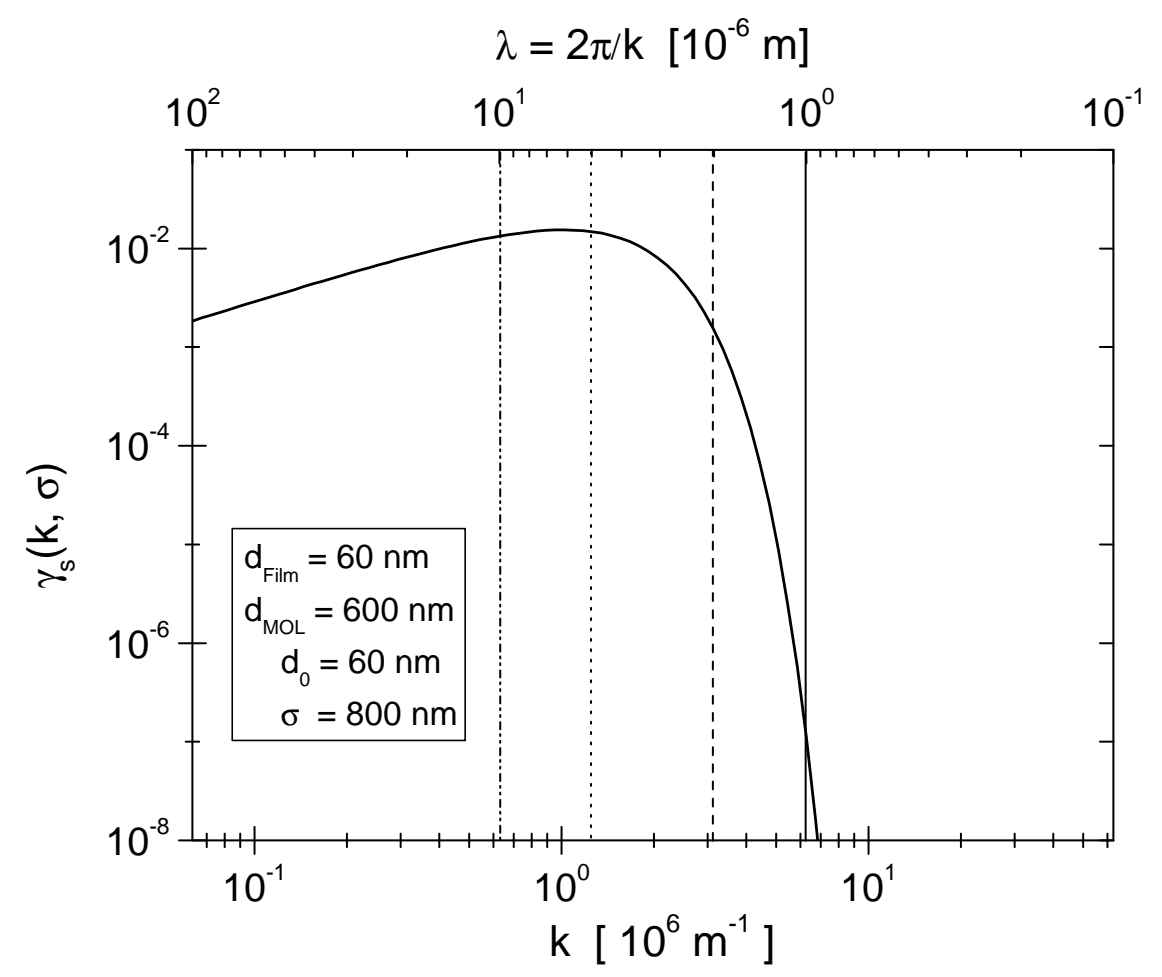

Abbildung 6.5: Senkrechte Abbildungstransferfunktion eines $60 \mathrm{~nm}$ dicken magnetischen Körpers in Abhängigkeit der räumlichen Frequenz, bzw. Wellenlänge. Als Erweiterung zur Sensortransferfunktion berücksichtigt $\gamma_{s}$ die experimentell begrenzte Auflösung mittels einer Gaußschen Unschärfe. $\sigma=800 \mathrm{~nm}$ stellt die Varianz der in die Rechnungen mit eingehenden Gaußkurve dar.

Die Berücksichtigung der endlichen Auflösung führt wieder zu einer $k$-Abhängigkeit, die noch stärker ausgeprägter ist als bei $\alpha_{s}$ und bei $\beta_{s}$ durch die Sensormittelung deutlich relativiert wurde. Dadurch ist $\gamma_{s}$ bei großen k-Werten erheblich reduziert, bei kleinen ergeben sich nur leichte Korrekturen. Dies ist auch der Grund, warum die $10 \mu \mathrm{m}$ - Quadrate schon bei der Sensortransferfunktion eine so gute Übereinstimmung nach der Berechnung der Remanenzmagnetisierung zeigten. Bei einer Wellenlänge von $5 \mu \mathrm{m}$ erkennt man an $\gamma_{s}$ schon den zunehmenden Einfluss der beschränkten Auflösung. Während die Sensortransferfunktion bei dieser Wellenlänge im Wert noch ansteigt, fällt er für $\gamma_{s}$ schon ab. Im Bereich $5 \geq \lambda \geq 2 \mu m$ sinkt die Abbildungstransferfunktion dann bereits um eine Größenordnung in ihrem Wert, bevor sie für noch kleinere Wellenlängen um mehrere Zehnerpotenzen einbricht. Dies bestätigen auch die experimentellen Ergebnisse aus dem Vorwärtssimulationskapitel, in dem der Einbruch des magnetischen Streufeldes vor allem bei den gemessenen $1 \mu \mathrm{m}$ Strukturen zu erkennen war. Für die Rauschunterdrückung stellt der Verlauf von $\gamma_{s}$ ein erhebliches Problem dar. Die Division durch die verschiedenen Transferfunktionen bei der Rekonstruktion der Magnetisierung führt aufgrund von 
$\gamma_{s}(k)<<\beta_{s}(k)$ und $\gamma_{s}(k)<<\alpha_{s}(k)$ bei gleichen Wellenzahlen dazu, dass die Rauschverstärkung bei der Abbildungstransferfunktion wesentlich deutlicher auftritt. Aufgrund der kleinen Werte für moderate Frequenzen müssen recht hohe Abschneide-Wellenzahlen verwendet werden, d.h. ein erheblicher Anteil der in den Bildern enthaltenen hohen Frequenzen wird unterdrückt. Dies wirkt sich im Folgenden deutlich einschränkend auf die Rekonstruierbarkeit der Magnetisierung kleiner Strukturen aus.

Wendet man nun die Abbildungstransferfunktion zur Rekonstruktion der Magnetisierung für die oben gezeigten FePt-Strukturen an, ergibt sich die in Abbildung 6.6 dargestellte $M_{z}(x, y)$-Verteilung.

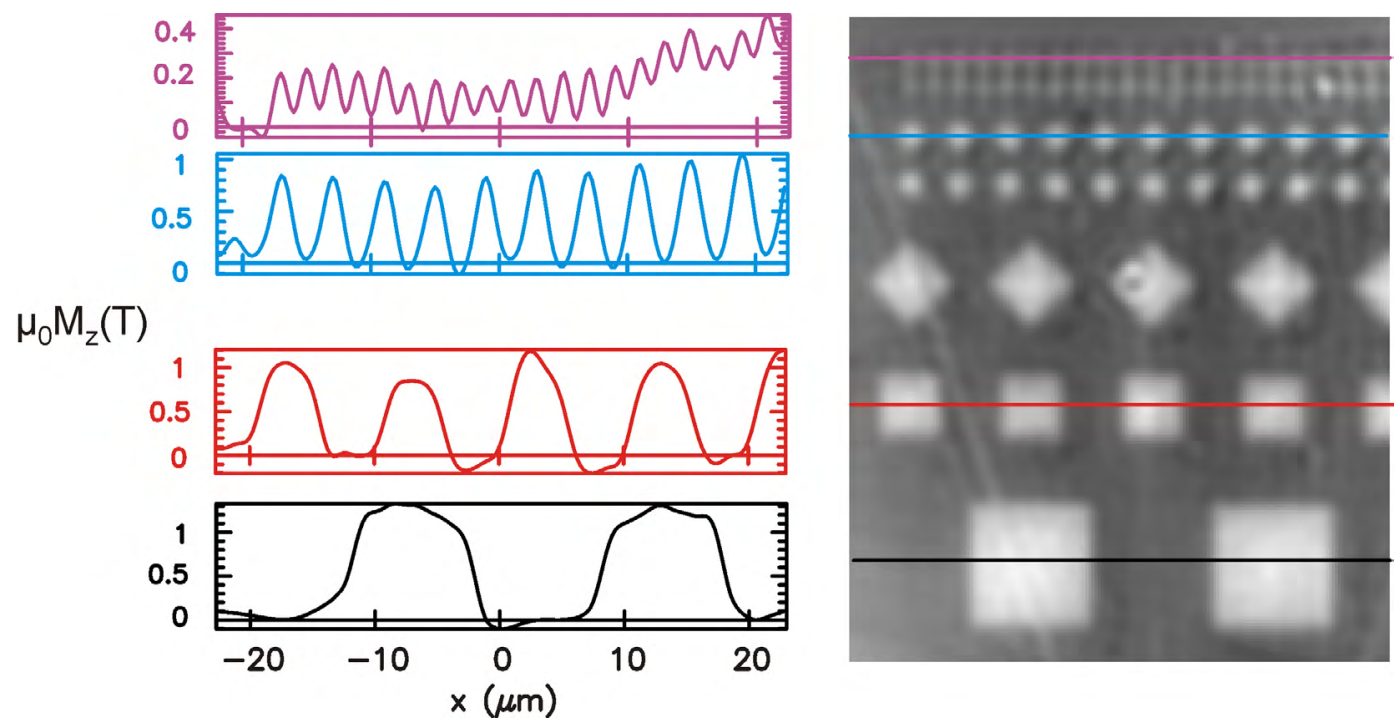

Abbildung 6.6: Darstellung der Remanenzmagnetisierung von FePt-Strukturen gleicher Kantenlänge wie in Abb. 6.4 nach Inversion mit der Abbildungstransferfunktion.

Es ist erkennbar, dass die Remanenzmagnetisierungen der Quadrate mit $10 \mu \mathrm{m}$ und $5 \mu \mathrm{m}$ Kantenlänge unverändert geblieben sind. Im Vergleich zu Abb. 6.4 weisen jedoch die $2 \mu m$ Strukturen mit 0,9 - 1,0 T bereits erhöhte Werte auf, erreichen den Referenzwert aber auch nicht vollständig. Die kleinsten Strukturen zeigen mit Maximalwerten zwischen 0,2 - 0,3 T nur eine schwache Veränderung und liegen weit von den Referenzen entfernt. In Abbildung 6.6 resultiert die Rauschunterdrückung in einer reduzierten Ortsauflösung von 1,67 $\mu m$, wohingegen diese in den Ausgangsbildern etwa $800 \mathrm{~nm}$ beträgt. Die nach dem Festlegen der Abschneide-Wellenzahl resultierende effektive Ortsauflösung wird im Folgenden als $\lambda_{\min }=2 \pi / k_{\max }$ bezeichnet. An dieser Stelle zeigt sich bereits, dass eine korrekte Rekonstruktion von Strukturen mit $1 \mu m$ Kantenlänge unter den obigen Messbedingungen schwer realisierbar ist.

\section{Einfluss der Rauschunterdrückung auf die Magnetisierungsrekonstruktion}

Um die Grenzen der Rekonstruierbarkeit, die durch die inverse Problematik bestimmt wird, quantitativ besser bestimmen zu können, sollen die Auswirkungen des Filtereffekts 
detaillierter analysiert werden. Dazu wurden vorwärtssimulierte Streufeldberechnungen an Quadraten verschiedener Kantenlängen durchgeführt und diese wieder invertiert, um die Magnetisierung nach der Inversion zu bestimmen. Hierbei wurde analog zu den Rechnungen aus Kapitel 5.5 vorgegangen und die entsprechenden Korrekturen mitberücksichtigt. Die Werte, die in die Vorwärtsrechnungen eingehen, werden auch in die Abbildungstransferfunktion eingesetzt. Es zeigt sich, dass die rekonstruierte Magnetisierung bei fester Kantenlänge der Quadrate abhängig von der Abschneide-Wellenzahl, also der effektiven Ortsauflösung, ist. Abbildung 6.7 gibt die Differenz $\Delta \mu_{0} M_{z}$ zwischen der Referenzmagnetisierung $\mu_{0} M_{0, z}=1,4 \mathrm{~T}$ und der nach Inversion rekonstruierten Magnetisierung $\mu_{0} M_{i n v, z}$ in Abhängigkeit der effektiven Ortsauflösung an.

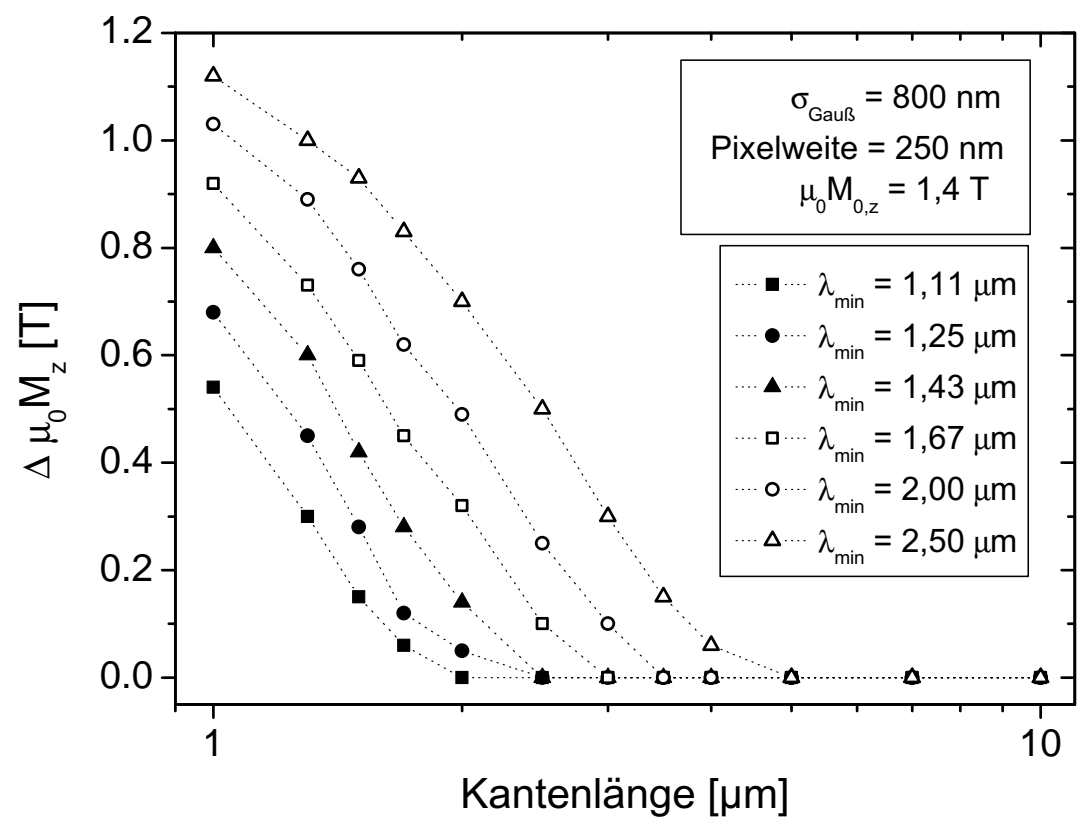

Abbildung 6.7: Differenz $\Delta \mu_{0} M_{z}$ zwischen rekonstruierter Magnetisierung und Referenzwert, die sich nach theoretischen Simulationen ergibt, als Funktion der Kantenlänge simulierten Quadrate. Die Berechnung der Verläufe ist für verschiedene effektive Ortsauflösungen $\lambda_{\text {min }}$, die sich nach der Fourierfilterung ergeben, durchgeführt. Die von der CCD-Kamera und dem verwendeten Objektiv gegebene theoretische Pixelweite und die Varianz der Gaußschen Unschärfe sind dabei an die experimentellen Daten angepasst. Die gestrichelten Linien sind lediglich zur besseren Visualisierung verwendet und stellen keine analytische Funktion dar.

Man erkennt, dass mit zunehmender Reduktion der Ortsauflösung die Abweichung von $\mu_{0} M_{i n v, z}$ gegenüber der Referenzmagnetisierung zunimmt. Der Wert der Kantenlänge, bei dem die Kurve von Null abweicht, wird im Weiteren als kritische Kantenlänge bezeichnet. Diese Grenzlänge verschiebt sich mit steigendem Wert für $\lambda_{\min }$ zu sukzessive größeren Kantenlängen. Bei den dargestellten Verläufen erweist sich dabei $5 \mu m$ als kritischer Wert bis zu der die Magnetisierung aller Strukturen vollständig rekonstruiert werden kann. Zu besseren Ortsauflösungen hin verschiebt sich diese Grenze bis zu einer Schwelle von 2 $\mu m$, die unter den vorgegenenen externen Parametern die Obergrenze für eine korrekte Rekonstruktion darstellt. Die Verwendung anderer Referenzmagnetisierungen wie z.B. $\mu_{0} M_{0, z}=1 \mathrm{~T}$ und 0,75 T führt zu einem nahezu gleichen Ergebnis, was in Abbildung 6.8 
deutlich wird. Da der Absolutwert der Differenz zwischen Ausgangs- und rekonstruierter Magnetisierung abhängig vom Referenzwert selber ist, wurde hier das Verhältnis beider Größen in einer normierten Darstellung aufgetragen. In Abb. 6.8(a) ist zu erkennen, dass die Verläufe bei fester effektiver Ortsauflösung nahezu unabhängig von der zugrundegelegten Ausgangsmagnetisierung sind. Es liegt damit in dem verwendeten Parameterbereich ein fast universelles Verhalten vor. Die kritische Kantenlänge zeigt sich einzig von der auftretenden Ortsauflösung beeinflusst. Kennt man diese, läßt sich daraus ein Vertrauensbereich für die Inversion der Streufelder abschätzen.

Abbildung 6.8(b) zeigt den Vergleich der in (a) zu fester Auflösung gemittelten Kurvenverläufe, mit den aus Abbildung 6.6 experimentell ermittelten Werten für die FePtStrukturen. Letztere zeigen sich gegenüber den theoretischen leicht reduziert, aber immer noch in guter Übereinstimmung. Die Fehlergrenzen ergeben sich aus den Unsicherheiten bei der Streufeldbestimmung und der daraus resulierenden Magnetisierung, die bei ca. $\Delta \mu_{0} M=0,1 \mathrm{~T}$ liegen und in Relation zum Referenzwert in der normierten Darstellung etwa $\pm 0,07$ ausmachen.

Zusammenfassend zeigt sich also, dass die Rekonstruierbarkeit der Magnetisierung von Strukturen abhängig von der effektiven Ortsaufösung nach der Inversion ist. Müssen aufgrund eines zu hohen Rauschanteils sehr viele hochfrequente Anteile unterdrückt werden, fehlen in dem invertierten Magnetisierungsbild die zur Fourierdarstellung kleiner Strukturen notwendigen hohen räumlichen Frequenzen. Daraus resultieren die viel zu kleinen Werte für $M_{i n v}$ und es ergibt sich eine Grenze für Kantenlängen, bis zu der eine korrekte Rekonstruktion möglich ist. Darüber hinaus kann Abb. 6.8(b) dazu genutzt werden, für Strukturen mit Kantenlängen jenseits des kritischen Wertes eine Abschätzung für die zugrundeliegende Magnetisierung zu geben, indem die Werte zu fester Auflösung als Korrektur- bzw. Skalierungsfaktor verwendet werden. Dies führt allerdings bei kleineren Strukturgrößen zu steigenden relativen Fehlern.

\section{Abhängigkeit der Rekonstruktion von optimalen Messbedingungen}

In den bisherigen Abschnitten hatte sich gezeigt, dass die Messbedingungen einen wesentlichen Einfluss auf die Rekonstruktion der Magnetisierung haben. Vor allem das begrenzte Auflösungsvermögen bestimmt aufgrund der daraus resultierenden starken Frequenzabhängigkeit der Abbildungstransferfunktion die Grenze der Rekonstruierbarkeit. In diesem Abschnitt soll auf Basis des entwickelten Transferfunktionenformalismus für die MOIFMethode, ähnlich dem experimentellen Kapitel 5.4, eine Optimierung der einzelnen Abbildungsparameter diskutiert und die theoretisch erreichbare Grenze der Magnetisierungsrekonstruktion ermittelt werden.

Die effektive Ortsaufösung nach dem Fourierfiltern hatte sich am Beispiel der FePtStrukturen in Abb. $6.6 \mathrm{zu} \lambda_{\text {min }}=1,7 \mu \mathrm{m}$ ergeben. Dieser Wellenlänge entspricht unter Berücksichtigung der Referenzmagnetisierung von $\mu_{0} M_{z}(k)=1,4 \mathrm{~T}$ und der Verwendung der Abbildungstransferfunktion in Abb. 6.5 ein minimaler Streufeldwert von $\mu_{0} H_{z}\left(\lambda_{\text {min }}\right)=3 \cdot 10^{-1} \mathrm{mT}$ oder ein Wert der Transferfunktion von $\gamma_{s}\left(\lambda_{\text {min }}\right)=4,2 \cdot 10^{-4}$, der gerade noch darstellbar ist. Dieser Grenzwert ist in Abbildung 6.9 durch die gestrichelte Linie gekennzeichnet. Die drei Abbildungen zeigen die senkrechte Abbildungstransferfunktion für verschiedene Sensorschichtdicken, Spiegeldicken und Ortsauflösungen. 

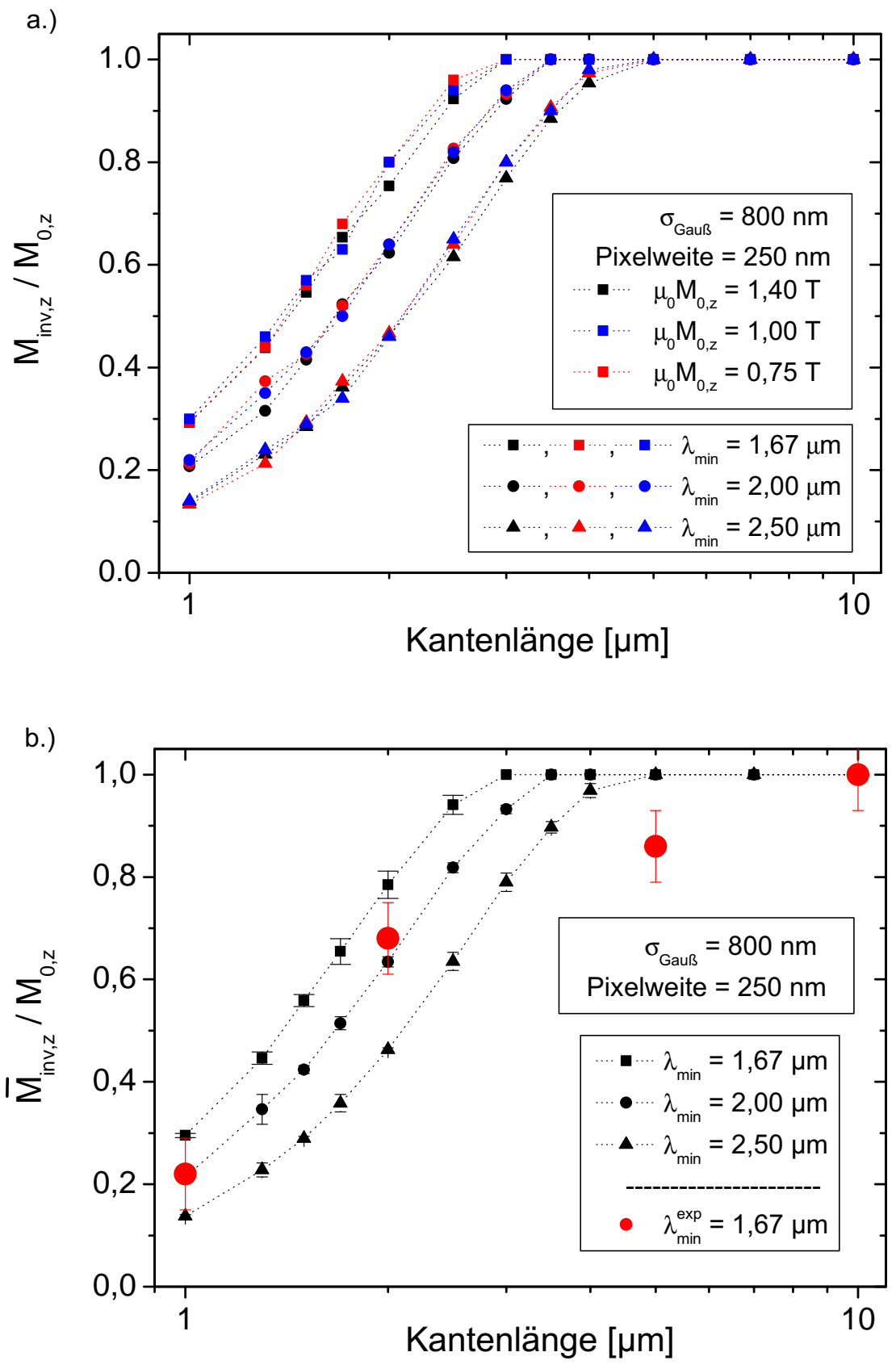

Abbildung 6.8: (a) Verhältnis aus rekonstruierter zu Referenzmagnetisierung als Funktion der Kantenlänge der simulierten Quadrate. Dieses ist berechnet für verschiedene Ortsauflösungen und Referenzmagnetisierungen. (b) Mittelwert der invertierten Magnetisierung aus den drei Kurven zu jeder Auflösung, aufgetragen gegen die Kantenlänge der Quadrate. Als rote Punkte mit dargestellt sind die experimentell bestimmten Daten aus Abb. 6.6.

Man erkennt, dass die Variation der aktiven Schichtdicke nur einen geringen Einfluss auf den Verlauf von $\gamma_{s}(k, \sigma)$ hat, wobei die Tendenz zu einem schwachen Anstieg der Abbildungstransferfunktion mit sinkender Sensordicke geht. Je dünner die aktive Indikatorschichtdicke ist, desto mehr wird nur über größere Feldwerte gemittelt. Die Schnittpunkte der Kurven mit der Grenzlinie sind in Tabelle 6.2 dargelegt und zeigen eine nur eine 

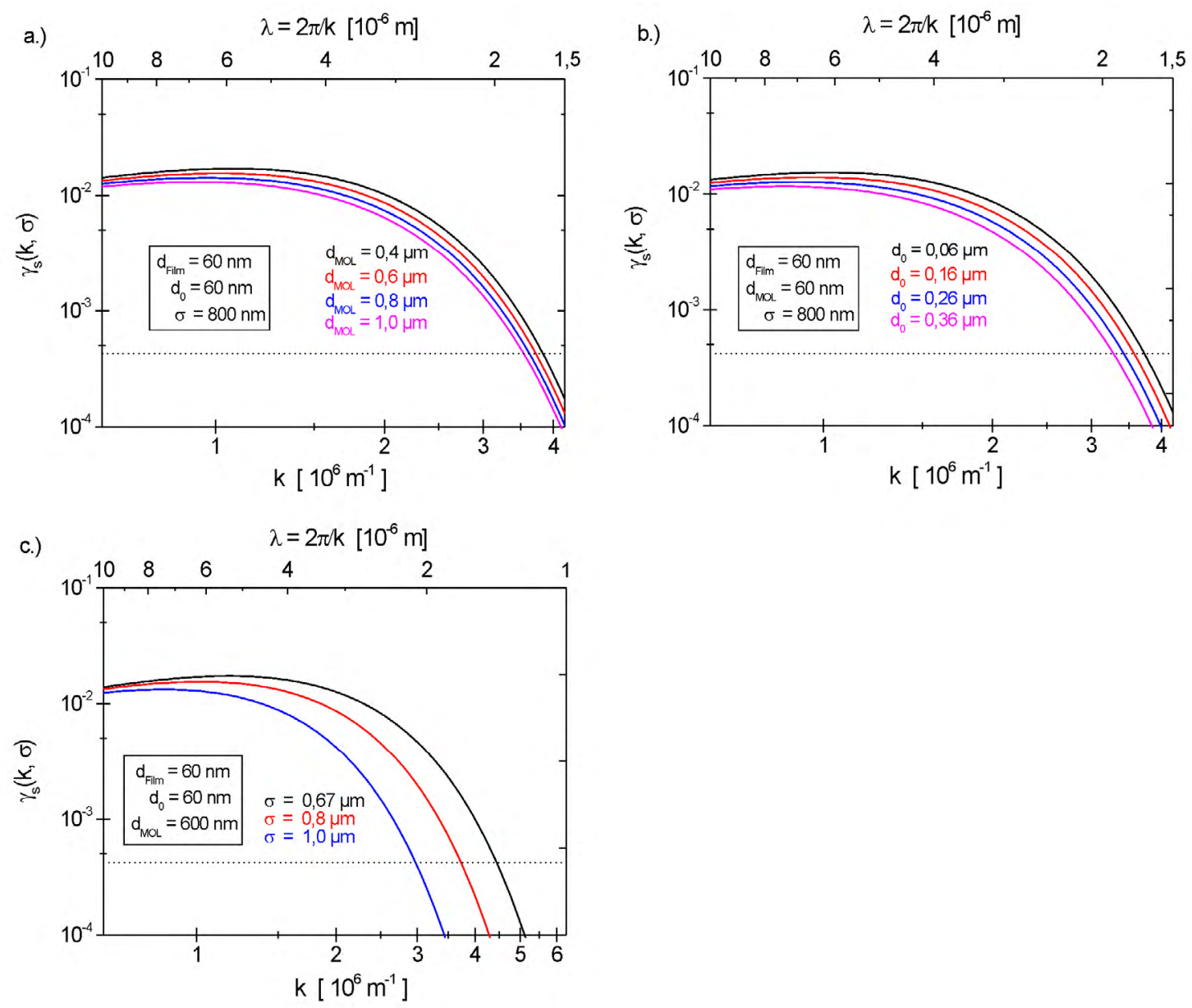

Abbildung 6.9: (a) Abbildungstransferfunktion aufgetragen gegen die räumliche Frequenz, bzw. Wellenlänge, für verschiedene aktive Sensorschichtdicken. (b) Analoge Auftragung für verschiedene Spiegeldicken, bzw. minimale Abstände zwischen aktiver Schicht und Strukturoberfläche, sowie (c) für verschiedene theoretische Ortsauflösungen. Die zusätzlich zur Simulation verwendeten Parameter sind in den Kästchen angegeben.

schwache Abhängigkeit der Aufösungsgrenze von der aktiven Dicke des Sensors.

\begin{tabular}{|c||c|c|c|c|}
\hline$d_{M O L}[\mu m]$ & 0,4 & 0,6 & 0,8 & 1,0 \\
\hline$\lambda_{\min }[\mu m]$ & 1,64 & 1,70 & 1,74 & 1,79 \\
\hline
\end{tabular}

Tabelle 6.2: Auswertung der Abbildungstransferfunktion für unterschiedliche theoretische Auflösungen, die sich aus dem Abschneiden von Wellenzahlen zur Fourierfilterung bei variierender Sensordicke ergeben. Als minimaler noch zulässiger Wert für die Abbildungstransferfunktion bei entsprechender Frequenz wurde $\gamma_{s}=4,2 \times 10^{-4}$ abgeschätzt.

In Abbildung 6.9(b) wurde der Abstand der untersten Monolage der aktiven Sensorschicht variiert. Hierbei zeigt sich analog, dass die Abbildungstransferfunktion höhere Werte annimmt, wenn die aktive Schicht nahe der zu untersuchenden Strukturoberfläche ist. Die Spiegeldicke sollte also so gering wie möglich gewählt werden aber noch ein gutes Reflexionsvermögen besitzen. 


\begin{tabular}{|c||c|c|c|c|}
\hline$d_{0}[\mu m]$ & 0,06 & 0,16 & 0,26 & 0,36 \\
\hline$\lambda_{\min }[\mu m]$ & 1,69 & 1,76 & 1,84 & 1,92 \\
\hline
\end{tabular}

Tabelle 6.3: Auswertung der Abbildungstransferfunktion bei variierender Spiegeldicke analog zu Tabelle 6.2.

Auch wenn die Abhängigkeit der Abbildungstransferfunktion von den beiden Parametern eher als schwach zu bezeichnen ist, muss unbedingt der Einfluss dieser beiden Größen auf das experimentelle Auflösungsvermögen mitberücksichtigt werden (vgl. Kap. 5.4), da dies wiederum die deutlichsten Veränderungen auf den Verlauf von $\gamma_{s}$ hat (siehe Abb. 6.9). In der Varianz der Gaußkurve sind die oben dargestellten Größen $d_{M O L}$ und $d_{0}$ also schon implizit enthalten. Die Abbildungstransferfunktion ist für drei verschiedene Aufösungen bei Verwendung der optimierten Parameter aus (a) und (b) dargestellt. Der Wert von $\sigma$ $=670 \mathrm{~nm}$ stellt dabei die Grenze des theoretischen Auflösungsvermögens dar.

\begin{tabular}{|c||c|c|c|}
\hline$\sigma[\mu m]$ & 0,67 & 0,8 & 1,0 \\
\hline$\lambda_{\min }[\mu m]$ & 1,42 & 1,69 & 2,12 \\
\hline
\end{tabular}

Tabelle 6.4: Auswertung der Abbildungstransferfunktion bei variierender Ortsauflösung.

Unter den gegebenen Bedingungen stellt eine Ortsaufösung von $\lambda_{\min }=1,42 \mu \mathrm{m}$ die Grenze dar, die nach der Fourierfilterung maximal erreichbar scheint. Die Rekonstruktion der Magnetisierung aus theoretisch berechneten Streufeldverteilungen unter Verwendung dieser optimalen Parameter ergibt für verschiedene Strukturgrößen den in Abbildung 6.10 gezeigten Verlauf (analog zu Abb. 6.8). Aufgetragen ist wieder das Verhältnis aus invertierter zu Referenzmagnetisierung als Funktion der Kantenlänge der Strukturen. Als minimale, noch korrekt erfassbare Strukturgröße erhält man demnach $2 \mu \mathrm{m}$.

Zuletzt soll noch eine weitere Limitierung diskutiert werden, die durch die Sensitivität des Indikators auftritt und gerade bei kleinen Strukturen einen Fehler bei der Streufeldbestimmung und damit bei der Inversion hervorrufen kann. Als Beispiel sei der $600 \mathrm{~nm}$ dünne Indikator gegeben, der bei einem externen Streufeld von $H_{z}=60 \mathrm{mT}$ sättigt. Bei diesem Feld ist die Magnetisierung vollständig in z-Richtung ausgerichtet, so dass höhere Felder kein weiteres Signal mehr generieren.

Abbildung 6.11 zeigt das senkrechte Streufeld in Abhängigkeit der räumlichen Frequenz, das sich aus Gleichung 6.2 nach Multiplikation der Feldtransferfunktion $\alpha_{s}$ mit einer konstanten Magnetisierung von $M_{z}(k)=1,4 \mathrm{~T}$ in zwei verschiedenen Messhöhen ergibt. Hierbei ist zu berücksichtigen, dass mit dieser Multiplikation im Wesentlichen nur die Hauptfourierkomponente einer rechteckigen Struktur variiert wird, höhere Fourierkomponenten besitzen kleinere Koeffizienten. Da die Hauptkomponente jedoch den entscheidenden Beitrag zur Rekonstruktion liefert, haben die weiteren Betrachtungen einen wesentlichen Einfluss auf die Quantifizierung der Magnetisierung. Betont sei noch einmal, dass im weiteren nur die reinen Feldwerte der Strukturen ohne experimentelle Limitierungen diskutiert werden. Die Daten wurden dabei an das Beispiel FePt angepasst. Eine minimale Messhöhe von $z=60 \mathrm{~nm}$ führt für kleine Wellenlängen, $\lambda<3,9 \mu \mathrm{m}$, zu Streufeldern, die das Sättigungsfeld des Indikators überschreiten (schwarz schraffierter Bereich). Erst bei 


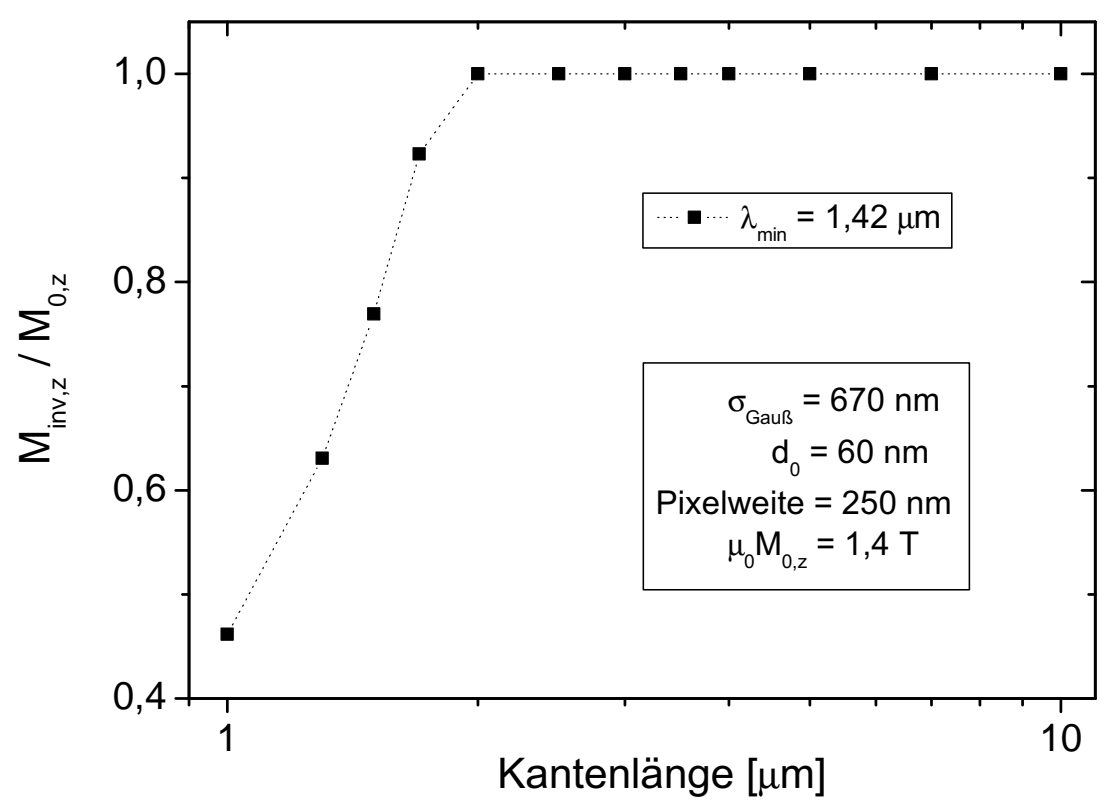

Abbildung 6.10: Verhältnis aus rekonstruierter zu Referenzmagnetisierung als Funktion der Kantenlänge der simulierten Quadrate analog zu Abb. 6.8. In diesem Fall wurde die Inversion unter optimalen Messbedingungen simuliert. Damit stellt der Wellenlängenbereich zwischen 1,5 - $2 \mu \mathrm{m}$ die experimentelle Grenze der vollständigen Rekonstruierbarkeit von Magnetisierungsverteilungen mittels MOIF dar.

einer Messhöhe von $z=260 \mathrm{~nm}$ wird dieser Wert für alle Wellenzahlen unterschritten. Dies bedeutet, dass die Streufelder bei Wellenzahlen, die maßgeblich zur Rekonstruktion von Strukturen mit Kantenlängen $<4 \mu \mathrm{m}$ beitragen, in den ersten ca. $200 \mathrm{~nm}$ des Indikators nicht exakt in eine korrekte Faraday-Drehung umgesetzt werden. Die Abweichung wird jedoch durch die begrenzte experimentelle Auflösung nach der Inversion reduziert (rot schraffierter Bereich), da durch Festlegen der Abschneide-Wellenzahl nur Wellenlängen im Bereich von $\lambda_{\min } \approx 3,9-1,7 \mu \mathrm{m}$ beitragen. Die Feldwerte, die sich nun aus den über die aktive Sensordicke gemittelten Streufeldern ergeben, weisen damit eine von der Strukturgröße abhängige Unterschätzung auf. Während größere Strukturen mit Kantenlängen von 5 - $10 \mu m$ lediglich an den Rändern geringfügige Abweichungen aufweisen, die ohnehin durch die begrenzte räumliche Auflösung aufgeschmiert sind, kann der Effekt bei den $2 \mu m$-Strukturen bereits zu einer Unterschätzung der Streufeldwerte von bis zu $20 \%$ führen. Vergleicht man diesen relativen Fehler mit jenem, der in Abb. 6.8(b) zwischen dem theoretischen und dem experimentellen Magnetisierungswert des $2 \mu \mathrm{m}$-Quadrates auftritt, so findet sich hier ebenfalls eine Abweichung von $\approx 20 \%$. Diese begründet sich damit sehr wahrscheinlich aus der Sättigung des Indikators in den ersten $200 \mathrm{~nm}$ der aktiven Schichtdicke. Bei dem $1 \mu m$ Quadrat ist der Fehler noch größer, da aber die relevanten Wellenlängen durch die Filterung abgeschnitten werden, lässt sich dieser nicht explizit abschätzen, sondern hängt von der Filterung selbst ab. Bei der prinzipiellen Bewertung dieser Fehlerquelle ist jedoch zu bedenken, dass mit der sehr hohen Sättigungsmagnetisierung im FePt in dieser Arbeit ein Extrembeispiel gewählt wurde. Bereits beim CoPt mit einem $M_{s}=1 \mathrm{~T}$ beträgt die Unterschätzung der Streufeldwerte für Strukturen mit Kantenlängen von $2 \mu \mathrm{m}$ lediglich noch 5\%. Durch Verwendung von Sensoren mit höherem Sättigungs- 


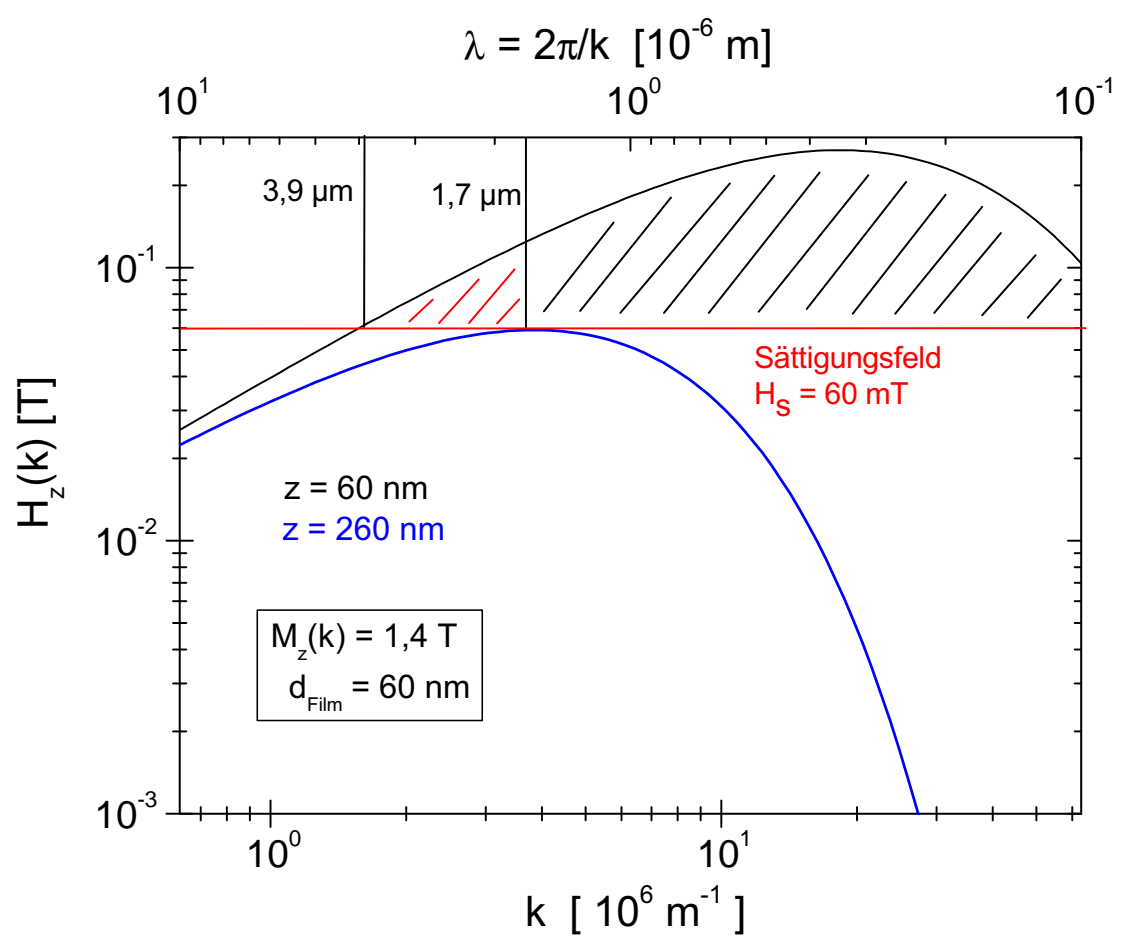

Abbildung 6.11: Verlauf des Streufeldes in Abhängigkeit der räumlichen Frequenz am Beispiel von $60 \mathrm{~nm}$ dickem FePt mit einer konstanten Sättigungsmagnetisierung von 1,4 T. Die rote Linie markiert das Sättigungsfeld des verwendeten $600 \mathrm{~nm}$ dicken Sensors. Die Feldwerte sind in zwei verschiedenen Höhen berechnet.

feld kann dieser Effekt ebenfalls erheblich reduziert, beziehungsweise komplett eliminiert, werden. Dennoch zeigt das Beispiel FePt, dass bei der Auswahl der Sensoren die dargestellte Limitierung sorgsam beachtet werden muss. Aus diesem Hintergrund ist gerade bei der Verwendung sehr dünner Indikatoren, mit denen man experimentelle Auflösungen nahe der theoretischen erreichen möchte, die Kenntnis der von den Strukturen erzeugten Streufelder in der Höhe der aktiven Schicht im Vorfeld der Untersuchungen (durch Simulationen) sinnvoll, um eine umfassende Streufelddetektion mit Hilfe geeigneter Sensoren zu gewährleisten.

\subsection{Rekonstruktion in der Ebene ausgerichteter Magnetisierungsverteilungen}

Wie in Kapitel 2 dargelegt, muss im Fall einer reinen inplane-Magnetisierung zunächst die Volumenladungsdichte $\rho_{\|}$betrachtet werden. Durch das Auftreten des zusätzlichen Faktors $1 / \mathrm{k}$ in Gl. 2.20 stellen sich die entsprechenden Transferfunktionen qualitativ anders dar. Für Magnetisierungsverteilungen der Gestalt $\vec{M}_{\|}(\vec{k})=M_{x}(\vec{k}) \vec{e}_{x}+M_{y}(\vec{k}) \vec{e}_{y}$ ergibt sich mit $\sigma_{\perp}(\vec{k})=0$ für die inplane-Volumenladungsdichteverteilung 


$$
\rho_{\|}(\vec{k})=\frac{H_{z}(\vec{k}, z)}{\alpha\left(k, d_{\text {Film }}, z\right)}
$$

mit der Feldtransferfunktion nach Gleichung 2.19. Im Unterschied zu einer reinen $M_{z^{-}}$ Verteilung kann bei Vorliegen einer beliebigen Magnetisierungsrichtung in der Ebene die $H_{z}$-Verteilung nicht direkt in den Komponentenvektor $\left(M_{x}, M_{y}\right)$ umgerechnet werden. Dies ist erst unter Berücksichtigung einer zusätzlichen Randbedingung möglich.

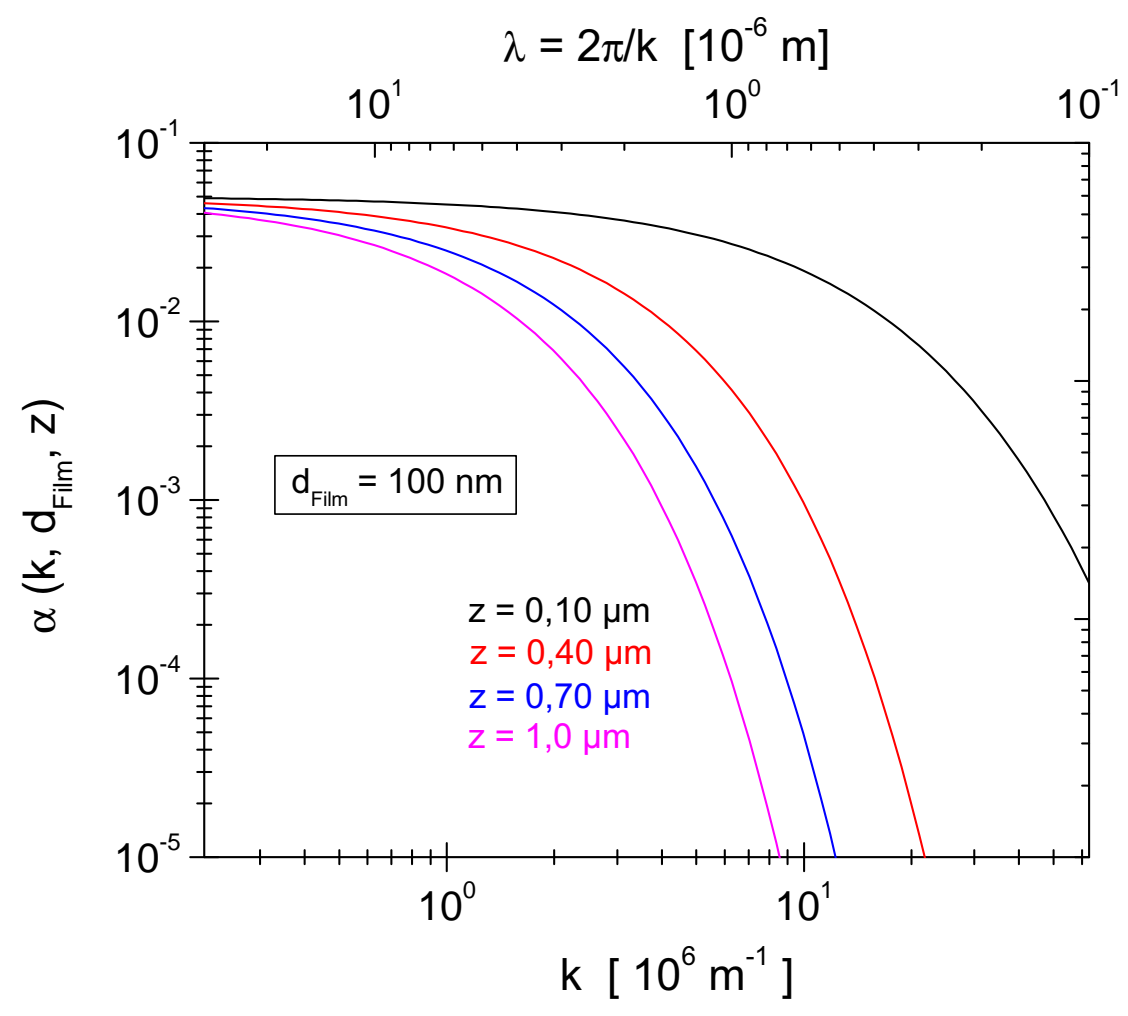

Abbildung 6.12: Inplane Feldtransferfunktion eines 100 nm dicken magnetischen Körpers in Abhängigkeit der räumlichen Frequenz, bzw. Wellenlänge, für verschiedene Messhöhen.

Abbildung 6.12 zeigt die nach Gl. 2.19 berechnete Feldtransferfunktion für verschiedene Messhöhen z bei fester Filmdicke von $d_{\text {Film }}=100 \mathrm{~nm}$. Diese wird im Folgenden wieder konstant gelassen und entspricht dem Beispiel der $\mathrm{PrCo}_{5}$-Filme. Man erkennt, dass $\alpha\left(k, d_{\text {Film }}, z\right)$ bereits für kleinere k-Werte als im senkrechten Fall deutlich reduziert ist, was für die Messbarkeit solcher inplane-Streufeldverteilungen eine immer bessere Sensitivität bzw. eine niedrigere Messhöhe erforderlich macht.

Es wird nun analog zum Fall senkrechter Magnetisierungsverteilungen die Sensortransferfunktion und anschließend die Abbildungstransferfunktion für den inplane-Fall berechnet. Danach ergibt sich für das gemittelte Streufeld (gemäß Gl. 2.19 und 6.6)

$$
\bar{H}_{z}(k)=\frac{1}{2 d_{M O L}} \frac{e^{\frac{k d_{F i l m}}{2}}-e^{-\frac{k d_{F i l m}}{2}}}{k^{2}} e^{-k d_{0}}\left(1-e^{-k d_{M O L}}\right) \cdot \rho_{\|}(\vec{k})=\beta_{i n}(k) \cdot \rho_{\|}(\vec{k})
$$


mit der inplane-Sensortransferfunktion $\beta_{\text {in }}(k)$, aus der man durch Multiplikation mit der Gaußfunktion (vgl. Gl. 6.9) im Fourierraum wieder die inplane-Abbildungs- transferfunktion $\gamma_{i n}(k, \sigma)$ erhält

$$
\bar{H}_{z}(k)=\beta_{i n}(k) e^{-\frac{k^{2} \sigma^{2}}{2}} \cdot \rho_{\|}(\vec{k})=\gamma_{i n}(k, \sigma) \cdot \rho_{\|}(\vec{k}) .
$$

Der Verlauf dieser beiden Funktionen ist mit den gleichen Parametern des senkrechten Falls (Abb. 6.3) in Abbildung 6.13 dargestellt.

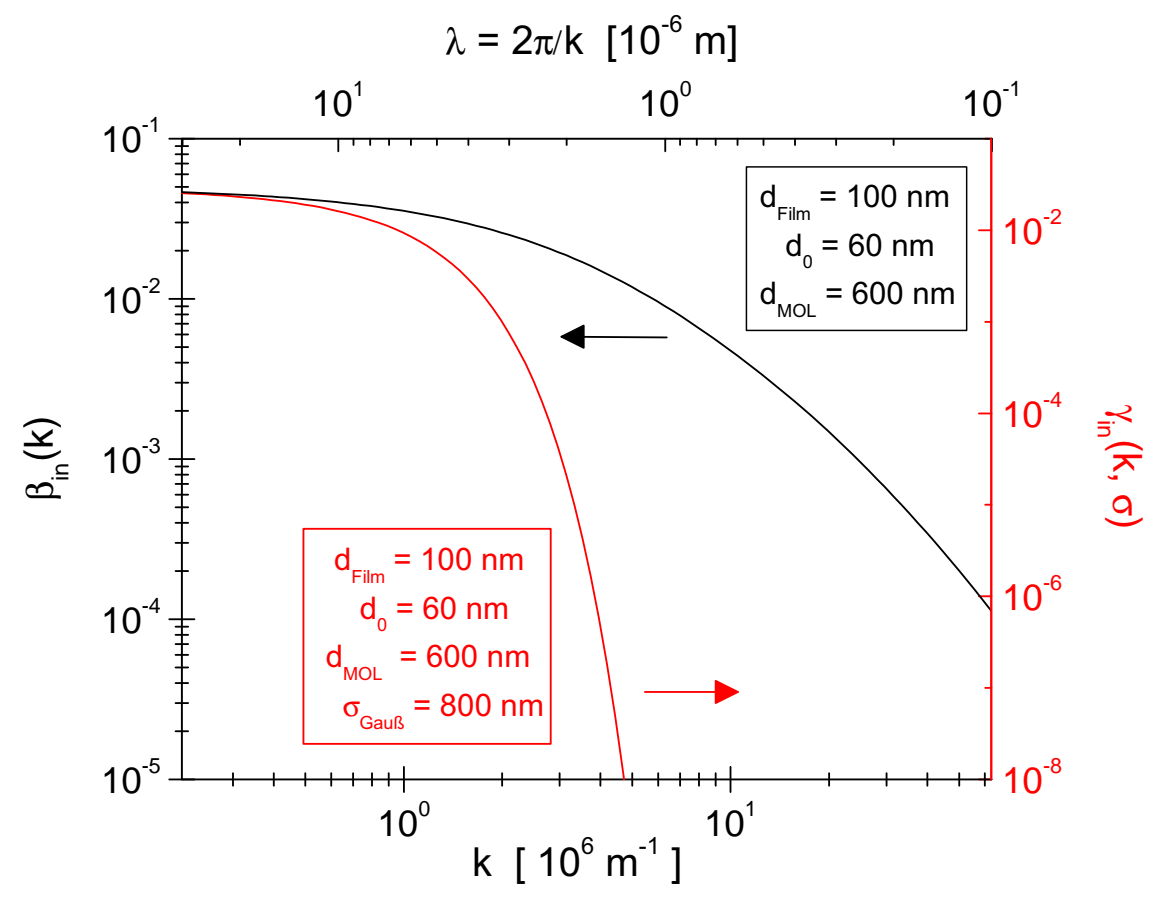

Abbildung 6.13: Verläufe der inplane Sensor- (linke Ordinate) und inplane Abbildungstransferfunktion (rechte Ordinate) in Abhängigkeit der räumlichen Frequenz, bzw. Wellenlänge. Die Simulationsparameter sind in den farblich gekennzeichneten Kästchen angegeben.

Man erkennt beim Vergleich der Sensortransferfunktion mit den Feldtransferfunktionen aus Abb. 6.12, dass $\beta_{\text {in }}$ bis zu einer Wellenlänge von etwa $2 \mu \mathrm{m}$ im Wesentlichen dem Verlauf von $\alpha$ bei einer mittleren Messhöhe von $400 \mathrm{~nm}$ folgt. In diesem Bereich entspricht das höhengemittelte Streufeld also noch weitgehend einem homogen über die gesamte Sensorschichtdicke gemittelten Feld. Für kleinere Wellenlängen wird $\beta_{i n}$ immer stärker durch die Feldtransferfunktion bei kleinen Messhöhen bestimmt. Für die Streufeldabbildung bei größeren räumlichen Frequenzen resultiert das Signal damit immer weniger vom gesamten Sensor her, sondern vielmehr von den untersten, der magnetischen Struktur nahen Lagen der magneto-optisch aktiven Schicht.

Nach der Multiplikation der Sensortransferfunktion mit der Gaußfunktion im Frequenzraum ergibt sich ein nahezu identisches Bild wie im senkrechten Fall. $\gamma_{i n}$ bricht für Wellenlängen kleiner als $2 \mu m$ stark ein.

Abbildung 6.14 zeigt die mittels inplane-Abbildungstransferfunktion rekonstruierte Volumenladungsdichteverteilung an dem Beispiel einer $10 \mu m \times 10 \mu m$ PrCo $_{5}$-Struktur (Kapi- 


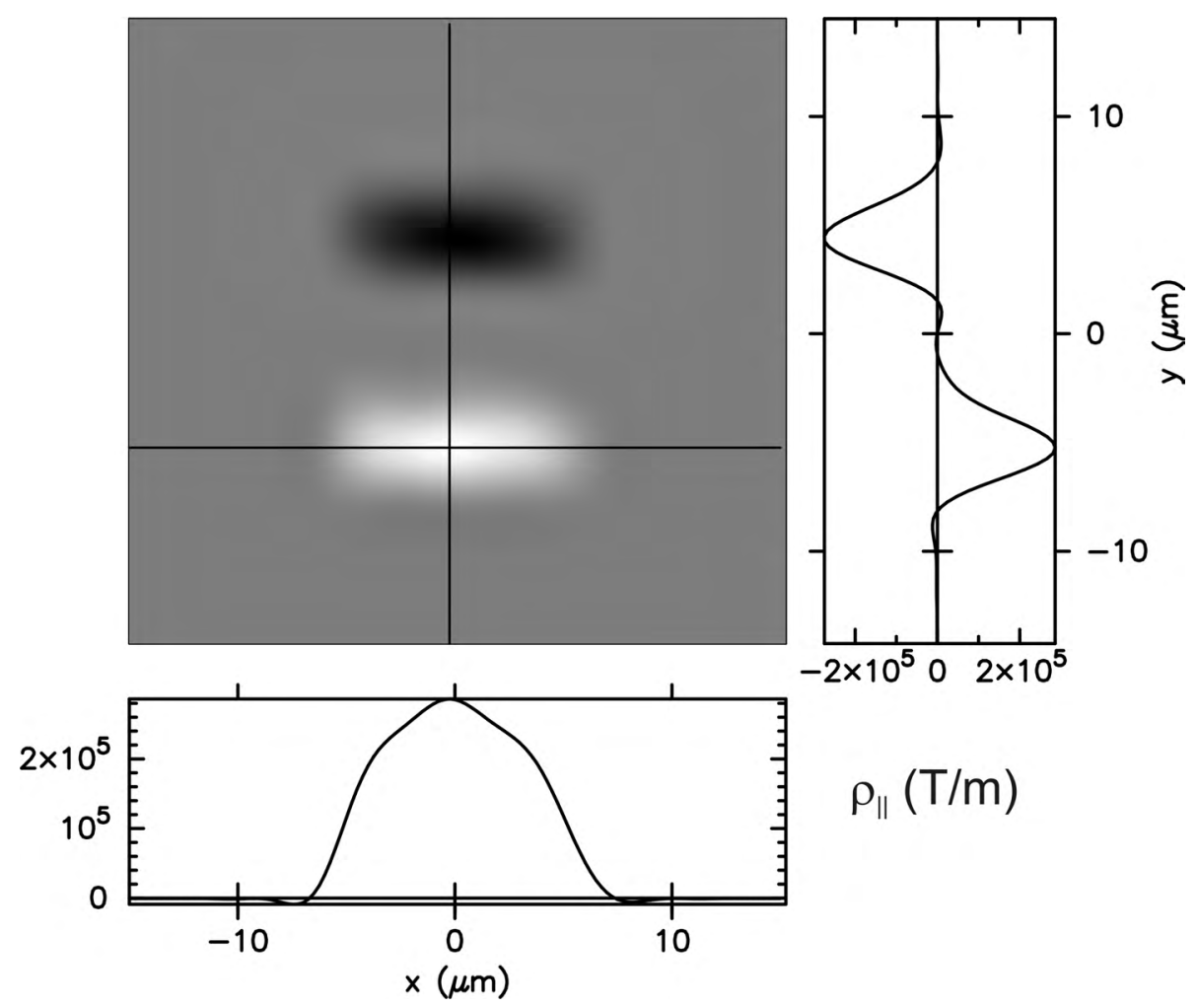

Abbildung 6.14: Experimentell bestimmte Volumenladungsdichteverteilung für eine $10 \mu m \times$ $10 \mu \mathrm{m} \mathrm{PrCo}_{5}$-Strukturnach Anwendung der inplane-Abbildungstransferfunktion. Die y-Richtung des Koordinatensystems zeigt in die kristallographische [110]-Richtung.

tel 5.6) mit einer Magnetisierung in [110]-Richtung. Auffällig ist hierbei die ausgeprägte Breite der Ladungsdichteverteilung an den Rändern. Dies ist eine direkte Konsequenz der Filterung der experimentell ermittelten Streufeldverteilung, die durch das Abschneiden der höheren Frequenzen effektiv das räumliche Auflösungsvermögen begrenzt (vgl. voriger Abschnitt).

Wird der Inversionsformalismus analog dem senkrechten Fall auf theoretisch berechnete Streufeldverteilungen einer Struktur mit inplane-Magnetisierung angewendet, so zeigt sich, dass die Breite der Ladungsverteilung praktisch identisch ist mit der durch die Abschneidefrequenz der Filterung gegebenen räumlichen Auflösung (siehe Abb. 6.15). Für die experimentell ermittelten Daten (Abb. 6.14) beträgt die Halbwertsbreite der Ladungsverteilung z.B. 2,73 $\mu \mathrm{m}$, das Auflösungsvermögen liegt bei 2, $5 \mu \mathrm{m}$.

Die eigentliche Inversionsrechnung ist nach Kenntnis von $\rho_{\|}$abgeschlossen, so dass die einzelnen Magnetisierungskomponenten nun im Ortsraum bestimmt werden können.

\section{Bestimmung der inplane-Magnetisierungskomponenten}

Die Rekonstruktion der beiden Vektorkomponenten $M_{x}(x, y)$ und $M_{y}(x, y)$ aus dem Skalarfeld $\rho_{\|}(x, y)$ erfolgt nun unter Verwendung der Sprungrelationen. Um dies durchführen zu können, wird das magnetische Medium in leichter zu beschreibende Struktureinheiten aufgeteilt. Mikromagnetische Modelle verwenden dafür häufig eine sogenannte Voronoi- 


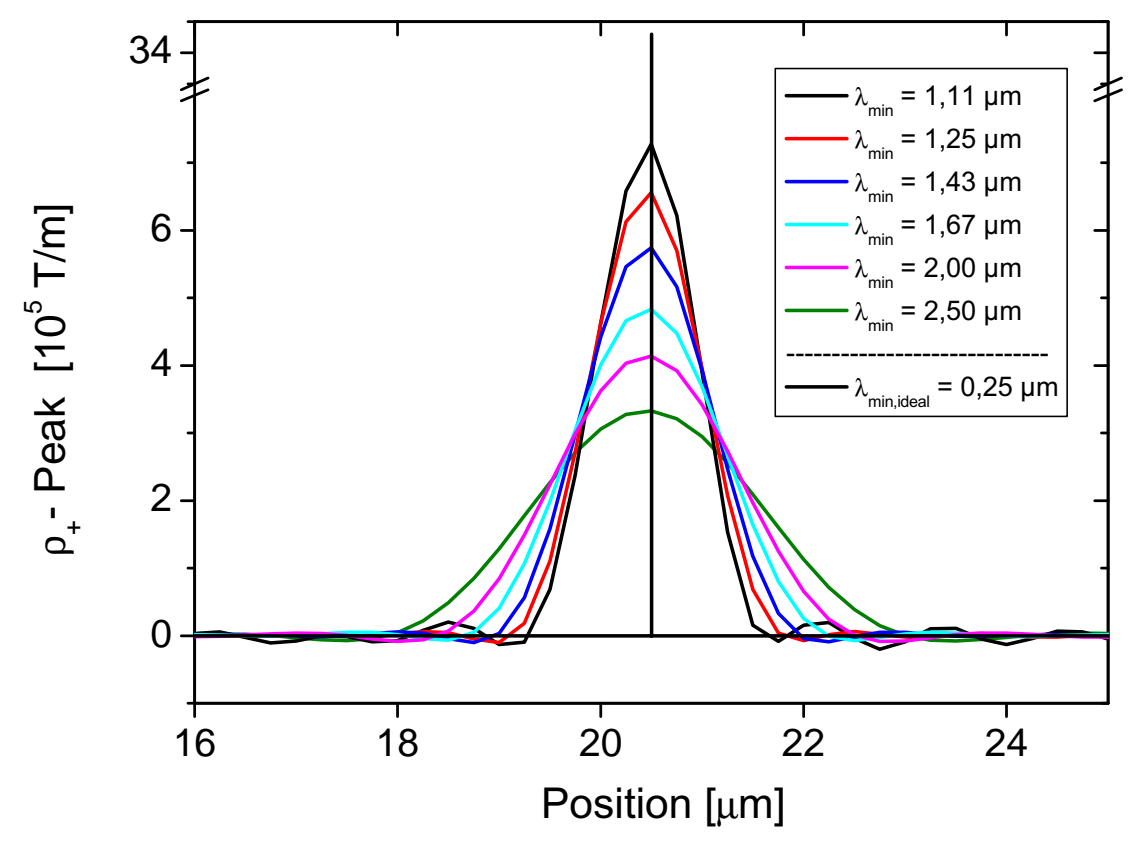

Abbildung 6.15: Entwicklung des positiven Ladungsdichtemaximums einer inplane ausgerichteten Magnetisierungsverteilung in Abhängigkeit der Ortsauflösung, bestimmt nach Rekonstruktion aus theoretisch berechneten Streufeldverteilungen. Der $\delta$-förmige Peak ergibt sich bei idealer Rekonstruktion und hat die Breite eines Pixels.

Tesselierung [122, 123], die das dünne magnetische Medium in eine, das gesamte Volumen ausfüllende, periodische Kornstruktur zerlegt. Diese Körner stellen Bereiche mit konstantem, einheitlichen Magnetisierungsvektor dar, die durch infinitesimal dünne Domänenwände oder durch die Oberfläche der Struktur begrenzt sind. Miles et al. [124, 125] konnten dieses Modell noch erweitern, in dem sie ein Gefüge aus Körnern beliebiger Form und Anordnung zuließen und damit unphysikalische Anisotropien, die sich aus der Periodizität der Anordnung und Form der Körner ergaben, vermieden. Dieses Modell wird u. a. bei der Simulation von magnetischen Speichermedien verwendet und zeigt für berechnete Ummagnetisierungskurven eine gute Übereinstimmung mit experimentellen Daten. Die mittlere Korngröße kann dem tatsächlichen Kornwachstum angepasst werden.

Das Zugrundelegen einer solchen magnetischen Mikrostruktur für die in dieser Arbeit verwendeten hartmagnetischen $\mathrm{PrCO}_{5}$-Filme ist aufgrund ihrer sehr hohen kristallinen Anistropie ebenfalls sinnvoll. Die Konsequenz eines solchen Magnetisierungszustandes ist, dass sich nun die inplane-Volumenladungsdichte $\rho_{\|}$zu einer reinen Oberflächenladungsdichteverteilung $\sigma_{\|}$auf den Rändern dieser Körner reduziert. Als Analogon zu dem in Kapitel 2 bereits gezeigten senkrechten Fall ergibt sich im idealen Fall damit $\rho_{\|}=\sigma_{0} \cdot \delta(x)$ bzw. $\rho_{\|}=\sigma_{0} \cdot \delta(y)$ (bzw. auch Kombination aus beiden Anteilen). Die Sprungrelation für den Potentialanteil der inplane-Magnetisierung lautet damit

$$
\sigma_{\|}(x, y)=\vec{n}_{\|}(x, y) \cdot \vec{M}_{\|}(x, y)=M_{x}(x, y) \vec{n}_{\|}(x, y) \cdot \vec{e}_{x}+M_{y}(x, y) \vec{n}_{\|}(x, y) \cdot \vec{e}_{y}
$$

$\vec{n}_{\|}(x, y)$ bezeichnet im Weiteren den inplane-Normalenvektor in der Ebene der zu untersuchenden Strukturen. Treten beide Komponenten auf, so ergibt sich die notwendige 
zusätzliche Information über die Richtung von $\vec{n}_{\|}(x, y)$ direkt aus der Ladungsverteilung gemäß

$$
\vec{n}_{\|}(x, y)=\frac{\vec{\nabla}_{\|} \rho_{\|}(x, y)}{\left|\vec{\nabla}_{\|} \rho_{\|}(x, y)\right|} .
$$

Für diskrete Verteilungen nimmt Gl. 6.14 die Form $\vec{n}_{\|}=\left(\vec{\nabla}_{\|} \rho_{\|} \cdot p\right) /\left|\rho_{\|}\right|$an. Ein Problem für die explizite Magnetisierungsbestimmung aus der Ladungsdichteverteilung wie sie sich in den Abb. 6.14 und Abb. 6.15 darstellen, folgt aus der begrenzten Auflösung der Messmethode nach der Fourierfilterung. In den experimentellen Bildern zeigt sich die Volumenladungsdichte vielmehr in der Form $\rho_{\|}(x)=\sigma_{0} \delta(x) * G(x)$, d.h. als Faltung mit der Aufösungsfunktion. Die Integration der Ladungsdichte liefert aber einen quantitativ korrekten Wert

$$
\sigma_{0}=\int_{-\infty}^{\infty} \rho_{\|}(x) d x
$$

Die ideale Darstellung über eine Deltafunktion, die sich aus der Tesselierung gemäss Gl. 6.13 ergibt, wird insofern nicht exakt umgesetzt. Bei Anwendung der obigen Berechnungsweise auf die $10 \times 10 \mu^{2} \mathrm{PrCo}_{5}$-Struktur, erhält man als Gesamtmagnetisierung in [110]Richtung die in Abbildung 6.16(a) dargestellte Verteilung. Der Wert für die Remanenzmagnetisierung von $\mu_{0} M_{110}(x, y)=0,88 \mathrm{~T}$ steht in ausgezeichneter Übereinstimmung mit den SQUID-Messungen an diesem Film und bestätigt damit, dass durch die Integration der Ladungsdichteverteilung ein korrekter Wert für die Magnetisierung bestimmt wird.

a.)

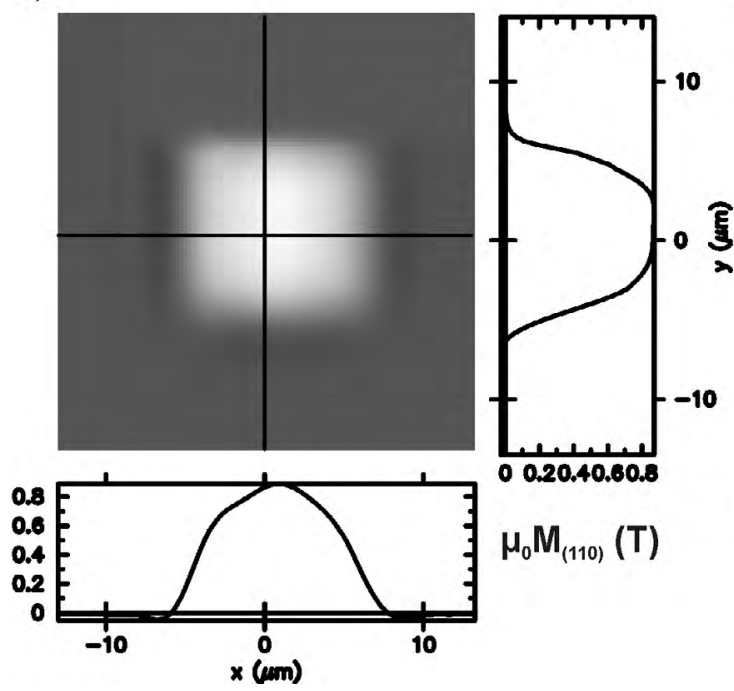

b.)

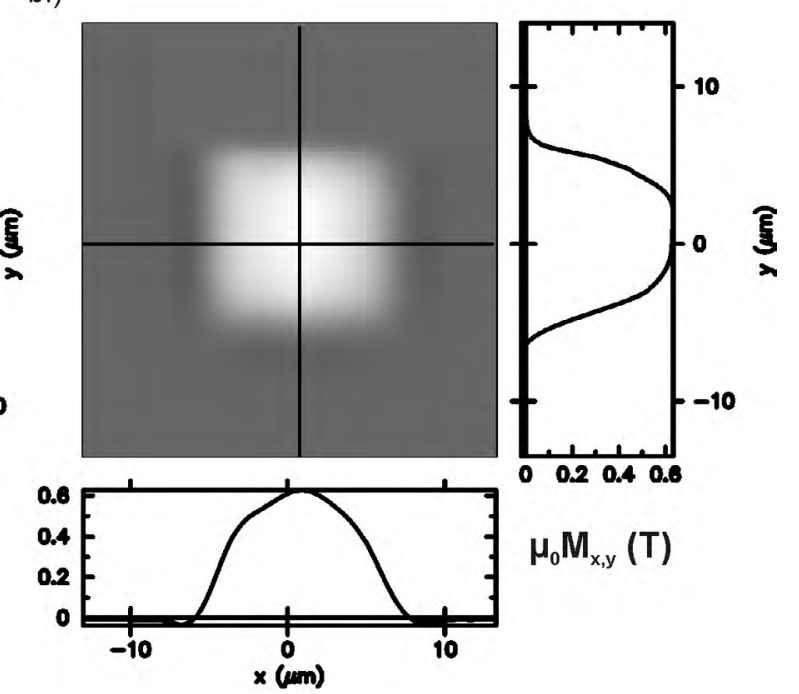

Abbildung 6.16: (a) Experimentell rekonstruierte Gesamtmagnetisierung in [110]-Richtung der $10 \mu \mathrm{m} \times 10 \mu \mathrm{m} \mathrm{PrCo}_{5}$-Struktur aus der Ladungsdichteverteilung in Abb. 6.14. (b) Aus (a) bestimmte Magnetisierungskomponente $\mu_{0} M_{y}$ durch Zurückrechnen auf die leichten Richtungen der Magnetisierung des $\mathrm{PrC}_{5}$ auf $\mathrm{MgO}(100)$, die unter einem Winkel von $45^{\circ}$ entlang der Substratkanten liegen (siehe Kap. 4.4). Bei entsprechender Drehung des Koordinatensystems um $90^{\circ}$, erhält man $\mu_{0} M_{x}$

Bei Vergleich mit der Ladungsdichte erkennt man, dass die Magnetisierung den $\rho$-Verlauf widerspiegelt. In y-Richtung ist das Profil an den Kanten entsprechend etwa der doppelten 
Halbwertsbreite der Ladungsdichteprofile ausgeschmiert, im Innern ist die Magnetisierung konstant. Entlang der x-Richtung zeigt sich ein etwas gewölbter Verlauf, wie er auch in den Ladungsdichteprofilen in dieser Richtung auftritt. Diese charakteristische Verteilung ist eine direkte Konsequenz der begrenzten Auflösung und der entsprechenden Integration über die verschmierte Ladungsdichte. Während in y-Richtung zwei $\delta$-förmige Peaks mit der Gaußkurve gefaltet werden, findet dies in x-Richtung mit einer Rechteckfunktion statt. $\mathrm{Zu}$ ergänzen ist an dieser Stelle, dass niederfrequentes Rauschen, das nicht herausgefiltert wird, im Außenraum der Struktur zu Artefakten führt, so dass dieser nachträglich auf Null gesetzt wird. In Abbildung 6.16(b) wurde die Gesamtmagnetisierung in die $\mu_{0} M_{y}(x, y)$ Komponente umgerechnet. Hierbei wurde lediglich die Tatsache verwendet, dass nur an zwei gegenüberliegenden Kanten der Struktur Ladungen auftreten und diese Kanten unter einem Winkel von $45^{\circ} \mathrm{zu}$ den leichten Achsen der Magnetisierung in dem $\mathrm{PrCo}_{5}$-System liegen. Die Komponente kann also durch Multiplikation mit $\cos 45^{\circ}$ berechnet werden und liegt mit $\mu_{0} M_{y}(x, y)=0,63 \mathrm{~T}$ wie erwartet sehr nahe dem Wert, der auch in den SQUIDMessungen in y-Richtung gemessen wird. Bei Drehung des Koordinatensystems um $90^{\circ}$, würde man analog die $\mu_{0} M_{x}(x, y)$-Verteilung erhalten.

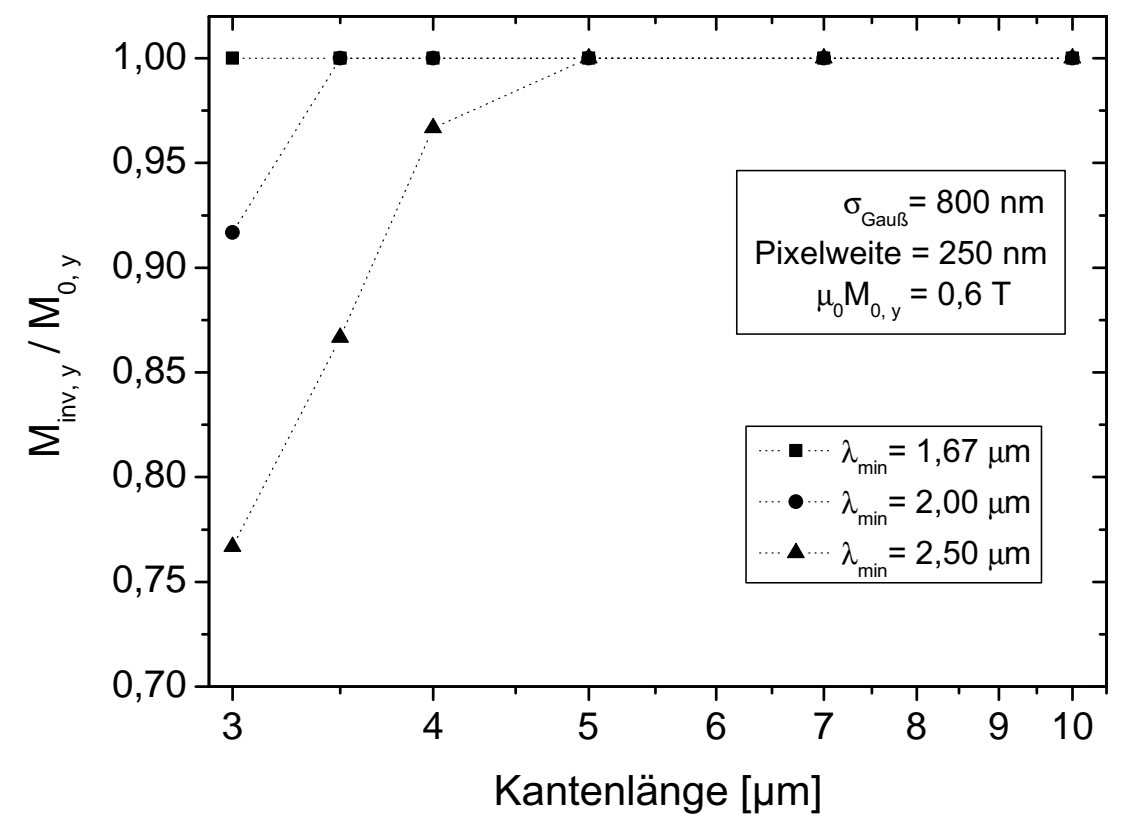

Abbildung 6.17: Verhältnis aus rekonstruierter zu Referenzmagnetisierung als Funktion der Kantenlänge der simulierten Quadrate bei fester inplane Magnetisierung in y-Richtung und verschiedenen Ortsauflösungen.

Für den Fall reiner inplane Magnetisierung kann man nun auch wieder durch den Vergleich mit theoretisch berechneten Streufeldverläufen und deren anschließender Inversion testen, bei welcher Ortsauflösung noch Quadrate vorgegebener Kantenlänge vollständig rekonstruiert werden können. Analog zum senkrechten Fall ist in Abbildung 6.17 das Verhältnis aus invertierter $M_{i n v, y}$ zu Referenzmagnetisierung $M_{0, y}$ gegen die Kantenlänge der Quadrate aufgetragen. Als Simulations- bzw. Korrekturparameter für die inplane Abbildungstransferfunktion wurden die Standardwerte verwendet. Es zeigt sich, dass analog der senkrechten Magnetisierung ebenfalls Kantenlängen bis zu $5 \mu m$ bei allen Auflösungen 
quantitativ korrekt invertiert werden. Bei der kleinsten Ortsauflösung wird die Magnetisierung sogar bei allen verwendeten Strukturgrößen vollständig rekonstruiert. Die Grenze ist durch das Überlappen der beiden Ladungsdichten an den Kanten gegeben. Reduziert sich die Fläche unter den Maxima aufgrund der begrenzten Auflösung, so liefert auch die Integration der Ladungen ein zu kleines Signal.

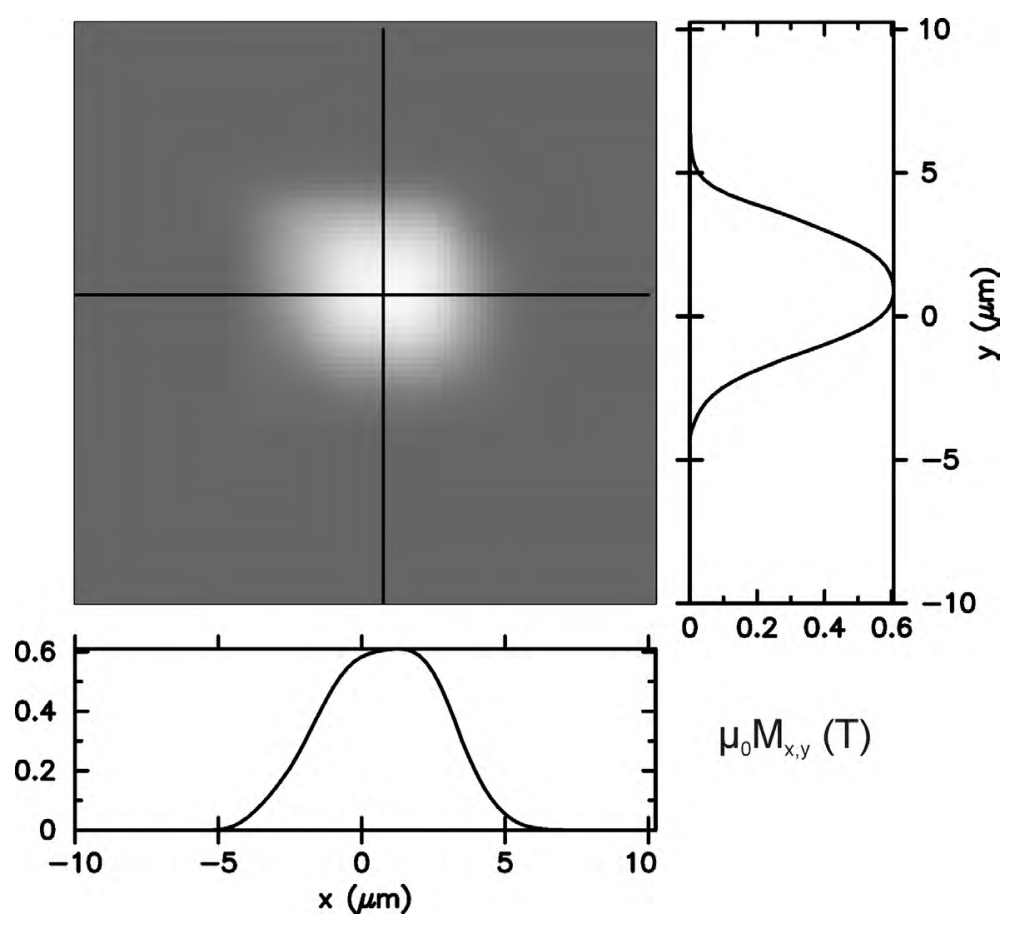

Abbildung 6.18: Experimentelle Rekonstruktion der y-Magnetisierungskomponente eines $5 \times$ $5 \mu \mathrm{m}^{2}$ Quadrates unter denselben Voraussetzungen wie in Abb. 6.16.

Dieses Ergebnis kann experimentell an einer $5 \times 5 \mu m^{2}$ großen $\mathrm{PrCo}_{5}$-Struktur verifiziert werden. Abbildung 6.18 zeigt die Remanenzmagnetisierung in y-Richtung der Struktur. Die Ortsauflösung beträgt $\lambda_{\min }=2,5 \mu \mathrm{m}$. Die Remanenz ergibt sich nach Inversion zu $\mu_{0} M_{y}(x, y)=0,61$ T. Damit liegt der Wert lediglich 3\% unter dem Referenzwert aus der SQUID-Messung. Eine Rekonstruktion der Magnetisierung an noch kleineren Strukturen konnte experimentell nicht mehr durchgeführt werden, da sich Strukturen mit einer Kantenlänge von $2 \mu \mathrm{m}$ nicht mehr magneto-optisch abbilden ließen. 


\section{Anfangsstadien der Ummagnetisierung hartmagnetischer CoPt-Strukturen}

Die magneto-optische Messmethode bietet die Möglichkeit große Felder von Strukturen unterschiedlicher Form und Größe in einer Aufnahme quantitativ zu analysieren. Dies erlaubt die Untersuchung großer, regelmäßiger Anordnungen magnetischer Elemente, die beispielsweise über ihre Streufelder wechselwirken und so eine zusätzliche Konfigurationsanisotropie zeigen können. In solchen Systemen, die aufgrund der Anordnung vieler Einzelstrukturen über große Längenskalen ausgedehnt sind, bietet die Möglichkeit der quantitativen Bestimmung von Streufeldern und Wechselwirkungspotentialen in diesem Längenskalenbereich einen effektiven Zugang zum Verständnis der charakteristischen Eigenschaften.

Während kollektive Effekte in eher weichmagnetischen Strukturen auftreten [126, 127], ist das experimentelle Ummagnetisierungsverhalten von Hartmagneten maßgeblich durch die Defektstrukturen des zugrundeliegenden Films bestimmt. Wie sich im Folgenden zeigt, kann es im Falle eines Ummagnetisierungsmechanismus, der auf der Nukleation und dem Wachstum von Gegendomänen basiert, z.B. durch das Auftreten charakteristischer Keimbildungszentren und deren räumlicher Verteilung, zu einem sehr inhomogenen Verhalten einer Anordnung bezüglich der Ummagnetisierung einzelner Elemente kommen. Diese Inhomogenitäten haben zur Konsequenz, dass sehr große Anordnungen immer als Ganzes analysiert werden müssen, da es keine repräsentativen Einzelobjekte gibt, was wiederum große Messauschnitte bedingt. Die Nukleation, d.h. das Frühstadium solcher Prozesse, spielt dabei eine wesentliche Rolle.

Um kollektive Effekte in z.B. magnetisch moderat harten Systemen sinnvoll analysieren zu können, sollten die Auswirkungen der Mikrostruktur auf die Ummagnetisierungscharakteristiken künstlich, lateral strukturierter Filme zunächst in nicht wechselwirkenden Systemen verstanden sein. Dazu werden im folgenden Strukturen von Hartmagneten untersucht, deren Abmessungen und Abstände $(\geq 1 \mu \mathrm{m})$ so groß sind, dass Wechselwirkungseffekte zu vernachlässigen sind. Dabei steht die Frage im Vordergrund, ob ein Zusammenhang zwischen dem Ummagnetisierungsverhalten und der Strukturgröße bzw. auch der geometrischen Form der Elemente, unter Berücksichtigung der Defektverteilung, besteht. CoPt-Strukturen, die aufgrund ihrer hohen Remanenzmagnetisierungen klar definierte Streufelder produzieren, dienen dafür als Modellsystem.

Um die quantitativen Aussagen im Folgenden mit der auf Längenskalen unterhalb der Auflösungsgrenze der MOIF-Methode liegenden magnetischen Substruktur korrelieren zu können, wird mittels magnetischer Kraftmikroskopie diese komplementäre Information 
bestimmt und in einem qualitativen Vergleich herangezogen. Das Kapitel gliedert sich in eine sehr kurze Einführung in die relevanten Größen bei der Ummagnetisierung uniaxialer Hartmagnete, den anschließenden experimentellen Untersuchungen sowie einer Diskussion der Ergebnisse.

\subsection{Ummagnetisierung uniaxialer Hartmagnete}

Die treibende Kraft für die Ummagnetisierung eines magnetischen Körpers ist die Reduktion der Gesamtenergiedichte, wobei in den verwendeten uniaxialen Hartmagneten, wie es CoPt in der geordneten Phase darstellt, neben der magnetokristallinen Anisotropienergie die magnetostatische Energie einen wichtigen Beitrag liefert. Diese setzt sich aus der magnetostatischen Selbstenergie (Gl. 7.1) und aus der Zeeman-Energie (Gl. 7.2) in externen Feldern zusammen

$$
\begin{aligned}
E_{d e m} & =-\frac{\mu_{0}}{2} \int_{V} \vec{M} \cdot \vec{H}_{d e m} d^{3} r, \\
E_{Z} & =-\mu_{0} \int_{V} \vec{M} \cdot \vec{H}_{e x t} d^{3} r
\end{aligned}
$$

mit $\vec{H}_{d e m}$ als dem inneren (ohne externes Feld) oder auch Entmagnetisierungsfeld eines Körpers. Für den Fall ellipsoidaler Körper ist $\vec{H}_{d e m}$

$$
H_{d e m, i}=-\sum N_{i, j} M_{j}
$$

mit den $N_{i, j}$ als Elementen des Entmagnetisierungstensors. Während die magnetokristalline Anisotropie für (001) orientierte CoPt-Filme (oder auch FePt) eine senkrechte Magnetisierung bedingt, favorisiert die Formanisotropie bei dünnen Filmen grundsätzlich eine Magnetisierung in der Ebene. Diese beiden Energiebeiträge sind somit wesentlich für die remanente Magnetisierungsverteilung in den Körpern verantwortlich. Die Reduktion der Zeeman-Energie, die die Magnetisierung in Richtung des äußeren Feldes auszurichten versucht, ist bei der Ummagnetisierung im Feld die entscheidende Größe.

Prinzipiell gibt es zwei Mechanismen, die bei Hartmagneten mit uniaxialer Anisotropie betrachtet werden [34, 83, 102]: (1) Eindomänige Teilchen, die meist in den beiden Grenzfällen vollständig verankerter oder freier Domänenwände dargestellt werden, was gleichbedeutend ist mit dem Ausrichten der Magnetisierung in die harte oder in die leichte Richtung der Magnetisierung. (2) Nukleation und Wachstum von Domänen in größeren, mehrdomänigen Teilchen.

Im ersten Fall tritt eine Ummagnetisierung des Teilchens entweder durch eine kohärente Rotation der Momente im äußeren Feld (kollektive Mode) oder auch in nichtgleichförmigen Moden wie z.B. durch curling oder buckling auf. Das Vorliegen eines eindomänigen Magnetisierungszustandes in Teilchen und damit des zu (1) dargestellten Ummagnetisierungsprozesses zeigt sich abhängig von den verschiedenen Anisotropieenergien eines Systems in Relation zu der Austauschkopplung [50]. Nach [128] liegt der kritische 
Radius für den eindomänigen Zustand einer CoPt-Struktur bei etwa $50 \mathrm{~nm}$. Die in dieser Arbeit präparierten Strukturen liegen aber alle um 1 bis 2 Größenordnungen darüber, so dass im Weiteren der Mechanismus der Nukleation und des Wachstums von Domänen als relevanter Ummagnetisierungsprozeß zum Verständnis des Verhaltens der CoPtStrukturen betrachtet werden muss.

Geht man zunächst von einkristallinen Körpern aus, so ist nach der Brownschen Formel 7.4 [129] das Nukleationsfeld für den Keimbildungsprozess von Gegendomänen gegeben durch

$$
H_{n}=\frac{2 K_{u}}{\mu_{0} M_{s}}-N M_{s}
$$

Der erste Summand steht für das Anisotropiefeld $H_{a}$, das benötigt wird, um die Magnetisierung in der harten Richtung vollständig auszurichten. Dieser Beitrag stellt also eine energetische Barriere für die Rotation der Momente von einer leichten Richtung in eine andere dar. Der zweite Summand ist das Entmagnetisierungsfeld im Innern der Probe, das die Minimierung der Oberflächenladungen zur Folge hat. Bei Anisotropiefeldern, die größer sind als $H_{\text {dem }}$ muss durch ein externes Gegenfeld der aus der Differenz resultierende Energiebeitrag zur Domänenbildung aufgewendet werden.

Die sehr hohen Anisotropiekonstanten für perfekt geordnetes CoPt oder FePt führen danach zu Werten für $H_{n}$ im Bereich $\mu_{0} H_{n} \approx 9-10 \mathrm{~T}$. Solch extrem hohe Felder werden in realen Proben aber aufgrund der mit Defekten assoziierten Mikrostruktur nicht beobachtet. Strukturelle Fehler als Bereiche reduzierter Kristallanisotropie wirken im Sinne einer heterogenen Keimbildung als Nukleationszentren mit einem deutlich reduzierten Nukleationsfeld.

Dem Prozess der Nukleation folgt das weitere Wachstum dieser Gegendomänen durch die Wanderung von Domänenwänden. Dabei beeinflussen vor allem auftretende Haftkräfte (pinning) die Wandverschiebung. Bei Hartmagnetika fungieren Kristalldefekte, die eine reduzierte magnetokristalline Anisotropie besitzen, als attraktive Haftzentren und behindern eine Ummagnetisierung. Besitzt im Laufe des Prozesses bei einem externen magnetischen Feld der Körper keine makrosokopische Magnetisierung mehr, so bezeichnet man das relevante Feld als sogenanntes Koerzitivfeld $H_{c}$. Die lokalen Verhältnisse zwischen den Haftkräften und Gradienten in der magnetostatischen Energie bestimmen maßgeblich die Domänenwandbeweglichkeit und sind damit wichtig für ein Verständnis des Zustandekommens der Koerzitivfelder (siehe z.B. [34, 83, 102]).

\subsection{Experimentelle Ergebnisse zum Ummagnetisierungsverhalten von CoPt-Strukturen unterschiedlicher Größe und Form}

Abbildung 7.1 zeigt zwei Übersichtsbilder der remanenten magnetischen Streufeldverteilung von Anordnungen, die aus CoPt-Strukturen verschiedener Größe und Form bestehen, die unterschiedlichen externen Magnetfeldern ausgesetzt wurden. Abbildung 7.1(a) gibt die remanente Streufeldverteilung nach Aufmagnetisieren in einem externen Feld 
a.)

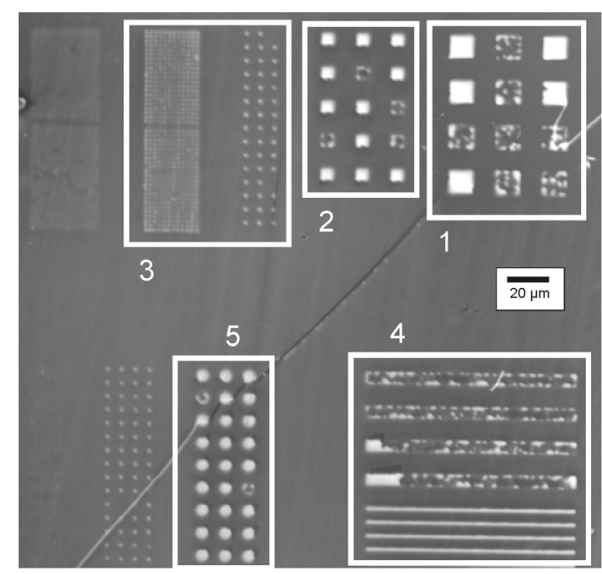

b.)

$H_{z, \text { ext }}=-100 m T$

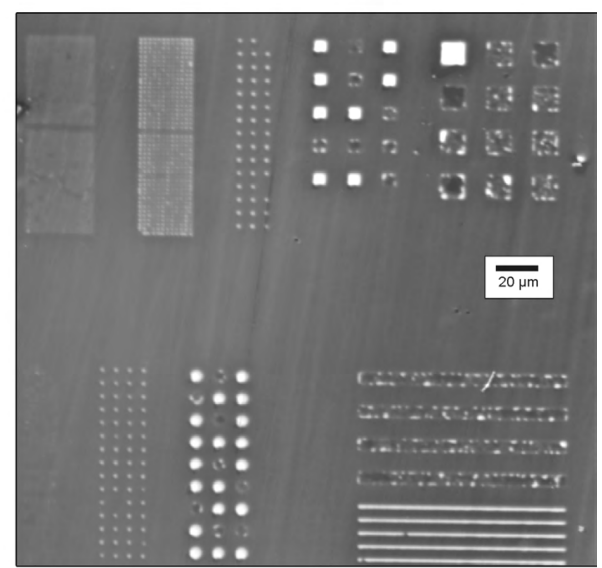

Abbildung 7.1: Remanente Streufeldverteilungen von CoPt-Strukturen nach (a) Aufmagnetisieren in einem externen Feld von $\mu_{0} H_{z, e x t}=+700 \mathrm{mT}$ und (b) nach Anlegen eines Gegenfeldes von $\mu_{0} H_{z, e x t}=-100 \mathrm{mT}$. Die weißen Kästchen umrahmen die Strukturfelder, die später detaillierter untersucht werden, wobei die Strukturgrößen entsprechend (1) $10 \times 10 \mu \mathrm{m}^{2}$, (2) $5 \times 5 \mu \mathrm{m}^{2}$ (3) $1 \times 1 \mu m^{2}$ mit $5 \mu \mathrm{m}$ bzw. $1 \mu \mathrm{m}$ Abstand, (4) $5 \times 80 \mu m^{2}$ bzw. $1 \times 80 \mu m^{2}$ und (5) $d=5 \mu \mathrm{m}$ sind.

von $\mu_{0} H_{z, \text { ext }}=+700 \mathrm{mT}$ wieder. Abb. 7.1(b) zeigt die Strukturfelder nach dem Anlegen eines negativen Gegenfeldes von $\mu_{0} H_{z, e x t}=-100 \mathrm{mT}$. Rein qualitativ ist erkennbar, dass beispielsweise bei variierender Strukturgröße unterschiedlich viele Strukturen ihren Magnetisierungszustand geändert haben. Mittels des Rekonstruktionsschemas aus dem vorangegangenen Kapitel kann man diese remanenten Magnetisierungsverteilungen und deren Änderungen lokal quantitativ bestimmen.

Im folgenden soll zunächst am Beispiel der Quadrate mit $5 \mu m$ Kantenlänge eine ausführlichere Beschreibung des Ummagnetisierungsverhaltens der Anordnung gegeben werden, bevor später vergleichend auf die Unterschiede in den Strukturfeldern anderer Größe und Form eingegangen wird.
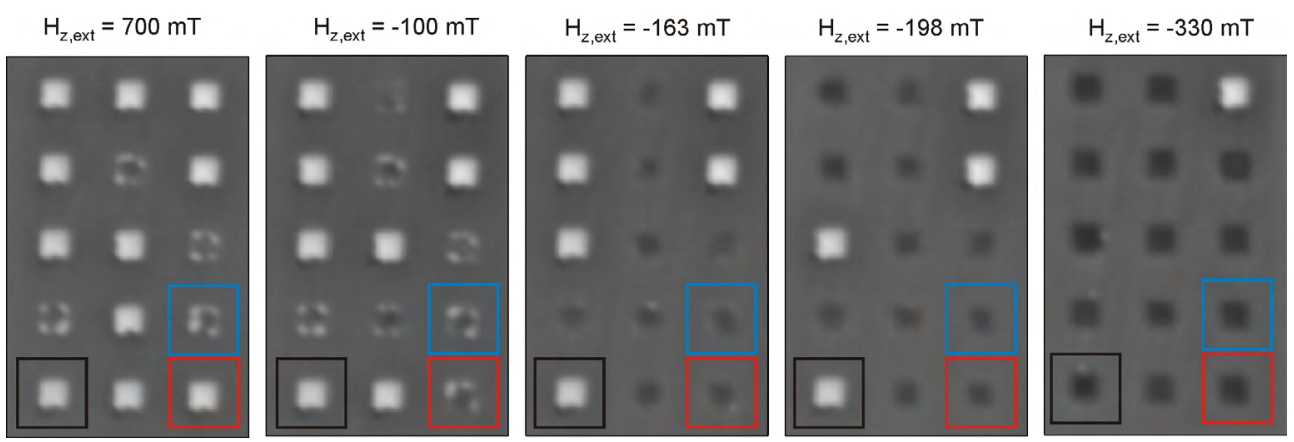

Abbildung 7.2: Anfangstadien des Ummagnetisierungsprozesses von $5 \times 5 \mu m^{2}$ großen CoPtStrukturen. Gezeigt sind die remanenten Streufeldverteilungen nach Anlegen der über den Bildern angegebenen Gegenfeldern (Bild 2-5).

Abbildung 7.2 zeigt die Ausschnittsvergrößerung der Anordnung von $5 \times 5 \mu m^{2}$ großen CoPt-Strukturen, die einen nicht vollständig abgeschlossenen Ummagnetisierungsprozess 
durchlaufen. An den beispielhaft umrandeten Quadraten erkennt man, dass nach dem Aufmagnetisieren sowohl Elemente mit domänenhafter Substruktur als auch im eindomänigen Zustand auftreten. Legt man nun sukzessiv steigende negative Ummagnetisierungsfelder an, so lässt sich eine Änderung des mittleren Streufeldkontrastes der einzelnen Quadrate erkennen. Es zeigt sich, dass der Ummagnetisierungprozess für die einzelnen Quadrate lokal sehr unterschiedlich verläuft und man nicht selbstverständlich auf ein universelles, repräsentatives Verhalten aller Strukturen schließen kann. Bei der quantitativen Berechnung der gemittelten Magnetisierung der markierten Quadrate wird dies deutlicher.

Wendet man das Rekonstruktionschema nun auf die obigen Strukturen an, so erhält man am Beispiel der remanenten Magnetisierung nach Aufmagnetisieren die in Abbildung 7.3 dargestellte Verteilung. Bei Verwendung der in der Bildunterschrift angegebenen Rekonstruktionsparameter zeigt sich zunächst wiederum eine sehr gute Übereinstimmung mit den aus der SQUID-Messung zu erwartenden Werten für die Sättigungsmagnetisierung von $\mu_{0} M_{s}=1 \mathrm{~T}$, wie der Vergleich mit den vollständig aufmagnetisierten Quadraten bestätigt (vgl. siehe Abb. 7.9).

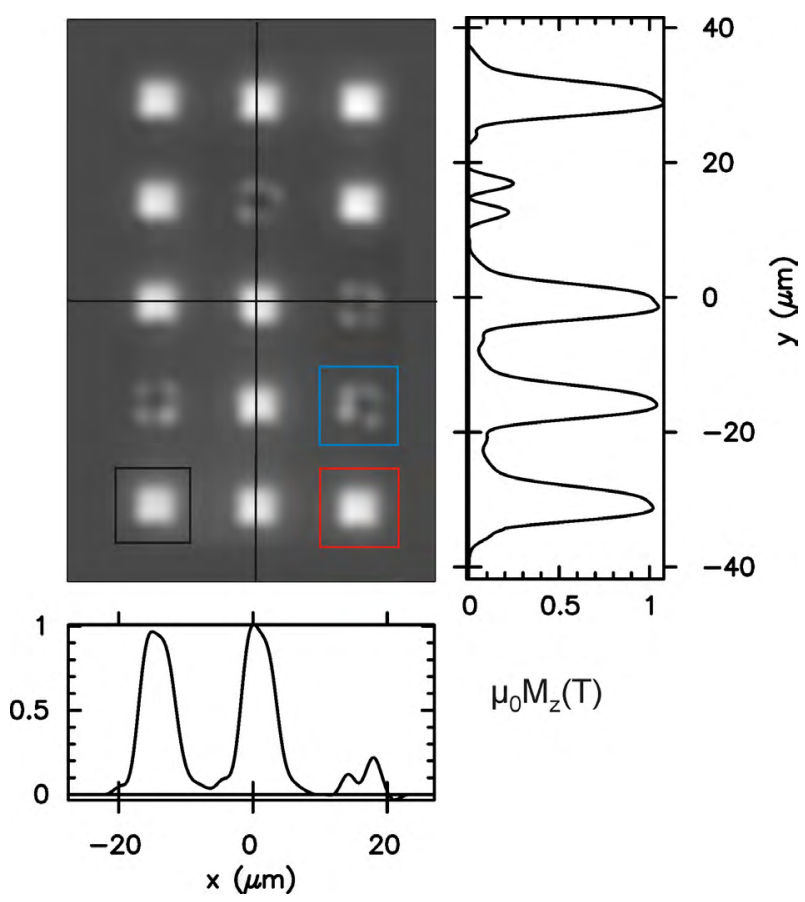

Abbildung 7.3: Remanente Magnetisierungsverteilung der in Abb. 7.2 (Bild 1) gezeigten $5 \times$ $5 \mu \mathrm{m}^{2}$ großen CoPt-Strukturen nach Aufmagnetisieren im Feld mit $\mu_{0} H_{z, e x t}=+700 \mathrm{mT}$. Der verwendete Sensor ist $1 \mu \mathrm{m}$ dick, und die experimentelle Auflösung beträgt ebenfalls etwa $\sigma \approx$ $1 \mu \mathrm{m}$. Die Dicke der Strukturen ist $d=100 \mathrm{~nm}$.

Nach Mittelung über die Strukturgröße von $5 \times 5 \mu m^{2}$ lässt sich nun die mittlere Magnetisierung der einzelnen Quadrate berechnen, deren Verlauf in Abhängigkeit des äußeren Feldes in Abb. 7.4 dargestellt ist. Exemplarisch wurde dies an den in den Abbildungen 7.2 bzw. 7.3 umrahmten Strukturen durchgeführt. Um die zu den Magnetisierungszuständen zugehörigen Domänenkonfigurationen detaillierter analysieren zu können, wurde der gleiche Ummagnetisierungszyklus an den CoPt-Strukturen mittels magnetischer Kraftmikroskopie untersucht. Die Konfigurationen, die sich am Beispiel des Quadrates mit mittlerem 
Nukleationsfeld (rot markiert) aus den MOIF- und MFM-Messungen (also $H_{z}$-Kontrast bzw. $\left.\partial H_{z} / \partial z\right)$ ergeben, sind unter dem Graphen zu ausgewählten Gegenfeldern zusätzlich dargestellt.

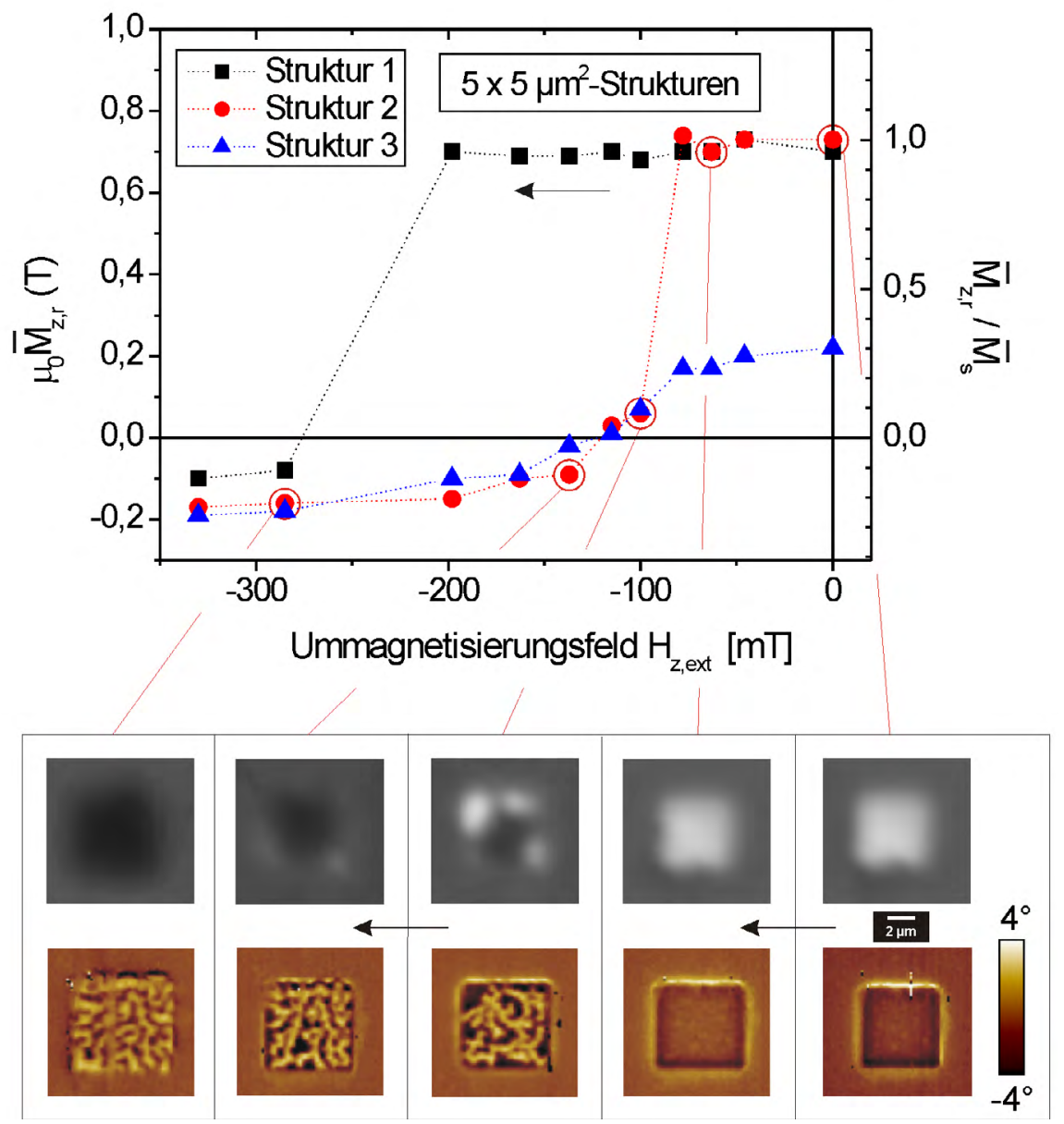

Abbildung 7.4: (Linke Ordinate) Über die Strukturgröße gemittelte remanente Magnetisierung der in den Abb. 7.2 und 7.3 markierten Quadrate als Funktion des angelegten Ummagnetisierungsfeldes. Aufgrund der reduzierten Ortsauflösung nach dem Fourierfiltern (vgl. Kap. 6) sind die gemittelten Magnetisierungswerte unterschätzt. (Rechte Ordinate) Gemittelte Magnetisierung normiert auf die gemittelte Sättigungsmagnetisierung, die durch MFM-Aufnahmen bestätigt wird, als Funktion von $H_{z, \text { ext }}$. Der Pfeil deutet die Erhöhung des externen Feldes in negativer Richtung an. Unter den Ummagnetisierungsverläufen sind zu ausgewählten Datenpunkten des rot markierten Quadrates aus Abb. 7.3 die jeweiligen Domänenkonfigurationen dargestellt, wie sie mittels MOIF (obere Reihe) und magnetischer Kraftmikroskopie (untere Reihe) abgebildet werden. Die Pfeile zeigen ebenfalls die Richtung der Ummagnetisierungsserie an.

Das MFM-Bild des Ausgangszustandes bestätigt in diesem Fall noch einmal den eindomänigen, homogen senkrecht aufmagnetisierten Zustand der Struktur, die damit die Sättigungsmagnetisierung aufweist. Eine Reduktion der mittleren Magnetisierung bei den MOIF-Messungen durch nicht aufösbare Gegendomänen kann damit ausgeschlossen werden. Auf Basis dessen ist in Abb. 7.4 eine auf den gemittelten Sättigungswert normierte Auftragung an der rechten Ordinate zusätzlich dargestellt. 
Im weiteren Verlauf der Kurve zeigt die rot markierte Struktur einen sprunghaften Abfall der Magnetisierung bei einem negativen externen Feld zwischen $-80 \mathrm{mT} \geq H_{z, \text { ext }} \geq-100$ mT. Der Effekt einer Nukleation von streifenartigen, entgegengesetzt aufmagnetisierten Domänen ist in dem MFM-Bild klar erkennbar. Aus dem Vergleich mit dem magnetooptischen Bild lässt sich gut der Grad an Mittelung der MOIF-Methode erschließen. Ausgedehntere Bereiche ohne Gegendomänen spiegeln sich im MO-Bild klar wider (Vergleich weißer Bereich in der linken oberen Ecke), während solche mit sehr feiner Domänenstruktur in einem sehr gleichmäßigen dunklen Kontrast erscheinen.

Eine weitere Erhöhung des angelegten Gegenfeldes führt nach dem sprunghaften Abfall nur noch zu einer schwachen Abnahme der mittleren Magnetisierung und die Ummagnetisierung verläuft kontinuierlich . $\chi$ ist unterhalb des Koerzitivfeldes, das in diesem Fall $\mu_{0} H_{c} \approx 120 \mathrm{mT}$ beträgt, also deutlich reduziert.

Die beiden zusätzlich markierten Quadrate zeigen in ihrem Ummagnetisierungsverhalten einen anfangs veränderten Verlauf. Die Struktur mit großem Schaltfeld (schwarz) bleibt entsprechend lang in seinem eindomänigen Zustand und besitzt damit ein deutlich erhöhtes Nukleations- und Koerzitivfeld. Das zweite Quadrat (blau umrandet) hingegen besitzt bereits im Ausgangszustand eine Multidomänenkonfiguration. Hier reicht schon die magnetostatische Selbstenergie der Struktur aus, um eine Nukleation von Gegendomänen zu erreichen, so dass bereits auf der positiven Feldachse eine Ummagnetisierung einsetzt. Das Koerzitivfeld dieser Struktur ergibt sich mit $\mu_{0} H_{c}=-120 \mathrm{mT}$ zu einem Wert, den auch schon die rot markierte Struktur aufweist, obwohl die remanenten Ausgangszustände verschieden sind. Die übrigen Strukturen besitzen Werte für $\mu_{0} H_{c}$ zwischen -180 mT und $<-330 \mathrm{mT}$. Hierin zeigt sich zum einen, wie lokal abhängig die einzelnen Ummagnetisierungsprozesse von Strukturen dieser Größe verlaufen, aber im Gegensatz dazu auch wie universell die Größe $\mu_{0} H_{c}$ für einen Großteil der Einzelstrukturen ist. Man kann bei Analyse nur eines Quadrats also nicht von einem repräsentativen Ummagnetisierungsverhalten für alle anderen Strukturen sprechen.

Als Vergleich zu den $5 \mu m$-Quadraten, um den Einfluss der Strukturgröße zu untersuchen, soll im Folgenden das Ummagnetisierungsverhalten der CoPt-Quadrate mit einer größeren Kantenlänge $(10 \mu m)$ und im Gegensatz dazu mit einer deutlich kleineren $(1 \mu m)$ beschrieben werden.

Abbildung 7.5 zeigt dazu den Ummagnetisierungsprozess für die $10 \times 10 \mu m^{2}$ und $1 \times 1 \mu m^{2}$ Strukturen. Prinzipiell lässt sich feststellen, dass der Ausgangszustand der großen Quadrate nach Aufmagnetisieren durch mehr Elemente im Multidomänenzustand geprägt ist. Die quantitative Auswertung in Abbildung 7.6 zeigt wieder, dass bis zu dem für alle Strukturen nahezu gleichen Koerzitivfeld im Bereich $\mu_{0} H_{c} \approx-115$ bis $-140 \mathrm{mT}$, die Verläufe je nach Ausgangskonfiguration nur anfangs verschieden sind. Es tritt dabei wieder sowohl der sprunghafte Abfall in der Magnetisierung (z.B. schwarzes Quadrat) mit einer anschließenden reduzierten Suszeptibilität auf, als auch von vorneherein kontinuierliche Magnetisierungsänderungen (z.B. blaues Quadrat). Der Bereich für die auftretenden Nukleationsund Koerzitivfelder sämtlicher Strukturen erweist sich als deutlich enger, verglichen mit den $5 \mu m$-Strukturen, die ein breiteres Intervall gezeigt hatten.

Die $1 \times 1 \mu m^{2}$-Strukturen wurden aus bereits genannten Gründen quantitativ nicht ausgewertet. Rein qualitativ zeigt sich hier, dass das Schalten der einzelnen Elemente bei noch 
a.)

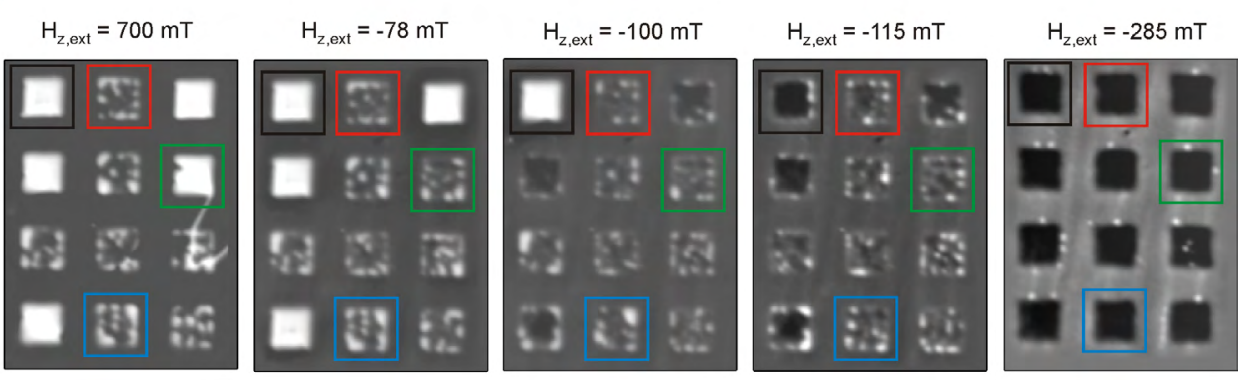

b.)

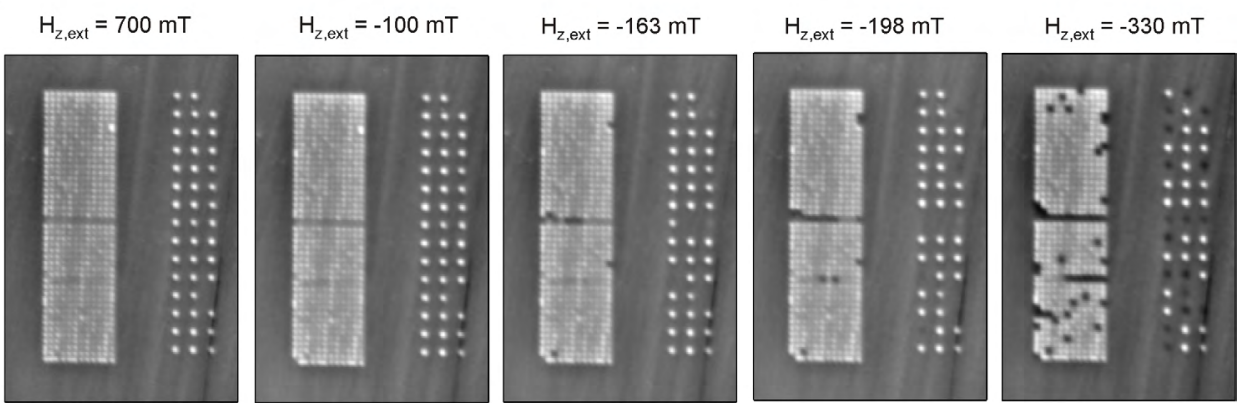

Abbildung 7.5: Ummagnetisierungsverhalten von (a) $10 \times 10 \mu m^{2}$ und (b) $1 \times 1 \mu m^{2}$ CoPtStrukturen bei verschiedenen, über den Bildern angegebenen, Gegenfeldern. Die kleinen Strukturen in (b) haben einen Abstand von $1 \mu m$ (linkes Feld) und $5 \mu m$ (rechtes Feld). In der Mitte des großen Feldes ist ein Strukturierungsfehler zu erkennen.

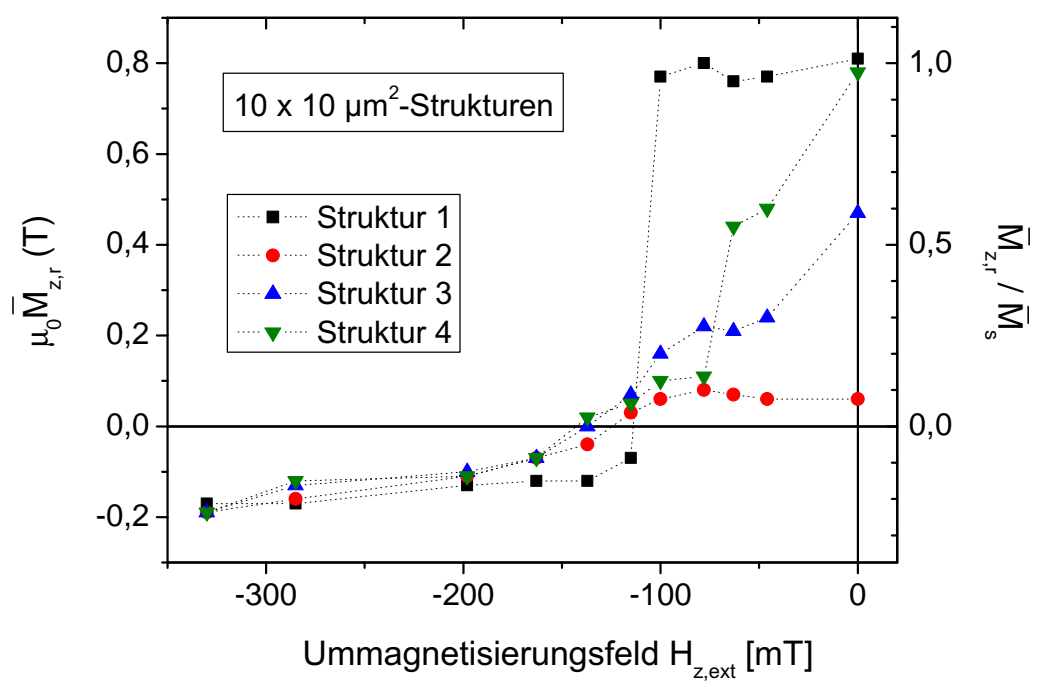

Abbildung 7.6: Über die Strukturgröße gemittelte remanente und normierte Magnetisierung der in Abb. 7.5 umrandeten $10 \times 10 \mu \mathrm{m}^{2}$ Quadrate als Funktion des angelegten Ummagnetisierungsfeldes.

größeren Gegenfeldern stattfindet als bei den größeren Strukturen. Desweiteren erkennt man, dass die geschalteten Quadrate nicht statistisch über das gesamte Strukturenfeld homogen verteilt liegen, sondern in bestimmten Bereichen gleich mehrere ummagnetisierte Quadrate zu finden sind, während andere ausgedehnte Bereiche noch kein einziges um- 
magnetisiertes Quadrat beinhalten. Hier erweist sich der große Überblick als besonders wichtig, da man lokal sehr unterschiedliche Verhältnisse bekommen würde, könnte man nur Teile der Anordnung analysieren.

Abbildung 7.7(a) zeigt, dass die $1 \times 1 \mu \mathrm{m}^{2}$-Strukturen ebenfalls über Nukleation und Wachstum von Gegendomänen ummagnetisiert werden. Es zeichnen sich typische Domänengrößen im Bereich von 50 bis $200 \mathrm{~nm}$ ab. Der prinzipielle Einfluss der Strukturgrösse auf das Ummagnetisierungsverhalten der Quadrate geht aus Abb. 7.7(b) hervor. Anhand des prozentualen Anteils aller in den Multidomänenzustand übergegangenen Quadrate als Funktion der Kantenlänge und des externen Feldes zeigt sich deutlich, dass kleinere Elemente größere Nukleationsfelder benötigen.

a.)

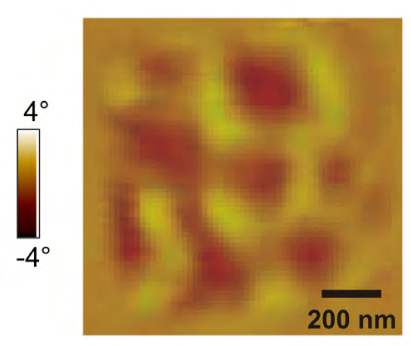

b.)

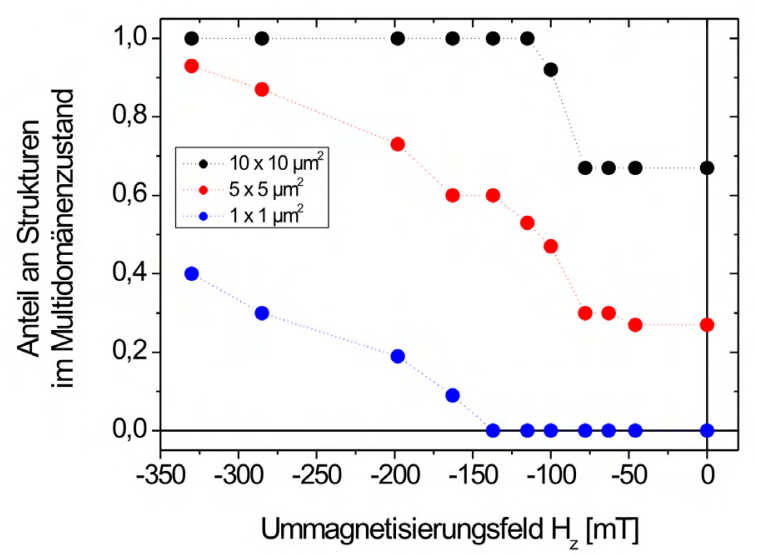

Abbildung 7.7: (a) MFM-Bild eines CoPt-Quadrates der Kantenlänge $1 \mu \mathrm{m}$ in einer Multidomänenkonfiguration nach Anlegen eines Gegenfeldes (Stärke von $H_{z, e x t}$ nicht bekannt). (b) Darstellung des auf die Gesamtanzahl der Strukturen mit fester Größe bezogenen Anteils der in den Multidomänenzustand geschalteten Quadrate in Abhängigkeit des externen Feldes.

Zuletzt soll der Einfluss der Form der Strukturen auf das Ummagnetisierungsverhalten dargestellt werden. Abbildung 7.8(a) stellt die remanenten Streufeldverteilungen von Stegen unterschiedlicher Dimensionen nach Anlegen entsprechender Gegenfelder dar. Hierbei zeigt sich nun zwischen den Strukturen mit $5 \mu \mathrm{m}$ als kurzer Seite gegenüber den Stegen mit $1 \mu \mathrm{m}$ ein völlig gegensätzliches Bild. Die großen Stege besitzen als Ausgangszustand nach Aufmagnetisieren einen Multidomänenzustand, während sich die kleineren Stege im Gegensatz dazu alle in einem eindomänigen befinden. An den beiden mit 1 und 2 markierten Stegen erkennt man, dass hier sowohl Konfigurationen auftreten können, in denen ein ganzer Bereich des Volumens noch eindomänig ist (Struktur 1 linker Rand) oder die Gegendomänen gleichmäßig über die gesamte Struktur auftreten (Steg 2). In den Magnetisierungskurven dieser beiden Strukturen in Abb. 7.8(b) zeigt sich dies in den unterschiedlichen Anfangsverläufen der Kurven. Der Sprung in der Magnetisierung bei Struktur 1 zwischen $\mu_{0} H_{z}=-78 \mathrm{mT}$ und $\mu_{0} H_{z}=-100 \mathrm{mT}$ ist in (a) dabei als ein Ummagnetisieren des großen eindomänigen Bereiches an der linken Seite zu erkennen, der aus der Sättigung in einen Zustand mit negativer mittlerer Magnetisierung springt, während das restliche Volumen seine Konfiguration nur schwach ändert. Die großen Stege besitzen mit $\mu_{0} H_{c} \approx 120 \mathrm{mT}$ ein Koerzitivfeld, das dem der $10 \mu \mathrm{m}$-Quadrate gleicht. 
a.)
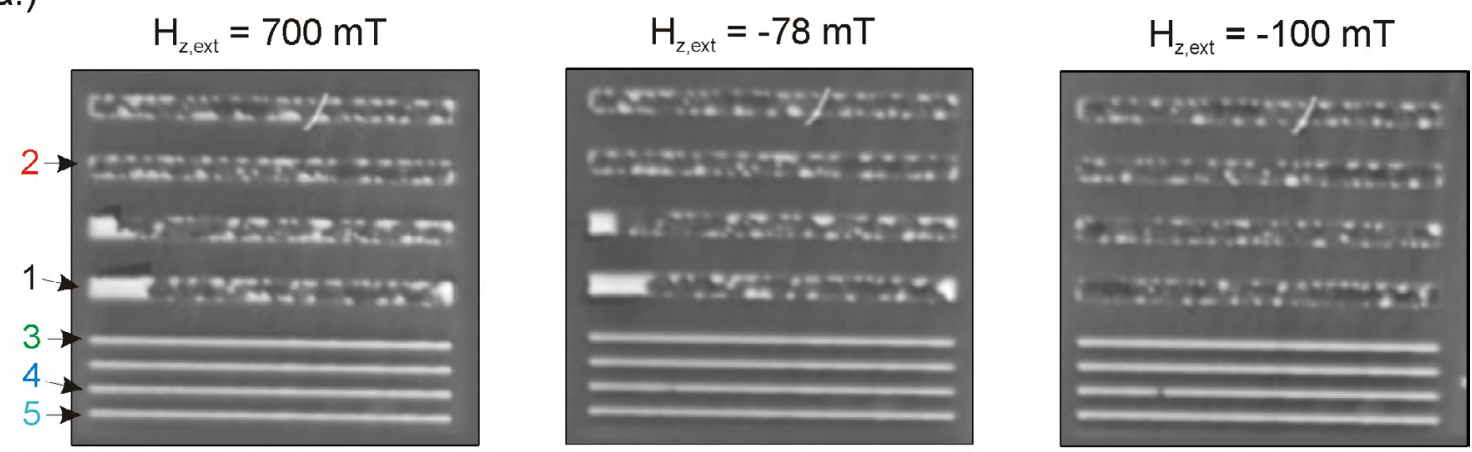

$H_{z, e x t}=-163 m T$

$$
H_{z, e x t}=-198 m T
$$
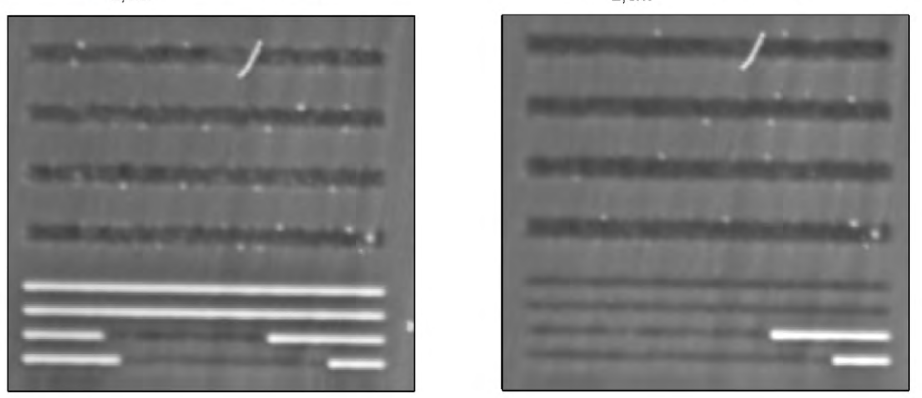

$$
\mathrm{H}_{\mathrm{zext}}=-330 \mathrm{mT}
$$

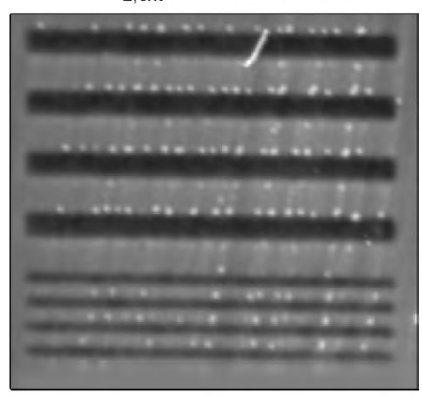

b.)

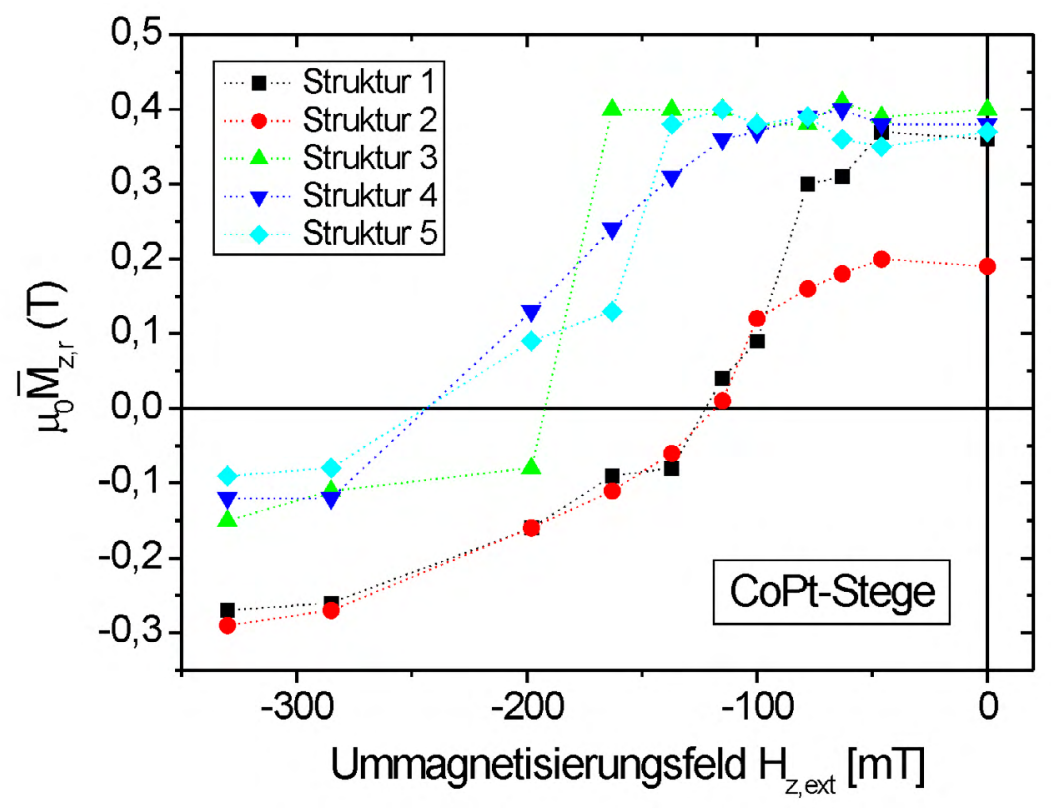

Abbildung 7.8: (a) Ummagnetisierungsverhalten von $5 \times 80 \mu \mathrm{m}^{2}$ und $1 \times 80 \mu \mathrm{m}^{2}$ CoPt-Stegen bei steigenden Gegenfeldern. Die weißen Punkte im letzten Bild sind kleine Domänen im Indikator und haben nichts mit der Magnetisierungsstruktur der Stege zu tun. (b) Über die Strukturgröße gemittelte remanente und normierte Magnetisierung der in (a) markierten Stege als Funktion des angelegten Feldes.

Die kleineren Stege zeigen ein vergleichbares Verhalten zu den $1 \mu m$ Quadraten. Im Ausgangszustand sind sie alle eindomänig und halten diesen Zustand auch sehr lange aufrecht. Dies erscheint ungewöhnlich, da auch die Stege durch ihre lange Seite von $80 \mu m$ eine recht 
große Fläche haben. An den Strukturen in den Bildern zu $\mu_{0} H_{z}=-163 \mathrm{mT}$ und $-198 \mathrm{mT}$ erkennt man gut, dass der Ummagnetisierungsprozess wieder über sehr große Bereiche des Volumens und innerhalb eines sehr schmalen Feldintervalls stattfindet. Interessant ist z.B., dass während die beiden oberen kleinen Stege bei einem externen Feld $\mu_{0} H_{z}=-198$ mT komplett in den mehrdomänigen Zustand übergegangen sind, man bei den beiden unteren erkennt, dass lediglich die linken grossen Domänen ummagnetisiert wurden. Die Domänenwände sind dagegen nicht in den rechten Teil hineingelaufen. Hier scheint eine größere Barriere für die Wandbewegung zu existieren, als das im linken Bereich der Fall war. Dies lässt hier schon sehr gut erkennen, wie die Defektverteilung in den Strukturen und dem daraus entstehenden pinning der Domänenwände das explizite Ummagnetisierungsverhalten beeinflußt.

\subsection{Diskussion des Ummagnetisierungsverhaltens der CoPt-Strukturen}

Die im vorherigen Abschnitt dargestellten magneto-optischen und magnetischen Kraftmikroskopieaufnahmen bestätigen bei allen Strukturgrößen den Ummagnetisierungsprozess über die Nukleation von Gegendomänen mit anschließendem Wachstum durch Domänenwandverschiebung. Der Nukleationsprozess zeigt sich dabei stark abhängig von der Kantenlänge der Quadrate (vgl. Abb. 7.7(b)). Die Breite der Verteilung der charakteristischen Kenngrößen Nukleationsfeld $H_{n}$ und Koerzitivfeld $H_{c}$ ist für die verschiedenen Strukturfelder deutlich unterschiedlich und lokal geprägt. Die Werte, die sich aus den dargestellten remanenten Magnetisierungskurven ergeben, erweisen sich gegenüber dem Film, aus dem die Strukturen präpariert wurden, als leicht erhöht. Dessen Magnetisierungskurve ist in Abb. 7.9 noch einmal dargestellt.

Es ist erkennbar, dass vor allem die Koerzitivfeldstärke des Filmes kleiner ist als diejenigen der strukturierten Elemente (vgl. Abb. 7.4 und 7.6). Dies kann verschiedene Ursachen haben, die im Folgenden genauer analysiert werden sollen.

Eine mögliche Ursache liegt in der Reduktion der magnetostatischen Energie bei sinkender Strukturgröße. Bei einer gleichgehaltenen Schichtdicke $(d=100 \mathrm{~nm})$ ändert sich mit der Strukturgröße das Aspektverhältnis und damit das Verhältnis der Entmagnetisierungsfaktoren $N_{z}$ zu $N_{x}$ bzw. $N_{y}$. Der Formanisotropiebeitrag $E_{d e m, z}=0,5 N_{z} M_{s}^{2}$ sinkt dementsprechend mit kleiner werdender Strukturgröße [87]. Vernachlässigt man bei der Betrachtung zunächst die hohen Anisotropiefelder, so führt dieser Effekt rein qualitativ bereits dazu, dass die Koerzitivfelder mit abnehmender Kantenlänge zunehmen. Um dies für die obigen Strukturgrößen quantitativ besser abschätzen zu können, wurden die Entmagnetisierungsfaktoren in Abhängigkeit des Verhältnisses $w / d$ bestimmt (Dicke der Strukturen $d$ und Kantenlänge $w$ ). Unter der Annahme die Strukturen würden durch die Form eines oblaten Sphäroiden angenähert, was für Stukturen mit großem $w / d$ eine sinnvolle Näherung darstellt [87, 130], kann man anhand der Gleichungen 7.5 und 7.6 


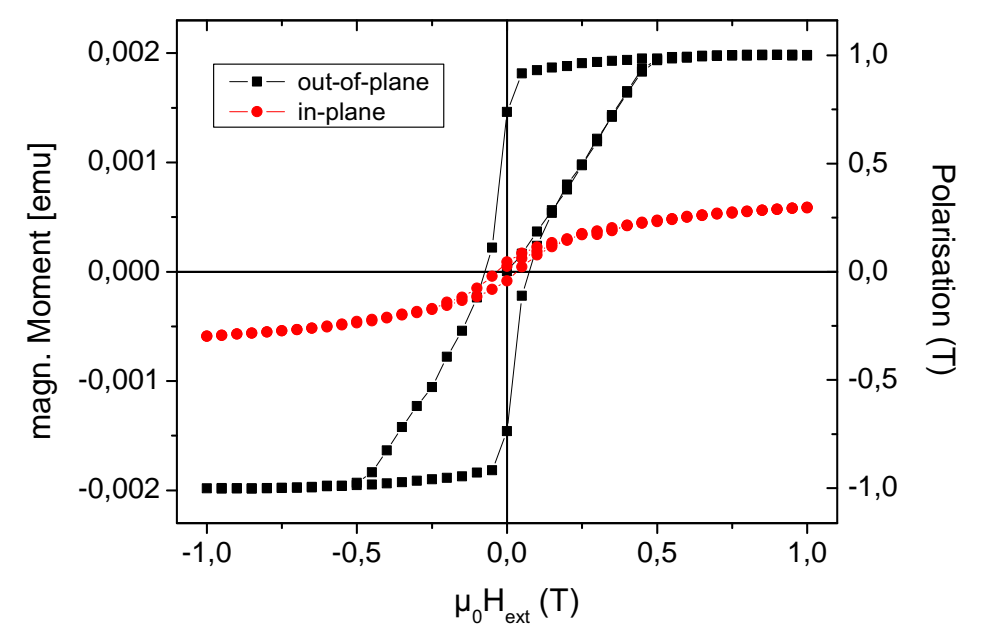

Abbildung 7.9: SQUID-Messung an dem ausgedehnten CoPt-Film, aus dem die untersuchten Strukturen präpariert wurden. Das Koerzitivfeld ergibt sich zu $\mu_{0} H_{c}=75 \mathrm{mT}$ und das Anisotropiefeld zu $\mu_{0} H_{a}=6,7 \mathrm{~T}$. Aus $H_{a}$ folgt mit der Sättigungsmagnetisierung des CoPt von $\mu_{0} M_{s}=1 \mathrm{~T}$ eine uniaxiale Anisotropiekonstante von $K_{u}=2,7 \mathrm{erg} / \mathrm{cm}^{3}$. Die Remanenz liegt bei $M_{r}=0,74 M_{s}$ und die Schicht hatte im $\theta-2 \theta$ Röntgendiffraktogramm einen Ordnungsgrad $S=0,80$ gezeigt.

$$
\begin{aligned}
N_{x}=N_{y} & =\frac{1}{2\left(m^{2}-1\right)} \cdot\left[\frac{m^{2}}{\sqrt{m^{2}-1}} \arcsin \left(\frac{\sqrt{m^{2}-1}}{m}\right)-1\right] \\
N_{z} & =1-2 \cdot N_{x}
\end{aligned}
$$

mit $m=w / d$, den Entmagnetisierungsfaktor $N_{z}$ berechnen [131]. Für den einfachen Grenzfall eines "unendlich" dünnen, in der Ebene ausgedehnten Films ergibt sich $N_{z}=1$. In Tabelle 7.1 sind die resultierenden Werte zusammengefasst.

\begin{tabular}{|c||c|c|c|}
\hline$w[\mu m]$ & 10 & 5 & 1 \\
\hline$N_{z}$ & 0,9845 & 0,9694 & 0,8608 \\
\hline
\end{tabular}

Tabelle 7.1: Entmagnetisierungsfaktor $N_{z}$ als Funktion der Kantenlänge der quadratischen Strukturen bei fester Dicke $d=100 \mathrm{~nm}$.

Der Entmagnetisierungsfaktor der Strukturen mit den größeren Kantenlängen entspricht praktisch dem einer dünnen Schicht. Die Veränderung des Koerzitivfeldes, die sich aus einer reinen Reduktion der magnetostatischen Selbstenergie bei kleiner werdenden Strukturgrössen ergibt, kann unter Verwendung der verschiedenen $N_{z}$ aus dem Entmagnetisierungsfeld berechnet werden. Mit der Differenz $\Delta H_{\text {dem }}=-\Delta N_{z}(m) \cdot M_{s}$ erwartet man eine Erhöhung von $\mu_{0} H_{c}$ zwischen dem kontinuierlichen CoPt-Film und z.B. den $10 \mu \mathrm{m}$ Strukturen von $15 \mathrm{mT}$. Für Quadrate mit einer Kantenlänge von $5 \mu \mathrm{m}$ resultiert gegenüber den mit $10 \mu \mathrm{m}$ eine weitere Erhöhung des Koerzitivfeldes um etwa $16 \mathrm{mT}$.

Ein Vergleich mit den Ummagnetisierungskurven der Strukturen zeigt, dass die experimentell gefundenen Werte für das Koerzitivfeld über diesen berechneten liegen. Da sich 
die Strukturen lokal sehr unterschiedlich verhalten, ist es an dieser Stelle sinnvoll, den Vergleich über alle Strukturen einer Größe zu betrachten. Dazu kann man die gemittelten, integralen Werte der Magnetisierung berechnen. Abbildung 7.10 gibt das Ergebnis dieser Mittelung in Form der Ummagnetisierungskurve für den Mittelwert der remanenten Magnetisierung aller Strukturen einer Grösse als Funktion des extern angelegten magnetischen Feldes wieder.

Es zeigt sich, dass die Mittelung für die Remanenz der großen Quadrate einen sogar etwas kleineren Wert als den des Filmes ergibt. Dies kann eventuell auf Bereiche mit reduzierter Ordnung zurückzuführen sein, die beim Ionenstrahlätzen der Strukturen an den Rändern entstanden sind. Das Koerzitivfeld erweist sich mit $\mu_{0} H_{c}=125 \mathrm{mT}$ um $50 \mathrm{mT}$ größer als das des Filmes. Die kleineren Strukturen besitzen im Mittel eine höhere Remanenz und zeigen mit $\mu_{0} H_{c} \approx 260 \mathrm{mT}$ bereits eine deutlich erhöhte Koerzitivität. Es ist damit offensichtlich, dass aus Gründen einer reinen magnetostatischen Energieabsenkung die Zunahme des Koerzitivfeldes und auch des Nukleationsfeldes nicht erklärt werden kann.

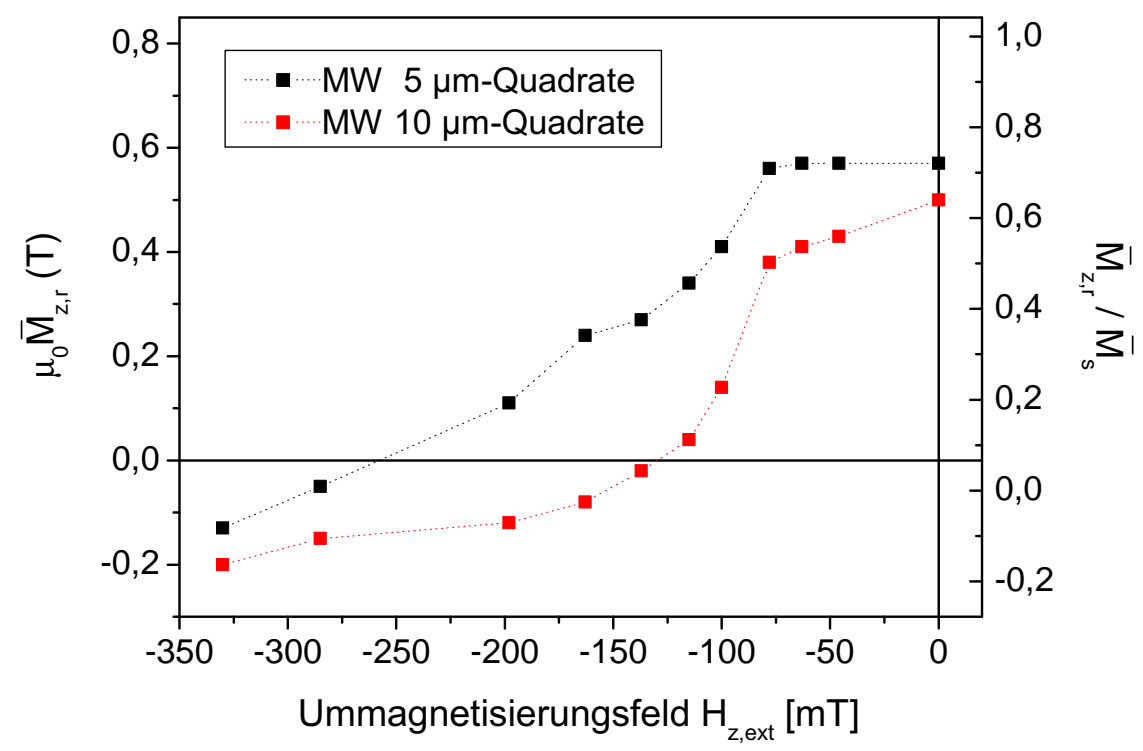

Abbildung 7.10: Remanente, über alle Quadrate gleicher Größe gemittelte, Magnetisierung als Funktion des externen Feldes.

Für das Ummagnetisierungsverhalten scheinen somit im Wesentlichen nur die mikrostrukturellen Defekte als Bereiche mit reduzierter Ordnung verantwortlich zu sein, die als Nukleationszentren wirken. Die experimentellen Ergebnisse geben aber schon klare Hinweise wie diese Defekte beschaffen sein müssen.

i) Abbildung 7.11 zeigt MFM-Aufnahmen der Frühstadien des Ummagnetisierungsprozesses an den $5 \mu \mathrm{m}$-Strukturen. Man erkennt klar, dass die Nukleation von Gegendomänen nur an sehr wenigen Stellen stattfindet. Vor allem tritt sie nicht bevorzugt am Rand auf, sondern im Volumen. Damit sind strukturierungsbedingte Defekte, z.B. durch eine Beschädigung der Ränder bei dem Abtrag mittels Ionenbestrahlung, auszuschliessen. Desweiteren zeigt sich, dass nach der Bildung von Gegendomänen sehr kleine Felder ausreichen, um ein signifikantes Domänenwachstum durch Wandverschiebungen hervorzurufen. 
ii) Individuelle Unterschiede im Ummagnetisierungsverhalten treten besonders bei Strukturgrößen von $\leq 5 \mu \mathrm{m}$ auf, während bei den großen Anordnungen die einzelnen Quadrate ein sehr ähnliches zeigen (vgl. Abb. 7.2 und 7.5). Dies impliziert konsistent zu i), dass der mittlere Abstand starker Keimbildungszentren, die zu einem sehr geringen Nukleationsfeld führen, im $\mu m$-Bereich liegt und nur wenige dieser Zentren das Ummagnetisierungsverhalten bestimmen.

iii) Es reicht nicht aus, den Nukleationszentren einen mittleren Abstand zuzuordnen, da sich das Ummagnetisierungsverhalten als formabhängig erweist. Man findet deutliche Unterschiede für die annähernd flächengleichen Quadrate und Stege (vgl. Abb. 7.8).

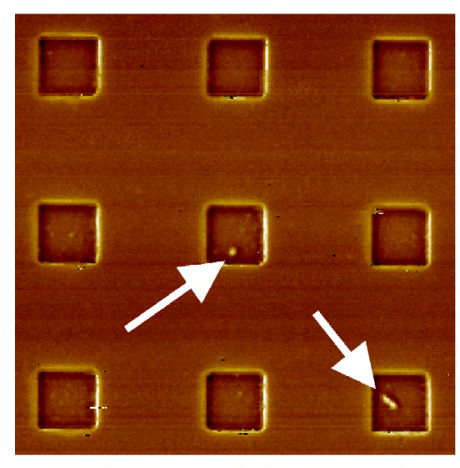

$\mu_{0} \mathrm{H}_{z, \mathrm{ext}}=700 \mathrm{mT}$

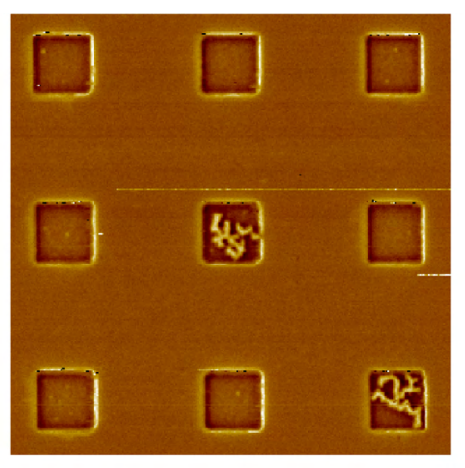

$\mu_{0} \mathrm{H}_{z, \mathrm{ext}}=-45 \mathrm{mT}$

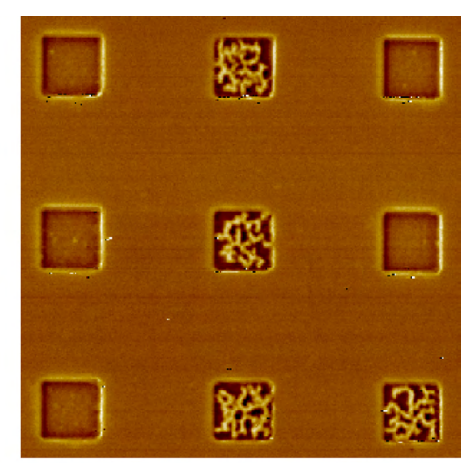

$\mu_{0} \mathrm{H}_{z, \mathrm{ext}}=-63 \mathrm{mT}$

Abbildung 7.11: MFM-Bilder zum Anfangsstadium der Nukleation von Gegendomänen (durch weiße Pfeile markiert) in den $5 \mu \mathrm{m}$ großen Quadraten und dessen Wachstum in kleinen Feldern.

Die begrenzte Auflösung der magneto-optischen Messmethode lässt prinzipiell keine eindeutige Abgrenzung zwischen Nukleation und Wachstum durch Wandverschiebungen zu. Es gibt damit zwei mögliche Szenarien, die die dargestellten Ergebnisse erklären würden. Das erste basiert auf einem größen- und formabhängigen Wachstumsprozess, das zweite auf einer größen- und formabhängigen Nukleationswahrscheinlichkeit.

Im ersten Fall ist es denkbar, dass sich sehr kleine Gegendomänen, die mit der MOIFMethode nicht aufgelöst werden können, bei allen Strukturen in nahezu gleichen externen Feldern bilden, aber das größen- und formabhängige Entmagnetisierungsfeld im Innern der Strukturen zu einem unterschiedlichen Wachstum führt. $H_{d e m}$ ist der Magnetisierung entgegengerichtet und besitzt damit, zumindest im Zentrum einer Struktur, einem dem Betrag von $M$ gleichen, aber negativen Wert. Zu den Rändern der Probe fällt $H_{\text {dem }}$ stark ab, wobei die Ausdehnung dieser im Entmagnetisierungsfeld reduzierten Randbereiche in das Volumen hinein von der Größe der Struktur abhängig ist [43]. Die treibende Kraft der Domänenwandverschiebung liegt in einer Differenz der magnetostatischen Energie zwischen einer Domäne mit der ursprünglichen Magnetisierungsrichtung und einer Gegendomäne. Je größer das Entmagnetisierungsfeld in der Umgebung der Gegendomäne, umso größer ist der magnetostatische Energiegewinn. Die Domänenwandbewegung in Richtung der Ränder ist somit stärker gehemmt, da hier der Energiegewinn aufgrund des reduzierten Entmagnetisierungsfeldes nicht mehr so groß ist [132]. Wird die Grundfläche einer Struktur also kleiner, so ist $H_{d e m}$ lediglich im Zentrum der Struktur noch maximal. In Simulationen [133] konnte allerdings gezeigt werden, dass der Unterschied zwischen $10 \mu \mathrm{m}$ und $5 \mu \mathrm{m}$-Strukturen sehr gering ist und das Entmagnetisierungsfeld nur in einem sehr 
schmalen Bereich am Rand abfällt. Dies erklärt also weder die deutlichen Unterschiede im Nukleationsverhalten, die sowohl zwischen den $10 \mu m$ - und $5 \mu m$-Strukturen als auch zwischen den Elementen gleicher Größe herrschen. In Strukturen mit $1 \mu m$ Kantenlänge ist $H_{\text {dem }}$ noch in etwa $60 \%$ des Volumens voll ausgeprägt, so dass auch hier eine veränderte Wandbeweglichkeit in Kombination mit dem begrenzten Auflösungsvermögen der MOIF keine tatsächliche Erklärung für die obigen Ergebnisse liefert.

Die Diskussion der Nukleationswahrscheinlichkeit in Abhängigkeit der Form und Größe der Strukturen erfordert zunächst eine Erläuterung, welche Defekte prinzipiell als Keimbildungzentren im Sinne einer heterogenen Keimbildung in Frage kommen. Zum einen treten in den magnetischen Elementen die gleichen präparationsbedingten Defekte, wie sie auch in epitaktisch abgeschiedenen Filmen vorkommen auf. Abhängig von den Depositionstemperaturen beträgt die typische Inselgröße in solchen Filmen bis $>100 \mathrm{~nm}$, es können in diesen aber auch granulare Substrukturen mit reduzierter Grösse (5 - $15 \mathrm{~nm})$ vorhanden sein [104, 134], so dass nach der Koaleszenz der Inseln ein sehr dichtes Korngrenzennetzwerk besteht. Weiterhin findet man Defekte wie Stapelfehler, Antiphasen- und Zwillingsgrenzen sowie Versetzungen und Anti-Site-Defekte, deren mittleren Abstände klein sind gegen die hier betrachteten Strukturgrößen. Somit müsste in allen Strukturen eine hinreichend hohe Anzahl von Nukleationszentren vorliegen, so dass ein größenunabhängiges homogenes Ummagnetisieren der Anordnungen zu erwarten und die Bildung vieler Domänen in den Frühstadien des Prozesses zu erkennen wäre. Dies ist nach Abb. 7.11 und den dargestellten Ergebnissen aber auszuschließen. Ein weiteres wichtiges Argument dagegen stellt auch die sehr kleine Domänenwandbreite des CoPt dar, die etwa $\delta_{D W} \approx 5 \mathrm{~nm}$ beträgt. Man kann davon ausgehen, dass solche Fehler an die geordneten Körner austauschgekoppelt sind und wesentlich höhere Nukleationsfelder benötigen.

Als starke Keimbildungszentren kommen damit vor allem ungeordnete, weichmagnetische Körner in Frage, die grundsätzlich auftreten, da in den CoPt-Filmen (FePt) keine Ordnungsgrade von $S=1$ erreicht werden. Die Ordnungseinstellung ist nach [135] ein Phasenübergang 1. Ordnung, so dass in einem Film mit $S<1$ ein Zweiphasengemisch aus geordneten und ungeordneten Körnern existiert [136]. Skomski et. al. berechnen das theoretische Nukleationsfeld eines kugelförmigen, weichmagnetischen Einschlusses in einer hartmagnetischen Matrix als Funktion des Anisotropiefeldes und der Domänenwandbreite der Matrix, sowie des Radius des Einschlusses [137]. Es zeigt sich, dass das Nukleationsfeld eines solchen Einschlusses stark herabgesetzt ist. Für CoPt mit einer Domänenwandbreite von $\delta \approx 5 \mathrm{~nm}$ und dem experimentell gefundenen Anisotropiefeld von $\mu_{0} H_{a} \approx 6,5$ T (siehe Abb. 7.9) erhält man bei Körngrößen im Bereich von 20 - $40 \mathrm{~nm}$ Werte für das Nukleationsfeld von $\mu_{0} H_{n}=100$ - $400 \mathrm{mT}$, mit abnehmender Stärke für die größeren Durchmesser. Die Größenordnung der Werte liegt auch in dieser Arbeit vor. Dies impliziert allerdings, dass die ungeordnete Phase in einer Korngrößenverteilung vorliegt, so dass nur ein vergleichsweise kleiner Volumenanteil der Körner die relevante Größe hat, damit der entsprechende Abstand groß ist. Dies scheint prinzipiell möglich, wenn man als Verteilungsfunktion der Korngrößen des Filmes, aus dem die Strukturen präpariert wurden, eine Gaußverteilung annimmt, mit einer Varianz im Bereich weniger Nanometer [138, 139]. Aus einer entsprechenden Abschätzung mittels einer normierten Gaußverteilung unter Annahme eines mittleren Korndurchmessers von $30 \mathrm{~nm}$ und einer Varianz von 5-6 nm ergibt sich eine untere Grenze für den mittleren Abstand der $40 \mathrm{~nm}$ Körner von 
3-6 $\mu \mathrm{m}$.

Unter diesen Voraussetzungen lassen sich auch die Unterschiede zwischen den kleinen Stegen mit $1 \mu \mathrm{m}$ als kurzer Seite und den Quadraten trotz fast identischer Flächengleichheit erklären. Für die Quadrate steigt die Wahrscheinlichkeit ein starkes Nukleationszentrum innerhalb der Struktur zu finden mit Zunahme der Kantenlänge quadratisch an. Für die Stege hingegen, bei denen in einer Dimension die Kantenlänge deutlich kleiner ist als der mittlere Abstand der Keimbildungszentren, nimmt mit steigender Kantenlänge der langen Seite und gleichbleibender kurzer Seite die entsprechende Wahrscheinlichkeit nur linear zu. Detailliertere Analysen erfordern sicherlich die Untersuchung des Gefüges mittels Transmissionselektronenmikroskopie um die räumliche Verteilung, bzw. besonders die Korngrößenverteilung quantitativ besser erfassen zu können.

Abschließend lässt sich feststellen, dass gerade das Ummagnetisierungsverhalten der kleinen Stege gezeigt hat, dass bei der Charakterisierung großer Anordnungen auf lokaler Ebene die Aussagen auf mesoskopischer Skala nicht repräsentativ sind und zu einem detaillierten Verständnis sowohl die großen Messausschnitte der MOIF als auch hochaufgelöste Messungen mit dem MFM wichtig sind. 


\section{Ansatz zur Kalibrierung eines magnetischen Kraftmikroskops mittels quantitativer MOIF-Messungen}

Nachdem im vorhergehenden Kapitel besonders der große Messausschnitt der MOIFMethode zur quantitativen Untersuchung des Ummagnetisierungsverhaltens ausgedehnter Strukturanordnungen genutzt wurde, steht in diesem Kapitel der Nutzen der quantitativen Magnetisierungsrekonstruktion im Vordergrund. Dabei geht es um die Möglichkeit der Kalibrierung eines magnetischen Kraftmikroskops unter Verwendung der quantitativ bestimmten Magnetisierungsverteilungen von FePt-Kalibrierstandards durch die MOIFMethode. Die magnetische Kraftmikroskopie wurde bereits in Kapitel 7 als hochauflösende, streufeldsensitive Methode zu einem qualitativen Vergleich mit magneto-optischen Aufnahmen herangezogen. Die Schwäche der Methode ist, wie schon in Kapitel 3.5 kurz erläutert, dass sie aufgrund der komplexen Wechselwirkung der magnetischen Spitze mit dem zu untersuchenden Medium nur unter größerem Aufwand quantitative Informationen liefern kann.

Die quantitative Anpassung der beiden genannten Methoden basiert auf dem Transferfunktionenschema, welches, ähnlich wie bei der Magnetooptik, auch auf die magnetische Kraftmikroskopie angewendet werden kann [37, 38].

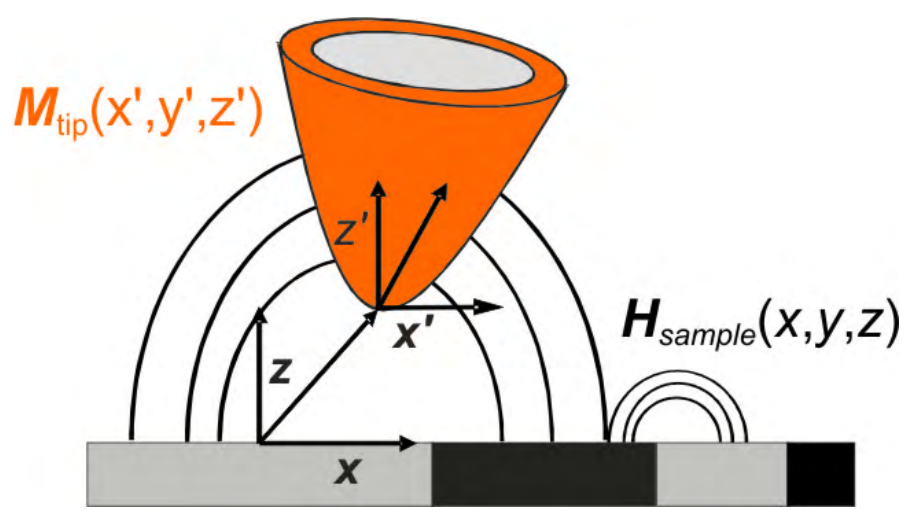

Abbildung 8.1: Schematische Darstellung des zugrundeliegenden Koordinatensystems, das die im weiteren verwendete Nomenklatur festlegt.

Die Kraft, die auf eine magnetische Spitze mit der Magnetisierungsverteilung $\vec{M}_{\text {Tip }}\left(\vec{r}^{\prime}\right)$ im Streufeld einer Probe wirkt (vgl. Abb. 8.1), wird in Analogie zur Theorie in Kapitel 2 im zweidimensionalen Fourierraum durch das Integral 


$$
\vec{F}(\vec{k}, z)=-\int_{-\infty}^{\infty} \vec{H}_{\text {Probe }}\left(\vec{k}, z+z^{\prime}\right) \vec{\nabla} \cdot \vec{M}_{\text {Tip }}^{*}\left(\vec{k}, z^{\prime}\right) d z^{\prime}
$$

bestimmt (* für komplex konjugiert). Bei einer räumlich begrenzten Probe muss die Lösung der Laplacegleichung (im Außenraum) für $z \longrightarrow \infty$ gegen Null gehen, so dass mit $\left.\vec{H}_{\text {Probe }}\left(\vec{k}, z+z^{\prime}\right)=\vec{H}_{\text {Probe }}(\vec{k}, z) \cdot \exp \left(-k z^{\prime}\right)\right)$ für die Gleichung 8.1

$$
\vec{F}(\vec{k}, z)=-\vec{H}_{\text {Probe }}(\vec{k}, z) \int_{-\infty}^{\infty} e^{-k z^{\prime}} \vec{\nabla} \cdot \vec{M}_{\text {Tip }}^{*}\left(\vec{k}, z^{\prime}\right) d z^{\prime}=-\vec{H}_{\text {Probe }}(\vec{k}, z) \cdot \sigma_{\text {Tip }}(\vec{k})
$$

folgt. Die sogenannte force transfer function $(F T F), \sigma_{\text {Tip }}(\vec{k})$, beschreibt eine effektive magnetische Ladungsdichte der Spitze, projeziert auf den Tip-Apex und spiegelt im weiteren die magnetischen Eigenschaften und Mikrostruktur der MFM Spitze wieder. Sie ist nur abhängig von $\vec{k}$ und nicht von der Messhöhe. Multipliziert man im Fourierraum die FTF mit der Feldtransferfunktion $\alpha(k)$ (vgl. Kap. 2), ohne zunächst eine Einschränkung auf die $H_{z}$-Komponente des Streufeldes der Probe zu machen, so erhält man die sogenannte tip transfer function, die somit die zugrundeliegende Magnetisierung der Probe mit der Kraft auf die MFM-Spitze korreliert. Weiterführende Details zu den jeweiligen Transferfunktionen und zu den mathematischen Formalismen sind in [37, 38, 60] aufgeführt. Im weiteren wichtig ist die Abhängigkeit der FTF von der räumlichen Frequenz.

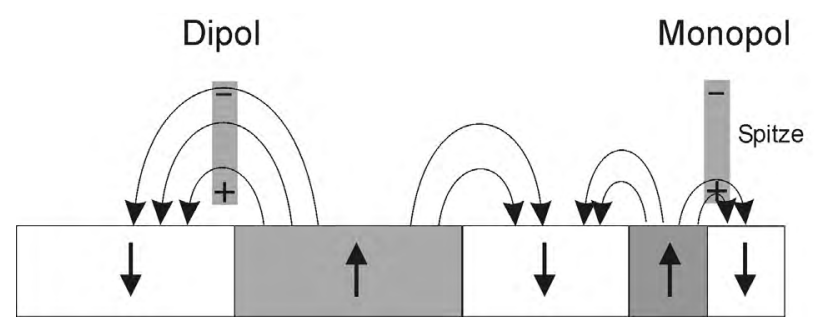

Abbildung 8.2: Schematische Darstellung der Wechselwirkung einer stabförmig angenäherten Spitze mit dem Probenstreufeld. Je nach Reichweite der Streufeldverteilung der Probe ergibt sich eine unterschiedliche Darstellung der Spitze als effektiver Monopol oder Dipol.

Abbildung 8.2 verdeutlicht schematisch die Problematik der $k$-Abhängigkeit unter Verwendung der in Kapitel 3 beschriebenen Modelle (Monopol-, Dipolnäherung) für die Wechselwirkung der Spitze mit dem Probenstreufeld. Bei Annahme einer stabförmigen, ausgedehnten Spitze ist die Kraft auf die Spitze proportional zum Streufeld, wenn dieses auf einer Länge deutlich kleiner als die der Spitze abfällt. In diesem Fall kann die Monopolnäherung angewendet werden, die z.B. für kleine Wellenlängen (grossen Wellenvektoren) sinnvoll ist. Im umgekehrten Fall großer Wellenlängen und schwach abfallender Streufelder spührt die gesamte Spitze eine Kraft, die proportional zu $d H_{z} / d z$ ist und die Dipolbeschreibung geeigneter macht. Tritt bei der MFM-Messung ein kontinuierliches Wellenvektorspektrum auf, so überlagern sich die Effekte abhängig von der Länge der Spitze und machen eine Beschreibung mittels Monopol- bzw. Dipolnäherung wertlos für 
quantitative Aussagen. Desweiteren ist zu bedenken, dass die Spitze eine nicht idealisierte Form wie in dem obigen Beispiel besitzt und die Messungen so geometrieabhängig werden.

Bei der Abbildung im dynamischen Messmodus wird die Spitze in eine erzwungene Schwingung versetzt und über die Probe gerastert. Das resultierende Messignal ist die Phasenverschiebung $\Delta \psi$ oder die Frequenzverschiebung $\Delta f$ gegenüber der Anregung. Beide sind proportional zu $\frac{d}{d z} F_{z}$. Wird als Beispiel die Frequenzverschiebung gewählt, so folgt für das Messsignal

$$
\Delta f=c \cdot \sigma_{\text {Tip }}(\vec{k}) \frac{d}{d z} \vec{H}_{\text {Probe }}(\vec{k}, z) \equiv \operatorname{ICF}(\vec{k}) \cdot \frac{d}{d z} \vec{H}_{\text {Probe }}(\vec{k}, z)
$$

mit einer Konstante $c$, die im wesentlichen die relevanten mechanischen Eigenschaften des Cantilevers beschreibt. Gleichung 8.3 stellt damit die Definitionsgleichung der MFMKalibrierfunktion (instrument calibration function $(I C F)$ ) dar, die es erlaubt die Messdaten in Feldstärkewerte umzurechnen, wenn sie einmal für eine Spitze bekannt ist. Um die $I C F$ zu bestimmen, benötigt man eine Probe mit einer bekannten Streufeldverteilung, an der die Kalibrierung mittels Gl. 8.3 vorgenommen werden kann.

Im Folgenden soll gezeigt werden, dass Gleichung 8.3 einen wesentlich umfassenderen Zugang zur Beschreibung der Wechselwirkung der Spitzenmagnetisierung mit dem Probenstreufeld liefert, als die in Kapitel 3 vorgestellte Beschreibung von $\vec{M}_{\text {Tip }}$ als effektives Monopol- oder Dipolmoment. Dies kann durch den Vergleich mit magneto-optischen Messungen im Folgenden untermauert werden. Für die weitere Betrachtung sollen die Konsequenzen der sogenannten Point-Probe Approximation noch einmal kurz dargelegt werden. Im Rahmen dieser Beschreibung kann die Phasenverschiebung der Spitze $\Delta \phi$, die proportional zu dem Gradienten der Kraft auf die Spitze ist, dargestellt werden als [81]

$$
\Delta \phi=k \cdot \frac{\partial F_{z}}{\partial z}=k \cdot q \frac{\partial H_{z}}{\partial z}+m_{z} \frac{\partial^{2} H_{z}}{\partial z^{2}}
$$

Der Monopolterm führt also zu einer Abhängigkeit des MFM-Signals von der 1. Ableitung des Streufeldes, der Dipolterm zu einer von der 2. Ableitung.

Als Kalibrierstandards für einen Vergleich von MFM und MOIF können nun die gleichen FePt-Strukturen verwendet werden, die auch bereits als Teststrukturen der Etablierung der Magnetisierungsrekonstruktions für die MOIF dienten. Die quantitative Bestimmung der Magnetisierung dieser Proben mittels magneto-optischer Indikatorfilm-Methode ermöglicht im weiteren die Berechnung der Gradienten der $H_{z}$-Komponente in der Messhöhe der MFM-Untersuchungen, also in der Höhe des Tip-Apex und läßt so einen quantitativen Vergleich zwischen den Messmethoden zu. Abbildung 8.3 zeigt die MFM-Messung an den FePt-Strukturen (a) im Vergleich zu den beiden magneto-optischen Bildern der $d H_{z} / d z$ und $d^{2} H_{z} / d z^{2}$-Komponente, (c) bzw. (b), die sich nach Berechnung aus der rekonstruierten Magnetisierungsverteilung in Abb. 6.6 aus Kap. 6.1 ergeben. Die beiden gerechneten MOIF-Bilder stellen damit die Fälle einer reinen Monopol- bzw. reinen Dipolspitze dar.

Neben der unterschiedlichen experimentellen Ortsaufösung der Bilder erkennt man rein qualitativ vor allem zwischen der berechneten 1. Ableitung des Streufeldes und dem MFMBild eine recht gute Übereinstimmung. Die Profile entlang der Linien in den Aufnahmen, 
a.)

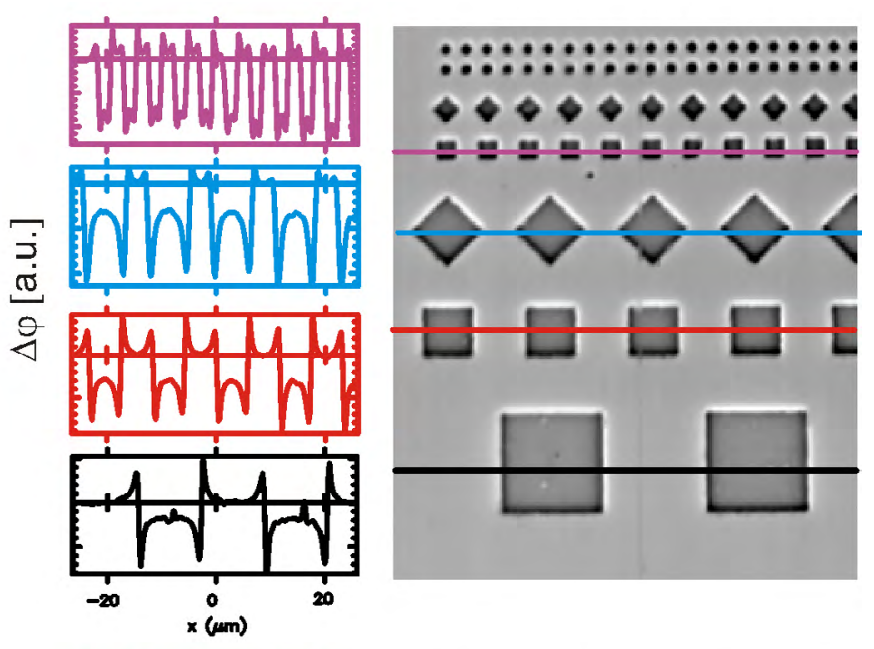

b.)

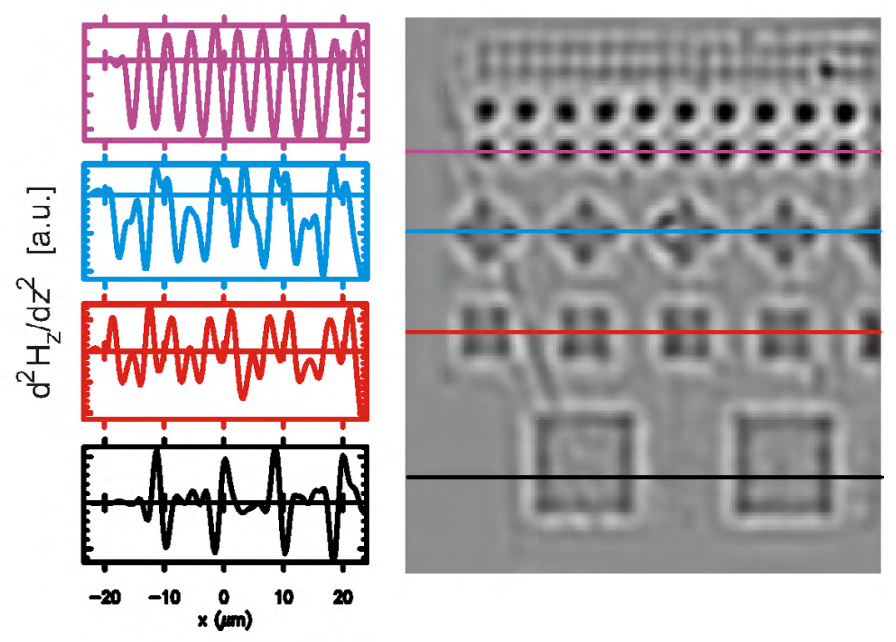

c.)

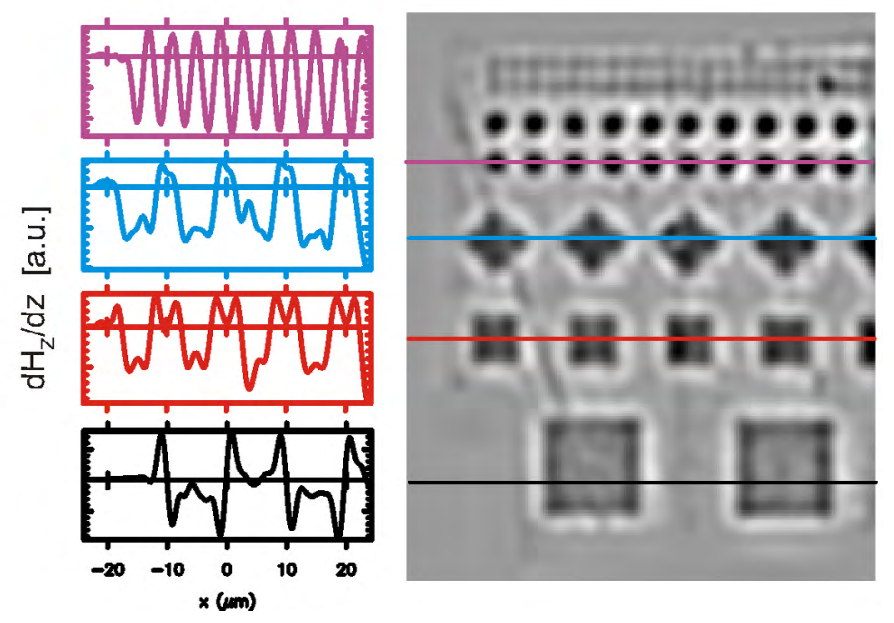

Abbildung 8.3: (a) MFM-Messung in einer Messhöhe von $100 \mathrm{~nm}$ an einem Strukturenfeld aus FePt-Quadraten verschiedener Größe. Die Profile geben das Messsignal entlang der Linien im Bild wieder. (b) Berechnung der $d^{2} H_{z} / d z^{2}$ - und (c) der $d H_{z} / d z$-Verteilung in $100 \mathrm{~nm}$ Höhe aus der rekonstruierten Magnetisierungverteilung nach MOIF-Messung (Abb. 6.6).

bestätigen dies. Gerade der Vergleich der Verläufe direkt über den $5 \mu m$ und den $10 \mu m$ Strukturen in (a) und (c) zeigen, dass die Werte nicht auf Null zurückgehen, sondern 
einen endlichen Wert behalten. Bei der 2. Ableitung in (b) ist dies nicht der Fall, dort sind nur an den Kanten der größeren Elemente die Werte ungleich Null. Die $2 \mu m$ Strukturen lassen einen sinnvollen Vergleich nicht mehr zu, da man aufgrund der begrenzten Auflösung in den magneto-optischen Bildern hier keinen Unterschied mehr zwischen den beiden Ableitungen erkennt.

Zur Bestimmung der ICF müssen die Bilder fouriertransformiert werden und man erhält eine 2-dim. diskrete Spektraldarstellung der Fourierkomponenten in Abhängigkeit der räumlichen Frequenzen $k_{x}$ und $k_{y}$. Da die z-Differentation der Streufeldkomponente im Frequenzraum nach Gleichung 8.2 eine Multiplikation mit $k$ darstellt, ist es für einen einfachen Vergleich sinnvoll, sowohl den Betrag der Koeffizienten zu berechnen als auch deren Abhängigkeit vom Betrag des Wellenvektors. Hierbei ist zu bedenken, dass dies nur Informationen über die Transformation der Stärke der Streufeldgradienten gibt, die fehlende Phaseninformation jedoch eine Rekonstruktion der Bilder nicht zulässt. Abb. 8.4 zeigt 1-dim. Fourierspektren zu den obigen drei Bildern. Dabei wurde bei der Berechnung der räumlichen Frequenz, $k=\sqrt{k_{x}^{2}+k_{y}^{2}}$, die y-Komponente im Wert gleich Null festgehalten $\left(k_{y}=0\right)$. Dem größten k-Wert entspricht die Pixelauflösung der Bilder, dem kleinsten die Länge des gesamten Bildausschnittes. In Abb. 8.4 ist aber nur der relevante Frequenzbereich dargestellt. Beide Ableitungen des Streufeldes wurden auf das Hauptmaximum des MFM-Signals bei $\lambda=10 \mu m$ skaliert, um den relativen Verlauf besser vergleichen zu können.

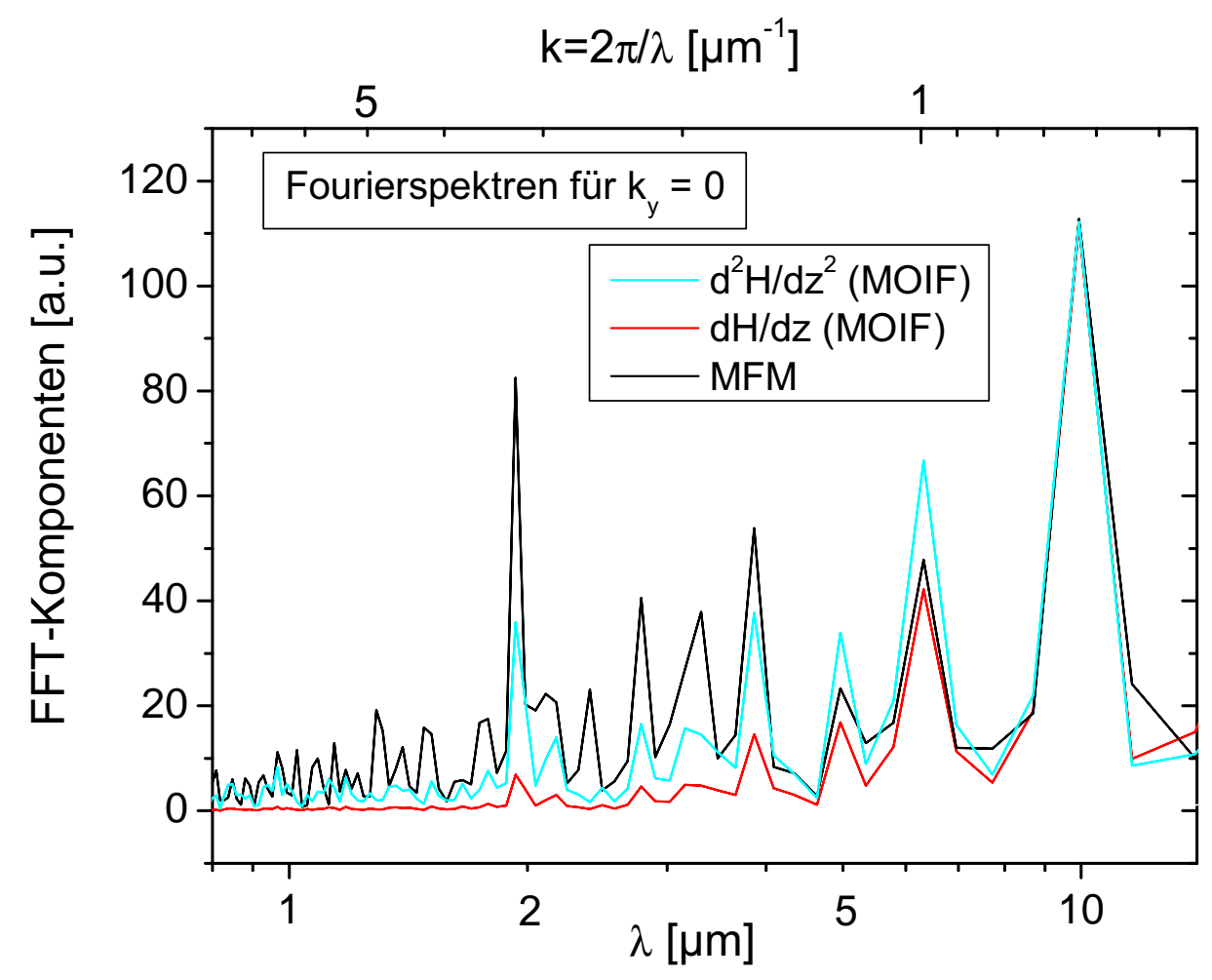

Abbildung 8.4: Fourierspektren der 3 Aufnahmen aus Abb. 8.3. Die Beträge der Fourierkomponenten sind als Funktion der räumlichen Frequenz für einen festen Wert $k_{y}=0$ dargestellt. Die 2. Ableitung des Streufeldes wurde der besseren Vergleichbarkeit auf das Maximum des MFMSignals normiert. Prinzipiell besitzt diese Größe natürlich mit $m^{-2}$ eine andere Einheit als der 1. Gradient. 
Zunächst erkennt man eine starke Korrelation aller Spektren durch die sehr charakteristischen Maxima genau bei den Wellenlängen, die den entsprechenden Hauptfourierkomponenten der Quadrate zuzuordnen sind, nämlich bei $\lambda=10,5$ und $2 \mu \mathrm{m}$. Darüberhinaus sind auch zwischen diesen Werten mehr oder weniger stark ausgeprägte Maxima zu sehen, die den Fourierkomponenten höherer Ordnung entsprechen. Entscheidend für den weiteren Vergleich ist der relative Abfall der Fourierkomponenten mit sinkender Wellenlänge, bzw. steigender Frequenz. Bis zu $\lambda=5 \mu m$ zeigt sich eine bessere Übereinstimmung zwischen dem MFM-Signal und der 1. Ableitung des Streufeldes. Der Gradient 2. Ordnung erweist sich in diesem Wertebereich noch als zu groß. Für Werte $\lambda<5 \mu m$ ändert sich dieses Verhalten aber und der Verlauf des $d^{2} H_{z} / d z^{2}$-Spektrums spiegelt sich sehr viel ausgeprägter im MFM-Spektrum wieder, vor allem zu kleinen Wellenlängen $(<2 \mu m)$. Die experimentelle Ortsauflösung in den MOIF-Bildern beträgt 1, $7 \mu \mathrm{m}$. Unterhalb dieser Wellenlänge sollte aufgrund der Abschneidewellenzahl des Hanning-Fensters kein Signal mehr erkennbar sein. Allerdings mussten die unterschiedlichen Pixelanzahlen des MFM-Bildes und der beiden MOIF-Bilder für einen sinnvollen Vergleich aneinander angepasst werden, so dass in den MOIF-Spektren auch noch Fourierkomponenten zu kleineren Wellenlängen erkennbar sind. In dem Bereich unterhalb von 1,7 $\mu \mathrm{m}$ ist in den Spektren der Ableitungen aber davon auszugehen, dass es sich eher um Rauschen handelt. Zusammenfassend zeigt sich jedoch in Abb. 8.4 deutlich, dass weder die Dipolnäherung in Form der 2. Ableitung von $H_{z}$ noch die Monopolnäherung über den einfachen Gradienten ein zufriedenstellendes Ergebnis liefert. Beide Näherungen können also nur ein sehr eingeschränktes Bild der Wechselwirkung der Spitze mit dem Streufeld und damit eine sehr ungenügende Beschreibung für eine Quantifizierung der Signale geben. Prinzipiell zeigt sich auch, dass für ganz bestimmte Probendimensionen jeweils eine der Näherungen besser passt. Für die Quantifizierung eines MFM-Bildes, in dem aber sehr viele k-Vektoren enthalten sind, stellt die Ermittlung der $I C F$ jedoch die einzig sinnvolle Herangehensweise dar.

Die Bestimmung der $I C F$ ist prinzipiell möglich, indem man das MFM-Spektrum durch das der 1. Ableitung dividiert. Es zeigt sich aber, dass der Quotient vor allem zu kleinen Wellenlängen hin stark von Rauschen dominiert wird und eine einfache Division keinen Sinn macht. Im Bereich der großen Wellenlängen wäre prinzipiell eine Bestimmung der $I C F$ durchführbar, jedoch sind in den Messbildern aufgrund der drei verschiedenen Strukturgrößen nur sehr begrenzt Fourierkomponenten in dem zugänglichen Wellenlängenbereich vorhanden. Generell erweist sich die begrenzte Auflösung der MOIF-Methode als Hindernis einer Anpassung über einen größeren Bereich, so dass insgesamt der Überlapp an gemessenen räumlichen Frequenzen zu gering ist. Dennoch kann die MOIF-Methode durch ihre lokale Bestimmung der Magnetisierung einen Beitrag zur Verbesserung der Kalibrierung gegenüber den in der Literatur verwendeten Verfahren leisten. Die $I C F$, so wie sie z.B. von Hug et al. oder auch van Schendel et al. berechnet wird, basiert auf theoretischen Streufeldsimulationen, die als Eingangsinformation einen, mittels SQUID-Messung bestimmten, integralen Magnetisierungwert von ausgedehnten Filmen verwenden. Die Kalibriertransferfunktion wird dann in einem iterativen Prozess solange angepasst, bis die Fourierspektren der berechneten Streufeldverteilung und das experimentelle MFM-Signal eine minimale Abweichung voneinander besitzen. Hierbei ist zu bedenken, dass die Magnetisierung lokale Inhomogenitäten aufweisen kann, wenn in den abgebildeten Materialsystemen lokale Defektstrukturen auftreten. Beispielsweise kann in geordneten Systemen, wie sie in den vorherigen Kapiteln gezeigt wurden, diese nicht vollständig gegeben 
sein oder eine abweichende Stöchiometrie zu einer veränderten lokalen Magnetisierung führen. Insofern stellt die lokale Bestimmung von $\vec{M}$ mittels MOIF eine vertrauenswürdigere Startinformation für die Berechnung der $I C F$ aus der theoretischen Simulation von Streufeldverteilungen dar. Zusätzlich ist zu bedenken, dass die Magneto-Optik auf einer etwas größeren Längenskala ein Bild der zugrundeliegenden Domänenstruktur liefern kann. Prinzipiell können die Fourierkomponenten der großen Wellenlängen auch zur späteren Verifizierung herangezogen werden. Für die explizite Bestimmung der ICF müssen jedoch weitergehende numerische Verfahren basierend auf Gleichung 8.3 entwickelt werden. 
8. Ansatz zur Kalibrierung eines magnetischen Kraftmikroskops mittels quantitativer 


\section{Zusammenfassung}

Im Rahmen der vorliegenden Arbeit wurde der Fragestellung einer voll quantitativen lokalen Rekonstruktion von Magnetisierungsverteilungen aus Messungen der Normalkomponente des Streufeldes kleiner magnetischer Strukturen sowohl aus theoretischer Sicht als auch von der Warte einer technischen Realisierbarkeit mit der verwendeten MOIFMethode nachgegangen. Die Entwicklung eines theoretischen Formalismus auf Basis von Transferfunktionen, der alle intrinsischen Messbedingungen der magneto-optischen Methode berücksichtigt, ermöglichte dabei einen umfassenden Zugang zur Lösung des zugrundeliegenden inversen magnetostatischen Problems. Grundlage für die experimentelle Umsetzung der Rekonstruktion war die Optimierung der Messmethode und die Präparation von geeigneten Teststrukturen.

Die experimentelle Basis bildet ein Polarisationsmikroskop, dass bezüglich des Auflösungsvermögens und der Sensitivität im Zuge der Arbeit deutlich verbessert werden konnte und so die quantitative Streufelddetektion von Strukturen mit Dimensionen bis unterhalb von $1 \mu \mathrm{m}$ gewährleistet. Das Implementieren einer optischen Lock-In-Technik, dessen Herzstück ein programmierbarer Faraday-Rotator darstellt, sowie die Verwendung sehr dünner optisch aktiver Sensoren, resultieren dabei mit $\sigma \approx 800 \mathrm{~nm}$ in einer experimentellen Auflösung nahe der theoretischen Grenze $(\sigma \approx 670 \mathrm{~nm})$. Als essentiell für die Rekonstruktion erweist sich die Erhöhung des Signal-Rausch-Verhältnisses durch Mittelung über sehr viele Messbilder bei gleichzeitig sehr langen Einzelbildbelichtungszeiten, da das experimentelle Rauschen durch die inverse Problematik einen erheblichen Einfluss auf die Güte des Ergebnisses hat.

Ausgehend von dem modellhaften Charakter für die Untersuchung der Beziehung von Streufeld und zugrundeliegender Magnetisierung, wurden mittels Elektronenstrahllithographie Strukturen mit wohldefinierter Form und Größe präpariert, die ein definiertes Streufeld generieren. Dazu wurden verschiedene Materialsysteme verwendet, die die Realisierung zunächst einfacher, wohldefinierter Magnetisierungsverteilungen gewährleisten. Als Modellsystem für rein senkrechte Magnetisierungszustände wurden $L 1_{0}$-geordnete, hartmagnetische $\mathrm{Fe}_{0.5} \mathrm{Pt}_{0.5^{-}}$und $\mathrm{Co}_{0.5} \mathrm{Pt}_{0.5}$-Filme mit uniaxialer magnetokristalliner Anisotropie mittels Magnetron-Kosputtern hergestellt. Die Verwendung epitaxiefähiger Substrate wie $\mathrm{MgO}(001)$ und gleichzeitig hohe Depositionstemperaturen im Bereich um 670 ${ }^{\circ} \mathrm{C}$ resultieren dabei in FePt-Filmen mit sowohl hohen Ordnungsgraden von $S \approx 0,85$ als auch hohen Texturgüten bei einer vollständigen Ausrichtung der kristallographischen c-Achse entlang der Filmnormalen. Daraus ergeben sich wiederum sehr gute magnetische Eigenschaften mit einer ausgeprägten senkrechten Vorzugsachse der Magnetisierung und sehr hohe Remanenzen bei nicht zu geringen Schichtdicken, so dass die FePt-Elemente die Grundlage der Rekonstruktion für den Grenzfall rein senkrechter Magnetisierungsverteilungen bilden. 
Die CoPt-Filme zeigten dagegen nach der Herstellung ohne Pufferschichten ein bimodales Wachstum mit a-Achsen und c-Achsen orientierten Körnern und erwiesen sich als wenig geeignet für Teststrukturen, da die Mikrostruktur zu einer wenig definierten Magnetisierungskonfiguration führt. Bei Verwendung von Pt-Pufferschichten konnte ein c-Achsen orientiertes Wachstum induziert werden, allerdings waren der Ordnungsgrad und die Remanenz der Filme etwas niedriger als im FePt.

Für den zweiten Grenzfall rein in der Ebene ausgerichteter Magnetisierungszustände erwies sich das $\mathrm{PrCO}_{5}$-System aufgrund der sehr hartmagnetischen Eigenschaften ebenfalls als gut geeignet für detaillierte Untersuchungen bezüglich der Umsetzung der Magnetisierungsrekonstruktion.

Um die Messergebnisse hinsichtlich der quantitativen Streufeldbestimmung aus theoretischer Sicht interpretieren zu können, wurden Simulationen der z-Komponente des Streufeldes der experimentell realisierten Strukturen durchgeführt. Entscheidend ist hierbei die Berücksichtigung der endlichen aktiven Sensorschichtdicke und die begrenzte experimentelle Ortsauflösung der optischen Methode. So konnte vor allem gezeigt werden, dass die Ortsauflösung der Streufeldmessung im wesentlichen durch die endliche Sensordicke limitiert ist, während sich die Ortsauflösung nach der Magnetisierungsrekonstruktion als sehr sensibel auf die Sensordicke sowie auch auf den Rauschpegel in den Messungen erweist. Prinzipiell ließ sich eine sehr gute Übereinstimmung von Theorie und Experiment für alle untersuchten Strukturgrößen erzielen. Die Aussagekraft der Simulationen sowie die Gültigkeit der Sensorkalibrierung konnten so untermauert werden.

Die explizite Umsetzung der Magnetisierungsbestimmung aus den Streufeldmessungen war Gegenstand des 6. Kapitels. Unter Einbeziehung der experimentellen Limitierungen war es möglich Transferfunktionen für die MOIF-Methode zu entwickeln, die die Rekonstruktion der Magnetisierung für Strukturen mit Kantenlängen bis ca. 3-4 $\mu m$ quantitativ korrekt erlaubt. Sowohl bei den senkrechten als auch den inplane Magnetisierungsverteilungen erweist sich als begrenzender Faktor der Magnetisierungsbestimmung kleiner Strukturgrößen, die zur Rauschunterdrückung notwendige Fourierfilterung der Streufeldverteilung. Das Abschneiden von in der Transferfunktion verstärkten hohen räumlichen Frequenzen durch Verwendung eines Hanning-Fensters führt zu einer Reduktion der Ortsauflösung, so dass Elemente mit Kantenlängen kleiner als der Ortsauflösung einen reduzierten Magnetisierungswert nach der Rekonstruktion besitzen. Mittels theoretischer Simulationen konnte gezeigt werden, dass durch eine Optimierung der experimentellen Messbedingungen die Grenze der Rekonstruierbarkeit bei Strukturgrößen von etwa $2 \mu m$ liegt.

Aufbauend auf dem optimierten Abbildungsverfahren und dem entwickelten Inversionsformalismus wurden in Kapitel 7 die Frühstadien des Ummagnetisierungsverhaltens größerer Anordnungen hartmagnetischer CoPt-Strukturen verschiedener Form und Größe untersucht. Es konnte mittels magneto-optischer und Kraftmikroskopieuntersuchungen aufgezeigt werden, dass unter Vernachlässigung einer magnetostatischen Wechselwirkung zwischen den Strukturen die Ummagnetisierung einzig durch die Nukleation und das Wachstum von Gegendomänen geprägt ist. Als starke Nukleationszentren kommen dabei nur größere, ungeordnete Körner in Frage, so dass das Ummagnetisierungsverhalten durch eine mit der Form und der Größe der Strukturen skalierende Nukleationswahrscheinlichkeit im Sinne einer heterogenen Keimbildung erklärt werden kann.

Gegenstand des letzten Kapitels 8 war die Fragestellung einer möglichen Kalibrierung 
eines magnetischen Kraftmikroskops mittels quantitativer MOIF-Messungen an FePtEichstrukturen. Hierbei wurde aufgezeigt, dass es prinzipiell möglich ist, die Kalibrierung über die magneto-optische Messmethode durchzuführen. Es zeigte sich bei dem Vergleich der Fourierspektren der berechneten Ableitungen des Streufeldes mit dem des MFMSignals, dass beide Gradienten in dem Bild enthalten sind. Eine einfache Darstellung der Spitzenmagnetisierung über einen Monopol bzw Dipol würde aber quantitativ nicht zu korrekten Werten führen. Die Berechung der ICF für das MFM ist durch die begrenzte Auflösung der magneto-optischen Messmethode auf große Wellenlängen bis zu ca. $2 \mu m$ limitiert. Dennoch liefert die MOIF-Methode durch die lokale Magnetisierungsbestimmung sehr gute Referenzwerte als Ausgangsinformation für eine theoretische Simulation der Streufeldgradienten, auf denen im Weiteren eine numerische Bestimmung der MFMKalibrierfunktion aufgebaut werden kann.

Zusammenfassend lässt sich feststellen, dass die im Rahmen dieser Arbeit optimierte MOIF-Methode als quantitative und lokal gut aufösende Messmethode eine erweiterte Charakterisierung magnetischer Strukturen und deren Ummagnetisierungsverhalten ermöglicht. Besonders betont sei dabei die Möglichkeit, sowohl Streufeld- als auch Magnetisierungsverteilungen quantitativ zu bestimmen. So konnte zu einem besseren Verständnis der physikalischen Mechanismen bei der Ummagnetisierung hartmagnetischer Strukturen beigetragen werden.

Zusätzlich zu den im Detail dargestellten physikalischen Fragestellungen, wie z.B. der Analyse großer magnetischer Strukturanordnungen, ermöglichte der realisierte Messaufbau und das Verständnis der spezifischen Abbildungscharakteristika auch weiterführende Zusammenarbeiten mit technischem Anwendungsinteresse auf die hier nur kurz eingegangen werden soll. So wurden in diesem Zusammenhang in Zusammenarbeit mit dem Institut für Mikrotechnologie (Leibniz Universität Hannover) sowohl quantitative Messungen des Streufeldes einer Mikrospule als auch Streufelduntersuchungen an Einzelbauteilen von Mikromotoren durchgeführt. Hierbei wurde die vielseitige Einsetzbarkeit der MOIF-Methode eindrucksvoll demonstriert. Desweiteren konnte die MOIF-Methode zur Charakterisierung kollektiver, magnetostatisch induzierter Effekte von CoPt-Strukturen in einer $\mathrm{Ni}_{80} \mathrm{Fe}_{20}$-Matrix als auch bei der Bestimmung des Einflusses von Kantenrauigkeiten auf die Streufeldverteilung und das Ummagnetisierungsverhalten von FePt-Strukturen sinnvoll eingesetzt werden [46, 43]. Die in der Einleitung gesteckten Ziele und Ansprüche an die MOIF als weiterführende Methode können also abschließend als vollständig erfüllt bezeichnet werden. 


\section{A. Mathematischer Anhang}

\section{Mathematische Formeln}

Die Wirksamkeit der Deltafunktion unter einem Integral ist

$$
\int_{V} f\left(\vec{r}^{\prime}\right) \delta\left(\vec{r}^{\prime}-\vec{r}\right) d V\left(\vec{r}^{\prime}\right)=f(\vec{r}) .
$$

Die Fouriertransformierte einer Funktion $f(\vec{r})$ berechnet sich nach

$$
F(\vec{k})=\mathcal{F}\{f(\vec{r})\}=\int_{-\infty}^{\infty} e^{-i \vec{k} \vec{r}} f(\vec{r}) d^{3} r .
$$

Für die inverse Fouriertransformation gilt dann

$$
f(\vec{r})=\frac{1}{(2 \pi)^{3}} \int_{-\infty}^{\infty} e^{i \vec{k} \vec{r}} F(\vec{k}) d^{3} k .
$$

Wichtige Rechenregel für fouriertransformierte Funktionen ist ihr Verhalten im k-Raum bei Differentiation im Originalbereich (im 1-D):

$$
\mathcal{F}\left\{\frac{\partial^{n} f(r)}{\partial r^{n}}\right\}=(i k)^{n} \mathcal{F}\{f(r)\}
$$

Eine weitere sehr nützliche Anwendung der Fouriertransformation besteht bei der Faltung zweier Funktionen im Ortsraum

$$
f_{1}(\vec{r}) * f_{2}(\vec{r})=\int_{-\infty}^{\infty} f_{1}(\vec{\tau}) f_{2}(\vec{r}-\vec{\tau}) d^{3} \tau .
$$

Für die Fouriertransformation dieser sogenannten zweiseitigen Faltung gilt

$$
\mathcal{F}\left\{f_{1}(\vec{r}) * f_{2}(\vec{r})\right\}=\mathcal{F}\left\{f_{1}(\vec{r})\right\} \cdot \mathcal{F}\left\{f_{2}(\vec{r})\right\} .
$$

Es gilt weiterhin

$$
\mathcal{F}\left\{\delta\left(\vec{r}-\vec{r}^{\prime}\right)\right\}=1
$$


und für die Rücktransformation entsprechend

$$
\begin{aligned}
\delta\left(\vec{r}-\vec{r}^{\prime}\right) & =\frac{1}{(2 \pi)^{3}} \int_{-\infty}^{\infty} \mathcal{F}\left\{\delta\left(\vec{r}-\vec{r}^{\prime}\right)\right\} e^{i \vec{k}\left(\vec{r}-\vec{r}^{\prime}\right)} d^{3} k \\
& =\frac{1}{(2 \pi)^{3}} \int_{-\infty}^{\infty} e^{i \vec{k}\left(\vec{r}-\vec{r}^{\prime}\right)} d^{3} k
\end{aligned}
$$

Die obigen Gleichungen kann man nun verwenden, um die Fouriertransformierte der Greenschen Funktion für den gesamten $R^{3}$, die für das inverse Problem wichtig ist, zu bestimmen. Man startet mit $G\left(\vec{r}, \vec{r}^{\prime}\right)=\frac{1}{4 \pi} \frac{1}{\left|\vec{r}-\vec{r}^{\prime}\right|}$

$$
\Delta_{r} G\left(\vec{r}, \vec{r}^{\prime}\right)=-\delta\left(\vec{r}-\vec{r}^{\prime}\right) .
$$

Zunächst soll der 3D-Fall berechnet werden und daraus dann die für diese Arbeit wichtige 2D-Transformierte. Die Realraumfunktion berechnet sich aus der rücktransformierten Fouriertransformierten gemäß

$$
\begin{aligned}
\frac{1}{4 \pi\left|\vec{r}-\vec{r}^{\prime}\right|}=G\left(\vec{r}, \vec{r}^{\prime}\right) & =\frac{1}{(2 \pi)^{3}} \int_{-\infty}^{\infty} \mathcal{F}\left\{\frac{1}{4 \pi\left|\vec{r}-\vec{r}^{\prime}\right|}\right\} e^{i \vec{k}\left(\vec{r}-\vec{r}^{\prime}\right)} d^{3} k \\
& =\frac{1}{(2 \pi)^{3}} \int_{-\infty}^{\infty} G(\vec{k}) e^{i \vec{k}\left(\vec{r}-\vec{r}^{\prime}\right)} d^{3} k
\end{aligned}
$$

Somit folgt

$$
\begin{aligned}
\Delta_{r}\left\{\frac{1}{4 \pi\left|\vec{r}-\vec{r}^{\prime}\right|}\right\}=\Delta_{r} G\left(\vec{r}, \vec{r}^{\prime}\right) & =\frac{1}{(2 \pi)^{3}} \int_{-\infty}^{\infty} \mathcal{F}\left\{\frac{1}{4 \pi\left|\vec{r}-\vec{r}^{\prime}\right|}\right\} \Delta_{r} e^{i \vec{k}\left(\vec{r}-\vec{r}^{\prime}\right)} d^{3} k \\
& =\frac{1}{(2 \pi)^{3}} \int_{-\infty}^{\infty} \mathcal{F}\left\{\frac{1}{4 \pi\left|\vec{r}-\vec{r}^{\prime}\right|}\right\}(i k)^{2} e^{i \vec{k}\left(\vec{r}-\vec{r}^{\prime}\right)} d^{3} k \\
& =\frac{-1}{(2 \pi)^{3}} \int_{-\infty}^{\infty} \underbrace{\mathcal{F}\left\{\frac{1}{4 \pi\left|\vec{r}-\vec{r}^{\prime}\right|}\right\} k^{2}}_{\stackrel{!}{=} 1(\text { damit Gl.(A.8) erfüllt) }} e^{i \vec{k}\left(\vec{r}-\vec{r}^{\prime}\right)} d^{3} k \\
& =-\delta\left(\vec{r}-\vec{r}^{\prime}\right) \stackrel{(\mathrm{A} .8)}{=} \frac{-1}{(2 \pi)^{3}} \int_{-\infty}^{i \vec{k}\left(\vec{r}-\vec{r}^{\prime}\right)} d^{3} k \\
\Rightarrow & \mathcal{F}\left\{\frac{1}{4 \pi\left|\vec{r}-\vec{r}^{\prime}\right|}\right\} \cdot k^{2}=1 \\
& \mathcal{F}\left\{\frac{1}{4 \pi\left|\vec{r}-\vec{r}^{\prime}\right|}\right\}=\frac{1}{k^{2}}
\end{aligned}
$$


Das mathematische Problem aus der $H_{z}(\vec{r})$-Messung auf die Magnetisierung der Probe zurückzuschließen soll im Weiteren für den 2D-Fall betrachtet werden, bei dem die z-Komponente im Realraum behandelt wird und nur die $x, y$-Koordinaten in den Fourierraum transformiert werden müssen. Die Fouriertransformation der Greenschen Funktion hat als Ausgangspunkt die allgemeine Form aus Gl.(A.11). Im weiteren sind die 2DKomponenten im Fourierraum wie folgt:

$$
\vec{k}=\left(k_{x}, k_{y}\right) ; \quad|\vec{k}|=\sqrt{k_{x}^{2}+k_{y}^{2}}
$$

Damit ist zunächst

$$
G\left(\vec{k}, k_{z}\right)=\frac{1}{k^{2}+k_{z}^{2}} .
$$

Die Rücktransformation der z-Komponente ergibt dann

$$
\begin{aligned}
G\left(\vec{k}, z-z^{\prime}\right) & =\frac{1}{2 \pi} \int_{-\infty}^{\infty} \frac{1}{k^{2}+k_{z}^{2}} e^{i k_{z}\left(z-z^{\prime}\right)} d k_{z} \\
& =\frac{1}{2 \pi} \int_{-\infty}^{\infty} \frac{1}{\left(i k+k_{z}\right)\left(-i k+k_{z}\right)} e^{i k_{z}\left(z-z^{\prime}\right)} d k_{z}
\end{aligned}
$$

Die Funktion hat also zwei Pole bei $k_{z}= \pm i k$. Bei der Integration müssen diese Pole mitberücksichtigt werden. Eine physikalisch sinnvolle Lösung des Integrals findet man mit Hilfe des Residuensatzes.

Man betrachtet zunächst den Fall $\left(z-z^{\prime}\right)>0$, d.h. Werte $z>z^{\prime}$, da bei der gewählten Messgeometrie der Indikator über der zu messenden Probe liegt und deswegen $z$ in die positive Richtung gesehen größer ist als $z^{\prime}$. Es kommt bei der Wahl des Integrationsweges wie oben erwähnt darauf an, dass der Hilfsweg für $k_{z} \rightarrow \infty$ gegen Null geht.

$$
\begin{aligned}
e^{i k_{z}\left(z-z^{\prime}\right)} & =e^{i\left(\Re\left(k_{z}\right)+i \Im\left(k_{z}\right)\right)\left(z-z^{\prime}\right)} \\
& =\underbrace{e^{i \Re\left(k_{z}\right)\left(z-z^{\prime}\right)}}_{\leq 1} \cdot e^{-\Im\left(k_{z}\right)\left(z-z^{\prime}\right)} \leq e^{-\Im\left(k_{z}\right)\left(z-z^{\prime}\right)}
\end{aligned}
$$

Für $\left(z-z^{\prime}\right)>0$ muss also der Imaginärteil von $k_{z}>0$ gewählt werden, damit der Integrand für $k_{z} \rightarrow \infty$ exponentiell gedämpft wird und damit der Hilfsweg gegen Null geht. Wie man später sieht, ist der obere Weg nicht nur mathematisch sinnvoll, sondern auch physikalisch, denn die Greensche Funktion muss für $\vec{r} \rightarrow \infty$, bzw. $z \rightarrow \infty$ auch gegen Null gehen, was lediglich bei dem oberen Integrationsweg der Fall ist.

Wählt man also den oberen Weg, so umschließt man den Pol bei $k_{z}=i k$. Aus dem Residuensatz folgt damit

$$
G\left(\vec{k}, z-z^{\prime}\right)=2 \pi i\left\{\frac{1}{2 \pi} \frac{1}{(i k+i k)} e^{i(i k)\left(z-z^{\prime}\right)}\right\}=\frac{e^{-k\left(z-z^{\prime}\right)}}{2 k} \quad z-z^{\prime}>0
$$

Für Werte $z<z^{\prime}$ folgt eine allgemeine Darstellung über die Betragsfunktion für beliebige $z-z^{\prime}$. Die 2D-Fouriertransformierte der Punktquelle ist damit

$$
G\left(\vec{k}, z-z^{\prime}\right)=\frac{e^{-k\left|z-z^{\prime}\right|}}{2 k} \text {. }
$$




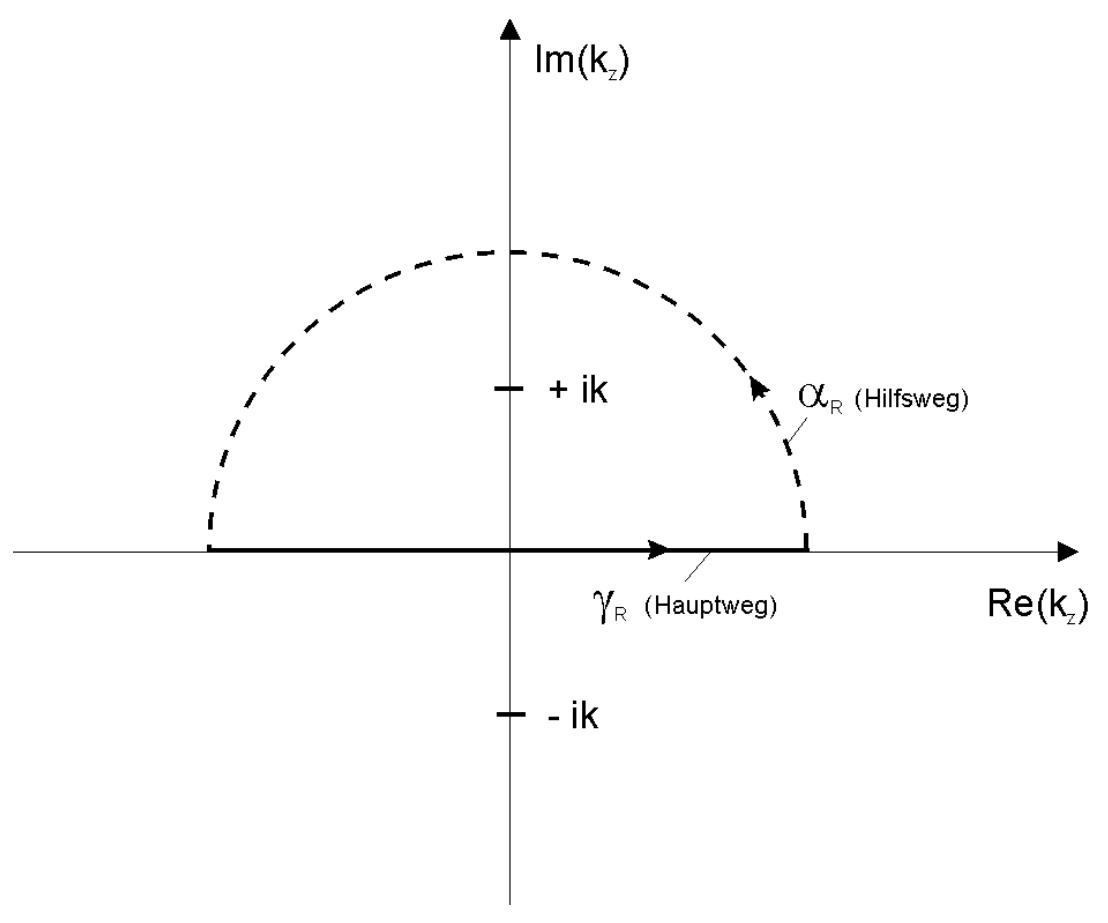

Abbildung A.1: Haupt- und Hilfsweg für die komplexe Integration in der oberen Hälfte

\section{Streufeldberechnung senkrecht aufmagnetisierter Quader}

Der Einfachheit halber wird zunächst das Streufeld einer unendlich dünnen Leiterschleife in der Höhe $z=0$ berechnet (Abb. 5.11(b)) und erst anschliessend die z-Integration ausgeführt. Es gilt die folgende Nomenklatur für den Aufpunkt und die Integrationsvariable:

$$
\vec{r}=\left(x_{0}, y_{0}, z_{0}\right) ; \quad \vec{r}^{\prime}=(x, y, 0)
$$

Somit folgt also zunächst

$$
|\vec{R}|=\left|\vec{r}-\vec{r}^{\prime}\right|=\sqrt{\left(x_{0}-x\right)^{2}+\left(y_{0}-y\right)^{2}+z_{0}^{2}} .
$$

Unter der Voraussetzung einer homogenen Magnetisierung, also eines konstanten Magnetisierungsstromes $I_{M}$ und der entsprechenden Magnetisierungsstromdichte $I_{M} \overrightarrow{d l}=\vec{\jmath}_{M} d^{3} r$ folgt nach entsprechender Parametrisierung des Randes

$$
\vec{\jmath}_{M}(r)=I_{M} \delta(z)\left[\delta(y+b) \vec{e}_{x}+\delta(x-a) \vec{e}_{y}-\delta(y-b) \vec{e}_{x}-\delta(x+a) \vec{e}_{y}\right] .
$$

Die magnetische Induktion berechnet sich aus

$$
\vec{B}(\vec{r})=\frac{\mu_{0}}{4 \pi} \int_{-a}^{a} d x \int_{-b}^{b} d y \int_{-\infty}^{\infty} d z \vec{\jmath}_{M}\left(\vec{r}^{\prime}\right) \times \frac{\vec{r}-\vec{r}^{\prime}}{\left|\vec{r}-\vec{r}^{\prime}\right|^{3}}
$$

mit $\vec{\jmath}_{M}$ aus Gl. A.14. An dieser Stelle soll nur die für die magneto-optische Messung zugängliche $\mathrm{B}_{z}(\vec{r})$-Komponente berechnet werden. Die Integration in z-Richtung ist trivial, 
da $\int_{-\infty}^{\infty} \delta(z) d z=1$ für $z=0$. Berechnen des Kreuproduktes führt auf den Vektor der magnetischen Induktion:

$$
\begin{aligned}
\vec{B}(\vec{r}) & =\frac{\mu_{0} I_{M}}{4 \pi} \int_{-a}^{a} d x \int_{-b}^{b} d y\left[\left[\delta(y+b)\left(\begin{array}{c}
0 \\
-z_{0} \\
y_{0}-y
\end{array}\right)+\delta(x-a)\left(\begin{array}{c}
z_{0} \\
0 \\
-\left(x_{0}-x\right)
\end{array}\right)\right.\right. \\
& \left.\left.-\delta(y-b)\left(\begin{array}{c}
0 \\
-z_{0} \\
y_{0}-y
\end{array}\right)-\delta(x+a)\left(\begin{array}{c}
z_{0} \\
0 \\
-\left(x_{0}-x\right)
\end{array}\right)\right] \cdot\left[\left(x_{0}-x\right)^{2}+\left(y_{0}-y\right)^{2}+z_{0}^{2}\right]^{-3 / 2}\right] .
\end{aligned}
$$

Die $B_{z}(\vec{r})$-Komponente berechnet sich dann aus

$$
B_{z}(\vec{r})=\frac{\mu_{0} I_{M}}{4 \pi} \int_{-a}^{a} d x \int_{-b}^{b} d y \frac{\left(y_{0}-y\right)[\delta(y+b)-\delta(y-b)]+\left(x_{0}-x\right)[\delta(x+a)-\delta(x-a)]}{\left[\left(x_{0}-x\right)^{2}+\left(y_{0}-y\right)^{2}+z_{0}^{2}\right]^{3 / 2}} .
$$

Nun substituiert man $\alpha=\left(x_{0}-x\right)$ und $\beta=\left(y_{0}-y\right)$, woraus entsprechend $d \alpha=-d x$ und $d \beta=-d y$ folgt. Für die Funktionen ergibt sich dann $\delta(y \pm b) \rightarrow \delta\left(y_{0}-\beta \pm b\right)$ und $\delta(x \pm a) \rightarrow \delta\left(x_{0}-\alpha \pm a\right)$, für die Grenzen $\beta_{1,2}=y_{0} \pm b$, bzw. $\alpha_{1,2}=x_{0} \pm a$. Einsetzten der neuen Integrationsvariablen und Ausführen der Integration ergibt für die $B_{z}(\vec{r})$-Komponente der unendlich dünnen Leiterschleife:

$$
\begin{aligned}
B_{z}(\vec{r}) & =\frac{\mu_{0} I_{M}}{4 \pi}\left[\left[\frac{\alpha \beta}{\left(\beta^{2}+z_{0}^{2}\right) \sqrt{\alpha^{2}+\beta^{2}+z_{0}^{2}}}\right]_{\alpha_{1}}^{\alpha_{2}}\right]_{\beta_{1}}^{\beta_{2}} \\
& +\frac{\mu_{0} I_{M}}{4 \pi}\left[\left[\frac{\alpha \beta}{\left(\alpha^{2}+z_{0}^{2}\right) \sqrt{\alpha^{2}+\beta^{2}+z_{0}^{2}}}\right]_{\alpha_{1}}^{\beta_{2}}\right]_{\beta_{1}}
\end{aligned}
$$

Nachdem nun das Streufeld der Leiterschleife bekannt ist, soll jetzt die $\mathrm{B}_{z}(\vec{r})$-Komponente eines in z-Richtung ausgedehnten Solenoiden der Dicke $d=2 h$ berechnet werden. Dazu macht man lediglich den Übergang von $z_{0} \rightarrow z_{0}-z$ und einer analogen Substitution wie oben, $\gamma=z_{0}-z$. Dies führt auf das Integral:

$$
\begin{aligned}
\left.\left.B_{z}(\vec{r})=-\frac{\mu_{0} I_{M}}{4 \pi 2 h}\left[\left[\int_{\gamma_{1}}^{\gamma_{2}} d \gamma \frac{\alpha \beta}{\left(\beta^{2}+\gamma^{2}\right) \sqrt{\alpha^{2}+\beta^{2}+\gamma^{2}}}\right]_{\alpha_{1}}^{\alpha_{2}}\right]_{\beta_{1}}^{\beta_{2}}\right]_{\alpha_{2}}^{\alpha_{2}}\right]_{\alpha_{1}}^{\beta_{2}} \\
-\frac{\mu_{0} I_{M}}{4 \pi 2 h}\left[\left[\int_{\gamma_{1}}^{\gamma_{2}} d \gamma \frac{\alpha \beta}{\left(\alpha^{2}+\gamma^{2}\right) \sqrt{\alpha^{2}+\beta^{2}+\gamma^{2}}}\right.\right.
\end{aligned}
$$

Die Lösung des Integrals ergibt sich nach einigen Umformungen mit der Remanenzpolarisation $P_{r}=\frac{\mu_{0} I_{M}}{2 h} \mathrm{zu}$

$$
B_{z}(\vec{r})=-\frac{P_{r}}{4 \pi}\left[\left[\left[\arctan \left(\frac{\gamma \sqrt{\alpha^{2}+\beta^{2}+\gamma^{2}}}{\alpha \beta}\right)\right]_{\alpha_{1}}^{\alpha_{2}}\right]_{\beta_{1}}^{\beta_{2}}\right]_{\gamma_{1}}^{\gamma_{2}} .
$$


Nach Rücktransformation erhält man durch Einsetzen der Grenzen in den Ausdruck:

$$
\begin{aligned}
B_{z}(\vec{r})=-\frac{P_{r}}{4 \pi}[ & \arctan \left(\frac{\left(z_{0}-h\right) \sqrt{\left(x_{0}-a\right)^{2}+\left(y_{0}-b\right)^{2}+\left(z_{0}-h\right)^{2}}}{\left(x_{0}-a\right)\left(y_{0}-b\right)}\right) \\
& -\arctan \left(\frac{\left(z_{0}-h\right) \sqrt{\left(x_{0}+a\right)^{2}+\left(y_{0}-b\right)^{2}+\left(z_{0}-h\right)^{2}}}{\left(x_{0}+a\right)\left(y_{0}-b\right)}\right) \\
& -\arctan \left(\frac{\left(z_{0}-h\right) \sqrt{\left(x_{0}-a\right)^{2}+\left(y_{0}+b\right)^{2}+\left(z_{0}-h\right)^{2}}}{\left(x_{0}-a\right)\left(y_{0}+b\right)}\right) \\
& +\arctan \left(\frac{\left(z_{0}-h\right) \sqrt{\left(x_{0}+a\right)^{2}+\left(y_{0}+b\right)^{2}+\left(z_{0}-h\right)^{2}}}{\left(x_{0}+a\right)\left(y_{0}+b\right)}\right) \\
& -\arctan \left(\frac{\left(z_{0}+h\right) \sqrt{\left(x_{0}+a\right)^{2}+\left(y_{0}+b\right)^{2}+\left(z_{0}+h\right)^{2}}}{\left(x_{0}+a\right)\left(y_{0}+b\right)}\right) \\
& -\arctan \left(\frac{\left(z_{0}+h\right) \sqrt{\left(x_{0}-a\right)^{2}+\left(y_{0}-b\right)^{2}+\left(z_{0}+h\right)^{2}}}{\left(x_{0}-a\right)\left(y_{0}-b\right)}\right) \\
& +\arctan \left(\frac{\left(z_{0}+h\right) \sqrt{\left(x_{0}+a\right)^{2}+\left(y_{0}-b\right)^{2}+\left(z_{0}+h\right)^{2}}}{\left(x_{0}+a\right)\left(y_{0}-b\right)}\right) \\
& +\arctan \left(\frac{\left(z_{0}+h\right) \sqrt{\left(x_{0}-a\right)^{2}+\left(y_{0}+b\right)^{2}+\left(z_{0}+h\right)^{2}}}{\left(x_{0}-a\right)\left(y_{0}-b\right)}\right)
\end{aligned}
$$

\section{Streufeldberechnung inplane aufmagnetisierter Quader}

Die Berechnung der inplane-Komponenten ist dem senkrechten Fall sehr ähnlich, deshlab werden hier nur die wichtigsten Zwischenschritte angegeben. Liege zunächst die unendlich dünne Leiterschleife in $y=0$, so ändert sich die Nomenklatur lediglich für die Integrationsvariable zu $\vec{r}^{\prime}=(x, 0, z)$. Bei Parametrisierung des Randes ergibt sich für die Stromdichte

$$
\vec{\jmath}_{M}(r)=I_{M} \delta(y)\left[\delta(z-h) \vec{e}_{x}-\delta(x-a) \vec{e}_{z}-\delta(z+h) \vec{e}_{x}+\delta(x+a) \vec{e}_{z}\right] .
$$

Für die z-Komponente der magnetischen Flussdichte folgt dann

$$
B_{z}(\vec{r})=\frac{\mu_{0} I_{M}}{4 \pi} \int_{-a}^{a} d x \int_{-h}^{h} d z \frac{y_{0}[\delta(z-h)-\delta(z+h)]}{\left[\left(x_{0}-x\right)^{2}+y_{0}^{2}+\left(z_{0}-z\right)^{2}\right]^{3 / 2}}
$$

Nun substituiert man wieder $\alpha=\left(x_{0}-x\right)$ und $\gamma=\left(z_{0}-z\right)$. Nach Integration über die beiden Substitutionsvariablen erhält man

$$
B_{z}(\vec{r})=\frac{\mu_{0} I_{M}}{4 \pi}\left[\left[\frac{\alpha y_{0}}{\left[\alpha^{2}+y_{0}^{2}+\gamma^{2}\right]^{3 / 2}\left(y_{0}^{2}+\gamma^{2}\right)}\right]_{\gamma_{1}}^{\gamma_{2}}\right]_{\alpha_{1}}^{\alpha_{2}}
$$


Für die Berechnung des Solenoiden der Breite $2 b$ macht man analog den Übergang von $y_{0} \rightarrow y_{0}-y$ mit der Substitution $\beta=y_{0}-y$. Dann folgt für die magnetische Induktion in z-Richtung nach Ausführen des Integrals mit der Remanenzpolarisation $P_{r}=\frac{\mu_{0} I_{M}}{2 b}$ :

$$
B_{z}(\vec{r})=-\frac{P_{r}}{4 \pi}\left[\left[\left[\ln \left(\sqrt{\alpha^{2}+\beta^{2}+\gamma^{2}}-\alpha\right)-\left(\sqrt{\alpha^{2}+\beta^{2}+\gamma^{2}}+\alpha\right)\right]_{\gamma_{1}}^{\gamma_{2}}\right]_{\alpha_{1}}^{\alpha_{2}}\right]_{\beta_{1}}^{\beta_{2}}
$$

Nach Rücktransformation und Einsetzen ergibt sich für den Fall einer reinen inplaneMagnetisierung die exakte Lösung der z-Komponente des Streufeldes zu

$$
\begin{aligned}
& B_{z}(\vec{r})=-\frac{P_{r}}{4 \pi}\left[\ln \left(\sqrt{\left(x_{0}-a\right)^{2}+\left(y_{0}-b\right)^{2}+\left(z_{0}-h\right)^{2}}-\left(x_{0}-a\right)\right)\right. \\
& -\ln \left(\sqrt{\left(x_{0}-a\right)^{2}+\left(y_{0}-b\right)^{2}+\left(z_{0}-h\right)^{2}}+\left(x_{0}-a\right)\right) \\
& -\ln \left(\sqrt{\left(x_{0}+a\right)^{2}+\left(y_{0}-b\right)^{2}+\left(z_{0}-h\right)^{2}}-\left(x_{0}+a\right)\right) \\
& +\ln \left(\sqrt{\left(x_{0}+a\right)^{2}+\left(y_{0}-b\right)^{2}+\left(z_{0}-h\right)^{2}}+\left(x_{0}+a\right)\right) \\
& -\ln \left(\sqrt{\left(x_{0}-a\right)^{2}+\left(y_{0}+b\right)^{2}+\left(z_{0}-h\right)^{2}}-\left(x_{0}-a\right)\right) \\
& +\ln \left(\sqrt{\left(x_{0}-a\right)^{2}+\left(y_{0}+b\right)^{2}+\left(z_{0}-h\right)^{2}}+\left(x_{0}-a\right)\right) \\
& +\ln \left(\sqrt{\left(x_{0}+a\right)^{2}+\left(y_{0}+b\right)^{2}+\left(z_{0}-h\right)^{2}}-\left(x_{0}+a\right)\right) \\
& -\ln \left(\sqrt{\left(x_{0}+a\right)^{2}+\left(y_{0}+b\right)^{2}+\left(z_{0}-h\right)^{2}}+\left(x_{0}+a\right)\right) \\
& -\ln \left(\sqrt{\left(x_{0}-a\right)^{2}+\left(y_{0}-b\right)^{2}+\left(z_{0}+h\right)^{2}}-\left(x_{0}-a\right)\right) \\
& +\ln \left(\sqrt{\left(x_{0}-a\right)^{2}+\left(y_{0}-b\right)^{2}+\left(z_{0}+h\right)^{2}}+\left(x_{0}-a\right)\right) \\
& +\ln \left(\sqrt{\left(x_{0}+a\right)^{2}+\left(y_{0}-b\right)^{2}+\left(z_{0}+h\right)^{2}}-\left(x_{0}+a\right)\right) \\
& -\ln \left(\sqrt{\left(x_{0}+a\right)^{2}+\left(y_{0}-b\right)^{2}+\left(z_{0}+h\right)^{2}}+\left(x_{0}+a\right)\right) \\
& +\ln \left(\sqrt{\left(x_{0}-a\right)^{2}+\left(y_{0}+b\right)^{2}+\left(z_{0}+h\right)^{2}}-\left(x_{0}-a\right)\right) \\
& -\ln \left(\sqrt{\left(x_{0}-a\right)^{2}+\left(y_{0}+b\right)^{2}+\left(z_{0}+h\right)^{2}}+\left(x_{0}-a\right)\right) \\
& -\ln \left(\sqrt{\left(x_{0}+a\right)^{2}+\left(y_{0}+b\right)^{2}+\left(z_{0}+h\right)^{2}}-\left(x_{0}+a\right)\right) \\
& \left.+\ln \left(\sqrt{\left(x_{0}+a\right)^{2}+\left(y_{0}+b\right)^{2}+\left(z_{0}+h\right)^{2}}+\left(x_{0}+a\right)\right) \cdot\right]
\end{aligned}
$$




\section{Literaturverzeichnis}

[1] Albrecht, M. ; Rettner, C.T. ; Moser, A. ; Best, M.E. ; Terris, B.D.: Recording performance of high-density patterned perpendicular magnetic media. In: Appl. Phys. Lett. 81 (2002), Nr. 15, S. 2875

[2] Koike, K. ; Matsuyama, H. ; Hirayama, Y. ; Tanahashi, K. ; Kanemura, T. ; Kitakami, O. ; Shimada, Y.: Magnetic block array for patterned magnetic media. In: Appl. Phys. Lett. 78 (2001), Nr. 6, S. 784

[3] Ross, C.A.: Patterned Magnetic Recording Media. In: Ann. Rev. Mater. Res. 31 (2001), S. 203-235

[4] Kitade, Y. ; Komoriya, H. ; Maruyama, T.: Patterned Media Fabricated by Lithography and Argon-Ion Milling. In: IEEE Trans. Magn. 40 (2004), Nr. 4, S. 2516

[5] Ross, C.A. ; Smith, H.I. ; Savas, T. ; Schattenburg, M. ; Farhoud, M. ; Hwang, M. ; Walsh, M. ; Abraham, M.C. ; Ram, R.J.: Fabrication of patterned media for high density magnetic storage. In: J. Vac. Sci. Technol. B 17 (1999), Nr. 6, S. 3168

[6] Johnson, M.: Hybrid ferromagnet-semiconductor devices. In: J. Vac. Sci. Technol. A 16 (1998), S. 1806

[7] Martin, J.I. ; Nogues, J. ; Liu, K. ; Vicent, J.L. ; Schuller, I.: Ordered magnetic nanostructures: fabrication and properties. In: J. Magn. Magn. Mater. 256 (2003), S. 449-501

[8] Cowburn, R.P. ; Welland, M.E.: Room Temperature Magnetic Quantum Cellular Automata. In: Science 287 (2000), S. 1466

[9] Schandt, D.M. ; Yu, E.T. ; Sankar, S. ; Berkowitz, A.E.: Characterization and analysis of a novel hybrid magnetoelectronic device for magnetic field sensing. In: J. Vac. Sci. Technol. A 18 (2000), Nr. 4, S. 1834

[10] DeGraef, M. ; Zhu, Y.: Magnetic Imaging and its Applications to Materials. In: R. Celotta and T. Lucatorto Experimental Methods in the Physical Sciences. Academic Press, 2001

[11] Hopster, H. ; Oepen, H.P.: Magnetic Microscopy of Nanostructures. Springer Verlag, 2005 
[12] Cowburn, R.P.: Property variation with shape in magnetic nanoelements. In: $J$. Phys. D: Appl. Phys. 33 (2000), S. R1-R16

[13] Cowburn, R.P.: Magnetic nanodots for device application. In: J. Magn. Magn. Mater. 242-245 (2002), S. 505-511

[14] Cowburn, R.P. ; Adeyeye, A.O. ; Welland, M.E.: Controlling magnetic ordering in coupled nanomagnet arrays. In: New Journal of Physics 1 (1999), S. $16.1-16.9$

[15] Aign, T. ; P.Meyer ; Lemerle, S. ; Jamet, J.P. ; Ferre, J. ; Mathet, V. ; Chappert, C. ; Gierak, J. ; Vieu, C. ; Rousseaux, F. ; Launois, H. ; Bernas, H.: Magnetization Reversal in Arrays of Perpendicular Magnetized Ultrathin Dots Coupled by Dipolar Interaction. In: Phys. Rev. Lett. 81 (1998), Nr. 25, S. 5656

[16] Hwang, M. ; Abraham, M.C. ; Savas, T.A. ; Smith, H.I. ; Ram, R.J. ; Ross, C.A.: Magnetic force microscopy study of interactions in $100 \mathrm{~nm}$ period nanomagnet arrays. In: J. Appl. Phys. 87 (2000), Nr. 9, S. 5108

[17] Zhang, L.F. ; Xu, C. ; Hui, P.M. ; MA, Y.Q.: Influence of dipolar interactions on small magnetic dot arrays. In: J. Appl. Phys. 97 (2005), S. 103912

[18] Vavassori, P. ; Bisero, D. ; Carace, F. ; Liberati, M. ; Bona, A. di ; Gazzadi, G.C. ; VALERI, S.: Magnetocrystalline and configurational anisotropies in Fe nanostructures. In: J. Magn. Magn. Mater. 290 (2005), S. 183-186

[19] Jooss, Ch.: Verankerungsmechanismen von Flußlinien in YBaCuO-Schichten. Habilitationsschrift, Cuvillier Verlag Göttingen, 1998

[20] Jooss, C. ; Albrecht, J. ; Kuhn, H. ; Leonhardt, S. ; Kronmüller, H.: Magneto-optical studies of current distributions in high $\mathrm{T}_{c}$ superconductors. In: Rep. Prog. Phys. 65 (2002), S. 651-788

[21] Jooss, C. ; Warthmann, R. ; Forkl, A. ; Kronmüller, H.: High-resolution magneto-optical imaging of critical currents in $\mathrm{YBa}_{2} \mathrm{Cu}_{3} \mathrm{O}_{7-\delta}$ thin films. In: Physica C 299 (1998), S. 215-230

[22] Dorosinskit, L.A. ; Indenbom, M.V. ; Nikitenko, V.I. ; Ossip'yan, Y.A. ; Polyanski, A.A. ; Vlasko-Vlasov, V.K.: Studies of HTSC crystal magnetization features using indicator magnetooptic films with in-plane anisotropy. In: Physica $C$ 203 (1992), S. 149-156

[23] Johansen, T.H. ; Shantsev, D.V.: Magneto-optical Imaging. Bd. 142. Kluwer Academic Publishers, Nato Science Series, II. Mathematics, Physics and Chemistry, 2004

[24] Pannetier, M. ; Klassen, F.C. ; Wijngaarden, R.J. ; Welling, M. ; Heeck, K. ; Huijbregtse, J.M. ; Dam, B. ; Griessen, R.: Magneto-optical investigation of flux penetration in a superconducting ring. In: Phys. Rev. B 64 (2001), S. 144505 
[25] Zvezdin, A.K. ; Kotov, V.A.: Modern Magnetooptics and Magnetooptical Materials. Institute of Physics Publishing Bristol and Philadelphia, 1997 (Studies in Condensed Matter Physics)

[26] Bennett, L.H. ; McMichael, R.D. ; Swartzendruber, L.J. ; Hua, S. ; Lashmore, D.S. ; Shapiro, A.J. ; Gornakov, V.S. ; Dedukh, L.M. ; Nikitenko, V.I.: Magneto-optical indicator film observation of domain structure in magnetic multilayers. In: Appl. Phys. Lett. 66 (1995), Nr. 7, S. 888

[27] Kabanov, Yu. ; Zhukov, A. ; Zhukova, V. ; Gonzales, J.: Magnetic domain structure of wires studied by using the magneto-optical indicator film method. In: Appl. Phys. Lett. 87 (2005), S. 142507

[28] Jiang, J.S. ; Bader, S.D. ; Kaper, H. ; Leaf, G.K. ; Shull, R.D. ; Shapiro, A.J. ; Gornakov, V.S. ; Nikitenko, V.I. ; Platt, C.L. ; Berkowitz, A.E. ; DAVID, S. ; Fullerton, E.E.: Rotational hysteresis of exchange-spring magnets. In: J. Phys. D 35 (2002), S. 2339-2343

[29] Khapikov, A. ; Uspenskaya, L. ; Bdikin, I. ; Mukovskit, Y. ; Karabashev, S. ; Shulyaev, D. ; Arsenov, A.: Magnetic domains and twin structure of the $\mathrm{La}_{0.7} \mathrm{Sr}_{0.3} \mathrm{MnO}_{3}$ single crystal. In: Appl. Phys. Lett. 77 (2000), Nr. 15, S. 2376

[30] Gornakov, V.S. ; Nikitenko, V.I. ; Bennett, L.H. ; Brown, H.J. ; Donahue, M.J. ; Egelhoff, W.F. ; McMichael, R.D. ; Shapiro, A.J.: Experimental study of magnetization reversal processes in nonsymmetric spin valve. In: J. Appl. Phys. 81 (1997), Nr. 8, S. 5215

[31] Nikitenko, V.I. ; Gornakov, V.S. ; Dedukh, L.M. ; Khapikov, A.F. ; BenNetT, L.H. ; McMichael, R.D. ; Swartzendruber, L.J. ; Shapiro, A.J. ; Donahue, M.J. ; Matveev, V.N. ; Levashov, V.I.: Magneto-optical indicator film (MOIF) microscopy of granular and layer structures. In: J. Appl. Phys. 79 (1996), Nr. 8, S. 6073

[32] Williams, H.J. ; Foster, F.G. ; E.A.Wood: Observation of Magnetic Domains by the Kerr Effect. In: Phys. Rev. 82 (1951), S. 119

[33] Cebollada, A. ; Weller, D. ; Sticht, J. ; Harp, G.R. ; Farrow, R.F.C. ; Marks, R.F. ; Savoy, R. ; Scott, J.C.: Enhanced magneto-optical Kerr effect in spontanously ordered FePt alloy: Quantitative agreement between theory and experiment. In: Phys. Rev. B 50 (1994), Nr. 5, S. 3419

[34] Hubert, A. ; Schäfer, R.: Magnetic Domains. Springer Verlag, 2000

[35] McCord, J.; Westwood, J.: Domain formation in Fe-N multilayers. In: J. Appl. Phys. 87 (2000), Nr. 9, S. 6502

[36] Fumagalli, P. ; Rosenberger, A. ; Eggers, E. ; Münnemann, A. ; Held, N. ; Güntherodt, G.: Quantitative determination of the local Kerr rotation by scanning near-field magneto-optical microscopy. In: Appl. Phys. Lett. 72 (1998), Nr. 22, S. 2803 
[37] Hug, H.J. ; Stiefel, B. ; Schendel, P.J.A. van ; Moser, A. ; Hofer, R. ; Martin, S. ; Güntherodt, H.-J. ; Porthun, S. ; Abelmann, L. ; Lodder, J.C. ; Bochi, G. ; O'Handley, R.C.: Quantitative magnetic force microscopy on perpendicularly magnetized samples. In: J. Appl. Phys. 83 (1998), Nr. 11, S. 5609

[38] Schendel, P.J.A. van ; Hug, H.J. ; Stiefel, B. ; Martin, S. ; Güntherodt, H.-J.: A method for the calibration of magnetic force microscopy tips. In: J. Appl. Phys. 88 (2000), Nr. 1, S. 435

[39] Dreyer, S. ; Norpoth, J. ; Jooss, C. ; Sievers, S. ; Siegner, U. ; Neu, V. ; JoHANSEN, T.H.: Quantitative imaging of stray fields and magnetization distributions in hard magnetic element arrays. In: J. Appl. Phys. 101 (2007), S. 083905

[40] Hong, M.H. ; Hono, K. ; Watanabe, M.: Microstructure of FePt/Pt magnetic thin films with high perpendicular coercivity. In: J. Appl. Phys. 84 (1998), Nr. 8, S. 4403

[41] Thiele, J.-U. ; Folks, L. ; Toney, M.F. ; Weller, D.K.: Perpendicular magnetic anisotropy and magnetic domain structure in sputtered epitaxial FePt (001) L1 0 films. In: J. Appl. Phys. 84 (1998), Nr. 10, S. 5686

[42] Visokay, M.R. ; Sinclair, R.: Direct formation of ordered CoPt and FePt compound thin films by sputtering. In: Appl. Phys. Lett. 66 (1995), Nr. 13, S. 1692

[43] Norpoth, J.: Modifikation der magnetostatischen Wechselwirkung in hartmagnetischen Elementen durch künstliche Kantenrauhigkeit, Universität Göttingen, Diplomarbeit, 2006

[44] Patra, A.K. ; Neu, V. ; Fähler, S. ; Wendrock, H. ; Schultz, L.: Phase formation, texture, and magnetic properties of epitaxial Pr-Co films grown on $\mathrm{MgO}(100)$. In: J. Appl. Phys. 100 (2006), S. 043905

[45] Sievers, S. ; Albrecht, M. ; Siegner, U. ; Dreyer, S. ; Jooss, C.: Combined optical and force microscopy of patterned magnetic films. In: J. Appl. Phys. 99 (2006), S. 08S504

[46] Schnittger, S. ; Dreyer, S. ; Jooss, C. ; Sievers, S. ; Siegner, U.: Magnetostatic interactions in patterned $\mathrm{CoPt}$ films embedded in a permalloy matrix. In: Appl. Phys. Lett. 90 (2007)

[47] Greiner, W.: Klassische Elektrodynamik. Wissenschaftlicher Verlag Harri Deutsch, 2002

[48] Kapitel 5. In: JACKSON, J.D.: Klassische Elektrodynamik. Walter De Gruyter Verlag, 2002

[49] Kapitel 9. In: Grossmann, S.: Mathematischer Einführungskurs für die Physik. B. G. Teubner Stuttgart (Teubner Studienbücher: Physik), 1993

[50] Rave, W. ; Fabian, K. ; Hubert, A.: Magnetic states of small cubic particles with uniaxial anisotropy. In: J. Magn. Magn. Mater. 190 (1998), S. 332-348 
[51] Mayergoyz, I.D. ; Adly, A.A. ; Gomez, R.D. ; Burke, E.R.: Magnetization image reconstruction from magnetic force scanning tunneling microscopy images. In: J. Appl. Phys. 73 (1993), Nr. 10, S. 5799

[52] Hsu, C.-C. ; Miller, C.T. ; Indeck, R.S. ; O'Sullivan, J.A. ; Muller, M.W.: Magnetization Estimation From MFM Images. In: IEEE Trans. Magn. 38 (2002), Nr. 5, S. 2444

[53] Feldmann, D.M.: Resolution of two-dimensional currents in superconductors from a two-dimensional magnetic field measurement by the method of regularization. In: Phys. Rev. B 69 (2004), S. 144515

[54] Mayergoyz, I.D. ; D'Angelo, J.D. ; Crowley, C.: Solution of three-dimensional boundary value problems for magnetostatics by a constrained Fredholm integral equation. In: J. Appl. Phys. 57 (1985), Nr. 1, S. 3832

[55] Ciulli, S. ; Pidcock, M.K. ; Sebu, C.: An integral equation method for the inverse conductivity problem. In: Physics Letters A 325 (2004), S. 253-267

[56] Louis, A.K.: Medical imaging: state of the art and future development. In: Inverse Problems 8 (1992), S. 709-738

[57] Zhao, T. ; Fujiwara, H. ; G.J.Mankey ; Hou, C. ; Sun, M.: Reconstruction of in-plane magnetization distributions from magnetic force microscope images. In: $J$. Appl. Phys. 89 (2001), Nr. 11, S. 7230

[58] Beardsley, I.A.: Reconstruction of the Magnetization in a Thin Film by a Combination of Lorentz Microscopy and External Field Measurements. In: IEEE Trans. Magn. 25 (1989), Nr. 1, S. 671

[59] Mansuripur, M.: Computation of electron diffraction patterns in Lorentz electron microscopy of thin magnetic films. In: J. Appl. Phys. 69 (1991), Nr. 15, S. 2455

[60] Schönenberger, C. ; Alvarado, S.F.: Understanding magnetic force microscopy. In: Z. Phys. B - Condensed Matter 80 (1990), S. 373-383

[61] Burke, E.R. ; Gomez, R.D. ; Madabhushi, R. ; Mayergoyz, I.D.: Computation of magnetic fields from recording surfaces with multiple tracks. In: J. Appl. Phys. 75 (1991), Nr. 10, S. 5759

[62] Engström, S.: Green Function Method for Calculating Properties of Static Magnetic Fields. In: Bioelectromagnetics 22 (2001), S. 511-518

[63] Hoffmann, J.: Präparation und Untersuchung der supraleitenden Eigenschaften von metastabilen NbAl-Dünnfilmen, Inst. f. Materialphysik, Universität Göttingen, Diplomarbeit, 1991

[64] Singh, A. ; Tamm, R. ; Neu, V. ; Fähler, S. ; Oertel, C.-G. ; Skrotzki, W. ; Schultz, L. ; Holzapfel, B.: Epitaxial growth of highly coercive Sm-Co thin films using pulsed laser deposition. In: J. Appl. Phys. 97 (2005), S. 093902 
[65] Krebs, H.-U.: Laser deposition of metallic alloys and multilayers. In: Appl. Surf. Sci. 86 (1995)

[66] Chrisey, D. B. ; Hubler, G. K.: Pulsed Laser Deposition Of Thin Films. WileyInterscience Publication, 1994

[67] Wolf, S. ; Tauber, R.: Silicon Processing for the VLSI Era. Lattice Press, Ca., 1986

[68] Cullity, B. D. ; Stock, S. R.: Elements of X-Ray Diffraction. Prentice Hall, 2001

[69] ThIELE, K.: Ionenstrahlunterstütztes Wachstum von Zinn-dotierten IndiumoxidFilmen, Inst. f. Materialphysik, Universität Göttingen, Diss., 2004

[70] DzICK, J.: Mechanismen der ionenstrahlunterstützten Texturbildung in YSZ-Filmen, Inst. f. Materialphysik, Universität Göttingen, Diss., 2000

[71] Warren, B.E.: X-Ray Diffraction. Addison-Wesley, Reading, Massachusetts, 1969

[72] SAgeL, K.: Tabellen zur Röntgenanalyse. Springer Verlag, 1958

[73] Giessibl, F.J.: Advances in atomic force microscopy. In: Rev. Mod. Phys. 75 (2003), Nr. 3, S. 949

[74] Hartmann, U.: Magnetic Force Microscopy. In: Annu. Rev. Mater. Sci. 29 (1999)

[75] Grütter, P. ; Wadas, A. ; Meyer, E. ; Heinzelmann, H. ; Hidber, H.-R. ; GüntherodT, H.-J.: High resolution magnetic force microscopy. In: J. Vac. Sci. Technol. A 8 (1989), Nr. 1, S. 406

[76] Hartmann, U.: The point dipole approximation in magnetic force microscopy. In: Physics Letters A 137 (1989), Nr. 9, S. 475

[77] Kong, L. ; Chou, S.Y.: Quantification of magnetic force microscopy using a micronscale current ring. In: Appl. Phys. Lett. 70 (1997), Nr. 15, S. 2043

[78] Liu, C. ; Lin, K. ; Holmes, R. ; Mankey, G. ; Fujiwara, H. ; Jiang, H. ; Cho, H.S.: Calibration of magnetic force microscopy using micron size straight current wires. In: J. Appl. Phys. 91 (2002), Nr. 10, S. 8849

[79] KeBe, Th. ; CARL, A.: Calibration of magnetic force microscopy tips by using nanoscale current-carrying parallel wires. In: J. Appl. Phys. 95 (2004), Nr. 3, S. 775

[80] Landis, S. ; RodmacQ, B. ; Dieny, B.: Fabrication of submicron magnetic line arrays for magnetic force microscopy tip calibration. In: Microelectronic Engineering 73-74 (2004), S. 790-796

[81] Lohau, J. ; Kirsch, S. ; Carl, A. ; Dumpich, G. ; Wassermann, E.F.: Quantitative determination of effective dipole and monopole moments of magnetic force microscopy tips. In: J. Appl. Phys. 86 (1999), Nr. 6, S. 3410 
[82] Matteucci, G. ; Frost, B.G. ; Medina, F.F.: Study of the field around magnetic force microscopy probes using electron holography. In: Ultramicroscopy 99 (2004), Nr. 2-3, S. 95-102

[83] JiLES, D.: Introduction to Magnetism and Magnetic Materials. Chapmann and Hall - CRC Press company, 1998

[84] Bracchi, A.: Structural and Magnetic Properties of the Glass-Forming Alloy $N d_{60} F_{30} A l_{10}$, Georg-August-Universität Göttingen, Diss., 2004

[85] Massalski, T.B. ; Okamoto, H. ; Subramanian, P.R. ; Kacprzak, L.: Binary Alloy Phase Diagrams. 2nd. ASM International, 1990

[86] Окамото, H.: Phase Diagrams of Binary Iron Alloys. ASM Int., 1993

[87] Seki, T. ; Shima, T. ; Yakushiji, K. ; Takanashi, K.: Dot size dependence of magnetic properties in microfabricated $\mathrm{L} 1_{0}-\mathrm{FePt}(001)$ and $\mathrm{L} 1_{0}-\mathrm{FePt}$ (110) dot arrays. In: J. Appl. Phys. 100 (2006), S. 043915

[88] Goto, T. ; Ide, Y. ; Abe, H. ; Watanabe, K. ; Onagawa, J. ; Yoshida, H. ; CADOGAN, J.M.: Magnetization process of epitaxial FePt film with granular structure. In: J. Magn. Magn. Mater. 198-199 (1999), S. 486-488

[89] Okamoto, S. ; Kikuchi, N. ; Kitakami, O. ; Miyazaki, T. ; Shimada, Y.: Chemical-order-dependent magnetic anisotropy and exchange stiffness constant of FePt (001) epitaxial films. In: Phys. Rev. B 66 (2002), S. 024413

[90] Lim, B.C. ; Chen, J.S. ; Wang, J.P.: Thickness dependence of structural and magnetic properties of FePt films. In: J. Magn. Magn. Mater. 271 (2004), S. 159164

[91] Barmak, K. ; Kim, J. ; Lewis, L.H. ; Coffey, K.R. ; Toney, M.F. ; KelLOCK, A.J. ; ThIELE, J.-U.: Stoichiometry-anisotropy connections in epitaxial L1 $1_{0}$ FePt(001) films. In: J. Appl. Phys. 95 (2004), Nr. 11, S. 7501

[92] Kanazawa, H. ; Lauhoff, G. ; Suzuki, T.: Magnetic and structural properties of $\left(\mathrm{Co}_{x} \mathrm{Fe}_{100-x}\right)_{50} \mathrm{Pt}_{50}$ alloy thin films. In: J. Appl. Phys. 87 (2000), Nr. 9, S. 6143

[93] Farrow, R.F.C. ; Weller, D. ; Marks, R.F. ; Toney, M.F. ; Hom, S. ; Harp, G.R. ; Cebollada, A.: Growth temperature dependence of long-range alloy order and magnetic properties of epitaxial $\mathrm{Fe}_{x} \mathrm{Pt}_{1-x}(x \approx 0.5)$ films. In: Appl. Phys. Lett. 69 (1996), Nr. 8, S. 1166

[94] Zhang, Y. ; Wan, J. ; Skumryev, V. ; Stoyanov, S. ; Huang, Y. ; HadjiPANAYIS, G.C.: Microstructural characterization of $\mathrm{L}_{0}$ FePt/MgO nanoparticles with perpendicular anisotropy. In: Appl. Phys. Lett. 85 (2004), Nr. 22, S. 5343

[95] Zhao, Z.L. ; Chen, J.S. ; Ding, J. ; Yi, J.B. ; LiU, B.H. ; Wang, J.P.: Fabrication and microstructure of high coercivity FePt thin films at $400{ }^{\circ} \mathrm{C}$. In: Appl. Phys. Lett. 88 (2006), S. 052503 
[96] HaAsen, P.: Physikalische Metallkunde. Springer Verlag, 1994

[97] Hansen, M. ; Anderko, K.: Constitution of Binary Alloys. New York: McGrawHill Book Comp., 1958

[98] Gebhardt, E. ; Köster, W.: In: Z. Metallkunde 32 (1940)

[99] Barmak, K. ; Kim, J. ; Lewis, L.H. ; Coffey, K.R. ; Toney, M.F. ; Kellock, A.J. ; ThIELE, J.-U.: On the relationship of magnetocrystalline anisotropy and stoichiometry in epitaxial L1 ${ }_{0} \mathrm{CoPt}$ (001) and FePt (001) thin films. In: J. Appl. Phys. 98 (2005), S. 033904

[100] Sun, X. ; Jia, Z.Y. ; Huang, Y.H. ; Harrell, J.W. ; Nikles, D.E. ; Sun, K. ; WAng, L.M.: Synthesis and magnetic properties of CoPt nanoparticles. In: J. Appl. Phys. 95 (2004), Nr. 11, S. 6747

[101] Yamaguchi, K. ; Zhao, Z. ; Chen, C. ; Hashimoto, M. ; Shi, J. ; Nakamura, Y.: Structure and magnetic behavior of $\mathrm{CoPt}$ films prepared on $\mathrm{MgO}(001)$ substrates by dc plasma biased sputtering. In: Thin Solid Films 459 (2004), S. 165-168

[102] O’Handley, R.C.: Modern Magnetic Materials - Principles and Applications. Wiley-Interscience Publication, 2000

[103] Ersen, O. ; Parasote, V. ; Pierron-Bohnes, V. ; Cadeville, M.C. ; UlhaqBouillet, C.: Growth conditions to optimize chemical order and magnetic properties in molecular-beam-epitaxy-grown $\mathrm{CoPt} / \mathrm{MgO}(001)$ thin films. In: J. Appl. Phys. 93 (2003), Nr. 5, S. 2987

[104] Shima, T. ; Takanashi, K. ; Takahashi, Y.K. ; Hono, K.: Preparation and magnetic properties of highly coercive FePt films. In: Appl. Phys. Lett. 81 (2002), Nr. 6, S. 1050

[105] Shima, T. ; Takanashi, K. ; Takahashi, Y.K. ; Hono, K. ; Li, G.Q. ; Ishio, S.: High coercivity and magnetic domain observation in epitaxially grown particulate FePt thin films. In: J. Magn. Magn. Mater. 266 (2003), S. 171-177

[106] Chen, Z. ; Meng-Burany, X. ; Hadjipanayis, G.C.: High coercivity in nanostructured $\mathrm{PrCo}_{5}$-based powders produced by mechanical milling and subsequent annealing. In: Appl. Phys. Lett. 75 (1999), Nr. 20, S. 3165

[107] Keavney, D.J. ; Fullerton, E.E. ; Li, D. ; Sowers, C.H. ; Bader, S.D.: Enhanced Co orbital moments in Co-rare-earth permanent-magnet films. In: Phys. Rev. B 57 (1998), Nr. 9, S. 5291

[108] Malhotra, S.S. ; Liu, Y. ; Shan, Z.S. ; Liou, S.H. ; Stafford, D.C. ; Sellmyer, D.J.: Nanocrystalline high coercivity $\mathrm{PrCo} / \mathrm{Cr}$ thin films: potential high density magnetic recording media. In: J. Magn. Magn. Mater. 161 (1995), S. 316-322

[109] HüBener, R.P.: Magnetic flux structures in superconductors. Bd. Springer series in solid state sciences, vol. 6. Springer, Berlin, 1979 
[110] Hagedorn, O.: Herstellung und Charakterisierung magnetischer Granatfilme für die magnetooptische Sensorik, Universität Osnabrück, Diss., März 2002

[111] KlanK, M.: Entwicklung bildgebender magnetooptischer Sensoren zur Visualisierung magnetischer Felder, Universität Osnabrück, Diss., März 2002

[112] Aichele, T. ; Lorenz, A. ; Hergt, R. ; Görnert, P.: Garnet layers prepared by liquid phase epitaxy for microwave and magneto-optical applications - a review. In: Cryst. Res. Technol. 38 (2003), Nr. 7-8, S. 575-587

[113] Grechishkin, R.M. ; Goosev, M.Y. ; Ilyashenko, S.E. ; Neustroev, N.S.: High resolution sensitive magneto-optic ferrite-garnet films with planar anisotropy. In: J. Magn. Magn. Mater. 157/158 (1996), S. 305-306

[114] Adachi, N. ; Denysenkov, V.P. ; Khartsev, S.I. ; Grishin, A.M.: Epitaxial $\mathrm{Bi}_{3} \mathrm{Fe}_{5} \mathrm{O}_{12}(001)$ films grown by pulsed laser deposition and reactive ion beam sputtering techniques. In: J. Appl. Phys. 88 (2000), Nr. 5, S. 2734

[115] Wijngaarden, R.J. ; Heeck, K. ; Welling, M. ; Limburg, R. ; Pannetier, M. ; Zetten, K. van ; Roorda, V.L.G. ; Voorwinden, A.R.: Fast imaging polarimeter for magneto-optical investigations. In: Rev. Sci. Instrum. 72 (2001), Nr. 6, S. 2661

[116] Wilhelm, S. ; Gröbeler, B. ; Gluch, M. ; Heinz, H.: Die konfokale Laser Scanning Mikroskopie, Grundlagen / Carl Zeiss Jena GmbH. - Forschungsbericht

[117] Janke, I.: Anwendungen von Weitfeldmikroskopie mit strukturierter Beleuchtung für funktionelle Hirnabbildung, Ruprecht- Karls-Universität Heidelberg, Diss., 2004

[118] Vogel, H.: Gerthsen Physik. Springer, 1995

[119] Vollrath, W.: Optische Mikroskopie bei einer Auflösung unter 0,1 $\mu m$ : Fiktion oder Vision. In: Mitteilungen für Wissenschaft und Technik Ausgabe CDR 2 (April 1999), S. 49-70

[120] BoRn, V.: Mechanismen der Vortexdynamik im Hochtemperatursupraleiter $\mathrm{YBa}_{2} \mathrm{Cu}_{3} \mathrm{O}_{7-x}$, Institut f. Materialphysik, Universität Göttingen, Diss., 2004

[121] Guth, C.: Mechanismen der Stromunterdrückung in supraleitenden $Y B a_{2} C u_{3} O_{7-\delta}$ Kleinwinkelkorngrenzen, Institut f. Materialphysik, Universität Göttingen, Diss., 2004

[122] Dhagat, P. ; Glavinas, E. ; Jander, A. ; Porter, D.G. ; Indeck, R.S. ; MulLER, M.W.: Track-width dependence of transition jitter. In: J. Appl. Phys. 79 (1996), Nr. 8, S. 5652

[123] Porter, D.G. ; Glavinas, E. ; Dhagat, P. ; O’Sullivan, J.A. ; Indeck, R.S. ; Muller, M.W.: Irregular grain structure in micromagnetic simulations. In: $J$. Appl. Phys. 79 (1996), Nr. 8, S. 4695

[124] Miles, J.J. ; Middleton, B.K.: The Role of Microstructure in Micromagnetic Models of Longitudinal Thin Film Media. In: IEEE Trans. Magn. 26 (1990), S. 2137 
[125] Miles, J.J. ; Wdowin, M. ; OAkley, J. ; Middleton, B.K.: The Effect of Cluster Size on Thin Film Media Noise. In: IEEE Trans. Magn. 31 (1995), S. 1013

[126] WadhwA, P. ; JALIL, M.B.A.: Micromagnetic modeling and analysis of linear array of square nanomagnets. In: J. Magn. Magn. Mater. 294 (2005), S. 83-89

[127] Miramond, C. ; Fermon, C. ; Roussaux, F. ; Decanini, D. ; Carcenac, F.: Permalloy cylindrical submicron size dot arrays. In: J. Magn. Magn. Mater. 165 (1997), S. 500-503

[128] Belashenko, K.D.: Anisotropy of exchange stiffness and its effect on the properties of magnets. In: J. Magn. Magn. Mater. 270 (2004), S. 413-424

[129] Brown, W.F.: Magnetostatic Principles in Ferromagnetism. In: E.P. Wohlfarth Selected Topics in Solid State Physics. North-Holland, Amsterdam, 1962

[130] Mathieu, C.: Brillouin-Lichtstreuuntersuchungen an strukturierten magnetischen Systemen, Universität Kaiserslautern, Diss., 1998

[131] Osborn, J.A.: Demagnetizing Factors of the General Ellipsoid. In: Physical Review 67 (1945), Nr. 11-12, S. 351

[132] Norpoth, J. ; Dreyer, S. ; Jooss, C.: Manipulating the dipolar magnetic interactions in FePt square arrays: The role of edge roughness. In: J. Appl. Phys. 101 (2007), S. 09F518

[133] Persönliche Mitteilungen Jonas Norpoth: Berechnung des Entmagnetisierungsfeldes der CoPt-Strukturen

[134] KIM, M.-G. ; ShIn, S.-C.: Ordering of island-like FePt $L 1_{0}$ thin films. In: Appl. Phys. Lett. 80 (2002), Nr. 20, S. 3802

[135] Ristau, R.A. ; Barmak, K. ; Lewis, L.H. ; Coffey, K.R. ; Howard, J.K.: On the relationship of high coercivity and $L 1_{0}$ ordered phase in CoPt and FePt thin films. In: J. Appl. Phys. 86 (1999), Nr. 8, S. 4527

[136] Karanasos, V. ; Panagiotopoulos, I. ; Niarchos, D.: CoPt:B granular thin films for high density magnetic recording media. In: J. Magn. Magn. Mater. 236 (2001), S. 2740

[137] Skomski, R. ; Coey, J.M.D.: Giant energy product in nanostructured two-phase magnets. In: Phys. Rev. B 48 (1993), Nr. 21, S. 15812

[138] Li, G. ; Takahoshi, H. ; Ito, H. ; Washiya, T. ; Saito, H. ; Ishio, S. ; Shima, T. ; TAKanashi, K.: Mechanism of magnetization process of island-like $\mathrm{L} 1_{0} \mathrm{FePt}$ films. In: J. Magn. Magn. Mater. 287 (2005), S. 219-223

[139] Okamoto, S. ; Kitakami, O. ; Kikuchi, N. ; Miyazaki, T. ; Shimada, Y.: Size dependences of magnetic properties and switching behavior in $\mathrm{FePt} \mathrm{L}_{0}$ nanoparticles. In: Phys. Rev. B 67 (2003), S. 094422 


\section{Liste der Veröffentlichungen}

\section{Wissenschaftliche Veröffentlichungen}

- Sebastian Dreyer: Mikrostruktur und Transporteigenschaften von Ni/NiO - Vielfachschichten. Diplomarbeit, Institut für Materialphysik der Georg-August Universität Göttingen, (2001)

- S. Dreyer, J. Norpoth, C. Jooss, S. Sievers, U. Siegner, V. Neu, T. H. Johansen: Quantitative imaging of stray fields and magnetization distributions in hard magnetic element arrays, J. Appl. Phys. 101, 083905 (2007)

\section{Weitere Veröffentlichungen}

- S. Sievers, M. Albrecht, U. Siegner, S. Dreyer, Ch. Jooss: Combined optical and force microscopy of patterned magnetic films. J. Appl. Phys. 99, 08S504 (2006)

- S. Schnittger, S. Dreyer, Ch. Jooss, S. Sievers, U. Siegner: Magnetostatic interactions in patterned CoPt films embedded in a permalloy matrix. Appl. Phys. Lett. 90, 042506 (2007)

- J. Norpoth, S. Dreyer, C. Jooss: Manipulating the dipolar magnetic interactions in FePt square arrays: The role of edge roughness. J. Appl. Phys. 101, 09F518 (2007)

- C. Herweg, S. Dreyer, P. Troche, J. Hoffmann, S. Sievers, C. Lang, H.C. Freyhardt: Nanostructuring of multilayers by a thermally driven self assembling process. Mat. Res. Soc. Symp. Proc. 704, 161 (2002)

- E. Peiner, M. Oehler, A.C. Mofor, H. ElShaer, A. Waag, S. Dreyer, C. Jooss, L. Doering: Piezoresistive Cantilever Magnetic Field Sensor. Sensor 2005 12th Int. Conf. Proc. 1(1), 213-218 (2005) 


\section{Danksagung}

Für mich ist es nicht allein die sehr gute wissenschaftliche Betreuung, die zu dem Gelingen dieser Arbeit geführt hat, sondern vor allem auch der starke persönliche Rückhalt meiner Eltern und von Stephanie. Besonders in schweren Zeiten habt Ihr mir die Kraft gegeben weiterzumachen. Euch gilt deshalb mein erster Dank.

Herrn PD Dr. Ch. Jooß gilt mein Dank nicht nur dafür, dass er mir die Möglichkeit des Neubeginns der Doktorarbeit eröffnet hat, sondern auch für seine hervorragende Betreuung und sein persönliches Engagement in der wissenschaftlichen Diskussion. Besonders durch sein privates Interesse an einer harmonischen Zusammenarbeit herrschte stets eine angenehme und freundschaftliche Arbeitsatmosphäre.

Frau Dr. Sibylle Sievers danke ich für die tolle Zusammenarbeit und viele schöne MFMMessungen. Ihrer Unterstützung und Koordination ist es zu verdanken, dass zwischen der Arbeitsgruppe Jooß und der PTB Braunschweig eine hervorragende Kolaboration entstanden ist. Desweiteren gilt an der PTB mein Dank Sascha Droste für hochwertige MFM-Messungen und Dr. Thomas Weimann für die ausgezeichnete lithographische Präparation der kleinen Strukturen.

Herrn Dr. Jörg Hoffmann gilt besonderer Dank, da er mir auf meinem langen Weg immer mit Rat und Tat beiseite stand. Nicht nur in wissenschaftlicher, sondern auch in menschlicher Hinsicht konnte ich immer wieder von seinem reichhaltigen Erfahrungsschatz profititeren.

Herrn Dr. Volker Neu und Herrn Patra gilt mein Dank für die Herstellung der Pr-Co-Filme und für viele hilfreiche Tipps.

Bei Julia, Carolina, Jonas, Sven und Peter bedanke ich mich für eine schöne Zeit und gute Zusammenarbeit. Besonderer Dank gilt Jonas für seine unermüdliche programmiertechnische Unterstützung.

Herrn Prof. Dr. H. U. Krebs und seiner Arbeitsgruppe danke ich für eine tolle Arbeitsatmosphäre auf dem 2. Stock und viele schöne Erinnerungen an die Doktorzeit.

Herrn Dr. Kevin Brinkmann danke ich besonders für die Hilfe im Umgang mit Linux und bei der Programmierung diverser nützlicher Skripte und Programme.

Ich danke weiterhin allen Mitgliedern des Instituts für Materialphysik, insbesondere den Mitgliedern der elektronischen und feinmechanischen Werkstatt, die mir bei allen technischen Fragen immer hilfreich zur Seite standen. Herausheben möchte ich Michael Malchow, der bei Problemen aller Art immer eine gute Lösung parat hatte und Karin Ahlborn, die immer ein offenes Ohr für mich hatte.

Zu guter Letzt danke ich allen Freunden, die ich in Göttingen kennengelernt habe und die aus der Studienzeit eine tolle Zeit haben werden lassen. 


\section{Lebenslauf}

Am 30. Januar 1975 wurde ich, Sebastian Dreyer, als Sohn von Hildegard und Friedrich Dreyer in Hannover geboren. Meine Staatsangehörigtkeit ist deutsch. Von 1981 bis 1985 besuchte ich die Grundschule in Wennigsen (Deister). Im Anschluss daran absolvierte ich von 1985 bis 1987 die Orientierungsstufe in Wennigsen und ging im Jahr 1987 zum Matthias-Claudius-Gymnasium in Gehrden. Dort erwarb ich 1994 meine allgemeine Hochschulreife.

Nachdem ich meine Wehrdienstzeit im Transportbataillon in Dörverden geleistet hatte, begann ich im Oktober 1995 das Studium der Physik an der Georg-August-Universität Göttingen. Die Vordiplomsprüfung erfolgte im Frühjahr 1998. Ende 1999 begann ich mit der Anfertigung meiner Diplomarbeit mit dem Titel Mikrostruktur und Transporteigenschaften von Ni/NiO-Vielfachschichten unter der Leitung von Prof. Dr. H. C. Freyhardt am Institut für Materialphysik. Im Mai 2001 legte ich die Diplomprüfung ab.

Von August 2001 bis September 2003 arbeitete ich als wissenschaftlicher Mitarbeiter in der Arbeitsgruppe Freyhardt und wechselte im Dezember 2003 in die Arbeitsgruppe unter Leitung von Priv. Doz. Dr. Ch. Jooß am Institut für Materialphysik. Dort entstand die vorliegende Arbeit. 